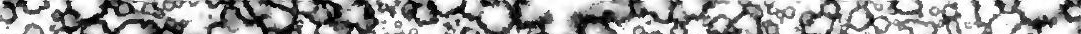

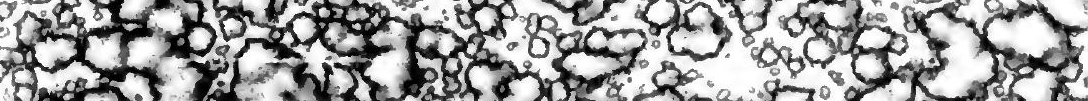

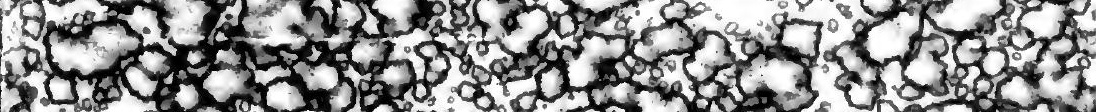

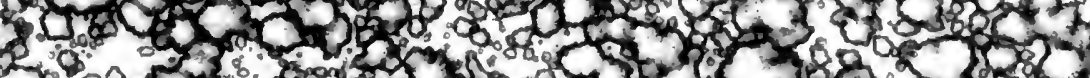

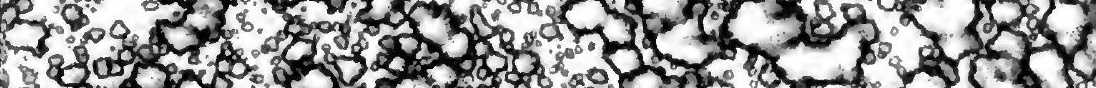

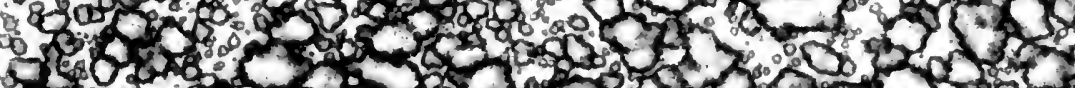

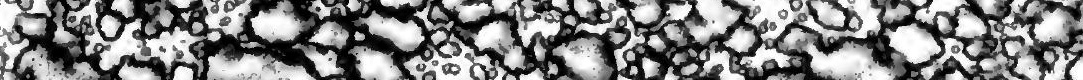

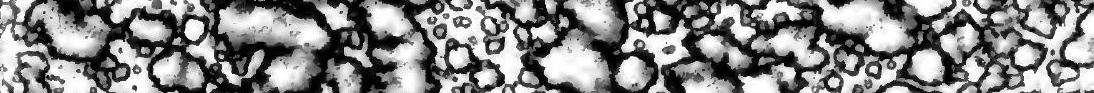

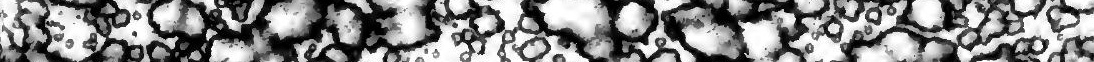

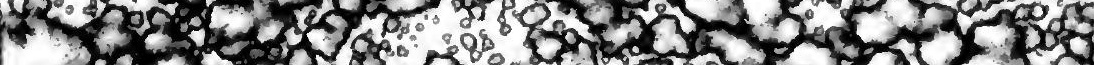

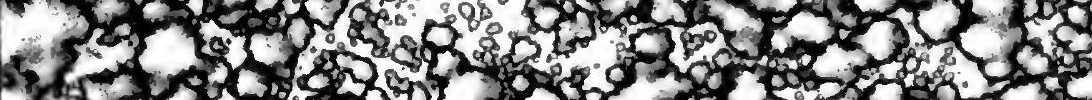

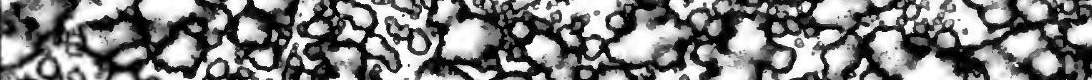

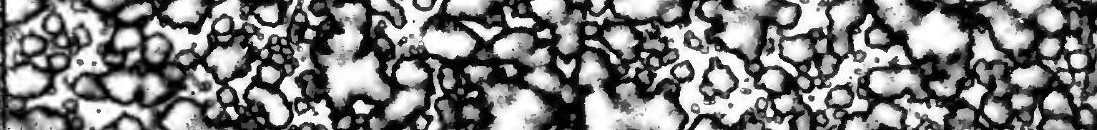

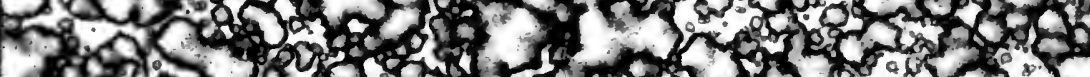

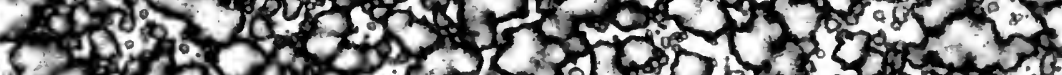

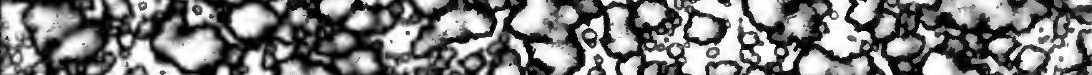

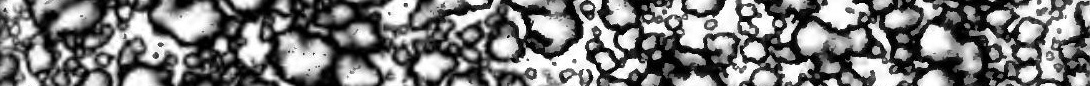

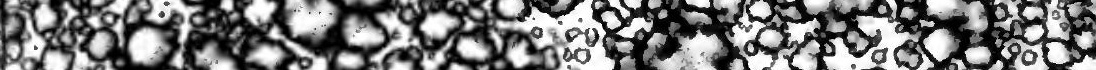

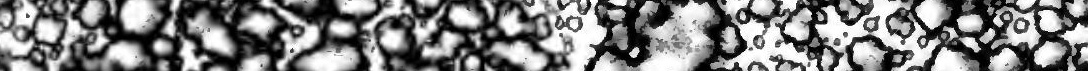

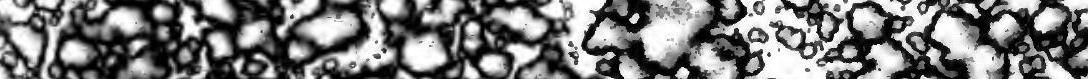

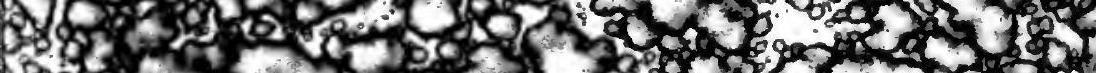

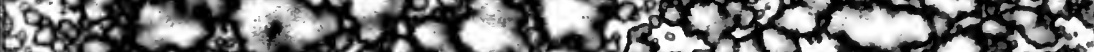

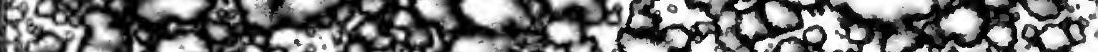

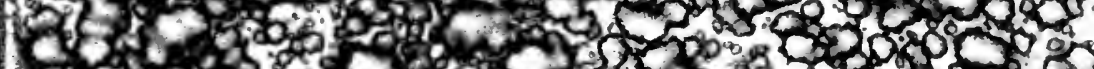

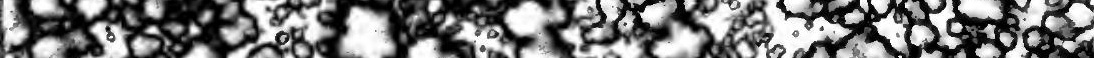

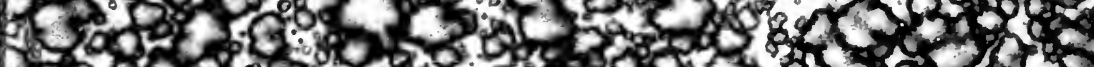
20 -2 $5805 \%$.

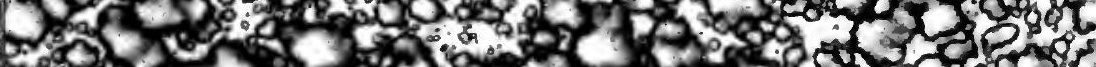

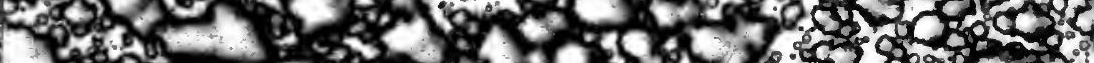

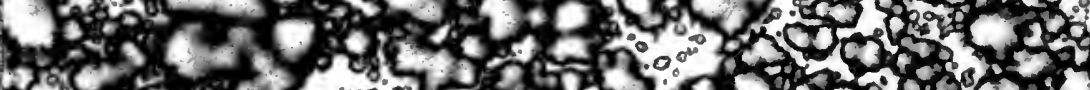

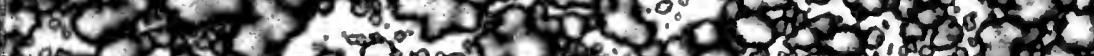

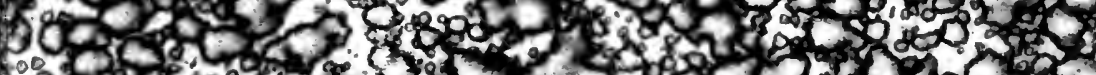
100 क

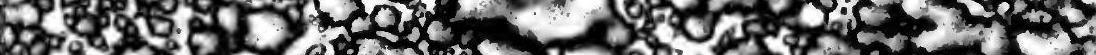

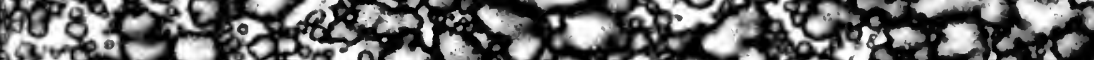
S.

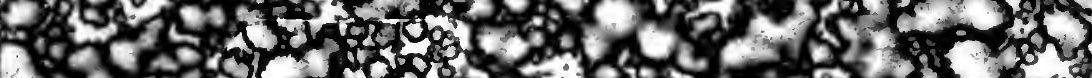

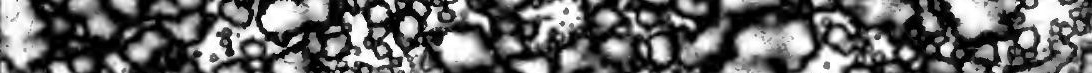

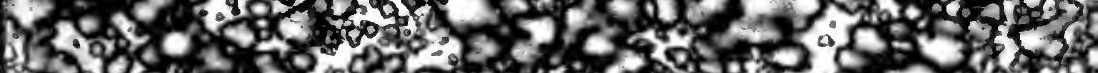

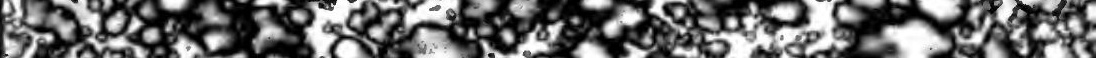
(5)

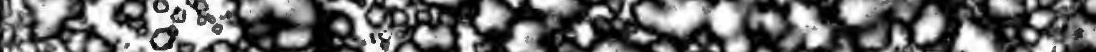

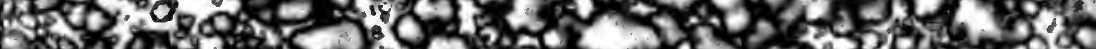

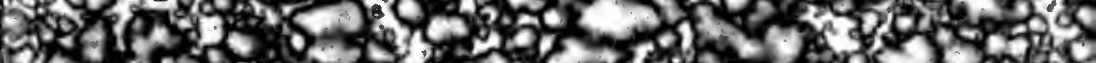

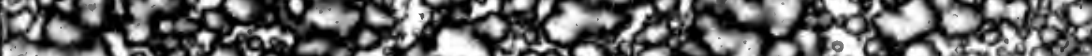

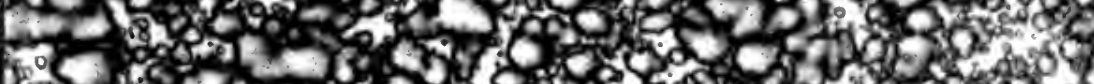

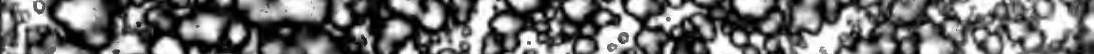

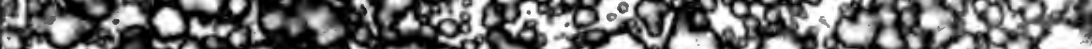

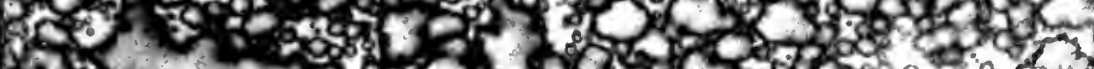

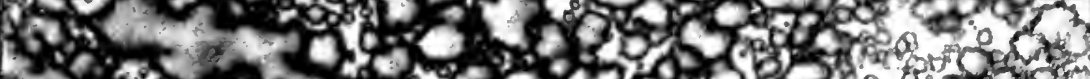

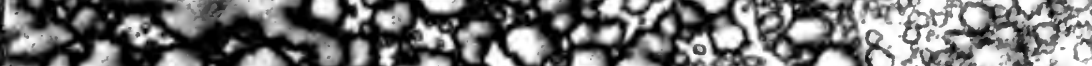

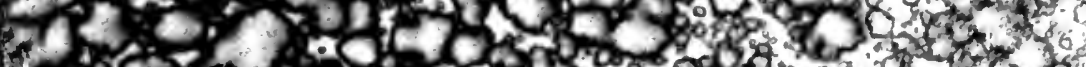


is

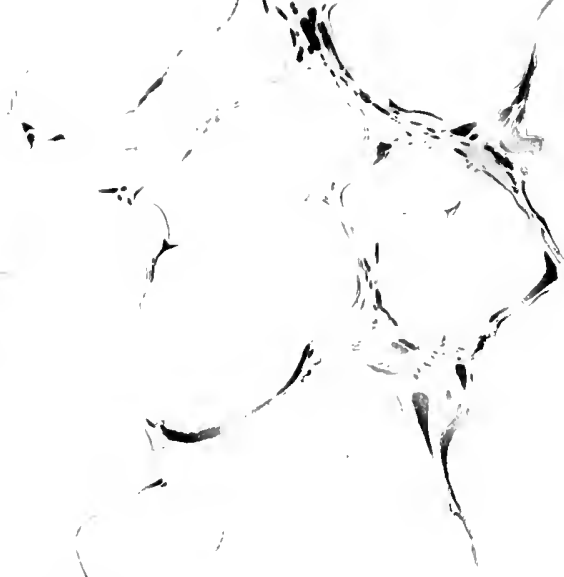

i

,

i

तो
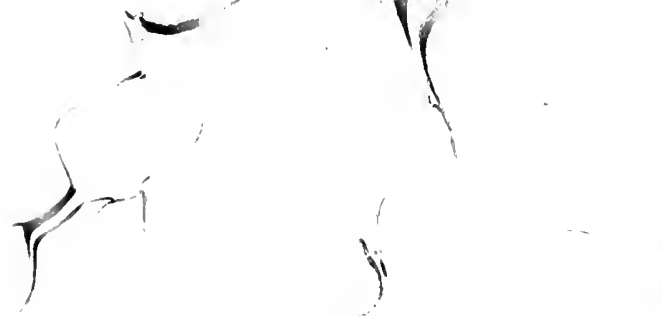


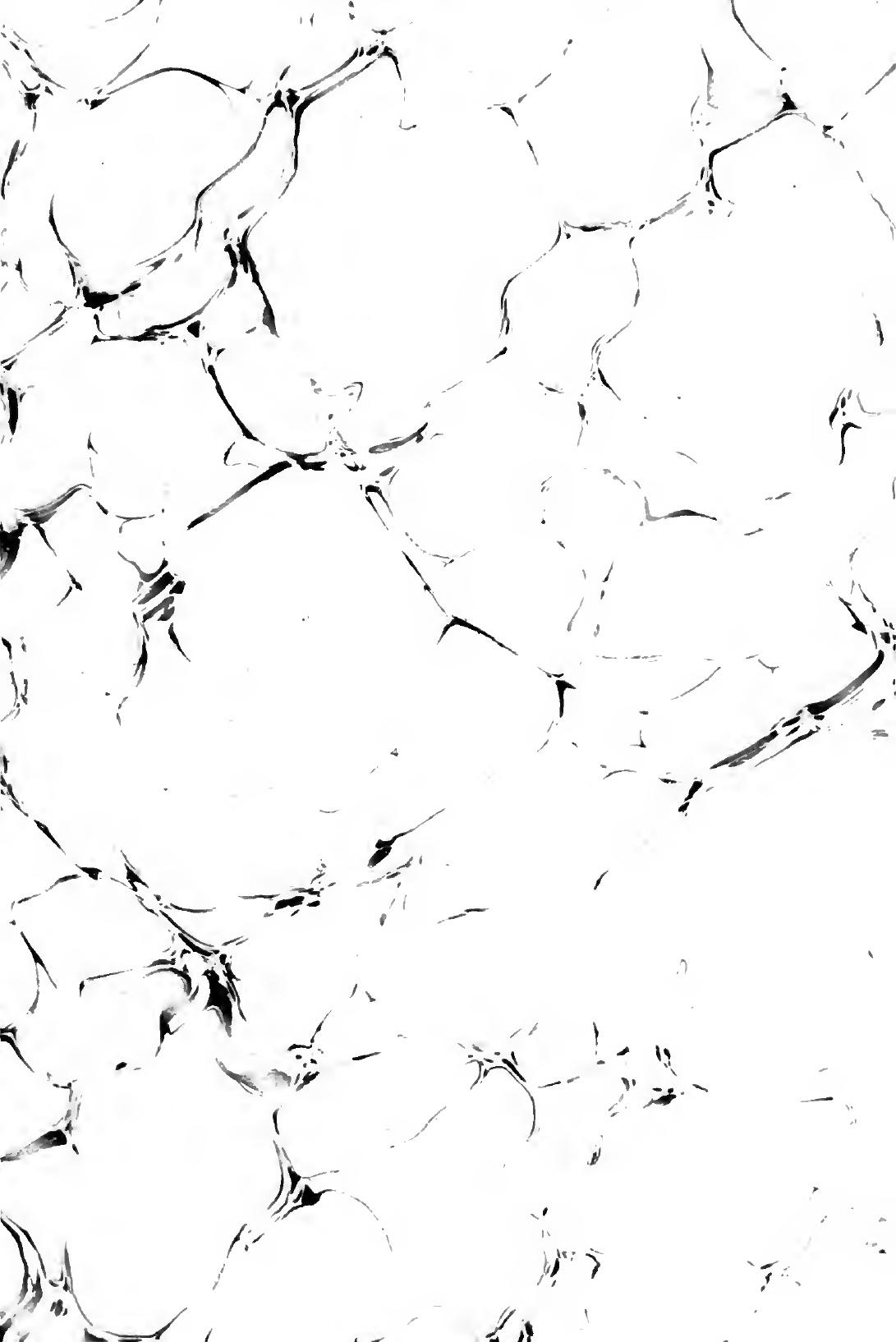









\section{E}

MOUVEMENT DE CONCENTRATION

DANS LES

BANQUES ALLEMANDES 



\title{
EDGARD DEPJTRE
}

\author{
Docteur en Droit
}

\section{工E}

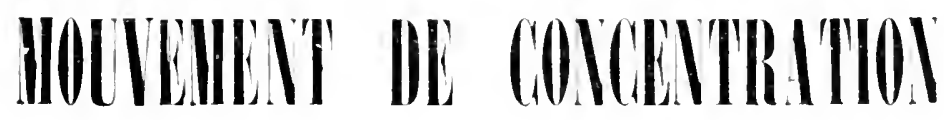

1). NS 1.R

\author{
BANQLES ALLEMA $\backslash D E S$
}

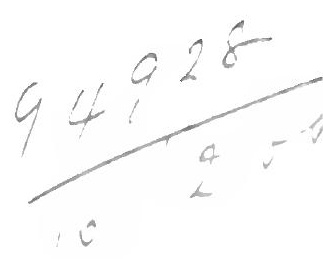

\section{PARIS}

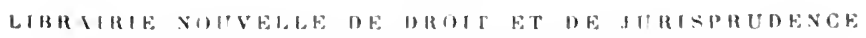

ARTHIR ROLSAEAL, EUTTEU

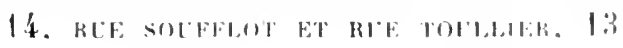

1905 



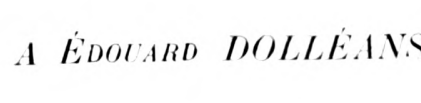





\section{INTRODUCTION}

L'évolution industrielle et commerciale moderne pousse a une concentration toujours eroissante des capitaux. "liest là un fait si universeltement connu, si fortement établi par des observations nombreuses at comcordantes qu'il ast devenu banal de le constater (1)." Le nombre des entreprises diminue a mesure que s’aceroit l’importance de celles qui survirent: la seconde moitié du xix siecte a été caractérisée par le recul prespue général de la petite exploitation, du málire, du petit commerce, - par l'arinement de la grande industrie, des sociétés par actions, des syndicats de sociétés, - par la création de moyens de production de phus en plus pruissants, d'orgunismes cominericiane de plus an plus considérables.

Le commerce de banque n'est point resté en dehor's de ce mouvement général. Schathle a montré comment dans la banque plus encore que lans toute autre industrie, la centralisation était particulierement livorise alu point de vue technique et nécessité au point de rue économique (2). Aussi bien des hats nombreux et précis sont-ils renus élablir que la tendance da monde moderne de lat

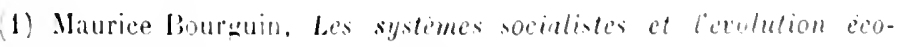
nomque, p. 13i.

3) Schaeffle, Das yesellschufluche System der menschlichen IIntschatt, II, p. 15i. 
banque tendait deplusen plas vers la concentration : la rapide agótomération des petiles banquesest, uon moins que la concentration des entreprises industriclles, l'une des évolutions les plus frappantes de ce dernier quart de siècle en Europe et aux Etats-Unis (1).

En France nous arons assisté ì cette raréfaction des banques locales devant l'action combinée et progressive des trois grandes sociétés de crédit: Crédit Lyonnais, Comptoir National d'Escomple. Société Générale. Ces trois établissements représentent aujourd'hui un capital-actions de 600 millions de franes, plus 140 millions de réserves et possèdent tant en France quà l'étranger plus de 900 succursales ou agences. - Nous aurons à esquisser maintes comparaisons entre la politique suivie par les grandes sociétés françaises et celle quont préférue les grands établissements berlinois, maints rapprochements entre les résultats obtenus de part et d'autre. Pour l'instant, nous nous bornons à rappeler ce fait de la concentration dans les banques françaises, fait qui déjì a été longuement étudié dans la thèse, à lous points de vue remarquable, de M. Maurice Saurel (2).

En Angleterre et daus le pays de Galles où l'on comptait en 186't - en dehors de la Banque d'Angleterre et de ses succursales, - 278 banques privées et 117 Joint-StockBanks, l'on ne retronvait plus en 1900 que 53 banques privées et 83 Joint-Stock-Banks, en 1905,62 Joint-Stock-

(1) A. C. Conant, "The Evolution of moderne Banking " (Political Science Quaterly, décembre 1854, 1. 569).

(2) Minurice Saurel, Societén de credil contre banques locales. Thèse Paris, 1901. 
Banks. Le lableau suivant montrera la diminution progere sive du nombre de ces dernières (1):

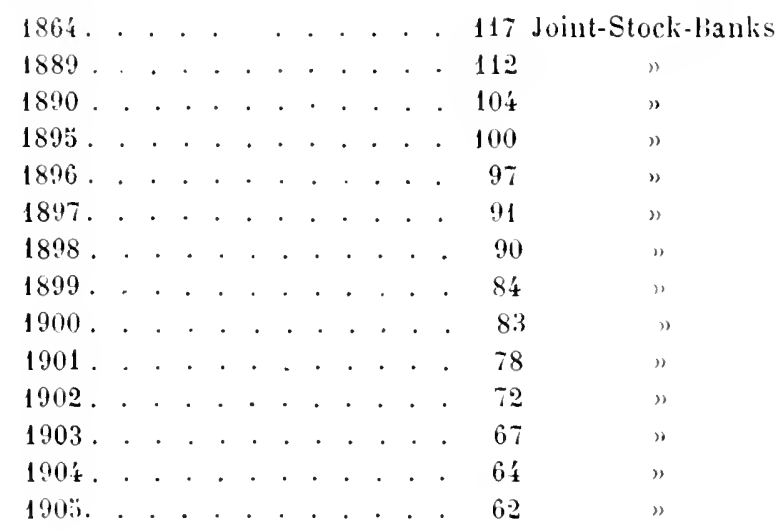

Le mourement s'est donc précipité en ces toutes dernières années. Nous sommes amenés à faire la mème remarque, si nous considérons maintenant les banques privees (2):

\begin{tabular}{|c|c|c|c|}
\hline 1837 & 448 banques privées & 1891 & 1'i banques privées \\
\hline 1858 & 261 & $1896^{\circ}$ & 100 \\
\hline 1878 & 220 & 1900 & 53 \\
\hline
\end{tabular}

Le London Bankers Magasine de février 1899 releviit de 1877 a 1886. 42 fusions de banque; de 1887 a 1898 , 12 fusions de banque; on comple, de 1899 a 190̈̈, S6 fusions de banques.

A Londres senlement le nombre des banques - it l'exception des banques coloniales - a passéde 11 i en $188^{\circ}$, ¿̀ 77 en 1901, à 38 en $190 \%$.

En 1901, l'on comptail plus de 20 banques possédant chacune an moins 100 sucrursales: en 1904 . on morompte

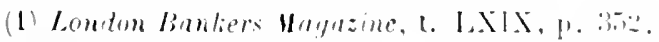

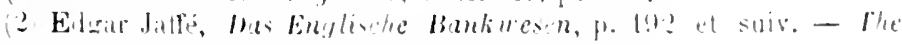
Economist-Bunting sinplement, 21 octobre 1905. 
pas moins de 4.417 succursales de banques, 4.458 en octobre 190\%.

Inversement, le capital des entreprises survivantes n'a cessé d'augmenter: lo total du capital versé des JointStock-Banks du Royaume-Uni représentait:

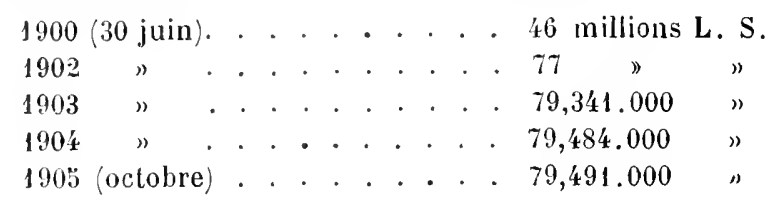

"Je ne puis prévoir avec certitude, écrivait déjà Bagehot en 1873, la continuation de notre système de banques particulières: jai la conviction que les jours des petites banques sont comptés et qu’elles disparaîtront avant que bien des années soient éconlées. Quant aux grandes banques parliculières elles ont à surmonter de formidables obstacles...." (1) et, de fait, "pour celui qui examine les changements qui se sont produits dans les banques anglaises depuis un quart de siècle, la caractéristique du mouvement est, avec une augmentation progressive des ressources, la tendance toujours accentuée à la centralisation des forces financières par la fusion, l'amalgamation des banques de Londres et de province " (2).

Aux Etats-Unis enfin, où la législation a imposé aux banques les conditions particulières, nous trouvons encore peu de grandes hanques aux nombreuses filiales: mais la formation des trusts n’en a pas moins accéléré le mouvement de concentralion dans les affaires de banque, en privant les banques locales d'une partie de leur clien-

(1) W. Bagehol, Lombard Street, ou e marchi financier en Angleterie, 1. 26 :

(2) G. François, "Les banques anglaises", Revue d'économie politique, juillet 1902. 
tèle industrielle el en augmentant l'importance des griandes banques de New-York qui participent aux principaux trusts.

Une large part des réserves des banques des petites et moyennes localités s'est concentrée lans les grands centres: e'est New-lork surtout qui a profité de cel afllux et de plus en plus les réserves saceumulent dans la capitale comme en Angletere on an France. An 1:̈ septembre 1902, les 43 hanques nationales de Nen-lork ne possídaient pas moins de fli millions de dollars de dépoits d'autres banques (1) : ou ces cinq derniòres années, te capital des banques nationales de New-York a passe de 232 à $40 \% 1$ millions de dollars.

En 1898, deux hanques senlement possédaient un eapital de $\$ 00.000$ dollars : en 1901 trois hanques avaient un capital de 10.000 .000 de dollars: une, la Yalionale Lity Bank, un capital de 2:3000.000 dre dollar's; en 190', on compte 8 hamques d'un capital d'au moins 10.000 .000 de dollais.

Ces augmentations du capital-alctions sont dues en grande partic à des lusions. Lal Nalional bank of Commerce, fusiomie avee la Western National Bank, dispose

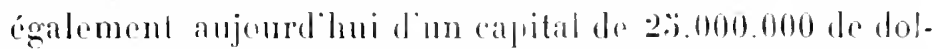
lars. En 1901, 27 banques natiomales, en 1902, th lanques nationales et 11 autres banques. en 1903-1901, 3s banques ont été ahsorbées.

La concentralion a pris anx Etats-Inis une forme originale et qui présento haiucoup d'analogies avee ces groupements de banques que nous allons retrouver en Allemagne. Les Antíricains appellent ces féderalions "des

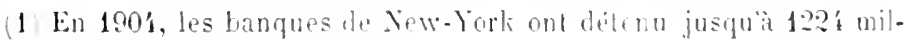
lions de dollars de deiputs. 
chaînes de banques" : elles se sont a vant tout constituées sous l'influence personnelle des grands fondateurs de trusts. C'est ainsi que nous nous trouvons en présence :

$1^{\circ}$ Du groupe Rockfeller, qui dispose d'un capital de 108 millions de dollars; il a à sa tète la National City Bank;

2. Du groupe J. P. Morgan, dirigé par la First National Bank (capital 97 millions de dollars);

$3^{\circ}$ Du groupe Morse (23 millions de dollars) et de la National Park Bank (13 millions de dollars) contròlé par les Astor, les Vandrorbilt et les Belmont.

Les Banques d'une même chaine ont des intérèts communs et les intérêts des chaînes de banques s'entremêlent, de sorte que ces groupes sont régis par une politique commune. Ce mouvement de concentration dans les banques des Etats-Unis est dans un rapport étroit avec le mouvement de concentration industrielle et commerciale et les grands trusts.

Deux traits principaux caractérisent les grands établissements conducteurs du mouvement de concentration. leur puissance de capital d'une part, leur diffusion de l'autre; ce dernier caractère surtout, particulièrement propre à la grande banque, la diflérencie nettement de la grande industrie an double point de vue et des causes de sa supériorité technique at déla forme que va prendre le processus de concentration.

"La cause générale du mouvement de concentration est bien connue, dit encore M. Bourguin ; c'est la supériorité des grandes entreprises dans la concurrence qui le provo- 
que avec la forre irrésistible d'une loi naturelle 1). ".ais

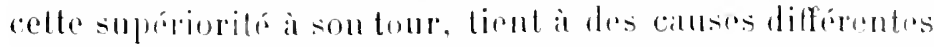
et les arantatges de la concentralion ne sont point les mèmes, suivant quion considere lindustriroule commerce de banque.

Dans l'industrie, la supériorití de la garande entreprise consiste principalement en ce fait qu'elle peut produce a meilleur compte : cul abaisoment du cout de production résulte de res trois causes assenticlles et birn connues:

Perfectionnement de l'ontiltage;

Systime plus complet de division du travail;

Economic de frais sénéraux.

Or, la suprériorite de loutillage. essenticlle dans une entreprise industrielle, na dans la hanque qu une importance secondaire, pour cette simple raison, dit II. Silurel, quine machine ne saurait aceomplip qu'une tiche matérielle et que le travail matéricl d’une banque n’existe pour ainsi dire pas (2). Il laut cependant se garder d'exagérer: le travail matériel loune bingue ast loin d'otre nul et il peut sexécuter dans de gramb ritahlissemenis bien outillés avec unc simplicité et mo rapidité plus grandes (vastres localux bion aménigés, imprimés pour affares courantes, service particulier do postes, reception et axpidition, ote... Bagehot qui as-i-tait aux premieres transformations des banques priveres en sociétís par actions et dent les observalions nous sont d'ume prócieuse ntitite. parle qualque part de "detail incroyable " que comporte lat banyue moderne; "il fant. dit.il, mn

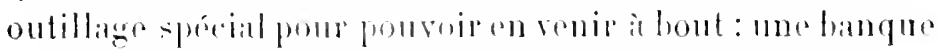
par actions bien montée posside cet ontillagen "3).

(1) Bourguin, op. cit., p. 1'?

(2) Saurel, op. cit., p. 1?.

(3) V. Bagehot, op. cit., p. :033. 
De même en ce qui concerne la division du travail. Si l'on excepte certains services (contentieux, études financières...) qu’on ne trouve point nettement spécialisés dans une maison même de moyenne importance, on peut dire que la division du travail n'est guère plus complète dans les grandes que dans les petites banques, - ce qui tient au travail même do banquier et à la diffusion des grandes banques.

La supériorité de ces dernières ne paraît point venir davantage de la modicité relative de leurs frais. Au contraire, des obligations de trésorerie plus considérables, de plus lourdes charges fiscales, les dépenses d'installation d'un grand nombre de succursales et agences, le luxe des sièges principaux, la rémunération d'un personnel pro. portionnellement plus nombreux, les frais d'un vaste et minutieux contrôle ont semblé, à quelques-uns, venir ici au désavantage des grands établissements (1).

Cela nous paraît surtout vrai d'ailleurs des grandes sociétés françaises de crédit qui n’ont guère pratiqué que la politique d'extension directe par agences et succursales. Nous verrons, all contraire, qu'en Allemagne, les grands établissements ne possèdent qu'un nombre relativemont limité de filiales proprement dites dans les grands centres commerciaux. Comme la plupart des banques anglaises, les grandes banques berlinoises ont pratiqué de préférence la politique "d'amalgamation" ou de fusion, ce qui rend possible une certaine réduction des frais d'exploitation, "sinon toujours immédiate, au moins éventuelle" (2). 1) est très rire, par exemple, que tous les directeurs des

(1) V. Saurel, op. cit., p. 19-26.

(2) K-J. Steele, "Bank Amalgamations " (Economic Journal, décembre 1896). 
deux banques fusionnées fassent partie du nouveau conseil d'administration: Lors de la fusion de la Consolidated et de la l'arr's Bank en 1896, six senlement sur treize des directeurs de la Consolidated prirent place dans la nouvelle direction: lors de la commandite de la maison $R$. Varschauer de Berlin par la Darmstïdter bank en 1898, le troisième associé de la maison Varschauer se retira.

Dans les villes de moyenne importance, les cablissements berlinois se contentent de simples correspondants; ils s’allient aux banques déja existantes ol conduisent leurs affaires de conecrt arec elles : un tel procédé n’exigne point de grosses dépenses d’installation. il nécessite nn contrôle moins strict, les charges fiscales ne sont point augmentées. Ưne certaine beonomie des frais généraux peut done trouver place au nombre des avantages de la grame banque ainsi comprise, mais sans toutefois jouer le mime ròle que dans la granile industrie.

Quelles sont done les canses de la suprériorité tachnique des grandes bancues? Ia fonction distinctive du banquior, dit Ricardo, commence des qu’il emploiel'arsent des antres : le ròle le la banque se résume en ees deux mots: emprunter pour priter. Les banques sont des intermidiaires de crédit: "par les dépòts, les négnciants font erólit au banquier, et d'autre part redui-ci en probant sons des conditions détermináes les fonds déposiss a qüil peul faire puisqüil nest pas tenu de reslituer les fomls mimes qu’il a reçus, fait crédit aux industriols qui ont hesoin d'argent” (1). La supériorití les grandes banques ral rósider ence fait quelles emprunteront el quelles priteronl plus

(1) Cauwès, Cours d'économie politique, II, p. 278. 
facilement : elles offrent une meilleure adaptation au mécanisme de l’offre et de la demande. Ceci se résume en ces trois aspects :

a) Tout d'abord, le grand établissement est assuré d'une plus large compensation des risques par l'effet bien connu de la loi des grands nombres. Quand les opérations d'une banque atteignent un chiffre considérable, les probabilités de pertes ne s'écartent guère d'une certaine moyenne et cette stabilité des résultats est infiniment avantageuse: le risque sc réalisàt-il, il est incomparablement moins grave : "la perte se trouve noyée dans une telle masse de résultals que l'effet en est considérablement amorti : dans un faible cours d'eau, les moindres aceidents climatériques produisent des oscillations de niveau considérables; mais le fleuve majestueux en roule des flots à peine cliangés» (1).

Aussi bien " est-ce un fait curieux et bien connu, que l'appréciation du risque est plis exacte quand on l'envisage avec un certain recul " que va permettre le contrôle superposé des agences ou des banques correspondantes et du siege central. Et ces considérations prennent à leur tour une plus grande importance, quand il ne s'agit plus seulement de banques ordinaires, se livrant aux seules opérations courantes de banque (legitimate banking, reguläre geschäft), mais birn quand nous nous trouvons en présence d'institutions qui, comme les banques allemandes, sont autant des Sociétés financières que des Sociétés de crédit. Nous verrons à quel point les lanques allemandes sont intéressécs directement dans les entreprises industrielles et commerciales : felle graude banque aux nomhreuses participations poura supporter sans faiblir une

(1) Saurel, op. cit., p, 36. 
perte même considérable, assurée quelle est d'auter part de revenns b́levés, - témoins les importints capitanx con. fonis par la Discontogesollschaft dans les chemins de fer vénézuéliens on dans la Compagnie Popp. Si la banque an contraire n'a fait profiter de son crédit et de ses capitaux qu'une seule entreprise, la ruine de cette derniere entraînera presque fatalement sil propre chute et l'on pent citer ici lexemple - toutes autres considérations mises à part - des opérations malleureuses de la Leipziger bank arec la Treber Tröcknung de Cassed.

b) En second lieu, le grand établissement va pouvoir exercer une action plus étendue sur le public: par la multipliciti de ses agences il conquiert une immense clientile, par ses succursales ou ses correppondants il rayoune sur tout le territoire. Les mallodes utili-ís prour assurer cette diffusion d'atetion pourront varies et nous verrons comment en Allemagne elles different sensib]ement de celles qui furent employées en Angleterre ou en France: le rósultat cherché - el obtenu - reste le mème: assurer en quolque sorte l'ubiquite de l'atablissement, lui permettre de multiplier les affaires. ... et par lia mime les prolits, - Le meltre a mime par ces multiples canaux, agences et liliales, de pomper partout tes capitaux thottints pour les refouler ensule sur lons les points oil ils sont nicessaires; - commo ans hi hen venir fortifier chatque agenere on chaque filialde proprede toute la force de chacun des rouages de l'omorme mathine: "Nos retalions

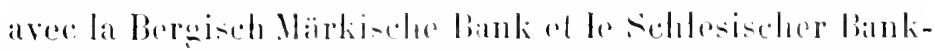

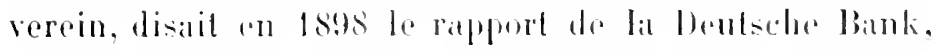

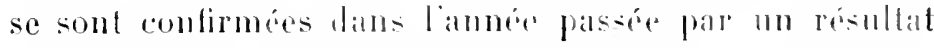
aussi favorable pour ane mations que forme nous-mimas.

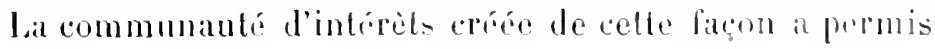


d'assurer à la clientèle de ces établissements de grands avantages, grace à l'amélioration des informations, à une facilité plus grande de combiner leurs affaires, quils ont trouvées dans laide complaisante de plusieurs directions de banques maintenant à leur disposition..."

La puissance de crédit attachée à la signature des grands établissoments, la publication de leurs bilans "qui leur sert d’annonce perpétuelle "(1) complètent cette action en profondeur, pour ainsi dire. - " On choisit naturellement pour banquier celui qui a le plus de crédit au moment présent, dit Bagehot. - Or c'est le banquier qui a le plus d'argent entre les mains qui a le plus de crédit " (2). Et s'il était besoin de faits pour éclairer des vérités si évidentes, il suffirait de rappeler comment en Allemagne, ce fut au lendemain des crises économiques de 1873 , de 1891 et de 1901 que le public se détacha des banquiers privés ou des petites banques, pour se rapprocher des grands établissements berlinois qui lui offraient de plus sérieuses garanties apparentes.

c) La supériorité du grand établissement se manifeste enfin dans ce fait qu'entre ses mains les capitaux ne resteront jamais incmployés et improductifs; "établi sur un graud nombre de places oì les conditions économiques sont très diflérentes, il ressentira directement toutes les oscillations du marché de l'argent et pourra y répondre aussitôt "2). Aussi bicn son activité embrasse-t-elle un large consemble d'opérations ; il portera, par le simple jeu de l'organisme, ses capilaux dans la branche d'affaires où ils sont le plus demandés, cest-i-dire le plus nécessaires et le plus lucratifs. L'actisité d’une banque locale reste

(1) Bagehot, op. cil., p. 257.

(2) Saurel, op. cit., p. 90. 
forcément enfermée dans les limites d'une place déterminée. La diffusion seule de l'établissement peut assurer cette mobilité et cette fructification constantes des ressources.

Ainsi, ce sont les conditions techniques elles-mèmes qui ont commandé et qui nous expliquent la différence des formes que revit la concentration dans l’industrie et dans le commerce de banque.

Les avantages de la concentration paraissent dans l'industrie altachés à la dimension de l'établissement; dans la banque ils le sont plutỏt à la dimension de l'entreprise. "La concentration industrielle aboutit is la constitution d'un établissement énorme et unique, englobant sur un seul point tous les rouages qui doivent concourir à la production. "La concentration financière se manifeste, elle, par multiplication des établissements, agences, succursales ou correspondants, plutòt que par agrandissement d'un siège unique: elle val réunir sous une mème direction un grand nombre d'établissements, ayant ehacun leur vie propre, comprenant chacun les parties d'un tout complet, mais aussi recevant tous une mìme impulsion et concourant au mème but (1).

Tels sont rapidement esquissés et le lait général de la concentration daus le commerce de banque, et sa cause première, et sil forme accoutuméc. Mais ce ne peut ìtre lì qu'une esquisse toute théorique et générale. Le mouvement de concentration dans la banque est loin d'avoir suivi, dans tous les pays, une marche uniforme el paral-

(1) V. Bourguin, op. cit., p. 1it; Saurel, op. cit., p. 13. 
lèle: des caures secondaires, juridiques, financières, politiques mème, sont venues, soit retarder, soit au contraire précipiter cette tendance: elle a, suivant le génic de chaque peuple, affecté des modalités particulières et les moyens employés diffèrent sensiblement.

Si, par exemple, l'un des principaux titres d'une telle recherche est l'intérèt qui provient de l'interdépendance reconnue de la banque et de tontes les autres manifestations de l'activité sociale, - interdépendance qui donne au développement de la banque un intérêt général et nous permet d'y voir comme un reflet, ou mieux comme une synthèse des tendances qui agissent dans toutes les autres branches de production, - on comprendra aisément l'importance que va présenter la fiçon mème dont tel ou tel peuple comprendra et pratiquera le commerce de banque. Ici, les banques se montreront timides el peu empressées ì soutenir industriels ou négociants, - ou bien les fonctions de crédit nobilicr seront nettement différenciées des fonctions de dépôt et d'escompte: là, au contraire, nous trouverons des banques dont l'activité plus complète et plus lardie embrassera tout le domaine économique et qui, comme le dit Scharling, seront dans ce pays, à la fois "les promoteurs et les bénéficiaires du développement industriel et commercial ". Ces deux conceptions différentes vont agir dans un sens contraire. l'une favorisant, précipitant, l'autre contrariant le développement de la banque et par là même le mouvement de concentration.

On peut aussi entrevoir d’autres corréquences, agissant dans de mèmes directions: ces rapports plus ou moing étroils des ćtablissements do crédit avec le commerce ou l'industrie nationale détermineront le caractère plus ou moins spéculatil des banques: elles souffriront donc plus 
ou moins des makaises at des crises f́conomiques gue traverserale patys: aes crisses ne rout point non plus sans atlirer l'attention des pouvoirs publics et laction législative à son tour, dont les conséfuences directes on indirectes, ne sont point toujours celles qu'avaient prévues les législateurs, pourra déterminer des courants non négligeables.

Montrer pour le commerce de banque en Allemagne, quelle fut la mitrehe progressive du mouvement de concentration en ees dernières années, exposer les résultats momentanément alequis, délimiter la part des causes particulières qui vinrent soutenir la tendance générale à la concentration ef forlifier la supériorilé des grands établissements, examiner enfin, lans quelle mesure, les moyens techniques utilisés en Allemagne se sont éloignés du type classique, peut-on dire, de concentration dans la Banque et dont l'Angleterre ou la France nous donnent un exemple assez net, - tel ast le but que nous nous sommes proposé.

Lne telle reclserche présente deux aspects: on peut considérer le mouvement de concentration ì un point de vue extrme, c'est-ì-dire étudier l'évolution dans son ensemble, en exposer lis causes et les modalités, grouper les résultals généraux : on peut aussi à un point le vue interne, reprendre le diveloppement historique de chacun des grands établissements survivints et prépondérants, et successivement exposer son róle particulier dans le processus de concentration. le résultat particulier auquel il est parrenu.

Nous arons cru, afin te demner une plas exacte physionomie du tablean de la concentration dans le banques 
allemandes, devoir l'exposer' sous chacun de ces deux aspects, l'un synthétique, l'autre analytique. Ils correspondent à chacune des deux parties qui forment l'ensemble de cette étude.

D'autre part, nous avons limité notre recherche aux banques d'effets proprement dites (Еғfектелвanken), c'està-dire à ces banques qui, suivant l'expression de Wagner, sont les véritables conducteurs du mouvement moderne de la banque. Elles correspondent à ce qu'en France nous nommons les établissements de crédit, réunissant les fonctions de dépôt et d'escompte; mais elles remplissent également des fonctions de crédit mobilier et présentent par là des caractères particuliers que nous examinerons plus en détail.

Nous avons laissé en dehor's de notre étude les Banques hypothécaires: la nature de leurs opérations, - crédit immobilier, - indique d'elle-mème que ces banques sont, dès le début, exploitées sous forme de grandes sociétés par actions : la tendance à la concentration n'y apparait point encore : leur nombre a augmenté au contraire, passant de 29 en 1883 à 40 en 1904 .

Nous n'avons point davantage considéré les Banques d'émission, bien qu'une concentration assez rapide se soit manifestée dans cette catégorie si importante de banques. leur nombre a diminué d'une façon notable: on comptait en 1875, 3马 banques d'émission en Allemagne, 18 en 1883, 6 seulement en 1904.

Doit-on, comme le voudrait Conant, considérer uniquement les banques d'émission comme les apôtres du crédit, "les pionniers de la banque de dépôt "? Leur importance économique est-elle appelée à diminuer de jour en jour au profit des grands établissements de crédit, et ne conser- 
veront-elles plus finalement que le ròle de garliennes dus réserves métalliques du pays? (1) Cest toute une autre étude qu il faudrait faire pour éclaircir ces questions. Aussi bien, croyons-nous, que dans ces résultats que nous venons de rappeler, il faut laire une large part a laction de causes purement artificiclles. La loi d'Empire du 1 't mar's $187 \%$ a voulu préparer l'unité de l'émission fiduciaire et assurer la prédominance de la Reiclısbank, d'oì le nouvelles obligations imposées aux Notenprivathanken, des prescriptions gènantes pour leur activité: nous les royons peu à peu renoncer à un privilège onéreux (2).

Enfin quelle méthode suirrons-nous ? Chercheronsnous, en introduisant dans l’étude des faits un élément subjectif d appréciation, à porter un jugement sur ces phénomines que nous aurons pu conslater? Suivrons-nous

(1) Conant, art. cit.

(2) La mème évolution se constate pour les Banques d'ámission en France où. avant 18's, il existait un certain nombre de bannes lépartementales d’émission $\gamma$ : la Banque de France n’avait le monopole que pour Paris. Des 1810 on ne laissa plus se fonder de nourelles banques départementales: les autres disparurent lor's de la crise de 18:8, absorbées par la Banque de France; celle-ci obtenant le enurs forcé de ses billets on crut nécessaire, afun d'aroir l'uniformite the ha monnaie de papier, de ne laisser subsister quine seule banyue.

En Angleterre, au moment de linte de Peel to's interdisant al'a-

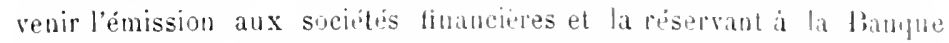

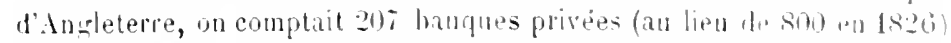

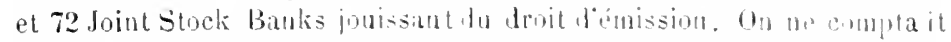

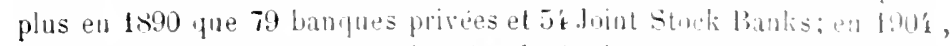

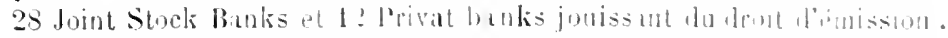

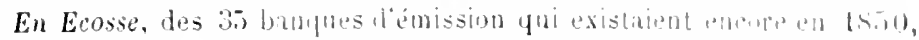

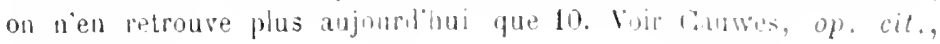
p. $2.25-235$. 
au contraire une méthode purement objective, la méthode réaliste? C'est de cette dernière méthode que nous nous réclamons.

Nous nous sommes efforcé uniquement " de mesurer par des chiffres l'étendue actuelle du mouvement de concentration dans les banques allemandes et surtout la vitesse de son cours "; nous n'avons voulu que préciser des laits, comme aussi bien leur garder leur signification réelle en les maintenant, comme disent précisément les Allemands, dans "leurs conjonctures", dans leur milieu historique.

Fallait-il porler un jugement sur les conséquences de l'évolution? Après avoir exposé ces deux faces parallèles du processus de concentration, - disparition des petites banques, développement progressif des grands établissements, - devions-nous mettre en balance la sonme des avantages et des inconvénients que comporte nécessairement chacun de ces deux systèmes d'exploitation du commerce de banque, nous prononcer en liaveur de l'un d'eux et tirer des conclusions pour l'avenir? Nous ne nous sommes pas cru autorisé à le faire et cela pour plusieurs raisons.

Et tout d'abord, nous dirons que les grands établissements de crédit ne nous appraraissent point, ainsi que semblent les considérer certains auteurs, comme des institutions arbitraires, "monstruosités imaginées par un esprit malfaisant " et semant la ruine autour d'elles, - mais bien plutôt comme des organismes plus vastes et mieux outillés à l'adaptation de l'offre à la demande, nécessités par tout le développement économique moderne. Sur ce sujet particulier trop de pages intéressées ont déjà été écrites, violents réquisitoires contre les grandes banques, 
lamentalions platoniques sur la disparition aes hangues locales, qu un sens plus averti de l'érolution historique, le seul surci de la recherche réaliste, eussent firil éviter. "Tout progrès économique, en ellet, ost suscité par un besoin d'adaptation (1)" ou, comme le dit plus explicitement harl Marx, "de nouvelles conditions de production - et déchange, ajouterons-nous, - ne prennent pas leur place arant que leurs raisons d'ètre matérielles no se soient développées au sein de la vieille Société."

Mais eeci à son tour ne reut nullement dire que les grands établissements soient la forme définitive et immuable que doive prendre l'exploitation du tralic de hanque et que les phénomenes de concentration soient deslinés a se reproduire sans cesse avec une intensité et me importance croissantes. Nous pelisons pouvoir prendre cette expression de K. Marx comme formule génerale d'évolution, sans lui donner un sens doetrinaire. Le capitalisme, en effet, n'est point le seul courant qui traverse les socićtés modernes; à còté de lui, nous trouvons un autre ggrand courant qui vient agir au contraire "dans le sens de la consolidation et de l'intégration par le groupement des éléments individuels" et si "le premier consacrele triomphe des forts "nous royons comment le second "tend a fortilier les faibles dans leur lutte contre les forts" (2).

Les faits ici ne nous font point défaut davintage. .11. Saurel a montré, pour la France. que "l'álimination progeressive des banques locales qui, an l'ital actued des conditions économiques. apparait en yuelque sorte comme fatales pourait, sans doute, par une modilication conve-

(1) Edouard Dolléans, lie l'.lcedparement, These Paris, 190:2, p. 3.

(2) Bourguin, op. cit., p. 133. 
nable de ces mêmes condilions être évitée et il a indiqué les efforts que déjà l'on avait tentés en ce sens (1).

Le mouvement s'est encore plus vigoureusement dessiné en Allemagne. Un certain nombre d'économistes, une partie de la presse allemande ont associé dans leurs attaques la féodalité industrielle et la féodalité financière ; ils ont cru pouvoir démontrer que les grands établissements de crédit, non moins que les cartels industriels avaient, sans merci, exploité la situation à leur profit. Mieux encore, ils ont en quelque sorte posé, pour la banque, la question préalable et exposé comment la Haute Banque dominant toute la grande industrie, c'était elle qu'il fallait réglementer d'abord. "Les directenrs des grandes banques cartelisées, disait M. Heymann en 1903 au Congrès des jurisconsultes allemands (Deutscher Juristentag), siègent en mème temps dans les administrations des cartels industriels. Ces directeurs de banques apportent les actions et les obligations des Sociétés à la Bourse, et comme ils détiennent la majorité des actions, ils sont les maîtres des assemblées générales! "Et M. Heymann terminait son rapport par le dépôt d'une motion tendant à une vigoureuse intervention législative, vœu qui finalement fut adopté mais non, tant s'en faut, à l'unanimité.

Et cette tendance que nous retrouvons dans les débats du Parlement, de la Commission des douanes, des chambres de commerce, dans la presse quotidienne, a trouvé une expression singulierement précise et significative dans cette grande enquè sur les cartels, ordonnée par le gouvernement allemand et qui, commencée le 14 novembre 1902, s'est prolongée jusqu'en 1904. On a entendu

(1) Saurel, op. cit., p. 126 et suiv. 
développer successivement les deux theses; on a soutenu que les cartels araient exploití ì la lois le consommatrur et l'ouvrier; d'autres ont établi que le seul olyjet de toutes les fusions et des groupements avail été d’amener un rendement plus rémunérateur du capital, une diminution du prix de revient, la suppression d'une concurrence ruineuse, la consolidation des relations de l'Allemagne avec l'extérieur. "Scule la concentration de nos forees, a déclaré le ministre des finances, haron Rheinhaben, nous mettra en état de conserver notre rang parmi les puissances." - Tous les travaux de la commission d'enquite n'ont daalleurs encore abouti à aucun résultat précis, ef un membre du Reichstag, I. Gothein, a pu dire que plus l’enquète s’était prolongée, moins la vérité sétait fait jour.

Quoi quil en soit, on voit par ces faits moins, éloignés de notre sujet particulier quil peut sembler au premior abord, comment, en Allemagne, la lutte est ouverte entre les partisans des deux tendances ot que ces dewx courants dont nous parlions sont vivaces et actifs. Sans doute, comme nous le verrons, un premier essai dintervention législative en faveur des petits banquiers n'a pas abouti; il a mème eu des conséquences diamétralement opposíes à celles quespéraient les légrishateurs (1. Mais les conditions économiquessont dans un perpétuel devenir ; journellement des faits nouveauxet importants viennent témoigner que la tendance à la concentralion dans les banques allemandes noa nullement ápuisi sa force: mais on doit aussi songer aux obstacles que peurent dresser sur la voie, aujourd'hui triomphale, des grands étabtissements et l’intervention législative ef l'ation des groupenents

(1) V. chap. II, L'Action Législative: le Börsengesetz. 
individuels. On annonçait dernièrement qu'un certain nombre de banquicrs provinciaux étaient sur le point de s'unir pour fonder à Berlin, un organe central analogue à notre Sociólé de Cirédit Industriel et Commercial qui, comme on le sait, est bien plutôt l'alliée que la concurrente des banques locales. Qui pent prévoir si demain l’évolution des banques allemandes ne se manifestera pas sous de nouveaux aspects qui viendraient briser les formules trop rigides lans lesquelles nous voudrions lenfermer et nous forcer à réviser des jugcements hàtifs et prématurés? 
PREMILRE PARTIE

ÉTUDE SYNTHÉTIQUE DU MOUVEMENT DE CONCENTRATION 



\section{CIIAPITRE PRENIER}

\section{LES BANQUES ALLEMANDES DE 1850 A 1904. - LE MOU- VEMENT DE CONCENTRATION ; TABLEAU GÉNERAL ; RÉSULTATS DENSEMBLE.}

Nos grandes banques, écrit un économiste allemand, ne peurent nullement prétendre à une antiquité respectable. Toutes sont une création de la seconde moitié du $\mathbf{x i x ^ { e }}$ siècle (1).

Jusque vers 18300 , nous ne trouvons en effet, en Prusse, à còté de la Preussische Bank, que quelques banques particulieres d'émission, - dans les Etats secondiares, In certain nombre de banques privées qui réunissent les opérations les plus diverses et répondent amplement aux besoins de l'industrie el du commerce ì peine naissints.

A cette date, l'économie de l'Mllemagne est encore presque exclusivement agricole: les swerres de l'Empire, lémiettement du territoire en petits Etats aux innombrables réglementations, systemes de donanes et d'impoots, ont retardé diun demi-siècle le développement industriol et commercial du pays.

Vers 1850 , les conditions économiques de l'Allemigne

(1) Fleischbammer, Contralisution in Bankusen in Jeutschland sckmollers Jahert., 1901, t. XXV. 
subissent des modifications profondes. A mesure que s'accroît la population, à mesure que s'accomplit l'évolution vers la grande industrie (1), le besoin d'unité s'af. firme avec plus de force, les barrières deviennent plus gênantes; la croissante unification douanière qui aboutit en 1851 au Zollwerein est l'expression de ce besoin. C'est vers cette époque que se fondent les premières banques par actions: à Cologne, l'A. Schaaffhausens'cher Bankverein en 1848; à Berlin, la Discontogesellschaft en 1851, conçues toutes deux sur le modèle du Cródit Mobilier des Pereire; un peu plus tard, la Bank für Handel und Industrie à Darmstadt (1853), à Berlin, la Mitteldeutsche Credit Bank, la Berliner HandeIsgeseltschaft (1856) (2).

Le but que vont se proposer ces banques nouvelles est aussi général que possible: Betrieb von Bankgeschäften aller Art. Elles voient très clairement et dès le début, à quels besoins elles vont avoir à répondre et que leur place est à la tête du mouvement industriel et commercial qui déjà se dessine vigoureusement. Voici comment s'exprime Ie premier rapport de la Darmstädter Bank:

“La Banque n'a nullement pour mission d'aider à l'agiotage et d'engager son capital dans des spéculations improductives. Tout au contraire, elle est appelée, au moyen de ses ressources personnelles et par la mise en valeur des fonds qui lui sont confiés, à créer de grandes et solides entreprises, à favoriser de toutes ses forces le développement de l'industrie allemande et à aider l'esprit d'entre-

(1) De 1826 à 1850 on ne compte que 102 fondations de sociétés par actions avec un capital global d'environ 639 millions de marks.De 1851 à 1870,295 fondations avec un capital de 2.404 millions de marks. (IV. Sombart, Das moderne Kapitalismus, II, p. 16).

(2) V.Model-Loeb, Die Grossen Berliner Effektbanken, p. 3 ; Poschinger, Bankwesen und Bankpolitik in Preussen, II, p. 214. 
prise el les capitamx à trouver, sulant les nóresités du moment, le droil elemin. Elle doil, tant à l’intérienr du pays qu'a l'étranger. favoriser l'exportation des produils allemands et faciliter les relations de l'industrie nationale avec te marehé linancier. Elle a lo droit et le devoir de fournir les capitaux disponibles à ceux qui, dans dautres branches de lindustrie, penrent en aroir besoin et par ces fréquents cechanges vivilier et fortifier larlivite industrielle. Elle est anssi antorisio a participer anx grandes entreprises industrielles. aux fondations de socicités, aux emprunts d'Etats, a faire rentrer dans son domaine le placement des fonds étrangers. En un mot, point de limite à son activité. "

C'est bien une nouvelle phase qui va s’ouvrir pour l'économie allemande. C'est anssi le premier coup porté à la prépondérance des vicilles places comme Hambourgo et Francfort "que d’anciennes maisons, à qui un crédit hors de pair donnait une situation de premier ordre, faisaient participer à l'ensemble des opérations d'arbitrage qui formaient alors un des ohjets escontiols de lactivité des banquiers" (1).

Ce n’est pourtant point de entto époque que dato le véritable essor des banques allemandes. Au commencement de 1870 , on ne comptait eniore en Allemagne, on dehors des banques d'émission, gur 11 banques par actions, dont 4 it Berlin. Leur capital veres atait de 32.110.000 thalers: celui des 29 banques d'ómission de $\$ 1.300 .000$ thalers $(2)$.

La fondation des banques par actions, comme celle des sociétés par actions, pestait encore subordonnér à liantorisation gowernementale et lour fonctionnement ibat

(1) R.-G. Levy, Les marches finamciers de lillemarne. Rerne div Deux-llondes, 15 novembre 1 s. $\%$.

(2) Max Wirth, Handbuch des Bankwesens. 
soumis à une surveillance étroite. La méfiance des Etats, qui craignaient, soit la concurrence que ces banques nouvelles allaient faire aux banques d'émission sur le terrain des opérations courantes, soit leur peu de solidité, faisait que cette autorisation était le plus souvent refusée.

Les fondateurs repoussés de Berlin, de Munich et des autres grandes villes, ou bien cherchaient à tourner la loi, - en Prusse par exemple, par la fondation de sociétés en commandite par actions qui n'étaient pas soumises à proprement parler à l'autorisation préalable, - ou bien s'adressaient aux petits Etats, d'où la fondation de banques provinciales (Schlesischer Bankverein (1856), Allgemeine Deutsche Credit Anstalt (1856), Hannoversche Bank (1857), Ostbank für Handel und Gewerbe (18:j7)...) que les grandes banques trouveront plus tard solidement établies et étendant sur toutes ces régions un réscau serré de succursales. C'est ainsi que la Bank für Handel und Industrie se vitrefuser par le Sénat l'autorisation de s'établir à Francfort : elle s'installa à Darmstadt à trois quarts d'heure de là. La Discontogesellschaft fut fondée sous la forme d'une société en commandite par actions (1).

Cette législation restrictive constituait un grave obslacle au développement des banques allemandes. La loi du 11 juin 1870 leva ces difficultés: elle étendit le droit commercial à toutes les sociétés, supprima l'autorisation et le droit de surveillance gouvernementales qu'elle remplaça par une série de dispositions légales : publicité plus rigoureuse, responsabilité plus grande des directenrs et administrateurs, etc...

Cette législation plus libérale fut le signal de la création d'un nombre considérable de sociétés par actions; les

(1) Voir Adolph Weber, Depositen und Spelulationsbanken, p. 48. 
banques qui nous intéreseent partieulièrement. se multiplient: la [rentache Bank (1), la Rheini-che Credit liank, la Breslaur Discontobank sont fondés liv 1870: puis viennent la Bergisch-Mïkische Bank en 1sil. la Dresdner Bank, l'Essener Credit Instalt en 1872. Ia Mitteluheinische Credit Bank en 1573, pour ne citer que les plus importantes. En 1875 . on comptait dóji dans les 62 villes de l'Empire où la Reich-bank arait un établissement 97 banques par actions el 33 -uccursales de banques.

Ausi bien cette année 1 $\$ 00$ marque-t-elle une étape dicisive dans l'histoire de l'Allemagne. La gnerre franco-allemande et la proclamation de l'Empire en 1871 réalisent l'unité politique et -urtout l'unité admini-trative at économique yni. seales, vont rendle posible ce prodigieux essor de lindustrie el du commerce allemands qui restera l'un des traits saillants le la fin du xix sieclen,2. La complete liberté de circulation assuríe a chacun, le commencement de ligislation commune sur bien des points, lounitr. presque réalisée matiquement, de lémision fiduciaire 3). la quasi-unitŕ postalé f). surtout l'mité moné-

(1) La fondation de a Deutsche Bank était dejit commencée sous l'ancienne lézislation.

2) G. Blondel. L'esor industriel et commercial th peuple allemand, p. 35 .

(3) Sur 3't banques d"emission existant au moment de la promultation de la loi du l't mars 1 Tij crént la heichsbank, 17 seulement accepterent les nourelles con litiuns qui leur ritient olfertes. Line série de mesures suppression des foltes coupures. interdieting aux ro. tenpricat banken de fure les opritions en dehors de leur circonscription, oblization pour elles liavir un bureau de rembursement a

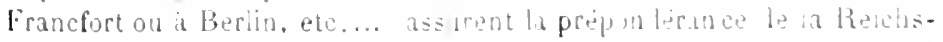
bank et préparent liuite. Aujourdhui il ne reste plus en Altemane que 0 banques d'émission.

(', La constitution de l'Empire allemand VI, art. $15-51$ ) créa une 
taire (1), tels furent les premiers résultats de la centralisation politique qui provoquèrent aussitòt l'éveil de l'activité el la mise en weuve des forces productives du pays tout entier. Le nouvel Empire se couve de manufactures, d'usines, de fubriques de toutes sortes. Quant aux banques, elles furent, a-t-on dit. les promoteurs et les bénéficiaires du nouvel ihat de choses : ce furent elles qui avancèrent les sommes nécessaires à la transformation et à la construction des usines (2). L'ensemble des opérations de la Discontogesellschaft qui atteignait fin 1869 un total de 2.6 .5 millions de marks, saute en 1871 à 4.041 , en 1872 à 7.179 millions de marks; de 761 a $1.33 \%$ millions de marks. La toute jeune Deutsche Bank augmente son capital de 17 milions de marks en 1872 , de 14 millions en 1873 et le total de ses opérations passe de 948 à 2.891 millions de marks en ces deux seules années.

La crise de 187:) survintqui fil lisparaître un certain nombre de banques, - "celles-lì qu’avait créées un en-

administration postale unique pour toute l'Allemagne, sauf la Bavière et le Wurtemberg qui ont conservé leur autonomie postale et télégraphique. La fusion des postes et télézraphes ful terminée le $1^{\text {er janvier }}$ 1876.

(1) "En 1871, il n'existait pas moins de 66 coupures différentes de monnaies d'argent et 17 coupures de monnaies d'or. L'un des premiers soins du govveruement impérial fut d'user des pouroirs que la constitution lui avait dunnés en classant parmi les matières fédérales la législation et la haute surreillance du système les monnaies, pour réaliser enfin l'unité monétaire de l'Allemagne. La réforme fut opérée par deux lois: la premiere date du í décembre $18 \% 1$, relative à la fabrication des monnaies d'or impériales; la seconde du 9 juillet 1873, relative aux monnaies d'argent et a la question de l'étalon. "Arnauné, La monnaie, le crédit et le change, p. 258.

(2) Blondel, op. cit., p. 482 . 
thousiasme débordant " ?(1) et qui ne servaient qua laigiotage et i laspéculation. Celles qui répondaient ì un réritable besoin áconomique non seulement supportirent onragensement la erise, mais, comme ha Dutsche Banket la Dresdner Bank par exemple, ensortirenthortitiées elagrandies: on peut déji entrevoir la formation de cettr sorte d'oligarehie de banquesqui va de plus s'emparer du marché, dont la force et l’importinee śaceroissent sans cesse. et qui linalement va concentrer sos opérations dans la capitale de l'Empire.

De tous les Etats qui composaient le nouvel Empire, la Prusse, qui renait de réaliser l'unité politique comme elle avait jadis réalisé l'unití donaniore, allait sassurer à cóté de l'légémonie militaipe, lepremier rang au point de vue économique. Le développement de la capitaleentlà comme ailleurs son oflet inévitable. Berlin, dont la population passe de 377.870 habitants en 1861 à 824.580 en 1871 , it 1.112 .304 en 1850, eapitale politique et administrative, siège de la Reichsbank, devient le grand marché économique et financier de l'Allemagne : la centralisation des grandes affaires $y$ suit la centralisation zouvernementale. Francfort perd définitivement son antique prépondérance: en $187 \%$, on compte à Berlin 2 't banques ou banquiers privés, 12\% seulement à Francfort, :00 it llambour's.

Les banques, qui, nous larons vu, eurent d'abord pour point d'attache les grantes villes de la Confidtération, durent naturellement ehereher it prendre pied tans la nouvelle eapitale et bientit. comme lagence tu Cródit Lyounais a Paris. leur filiale a Berlin devint le siege et le centre principaus de lours oprerations: lel ent le cas pour

(1) U. Varschaner, Physiologie der deutschen Banken, p. 'ú. 
la Bank für Handel und Industrie qui vient s'établir à Berlin dès 1871, la Mitteldeutsche Credit Bank (1873), la Dresdner Bank (1881), l'A. Schaaffhausens'cher Bankverein (1891) et enfin la Commerz und Disconto Bank (1898).

En 188 g le Reutsche OEkonomist comptait 71 banques de crédit représentant un capital de 723,95 millions de marks : le capital des banques berlinoises seules, représentait 326,74 millions de marks.

En 1890, on comptait 92 banques: 15 à Berlin avec 507,45 millions de marks de capital, 76 en province avec un capital de 546,88 millions de marks.

C'est à partir de cette date que commence le mouvement de concentration dans les banques allemandes et depuis ses progrès ont été singulièrement rapides et continus.

Les anciennes grandes banques provinciales devenues, au mème titre que la Deutsche Bank, la Discontogesellschaft, la Berliner Ilandelsgesellschaft, les grands Instituls berlinois, voulurent, animées du sentiment de leur puissance, désireuses d'étendre le champ de leur activité et le cercle de leurs opérations, poussées aussi par une concurrence plus vive, recommencer le chemin parcouru, mais cette fois en sens inverse, et de Berlin rayonner sur toute la province, en drainer l'épargne, pomper les capitaux flottants : la centralisation avait préparé les voies à la concentration.

Mais le particularisme provincial restait vivace. Aujourd'hui même encore, quoique déchues, Francfort et Hambourg ont une autre importance et une autre physionomie que les places de Lyon ou de Marseille. Les grands établissements berlinois allaient trouver dans les banques locales de solides concurrentes et de rudes adversaires; d'où le caractère particulièrement original que va prendre cette 
lutte qui se terminera d'ailleurs par te triomphe inconlin. table des grandes banques, grace a l’intluence de callses particulières, économiques, législatives et linancierres yue nous aurons ì examiner. Pour le moment, nous nous hornous i domner le résultat d'ensemble aupuel a léji abonti le mouvement de concentration.

Pour atteindre le but qu'elles s'étairnt proposé, les grandes banques allemandes durent, tout diabord, socenper de renforcer leurs moyens d'action, cest-ì-dire augmenter leur propre capital : le tableau suivant montre les principales augmentations de capilal des banques berlinoises depuis leur fondation :

\begin{tabular}{|c|c|c|c|c|c|}
\hline & \multirow{3}{*}{$\begin{array}{c}\text { Date } \\
\text { de } \\
\text { foudation }\end{array}$} & \multicolumn{4}{|c|}{ Capital-actions } \\
\hline & & Capilal & & & juillet \\
\hline & & fondation & 1896 & 1900 & 1905 \\
\hline A.Schaalfhausens'cher Bankverein. & . 1848 & 15,6 & 60 & 100 & $12: j$ \\
\hline Discontogesellschaft. . . . . . . & $18: 11$ & 30 & $11 \%$ & 130 & 170 \\
\hline Darmstiidter Bank . . . . . . & $18 \% 3$ & 17,1 & so & $10 \%$ & $15 \%$ \\
\hline Berliner Handelsgesellschaft. . & $18 \% 6$ & $4 \ddot{3}$ & 80 & 90 & 100 \\
\hline Milteldentsche Creditbank. . & 1556 & 21 & 30 & 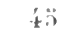 & 40 \\
\hline Deutsclse Bank (1). . . . . & $1 \times 70$ & 13 & 100 & $1 \breve{50}$ & 180 \\
\hline Commerz und biscontobank. . & 1870 & 30 & 30 & 50 & $8 i i$ \\
\hline Dresdner Bank. . & 1872 & 9,6 & 83 & 130 & 160 \\
\hline National Bank. ..... & $18 \times 1$ & 20 & 40 & 60 & so \\
\hline Berliner Bank . . . . . . . . & 1889 & $\ddot{z}$ & 20 & +2 & $"$ \\
\hline Total. . . . . & • & 211,3 & bii & 902 & 1099 \\
\hline
\end{tabular}

C'es élévations de capital se constatent dans l'ensemble des banques allemandes: (millions de markis).

(1) La demière a-semble genirate vetulne lonj de la beutsche

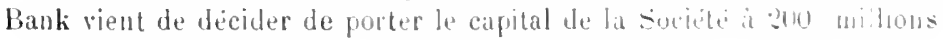
de marlis. 


\begin{tabular}{|c|c|c|c|c|c|c|c|}
\hline \multirow[b]{2}{*}{ Années } & & & \multicolumn{3}{|c|}{ Ensemble des banques allemandes } & \multirow{2}{*}{$\begin{array}{c}\text { Banques } \\
\text { Nombre } \\
-\end{array}$} & \multirow{2}{*}{$\begin{array}{l}\text { berlinoises } \\
\text { Capital }\end{array}$} \\
\hline & & & & Nombre & Capilal & & \\
\hline 1890 & & . & & 92 & 1054,33 & 15 & 507,45 \\
\hline 1895 & & . & . & 94 & 1134,82 & 16 & 626,86 \\
\hline 1900 & & & & 118 & $1959, \div 5$ & 18 & 1019,92 \\
\hline 1901 & & . & & 125 & 1959,29 & 16 & 1015,80 \\
\hline 1902. & & . & & 122 & 1980,59 & 15 & 1022,80 \\
\hline 1903 & & . & & 124 & 1989,96 & 15 & 1019,40 \\
\hline 1904 & . & . & & 129 & 2066,54 & 12 & 1071,20 \\
\hline
\end{tabular}

Le capital des banques allemandes de crédit a donc augmenté d'une façon notable: leur nombre également d'ailleurs, qui passe de 71 en 1883 , à 92 en 1890 , à 129 en 1904 ; mais ce serait une grave erreur que de vouloir interpréter ces chiffres dans un sens contraire à celui de la concentration. Le mouvement de concentration, nous le verrons en effet, n'a pas pris en Allemagne les mêmes formes qu'en Angleterre ou en France par exemple: il ne se manifeste pas seulement par la disparition des banquiers privés ou des banques locales, remplacés par les succursales des grands établissements. Ce n’est là qu'un des aspects du phénomène de concentration qui se poursuit en Allenagne sous les formes les plus variées: or, l'une de ses principales caractéristiques est de laisser le plus solvent aux établissements absorbés, fusionnés par les grands Instituls, facteurs de la concentration, leur personnalité propre et une autonomie formelle. Nous assistons beaucoup moins à la prédominance de quelques rares établissements, concentrant entre leurs mains et sous une mome raison sociale la majeure partie du trafic de Banque - , comme chez nous le Crédit Lyonnais, la Socićté Générale é le Comptoir National d'Escompte, qu'ì une concentration ite forces sous la forme d'alliances, d'unions, de groupements de banques plus ou moins in- 
dépendantes l'un grand établissement directeur qui lus

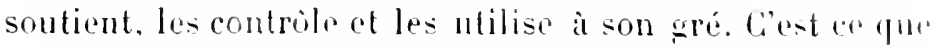
nous allons voir en esquissant rapidement la politipue des grands étibbiissements berlinois ris-ì-vis des banques privées et les banques par actions provinciales et en montrant les résultats auxquels a abouti cette politique.

a) Il est devenu de plus en plus difficile aux firmes particulières de lutter contre les massos de capitaux accumulées dans les grands átablissements: allssi, depuis une vinglaine dannées, les absorplions de natisons privées se sont-elles succédé, importantes et nombreuses. Dans son numéro dus janvier 1898 , le leutscher OEkonomist donnait un. liste de trente-ciny banques privées ayant ainsi disparu et depuis. ce mourement s'est encore accentué: la question des banques locales se pose aujourd'hui chez nos roisins arec plus d'acuité peut-ètre encore qu'en France (1).

Ces banques privées sont ou bien simplement absorbées par les grands établissements, "pompées " comme on dit maintenant en Allemagne el érigées en filiales proprement dites, ou bien transformées par euc en sociélés par actions commanditres: de toute façon, soit qu elles deviennent de pures et simples succursales, soit quelles survivent sous une autre forme, dles perdent leur indépendance et entrent dans le cercle d'action d'une grande banque.

Nous aurons à citer maintes fois le nom de firmes particulières, - et non des moindres - ainsi disparues ou transformées : la vieille maison Becker de lde iphigr, devemute en 1899 une filiale de la biscontogesellschaft, la maison

(1) V. Maturice Saurei, "p. ril. 
A. Kothschild de Francfort transformée en 1901 en filiale de ce même établissement; la maison $R$. Varschauer de Berlin, commanditée eu 1898 par la Darmstädter Bank pour 20 millions de marks et qui vient (190:) d'ètre absorbée complètement par cette banque. La maison Landau absorbée par la National Bank. En 1904, la Dresdner Bank absorbe l'antique et solide Banque Erlanger et fils établie depuis de longues années à Francfort et qui couvrait le pays d'un vaste réseau de succursales; la Deutsche Bank, la maison A. Molenaar de Crefeld, la banque Lazard Brach de Saarbrücken; la Discontogesellschaft, la maison I. Schultze el Wolde de Brême, la maison W. S. Vurburg d'Altona; elle transformait hier encore (décembre 1904) la première maison de la place de Mannheim, la puissante banque $W$. H. Ladenburg et fils en "Süddeutsche Discontogesellschaft ".

b) C'est cette même politique que nous retrouvons suivie par les grands établissements berlinois vis-ì-vis des banques par actions provinciales elles-mêmes: là encore, nous avons à enregistrer des fusions complètes, des absorptions totales d'une banque par l'autre; plus souvent encore, il arrive que les deux maisons gardent chacune une existence indépendante, l'une devenant le principal actionnaire ou le commanditaire de l'autre. Dans chacun de ces deux ordres d'idées, l'exemple de l'absorption de la Norddentsche Bank de Hambourg (au capital de 60 millions de marks) par la Discontogesellschaft en 1895, - et l'exemple de la participation de la Deutsche Bank dans la Bergisch Märlische Banti (capital en 1898, 40 millions de marks) et le Schlesischer Lanhverein (capital 27 millions de martis), sont devenus classiques en Allemagne.

Les banques berlinoises elles-mêmes ne sont point à 
l'abri drece absopplions par des rivales plus puissantos el plus actives, témoin la fusion tre la Doubshon Gonossenschaftsbank an capital de 30.000 .000 marlis avec la Dresilner bank, réalisée en 190', et plus récemment cncore, janvier 1903 , l'absorption de la Berliner bank an capital de 42.000.000 marks far la Commerz umol Disconto Bank.

En 1898 , le Deutsche OElonomist comptail li banques par actions totalement absorliés: an 1902 et 1903, 10 atutres d’un capital global do 6s..307.300 marks (1) en 1904, g banques par actions dont roici l’ónumeration, disparaissent:

Capilat-Actions

Marks

Berliner Bank... . . . . . . . . . 42.000 .000

Deutschen Genossenschaftshank . . . . . 30.000 .000

Börsen Handels Verein . . . . . . . . . . . 3.000 .000

Westdeutsche Bank. . . . . . . . . . . 9.1000.000

Oberheinische liank. . . . . . . . . . 17.000 .000

Paderusteiner lankrerein . . . . . . . . 1.500.000

Dortmunder Bankverein . . . . . . . . 10.801 .000

Niederrheinische Bank. . . . . . . . 21.000.000

Trierer Bank. . . . . . . . . . . . . 2.000 .000

136.001 .000

(1) Yous donnous la liste de ces banques absorbées en 1902-1903.

Capital-Actions

Marks

1 Barmer llaudelsbank . . . . . . . . . . 6.375 .000

2 Credit und Depositen Bank Zweitrücken' . . 1.000.000

3 Credit bank Recklinghausen. . . . . . . . . 3.1110 .000

\& Kommand-Gesellsch. A. Molenaar et c. . . 1.200.000

5 Bank für Rheinland und Westfaten . . . . . 10.0001.000

6 Kölnische Wechsler unıl Cummissions B. . . . 12.000.000

7 Bank für suddentschland. . . . . . . 15.62.300)

8 Dïsseldorfer Bankrepin. . . . . . . . 9.000.000

9 Coblenzer Bank . . . . . . . . . . . . 2000.000

10 Euskirchener Volksbank. . . . . . $=\frac{5.000 .000}{5.307 .300}$

Les quatre premipres de ces banques ont éta absorbers pill la Deulsthe

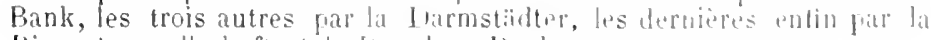
Discontogesellschaft et la lJresdner Liank. 
En revanche, celte mème année 1904, il s'est fondé 14 banques nouvelles:

Capital-Actions Marks

Bank für Chile und Deutschland . . . . . . . 2.500.000

Brasilianische Bank für Deutschland. . . . 10.000.000

Creditverein Neviges . . . . . . . . . 1.500 .000

Crefelder Bank. . . . . . . . . . . . 1.500 .000

Emder Bank. . . . . . . . . . . . 1.000 .000

Hamelner Bank. . . . . . . . . . . . . 1.800 .000

Lüdenscheider Bank . . . . . . . . . . . . 1.000 .000

Barmer B. f. Handel und Gewerbe . . . . . . . 2.100.000

Credit Verein Rosenheim . . . . . . . . . 1.000 .000

Oberfränkische Bank . . . . . . . . . . 1.800 .000

Trierische Volksbank . . . . . . . . . . . 2.000 .000

Weissenfelder Bankverein. . . . . . . , 1.209 .000

Wurzener Bank . . . . . . . . . . . . . . 1.000 .000

Pforzheimer Bankverein. . . . . . . . . . . $\frac{3.500 .000}{31.009 .000}$

Nous avons donné ce tableau pour bien montrer qu'il ne faut point juger des progrès de la concentration d'une façon absolue, uniquement d'après le nombre total, croissant ou décroissant, des banques dans le pays. De ces 14 banques nouvelles, en effet, 11 furent fondées avec l'appui des grands établissements berlinois ou sont commanditées par cux et rentrent ainsi dans leur sphère d'action.

C'est là le deuxième aspect de la politique pratiquée par les grandes banquues vis-à-vis des banques par actions; à còté des fusions complètes, des absorptions totales nous trouvons des fusions imparfaites, des absorptions partielles, peut-on dire, réalisées de diverses façons, par commandite, par achat ou échange d'actions et qui, tout en laissant aux banques ainsi agglomérées leur raison sociale et une liberté plus ou moins complète, ne les mettent pas moins 
sous la dépendance des grands rablissements ot les lont marcher à leur suite. Lensemble de ces sucenrsales al'un caractire tout à fait particulier constitue ce que nous arons appelé les groupes de hanques. Coest surtout dans colte direction que parait seatre dirigé l’activité des banques berlinoises. próoceupées depuis quiuze ans l'étendre leurs relations, leurs alliances, leurs participations dans les banques secondaires.

Ces dernières à leur tour, ont suivi la mèmı politique et se sont efforcérs dans lear rayon d'iuction propro de multiplier également les participations et les unions avec les banques locales, de sorte que. linalement, un groupe de banques nous apparait comme une srive de grouprments régionaux réunis, concontrés suls: le directiost unique din grand établissement aud capitaux considerebles et financisrement intiressi. directem"nt ou indirectoment, it chacun des éléments $(1)$.

Parfois mème, entin, ciest entre deux établissements de force semsiblement exale, tous denx chef's de groupe, que nous voyons se conclure des alliances, sortes de cartels de banque, qui vont concentrer dans une meme activité des capitand formidables: annsi la communamté

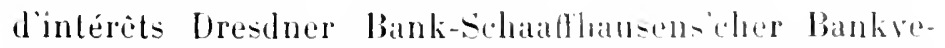
rein. conclue en décembre 19113, pour 30 ans, yui rémit un

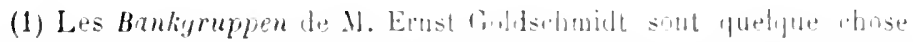

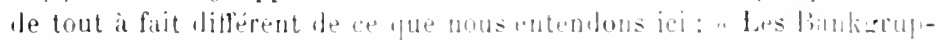
pen, dit-il (p. 6). sont des uninus de hanques pour la conduite conninune des aflinires. Letur essence onnsinte en ce fait que phosiends hall-

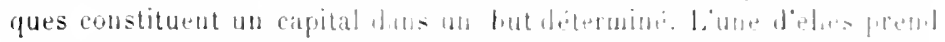

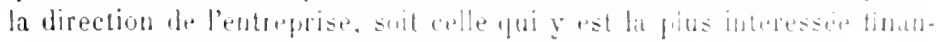
cièrement, soit celle qui peut le mieux surveiller e marehr... 0:

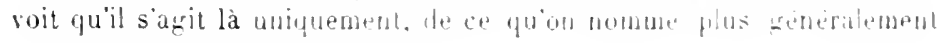
un consortium de bangues. 
catpital de $28 \%$ millions de marks, plus (fin 1904)64 millions de marks de réserves, soit un total de 349 millions de marks.

A cette lutte d'influence et de capitaux sans cesse engagée sur quelque point de l'Empire, les banques allemandes ont apporté une ardeur singulière. Citons en passant cet exemple caractéristique que nous trouvons dans la Neue Hamburger Zeitschrift du 20 juin 1905 sous le titre significatif de Combat de Banques à Königsberg : "La Deutsche Bank et la National Bank d'un côté, la Handelsgesellschaft, la Darmstädter Bank et l'A. Schaaffhausens' cher Bankverein de l'autre, ont fait hier une demande si vive des actions de l'Ostdeutsche Bank que l'offre ne suffisant plus, on dut effacer la valeur de la cote. Le premier groupe désire,comme on sait, fusionner l'Ostdeutsche Bank avec la Norddeutsche Credit Anstalt, tandis que le second veut l'incorporer à l'Ostbank de Posen »

Aussi ne reste-t-il plus aujourd'hui en Allemagne qu'un petit nombre de maisons indépendantes ou isolées : la plupart des banques sont d'une façon ou d'une autre liées aux intérêts d'un groupe placé sous la direction d'une grande hanque : c'est à Berlin que viennent se réunir tous les fils de cette organisation très largement ramifrée. Nous nous trouvons dès maintenant en présence de quatre groupes nettement caractérisés, dont nous aurons à préciser plus tard et la physionomie propre et le mode de formation: 
1" Groupe de la Deutsche Bank. Ce groupe comprent: fin 1904

$$
\begin{aligned}
& \text { Capital-Actions Rínerves } \\
& \text { Marks }
\end{aligned}
$$

Deutsche Bank (Lerlin) 1). . . . . 180.000 .00075 .000 .1900

Bargisch Mäkirsche Bank (Eherfeld). $60.000 .000 \quad 1.639 .559$

Schlesischer Bankverein (Breslau). . $30.1000 .000 \quad 9.000 .000$

Hannoversche Bank (Manorre . . 2 $2.500 .000 \quad 3.292 .180$

Osnabrücker Bank (1)snabrïck) (2). . $\quad 8.000 .000 \quad 2.566 .000$

Hildesheimer Bank Hildesheim). . $\quad 8.000 .000 \quad 2.000 .000$

Duisburg liuhrorter Rank (Duisburs). $\quad 12.000 .000 \quad 79.031$

Essener Credit Anstalt (Esen (3. . $\quad 40.0110 .800 \quad 10.1111 .000$

Westfülischer Bankerein (.Hunster). $\quad 7.000 .000 \quad 1.621 .000$

Siegener Bank für Handel und Ge-

werbe (Siegen). . . . . . . \$ $4000.000 \quad 5: 5 \% .000$

Sächsische Bank (Dresde) . . . . $30.000 .000 \quad 6.539 .763$

Essener Bankverein (Essen) (4). . . $7.500 .000 \quad 8 \% 00000$

Oldenburgische Spar und l.eih. liank

(ildenburg . . . . . . .

$\begin{array}{lllr}\text { Emdener Bank (Emden) . . . . . . . . } & 1.000 .330 & 219787 \\ \text { Prival Bank zu Cotha . . . . . . . . } & 7.200 .000 & 1.103 .160\end{array}$

Mecklemburgische Hypoteken und
Wecheel liank sciwerin . . . $9.000 .000 \quad 3.750 .000$

Necklemburgische Spar liank Schwe-

rin). . . . . . . . . .

\begin{tabular}{rr}
3.000 .800 & 100.000 \\
70.000 .000 & 0.378 .819 \\
1.000 .000 & 609.671 \\
\hline 07.201 .9350 & 142.263 .990
\end{tabular}

1) La dernitre assemblée générale de la beutsche Bank a décids de porter le capital de la société à 200 millions de marks.

(2) LOsmabricker B. a porté son capital à 10.000.0100 marks pn 1905.

(3) L'Essener Credit Anstalt a porté son capital.en 1905. à í.000.000 marks.

(4) L'Essener Bankverein a porté son capital en 1905, à 10.000.000 marks. 
$2^{\circ}$ Groupe de la Liscontogesellschaft. Ce groupe contrôle :

\begin{tabular}{|c|c|c|}
\hline fin 1904 & Capital-Actions & $\begin{array}{l}\text { Réserves } \\
\text { Marks }\end{array}$ \\
\hline Discontogesellschaft (Berlin). . . . & 170.000 .000 & 57.592 .611 \\
\hline Norddeutsche Bank (Hambourg) (1) . & 50.000 .000 & 9.015 .000 \\
\hline \multicolumn{3}{|l|}{ Allgemeine Deutsche Credit Anstalt } \\
\hline A. Busse et Cie (Berlin). . . . . & 6.000 .000 & 86.870 \\
\hline Vogtländische Bank (Plauen). . . & 5.000 .000 & $2.620 ี .022$ \\
\hline Oberlausitzer Bank (Zittau). . . . . & 2.700 .000 & 620.000 \\
\hline Vereinsbank (Grimma). . . . . . & 3.500 .000 & 130.783 \\
\hline \multicolumn{3}{|l|}{ Rheinische Discontogesellschaft (Aix- } \\
\hline Bochumer Bank (Bochum) . . . . & 5.000 .000 & 308.000 \\
\hline Eschweiler Bank (Eschweiler). . . . & 600.000 & 81.043 \\
\hline Dürener Bank (Düren) (3). . . . & 600.000 & 130.000 \\
\hline Volksbank Geilenkirchen . . . . & 315.000 & 72.252 \\
\hline Oberbergische Bank $(O h l)$. . . . & 500.000 & 52.500 \\
\hline Eupener Credit bank (Eupen) . . . & 500.000 & 33.900 \\
\hline Barmer Bankverein (Barmen) (4) . & 46.140 .800 & 8.100 .000 \\
\hline \multicolumn{3}{|l|}{$\begin{array}{l}\text { Süddeutsche Disconto Gesellsch. } \\
\text { (Mannheim). . . . . . . . . . }\end{array}$} \\
\hline & 379.853 .800 & 118.976 .876 \\
\hline
\end{tabular}

(1) Le capital de la Norddeutsche Bank, entièrement possédé par la Disconto, ne figure pas dans le total.

(2) L'Assemblée générale du 4 août 1905 a porté le capital de la Rheinische Discontogesellschaft à 60.000.000 marks.

(3) Le capital de la Durener Bank a été porté à 1.000 .000 marks en 1905.

(1) Le capital du Barmer Bkv. vient d'être porté à 47.974 .400 marks. 
$3^{\circ}$ Groupe Diesdner Bank-A. Schaaflhausens'rher Bankverein:

$$
\text { fin } 1904
$$

Dresdner Bank (Dresde et Rerliu) . . Märkische liank (Bochum . . . . Rheinische liank (Miilheim) (t). . Oberschlesische Bank Beutlien). . Wültemberoische l.andesh. (Stuttgart). . . . . . . . . .

Oldenburgische landesbank (Oldenburg) (2). . . . . . . . . .

Mecklemburgische Bank (Schwerin) 3 . Rostocker Gewerbe Bank Rostock!. Neurorpomm. Spar und Creditb. (Stralsund). . . . . . . . . . .

Iand"räflich.Hessischel.andesb.(Homburg). . . . . . . . . . .

schwarzburgische l.andesb. (Sonders hausen) (́). . . . . . . . .

A. Schaaffhausens'cher Blix. (Cologne et Berlin). . . . . . . . . . . Osldeutsche Bank (Känigsberg) š . . Pfalzische Bank Ludwisshafen). . . Miltel heinische Bank Coblenz. . Mülleimer Bank (Mulheim). . . . Westfalisch. Iippische Vereinsb. (liie-

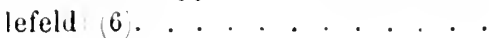

\begin{tabular}{|c|c|}
\hline Capital-Actions & $\begin{array}{c}\text { Réserves } \\
\text { Marks }\end{array}$ \\
\hline 160.000 .000 & 47.500 .000 \\
\hline 8.000 .000 & 220.000 \\
\hline 10.000 .000 & $1.090 .78 t$ \\
\hline 3.000 .000 & 320.000 \\
\hline 6.000 .000 & $9 \% .772$ \\
\hline 1.200 .1$)(10$ & $620.5 \%$ \\
\hline 2.0000 .000 & 243.211 \\
\hline 2.000 .000 & 137.096 \\
\hline 1.000 .000 & $\dddot{3} .93 t$ \\
\hline 1.000 .009 & 145.521 \\
\hline 1.000 .000 & 87.187 \\
\hline 123.000 .000 & 23.729 .761 \\
\hline $10.0(10.000$ & $8 千 0.061$ \\
\hline วั0.000.000 & 8.819 .813 \\
\hline 12.000 .000 & 577.226 \\
\hline 7.500 .000 & 308.205 \\
\hline כ. 000.000 & 187.339 \\
\hline $40 \div .700 .000$ & 83.160 .441 \\
\hline
\end{tabular}

1) Le capital de la liheinische bank a été porté en mai 1!105 à @1.000.1100 mark.

(2) Capitil nominal, 3.000 .000 marks.

(O) Ciapital nomiual, 5.000.000 marks.

(i) Capital nominal, 2.,000.00 marlis.

(j) LOstdeutsche bank a fusionese en 1955 arec la botbank fur Handel und Gewerl)

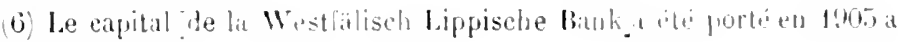
7.000.000 marks. 
$4^{\circ}$ Groupe de la Bank für Handel und Industrie (Darmstädter Bank): il comprend:

$$
\text { fin } 1904
$$

Darmstädter Bank (Darmstadt et Berlin).

Breslauer Discontobank (Breslau) . . Ostbank für Ilandel und Industrie (1)

(Posen). . . . . . . . . Bromberger B. fül Handel und Gewerbe (Brombers). . . . . . . Nordwestdeutsche Bank (Brème) (2). Würltembergische Bank (sluttgart). .

\begin{tabular}{rr} 
Capital-Actions & \multicolumn{1}{c}{$\begin{array}{c}\text { Réserves } \\
\text { Marks }\end{array}$} \\
154.000 .000 & 27.000 .000 \\
23.000 .000 & 1.260 .246 \\
8.000 .000 & 1.446 .000 \\
2.000 .000 & 20.000 \\
5.000 .000 & 245.029 \\
7.000 .000 & 1.440 .000 \\
\hline 201.000 .000 & 31.411 .275 \\
\hline 232.411 .275
\end{tabular}

Le Deutsche OEkonomist ( 22 juillet 1905) énumère encore trois autres groupes: nous les donnons également quoiqu’ils ne soient pas encore nettement caractérisés.

1) Groupe de la Mitteldeutsche Credit Bank à Berlin et à Francfort:

Capital-actions . . . . . . . 45.000.000 marks

Réserves . . . . . . . . $\frac{6.170 .000-}{51.170 .000-}$

La Mitteldeutsche Credit Bank est associéc aux maisons Meyer et Diss (Baden-Baden) et North, Kammeier et $\mathrm{C}^{\circ}$ (Essen).

2) Groupe de la National Bank für Deutschland (Berlin).

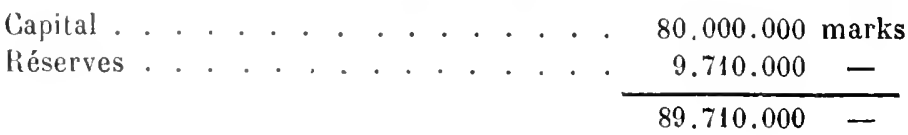

(1) Le capital de l'Ostbank a été porté à 18.000 .000 marks en 1905.

(2) Celui de la Nordwestdeutsche Bank à 7.000 .000 marks. 
La National Bank est intíressée à l'Orient Bank d'Athines et commandite la maison Erttel, Freybery et Cie it Leipzig.

3) Groupe de la Commerz und Disconto Bank ì Berlin, Hambourg el Kiel. Elle est intéressée a la maison J. Dreylus et Cie, à Francfort et au Ciredit und Spar Bank de Leiprig.

\begin{tabular}{cc}
$\begin{array}{c}\text { Capital } \\
\text { juillet } 1903\end{array}$ & Réserves \\
$\$ 3.000 .000$ & 7.300 .000 \\
$10.3 \% 0.000$ & - \\
\hline 93.350 .000 & 7.500 .000
\end{tabular}

102.850 .000

Si nous additionnons les capitaux et les réserves de ces ditférents groupes, nous arrirons au résultat suivant:

\begin{tabular}{|c|c|c|c|c|c|c|c|}
\hline & & & & & Capital-Actions & Réserves & $\begin{array}{l}\text { Total } \\
\text { markis }\end{array}$ \\
\hline $1^{\text {er groupe. }}$ & . & & . & . & 507.201 .930 & 142.263 .990 & $6 \div 9.467 .940$ \\
\hline $2^{\mathrm{e}}$ groupe & . & . & . & . & 379.833 .800 & 118.376 .870 & $\$ 98.232 .676$ \\
\hline $3^{e}$ groupe. & & & . & . & $40 \div .700 .0100$ & 83.100 .491 & $489.860 .+91$ \\
\hline $4^{\mathrm{e}}$ groupe. & . . & 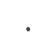 & . & . & 201.000 .000 & 31.111 .273 & 232.411 .275 \\
\hline $5^{\mathrm{e}}$ groupe. & - . & & . & & 千范000.000 & 6.170 .000 & 31.170 .000 \\
\hline $6^{\mathrm{e}}$ groupe. & . . & . & . & . & $80.009 .(1000$ & 9.710 .000 & 89.710 .000 \\
\hline \multirow[t]{2}{*}{ ie groupe. } & . . & & . & . & 95.330 .000 & 7..500.000 & 102.830 .010 \\
\hline & & & & & 07.รวั0 & 400.394 .632 & 2.113 .702 .382 \\
\hline
\end{tabular}

Soit un total de 2.113.702.382 marks en face dupuel les ressources propres desétablissements restés indépendants ne représentent plus guere que 3030 millions.

Engagies dans mo lutte de jour en jour plus difteile, sollicities de toutes parte. ces banques résisteront-elles longtemps encore it ce courant général de concentration? - Ne verrons-nous point diatres communatutés d'intérets, toujours plus considérables se produire el tinalement deux 
cartels gigantesques dominer le marché? - Ce sont là des questions qui viennent naturcllement à l'esprit quand on considère l'importance des résultats auxquels a abouti en ces quelques années le mouvement de concentration : les faits répondront.

Aussi bien, ce mouvement de concentration dans les banques allemandes dont nous venons de donner les résultats d'ensemble, a-t-il été et reste-t-il intimement lié à tout le mouvement économique et politique du pays luimême. Il nous faut entrer maintenant dans l'étude de ce mouvement et, pour bien saisir l'exacte physionomie du tableau de la concentration dans le trafic de banque en Allemagne, rechercher quelles en furent les causes et reprendre d'une façon plus analytique l'étude des moyens techniques qui servirent à la réaliser. 


\section{CHAPITRE II}

\section{LES CAUSES.}

Nous distinguerons deux sćpies de causes du mouvement de concentration dans les banques allemandes:

$1{ }^{\circ}$ Une caus ginirale, facteur premier de l'érolution, qui n'est, en somme, que l'expression originale prisedans le trafic de banque en Allemagne, par la tendance générale à la concentration que l'on constate dans tout le domaine capitaliste.

$2^{\circ}$ Des causes particulieres qui vinrent soutenir et précipiter cette tendance :

a) L'action législative ;

b) Les phénomènes internes à chaque établissement;

c) La crise de 1900-1901.

\section{1. - Cause génèrale.}

L'évolution économique moderne est caractériséc par une tendance générale à la concentration des eapilaux et des entreprises. La concentration dans le trafic de banque n’est done pas un fait iole : elle est farvorisée au contraire 
par le développement économique tout entier, comme aussi bien, ì son tour, elle vient réagir sur lui.

La cause générale de la concentration dans une hranche donnée du commerce ou de l'industrie, se présente ainsi sous un double aspect: une cause interne, tendance à la concentration propre à ce commerce on à cette industrie déterminés, et plus ou moins agissante suivant les conditions techniques de l'activité de cette branche de production; - une cause externe, expression des rapports plus ou moins étroits qui l'unissent à l'ensemble des phénomènes économiques et sociaux.

Si nous appliquons cette distinction à l'étude de la concentration dans le trafic de banque en Allemagne, nous nous trouvons en présence: $1^{\circ}$ d'une cause interne, technique, qui est la façon mème dont les Allemands comprennent et pratiquent la banque, façon particulièrement propre à favoriser la concentration, comme nous le verrons, en ce sens que les banques allemandes réunissent en un même orğanisme, des fonctions très différentes; $2^{\circ}$ d'une cause externe, économique, qui est le lien étroit qui unit en Allemagne, le commerce et l'industrie à la banque.

Sans doute, nous nous rendons compte de ce qu'une telle distinction présente d'absolu et d'arbitraire. Il est difficile, sinon impossible, de préciser dans quelle mesure la façon de comprendre el de pratiquer la banque a influé sur le développement économique du pays et conduit à cette coordination des différentes branches de l'activité ou dans quelle mesure au contraire le développement économique a obligé les banques à élargir la conception qu'elles avaient de leur rôle et à étendre leur cercle d'action. Nous ne songeons point à reprendre la vieille question 
de savoir si l'orane a créé la fouction ou si la fonction a créć l'organe. En réalité il y ent action et réaction continues et simultanées des deux phénomenes l'un sul l'autre; s’il est vai qu” "en général la forme de léchange correspond a la forme de production" (1), et "que dans une économie toute faite et quand on considire los phénomines acheve's, le mode d'échange pent ítre consilérí comme une consóquence du mode de production ". cela peut noitre plus vrai, "quand on consilere les devenirs et lis moyens de passage" (2).

Quoiqu'il en soit de ces discussions purement théoriques, et ces réserves une fois faites, nous étudierons pour la clarté de l'exposition, la cause générale lu mourrment de concentration du tratic de banque en Allemagne, sous chacun de ces deux aspects que nous avons imdiquís, technique et proprement économique.

\section{A. - La cause technique.}

Cette cause n'est autre que la fiçon mème dont les Allemands comprennent el pratiquent la banque.

Schaefte appelle les banques de son pays " des entreprises à tout faire " (Allerlue Entreprisen et sattler fait remarquer "qu'elles nont pour fonction essenticlle ni li paiement ni le crédit "(3). Les banques d'émission. les

(1) Rarl Marx, Misère de la philosophie. Qe idition, p. 10..

(2) G. Sarel, Introduction it l'conomie modeme, 1. litis.

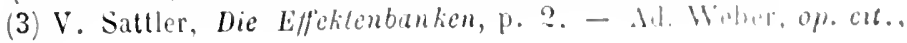
p. 4. 
banques hypothécaires et quelques banques de courtiers (1) (Naklerbanken) mises à part, la spécialisation des banques n'existe, en effet, pour ainsi dire pas en Allemagne: nous ne trouvons pas chez nos voisins, de banque ayant exclusivement le caractère d'une banque de dépôts et de crédit, comme chez nous Je Crédit Lyonnais ou la Société Générale, - ou celui d'une banque d’affaires proprement dite, comme la Banque de Paris et des Pays-Bas par exemple. A plus forte raison, la différence est-elle grande avec l'Angleterre où la spécialisation des banques est poussée à un haut degré. Les banques allemandes, elles, ne se sont point cantonnées dans une nature particulière d'opérations: toutes combinent dans une proportion plus ou moins grande les fonctions de banques de dépôts, de banques de crédit et de banques d'affaires (2).

"Le but de la société, dit le paragraphe 2 des statuts de la National Bank für Deutschland, est l'exploitation des affaires de toutes sortes: banque proprement dite, opérations financières, crédit, émissions, affaires industrielles et immobilières. "C'est là comme une formule de style que nous retrouvons dins tous les statuts.

Sans doute, la distinction établie entre banque de dépôts, banque de crédit et sociétés financières, présente un caractère trop absolu: c'est surtout une division théorique. "Dans la réalité, il n'existe plus de pures banques

(1) Les Maklerbanken sont des sociétés par actions qui s'occupent principalement de l'exécution des marchés à terme: il n’en existe que trois à Berlin.

(2) Sur l'activité générale des hanques allemandes, voir en dehors des ouvragres de Sattler, de Weber, de Varschauer déjà cités, A. E. Sayous, Banques de dejpôts, Banques de credit et Soeiètés finaneières; - Scharling, Bankpolittk; Handwörterbuch der Staatswissenschaften (Conrad) et Wörterbuch der Volkswirtschaft (Elster), art. Bank. 
de dépòts, il nixistre que pen do pures banques de crádit et les pures sociótís financieres ne sont qu'une assez rare exception. Une suule maison ou association réunit généralement ces trois branclues dans son activité tont en faisant à l’une, sinon à deuxd'entre elles, une place plus spécialement large" (1). Autrement dit, d'une facon générate et particulièrement en France, la distinction ne subsiste guère, nettement établie, qu'entre les attributions de dépòt el de crédit d'une part, et de l'autre les opérations linanciìres au sens striet du mot, les opérations de "Crídit Mobilier " pour cmployer lexpression courante en Allemagne.

Or c’est précisément l’intime confusion de ces attributions diflérentes qui caractériso les banques allemandes, les unes comme la bentiche bank ou la Dresdner bank par exemple étant des banquus de dépôts et de crédit autant que des sociétés finaucières, les autres, au contraire. comme la Bank für IIandel und Industrir ou la Discontogersellschait étant surtout des banques l'affaires.

Un aperçu général de l'organisation et de l'autivité des banques allemandes est ici nécessaire.

Cette confusion est le produit de l'érolution bistorique que nous avons esquissée. Arant 18:00, avons-nous vu, les Notenbanken se livraient ia coté de l'émission des billets à toutes les affaires courantes de banque : los premières banques par actions furent eréées pour subvenir anx besoins de limdustrie et du commerece naissants : ausoi,

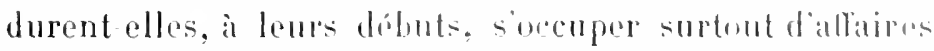

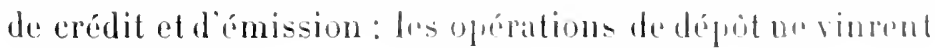

(1) Sayous, op. cil., p. 12. 


\begin{tabular}{|c|c|c|}
\hline \multirow{3}{*}{ 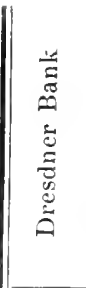 } & 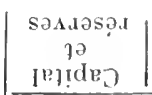 & 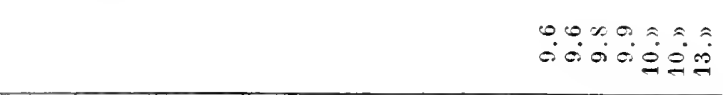 \\
\hline & syod?a & 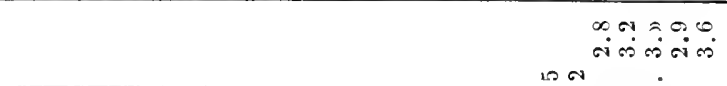 \\
\hline & 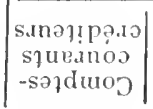 & 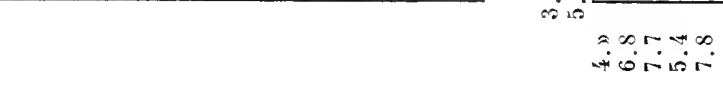 \\
\hline \multirow{3}{*}{ 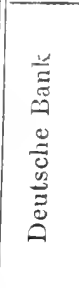 } & 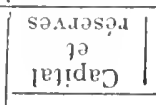 & 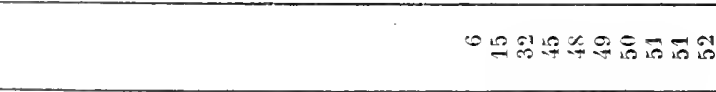 \\
\hline & s7odsa & 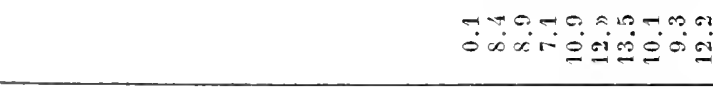 \\
\hline & 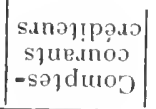 & 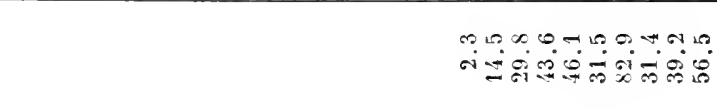 \\
\hline \multirow{3}{*}{ 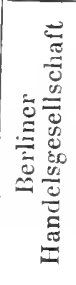 } & 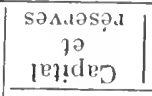 & 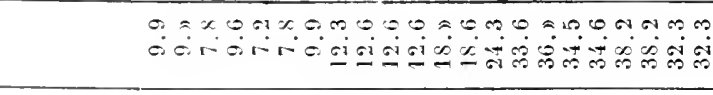 \\
\hline & spodą & \multirow{2}{*}{ 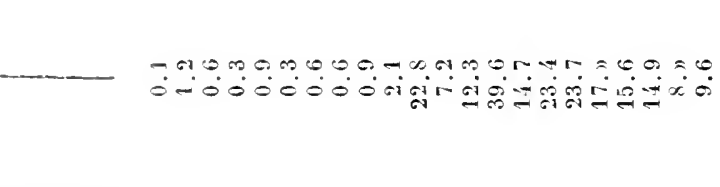 } \\
\hline & 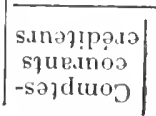 & \\
\hline \multirow{3}{*}{ 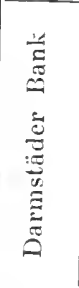 } & 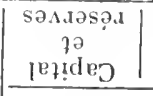 & 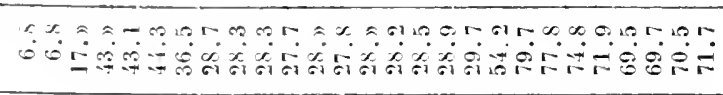 \\
\hline & şodą & 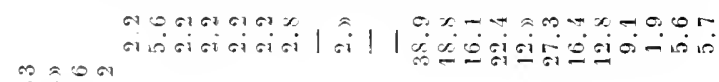 \\
\hline & 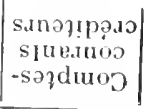 & 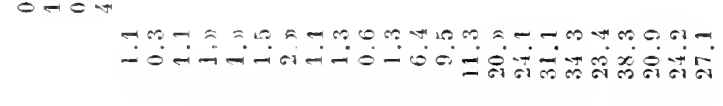 \\
\hline \multirow{3}{*}{ 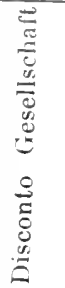 } & 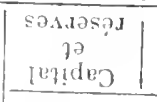 & 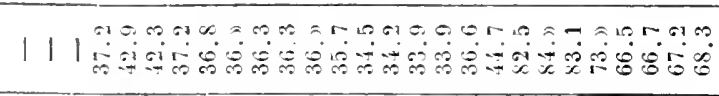 \\
\hline & spudag & 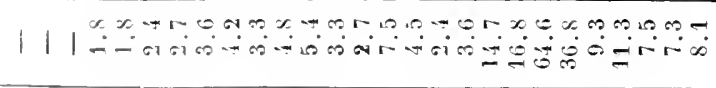 \\
\hline & $\mid \begin{array}{c}\text { s.tnaj!pa.da } \\
\text { sue.unos } \\
\text {-sajduoo }\end{array}$ & 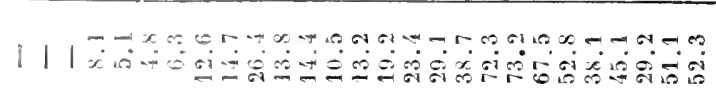 \\
\hline & 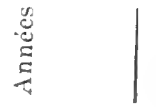 & \\
\hline
\end{tabular}


que plus tard s'y adjoindre (1). Le tableau ci-contre montre pour quelle faible proportion les dépots rentroul dius l'activité des ngrandes banques au début de leur essor. (loir ce tableau page $\breve{2})$.

Il faut noter également ici linfluence du caracterescermanique. "L'Allemand est investigateur" (2), il aime la spéculation et préfère aux fonds d'itat, les valeurs industrielles. Aussi ne place-t-il gruere ses fomds en dépots et jusquà ces derniôes anmés le montanl de ceux-ci dans les grandes banques atait relatirrment pen élur. Mais les dépòts n'en constituaient pas moins pour celles-ci un singulier rentorcement de leurs moynons l'action : aussi sc sont-elles efforées de les divelopper dans une plus large mesure el se sont-elles opposées a la fondation de banques particulières de dépòts sur le modile anglais $(3)$.

Le total des dépots et comptes créditeurs se chiffrait dans les grandes banques berlinoises, en millions de marks :

(1) "Le développement économique de l'Allemagne, en rendant né. cessaire une séparation frauche des banpues d'émission et les banques ordinaires eut pour effet le maintien, it colli de hangues lienission conçues dans un esprit moderne, de hamques conçus selon tes principes fort roisins des anciens; les oprérations de défuits salluerent bientùt aux opérations de creidit el aux opétations financines a le lien devint sans cesse plus intime a mesure qut le pays se developria, que l'activité s'accrut d Sayous, op. cut, p. 27!).

(2) Blondel, op. cit., 1, 27\%.

(3) V. Fleischhammer, art. cil. 


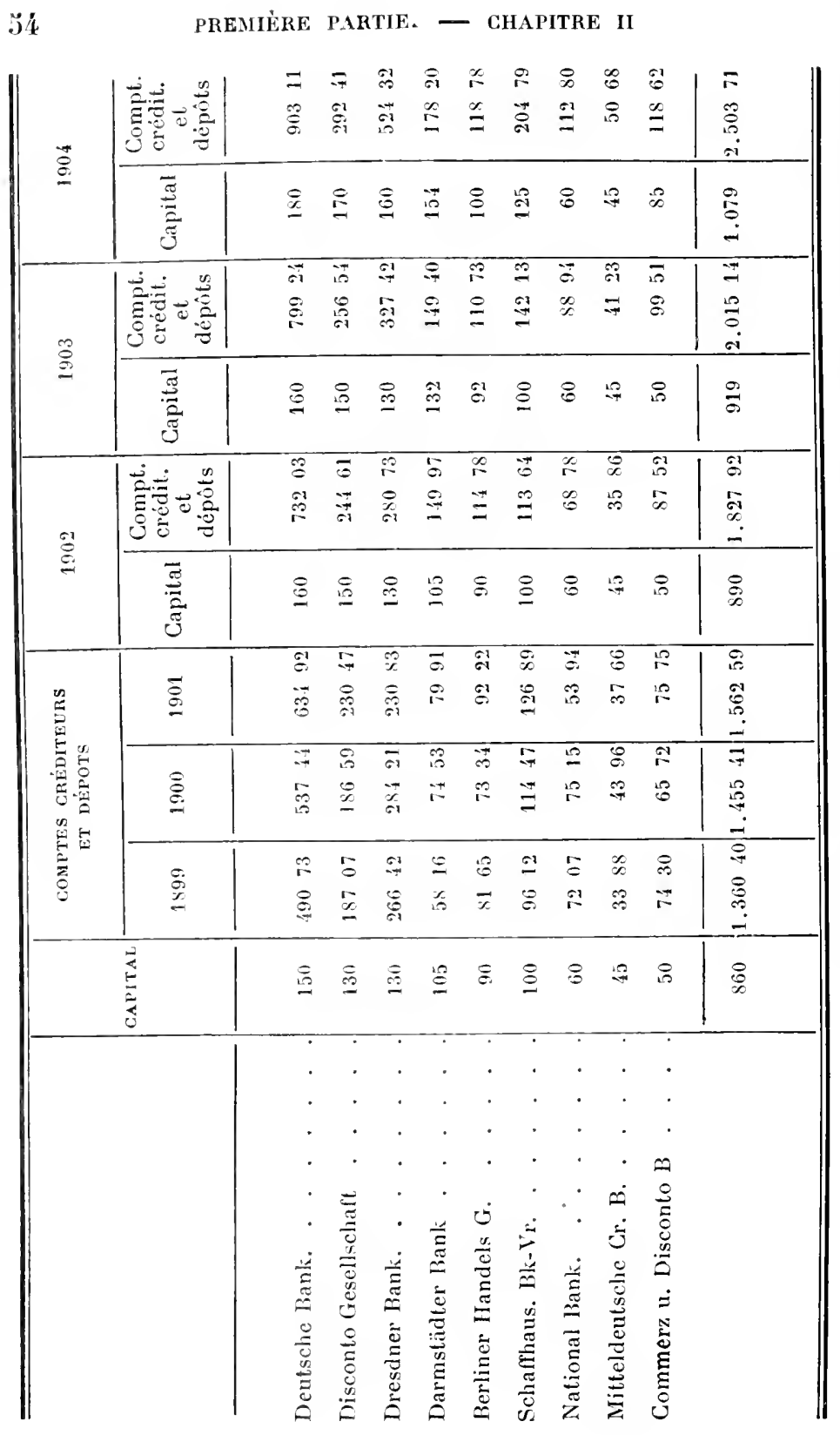




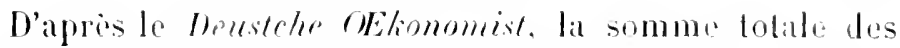
dépòts et comptrs credilrurs soilevait rn 1901. dans les 129 banques de réulit allomandes roprésentant un capital de 2.066 .336 .000 marks plus tis.380.000 marks de ré-

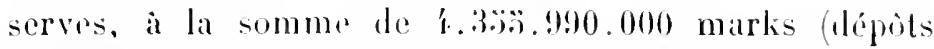
1.363 .963 .000 marks; comptes créditenrs 2.790 .027 .000 marks) contre:

\begin{tabular}{|c|c|c|c|c|c|c|c|}
\hline \multicolumn{5}{|c|}{ anneces } & banques & $\begin{array}{l}\text { rapital } \\
\text { marks }\end{array}$ & $\begin{array}{l}\text { résel ves } \\
\text { markis }\end{array}$ \\
\hline 1900. & . & & . & . & $11 x$ & 1.939.:348.000 & 390.931 .000 \\
\hline 1890. & . & & . & . & 92 & 1.081 .328 .000 & 187.880 .000 \\
\hline 1883. & & & . & . & 71 & $70: 3.000 .000$ & 90.847 .000 \\
\hline \multicolumn{5}{|c|}{ années } & \multicolumn{2}{|c|}{$\begin{array}{l}\text { depuits } \\
\text { manks }\end{array}$} & $\begin{array}{c}\text { comptes crètiteurs } \\
\text { marhs }\end{array}$ \\
\hline 1900. & . & & • & . & & 2.2 .000 & 2.130 .726 .000 \\
\hline 1890. & . & & . & . & . 40 & $1+.000$ & 883.106 .000 \\
\hline 1883. & . & & . & . & $2 ;$ & 2.2 .000 & .98 .500 .000 \\
\hline
\end{tabular}

On peut voir par ces chiffres, limportant développement quont pris en Allemagne les comptes courants et les dépóts, ees derniers seuls, saccroissant en dix ans

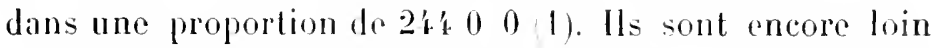
cependant de joner, dans lactivili des banques en lllemagne, le mème ròle quen Angletrere où socibtés financiores et banques privés ont en diprits phus de 20 milliards de franes et meme qui en France oil, all 31 décembre 190 t, nos arandes socirtás de eródit ditenatent (millions de francs)

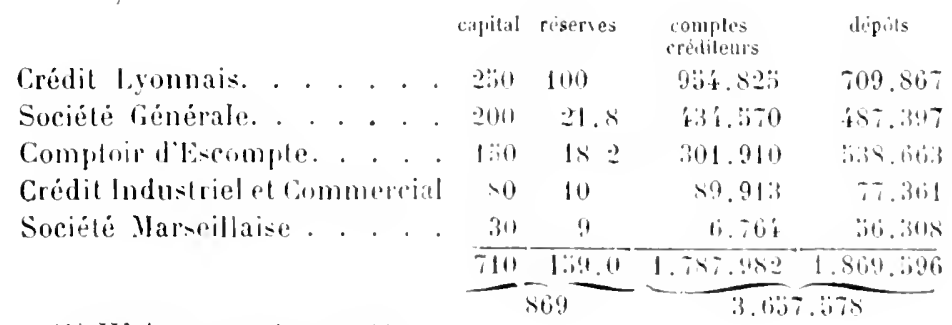

(1) Weber, op. cit., p. 3. 
Ceci peut expliquer pourquoi nos grandes banques n'allouent guère plus de $1 / 20$ d'intérèt par an à leurs déposants ì vue: en Allemagne, les banques recherchent ces mêmes dépòts à 2 et $30 / 0$. Voici, par exemple, les conditions de dépòts à la Beryisch-Märkische Bank (1):

Dépôts à vue ...... Intérèt de $20 / 0$

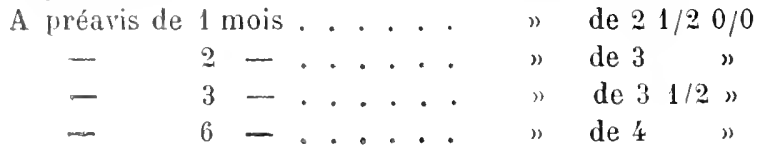

On comprend également maintenant pourquoi les banques allemandes ne pourraient distribuer les gros dividendes qu'attendent d'elles leur's actionnaires, si elles se contentaient de placer principalement leurs disponibilités en portefeuille commercial, comme font généralement nos grandes sociétés de crédit.

Le portefeuille des 71 banques de crédit allemandes représentait en $1883,453,3$ millions de marks - en 1900 (11 8 banques), 1.583 millions de marks, - en 1904 (129 banques) $1.773,7$ millions de marks dont 1075,6 pour les banques berlinoises. A cette même date (1904) le portefeuille de nos sociétés de crédit représentait:

1 Crédit Lyonnais.

2 Société Générale.

3 Comptoir d'Escompte

4 Crédit Industriel et Commercial.

¿ Société Marseillaise. . . . .

$$
\begin{array}{r}
1.123 .347 .163 \text { francs } \\
502.064 .783 " \\
561.624 .190 " \\
87.059 .086 " \\
42.687 .635 " \\
\hline 2.318 .782 .859 "
\end{array}
$$

(1) Weber. D'après cet auteur, le chiffre relativement peu élevé des dépôts lans les banques allemandes s'expliquerait en partie par le grand nombre des dépôts faits aux caisses d'éparyne: ces dépôts représentent environ 188 fr. 20 par habitant. 
Les banques allemandes vont ehercher l'emploi de leurs capitaux sur un terrain plus rémunérateur.

Les Lombards (prèts sur titres) représentaicut pour l'ensemble des banques allemandes:

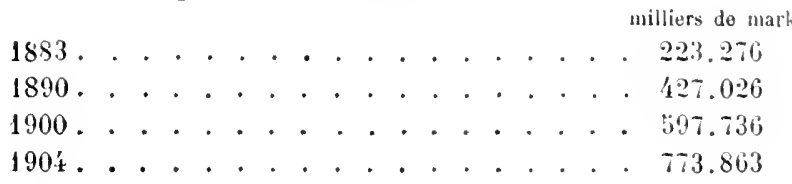

dont 447.880.000 marks arancés par les seules banques berlinoises: on voit l'important développement pris par cette branche classique de crédil.

Beaucoup plus considérables sont les crédits accordés au commerce el à lindustrí sous la forme de crédits en comptes courants; ils atteignaient:

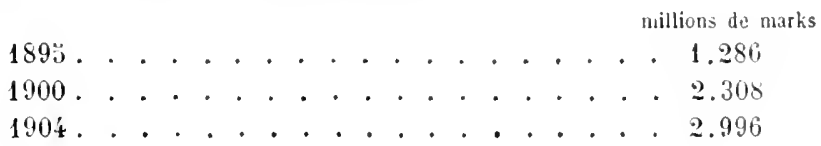

dont (1904) 383.6ł8.732 marks, à la Deutsche Bank

$\begin{array}{lll}328.538 .363 & - & \text { Dreshner Bank. } \\ 266.112 .009 & - & \text { Discontogesellschalt. } \\ 162.816 .321 & - & \text { Dirmstidter B. } \\ 236.366 .383 & - & \text { Schaathlaus. Bkv. }\end{array}$

Les économistes allemands se sont mainte fois élevés contre la pratique condammable deserédits paracceptation et des crédits en blanc: les premiers ont suivi une matrele progressive, passant pour l'ensemble des banques allemandes de 379 millions en $158 \%$, a 323 millions en 1890 , 1.294 millions en 1900 , et 1.399 millions de markis en 1904, dont 861 millions représentés par lés acceptations des banques berlinoises. Les banques constituent ainsi 
un fonds de roulement où leur crédit seul est en jeu et la clientèle spécule avec le crédit de ses banques.

Quant aux crédits en blanc (Blankocrediten), à découvert (ungedeckt), ils ont toujours joné dans les banques allemandes un rôle important: en 1900, plus de $310 / 0$ des crédits accordés étaient à découvert : cette proportion semble avoir diminué dans ces quatre dernières années (1), mais les banques n'indiquent pas toujours dans leurs bilans la distinction, essentielle pourtant, entre ces crédits en blanc et ceux que couvrent de sérieuses garanties réelles. Sans doute ces crédits ne sont accordés qu'après mùr examen $(2)$ et l'on ne saurait nier l'influence heureuse quent sur le développement économique du pays ce libéralismedes banques en matière de crédit; mais ceci ne va pas sans risques et sans dangers: nous le verrons lors de l'examen de la crise 1900-1901. Pour le moment, nous ne cherchons qu'à donner une idée de l'activité gé-

(1) Voir Bilans. - Deuxième partie.

(2) "Au lieu de se contenter l'un examen superficiel de la situation des personnes qui demandent un Blankocredit, plusieurs banques rhénanes exigent le bilan des dernières années; si quelque chose leur parait louche, si les chapitres leur semblent trop habilement composés pour les besoins de la canse, elles réclament les registres de comptabilité et les recristres copies de letires : puis sans trop se laisser toucler par la richesse de la maison, elles étudient l'importance, non pas absolue mais relative, des diverses parties des comptes. Elles n'oublient pas non plus de rechercher si l'industrje ou le commerce en jeu est susceptible de se développer ou est condamné à végéter. Enfin, à. l'aide de ces éments et par une étude attentive, elles arrivent à se faire une jdée exacte de la situation présente et en inème temps, pour ainsi dire, à premlre pied daus l'ilvenir. Comme elles imposent le plus souvent à leurs clients de leur remettre chaque année leurs bilans, elles surrent chacun et soit, conservent leur calme avec la certitude de ne point se tromper sur la situation réefle, soit se mettent à l’abri dans la mesure du possible "(Sayous, op. cit., p. 118). 
nérale des banques allemandes: nous voyons qu'elliss sont d’importantes banques de crélit ; "cest du banquier allemand plusque de tout autre qu’il est rai de dire qüil n'est plus un easisier doublé d’un trneur de live, mais qüil est un véritable marchand de réélit. C'est un commerçant, éest un intermédiaire entre les capitalistes désireux de placer leurs fonds et los entrepreneurs du commerce ou de lindustrie cherchant ì s'en procurer. Il est le trait d'union entre l'offre el la demande des capitaux $"(1)$.

"Les plus grandes maisons süntéressent au haut négoee et ì la grande industrie: les maisons moins importantes au moyen commerer et aux petites fabriques, les maisons de troisieme et de quatriome plan ont comme clientele les moyens patrons " (2).

Il nous reste à voir comment elles sont également des Sociétés financières, des banques d'alfaires, s'intéressant directement dans les entreprises industrielles et commerciales, non plus seulement par des owvertures de crédit, mais par toute une sírie d'operalions sur titres, imissions, participations et commandites. C'est lit, arons-nous dit, le trait caractéristique des banques allemandes: il a virement frappé tous ceux qui ont porté leur observation sur l’activité économique de l’Allemagne.

"La part prise par les banques a la constitution du capital des sociétés sous forme le commandites ou de participations, acrivail M. R. Monod dans un rapport ì la date du 14 juin lom1. les rend en quelque sorte solidaires

(1) G. Blondel, op cit., p. 18i.

(2) Sayous, op. cit.. J'. :\$5. 
les unes des autres. Pour un grand nombre d'établissements de crédit, la ruine des affaires industrielles ne constituerait pas seulement un ralentissement de leurs propres opérations financières, elle entraînerait une perte de capital énorme. On touche ici du doigt la hàte presque fébrile avec laquelle l'Allemagne a développé certaines branches de sa productivité et les inconvénients qui peuvent dans l'avenir en résulter pour elle. "

"Un trait distinctif de la méthode allemande, disaient MM. Fuller et Rowan dans un rapport à l'Institut of Bankers de Londres, est le lien étroit qui unit les entreprises industrielles et commerciales aux banques. Celles-ci ne se contentent pas de prendre dans les établissements de leur ordre des participations suffisantes pour exercer sur eux un puissant contrôle: elles suivent la même politique visà-vis des syndicats, comptoirs de mines, compagnies de chemins de fer, de tramways, etc...

"C'est un fait que le magnifique développement de leurs ressources nationales est dî dans une large mesure a l'initiative des banques qui, dans leurs bilans, font figurer ouvertement ces affaires et en discutent froidement les chances de succès: en Allemagne, le banquier est non seulement l'aide, il est le pionnier de leur commerce chez eux et au dehors. ")

L'ensemble des titres, participations et commandites possédés par les banques allemandes représentait:

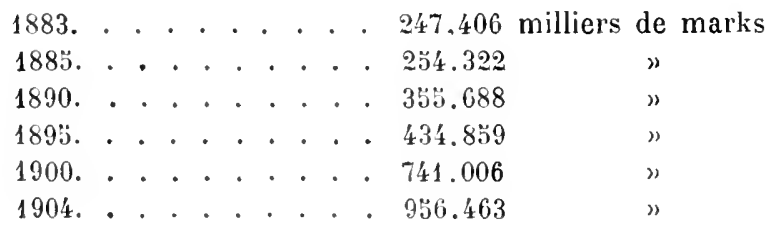


Pour les cing grands établissements, chefs de groupe, nous avons, pour 190', les ehiffres suivints (1):

\begin{tabular}{|c|c|c|c|c|}
\hline & & Titres & Participations & $\begin{array}{c}\text { (marks) } \\
\text { Commandites }\end{array}$ \\
\hline Deutsche Bank. . & & 82.293 .329 & $23 . .363 .873$ & 57.162 .498 \\
\hline Discontogesellschaft & & 73.78 .733 & 7อ̈.20:̈.639 & 17.730 .637 \\
\hline Dresdner Bank. . . & & 34.119 .382 & $4+.392 .113$ & $1+.939 .7 \%$ \\
\hline Darmstiidter Bank. & & $\ddot{6} 6.9 \div 8.732$ & $33.997 .01 \mathrm{t}$ & 28.279 .377 \\
\hline A. Schaaffh. Bkv. . & & $3+.58+.203$ & 20.426 .239 & 13.400 .183 \\
\hline
\end{tabular}

$A$ cette mime date, nous avions pour nos trois grandes Sociétés françaises de crédit:

Crédit Lyonnais, portefeuille, titres . . . . . 8.637.922 francs Société Générale

parlicipations industrielles et commerciales. . . . .

Comptoir d'Escompte, porlefenille, titres (com$46.473 .133-$ $2.030 .120-$ prenant également rentes, obligations et valturs diverses). . . . . . . . . . . 24.632.873 -

Nous aurons à ćtudier (voir B. Cause Economique) les différentes modalités que prend ce concours direct des banques allemandes dans l'activité des établissements industriels et commerciaux. Pour l'instant, le tableau suivant des émissions d'actions de banque et d'actions industrielles donnera une idée de l'aclivité des banques allemandes manifestée dans la création des valeurs mobilières (millions de marks):

(1) Pour l'analyse, roir II partie les monographies de ces dillérentes banques. 


\begin{tabular}{|c|c|c|c|c|c|}
\hline \multirow[b]{2}{*}{ Annees } & & \multicolumn{2}{|c|}{ Actions de banque } & \multicolumn{2}{|c|}{ Sociétés Industriel } \\
\hline & & $\begin{array}{c}\text { Capital } \\
\text { nominal. }\end{array}$ & Valeur au cours. & $\begin{array}{c}\text { Capital } \\
\text { nominal. }\end{array}$ & Valeur au $\mathrm{c}$ \\
\hline 1894. & . & . $\quad 31,6$ & 36,2 & 60,3 & 79,0 \\
\hline 1895. & . & . 113,0 & 143,1 & 161,2 & 223.2 \\
\hline 1896. & . & . $\quad 157,8$ & 213,4 & 245,3 & 333,9 \\
\hline 1897. & . & 173,1 & 265,7 & 190,9 & 318,2 \\
\hline 1898. & . & $2 \tau 3,3$ & 372,8 & 310,2 & 320,6 \\
\hline 1899. & . & 211,6 & $276, \ddot{3}$ & $515 ั, 9$ & 861,4 \\
\hline 1900. & . & 138,0 & 174,5 & 297,4 & 461,1 \\
\hline 1901. & . & 30,6 & 36,2 & 116,0 & 160, \\
\hline 1902 & . . & 81,4 & 114,3 & 164,2 & 181, \\
\hline
\end{tabular}

Les banques allemandes nous apparaissent done bien comme étant à la fois des banquesdedépòl et de crédit et des banques d'affaires: elles combinent ces fonctions dans une proportion variable mais toujours plus que suffisante pour donner à ce fait une valeur générale ot caractéristique.

Ajoutons enfin que le total des bilans des banques allemandes n'a cessé de s'accroîlre régulièrement pendant ces 20 dernières années (Deutsche OEkonomist, 29 juillet 1903̈).

1883. - 1.961,7 millions de marks

\begin{tabular}{|c|c|c|c|c|}
\hline 1884. & $2.122,6$ & $D$ & $+160,9$ & de marks \\
\hline 1885. & 2. 229,9 & $"$ & $+106,4$ & $"$ \\
\hline 1886. & $2.283,4$ & $»$ & $+54,3$ & ) \\
\hline 1887. & 2. 338,8 & $"$ & $+55,4$ & ") \\
\hline 1888. & 2.529,8 & " & $+191,0$ & $"$ \\
\hline 1889. & $3.156,2$ & $"$ & $+626,4$ & $"$ \\
\hline 1890. & $3.149,9$ & 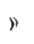 & $-\quad 6,3$ & " \\
\hline 1891. & $3.10 \mathbf{4}, 0$ & $"$ & - $4 \ddot{5}, 9$ & $"$ \\
\hline 1892. & $3.15+5$ & $n$ & $+47,3$ & $n$ \\
\hline 1893. & $3.167,3$ & $n$ & $+\quad 13,8$ & ") \\
\hline 1894. & $3.593,6$ & $n$ & $+426,2$ & $"$ \\
\hline 1895. & $3.933,2$ & ") & $+339, \pi$ & $"$ \\
\hline 1896. & $4.214,7$ & $"$ & $+281,5$ & $n$ \\
\hline 1897. & $4.718,0$ & 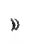 & $+503,3$ & $"$ \\
\hline 1898. & $5.673,3$ & $"$ & $+955,5$ & $D$ \\
\hline 1899. & $6.466,4$ & $\eta$ & $+792,9$ & . \\
\hline
\end{tabular}


1900. - 6.9.8, millions de marks $+491,6$ millions de marks

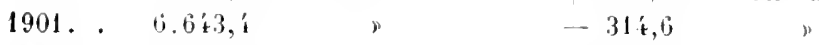

1902. $7.093,1+4.7,7$,

1903. $7.550 .6+458.500$

1906. $8.460,3$ +

Dansce total les banques herlinoises représentent en 1590 un areroissement proportionnel de $8100: 0$ 1901, de 丂ั9 00 .

Quelles sont maintenant. au point de vue de la concentration, les conséquences de cette façon de concevoir et de pratiquer la banque, l’intluence exercée par la technique mème du commerce de banque en Allemagne?

"Dans la banque, dit M. Bourguin, un mouvement d'intégration se poursuit parallèement à celui de la concentration "(1). Nul doute quen Allemagne, le mouvement d’intégration particulierement farorisé par les circonstances historigues ct le aractère germanique luimème, n’ait par la singulièrement aidé à la concentration.

"Les banques allemandes, at-t-on dit, mènent de front les opérations les plus direrses arec autant d’énergie que si chacune d'entre elles formait l'oljet unique de leur activité : elles prolitent des opérations d'une certaine catégorie pour lévelopper arec une plus grande intensité les opérations d'une certaine autre."

a) L'activité de la Banque ainsi élargie el renforcée, décuplée en quelque surte. va d'abnel nécessiter un renforcement de ses moyns dexploitation propres, d'oir ces augmentations suecesseres du rapitul que deja nous avons montrées; premier élément de concentration.

(1) Bourguin, pp. cit., p. 1'0. 
"Les banques allemandes, écrivait en 1898 M. Raffalovich, semblent aujourd'hui en proie à une véritable manie de grossir leur capital, ce qui tient en partie à ce qu'elles cumulent des fonctions qui sont séparées dans d'autres pays" (1).

Ce n'est point seulement, en effet, le souci ou l'obligation de toujours maintenir une certaine proportion entre leurs ressources propres et leurs engagements envers les tiers, qui a dicté aux banques allemandes ces augmentations de leur capital-actions: on verra que ce n'est point là qu'il faut en rechercher la raison première, si l'on se reporte aux tableaux que nous avons donnés (p. 54) et qui indiquent à còté du chiffre des dépôts et des comptes créditeurs le montant des ressources propres: c'est au contraire uniquement ce souci qui paraît avoir inspiré les élévations du capital de nos grandes sociétés françaises de crédit :

Crédit Lyonnais :

\begin{tabular}{|c|c|c|c|c|c|c|}
\hline & & illion & & & & millions \\
\hline 1873. & capital & 50 & Ressources & provenant des & tiers & 179 \\
\hline 1875 & $"$ & 73 & $"$ & 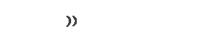 & $"$ & 214 \\
\hline 1879 & $"$ & 100 & $"$ & $"$ & ” & 280 \\
\hline 1881 & $"$ & 200 & $"$ & $"$ & $n$ & 383 \\
\hline 1900 & " & 230 & $"$ & 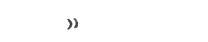 & $n$ & 1.000 \\
\hline
\end{tabular}

Comptoir National d'Escompte:

\begin{tabular}{|c|c|c|c|c|c|c|}
\hline 1889 & capital & 40 & Ress & ren & tiers & 140 \\
\hline 1890 & $n$ & 80 & " & " & $"$ & 211 \\
\hline 1892 & $"$ & 75 & " & ") & $"$ & 201 \\
\hline 1895 & $"$ & 100 & $"$ & ") & $"$ & 303 \\
\hline 1900 & $n$ & 150 & » & " & 》 & 472 \\
\hline
\end{tabular}

(1) Raffalovich, Marché financier, 1898-1899. 
Société Giénérale:

1878. capital 120 Ressources provenant des tiers

millions

1899 . " 180 " "

$1904 . \quad 200$

568

722

Mais il faut voir yue les dépòts jouent dans ces élablissements un róle prépondérant: éest eux - et eux seuls peut-on dire, si l'on considere les ressources propres comme étant surtout un fonds de yarandie - qui forment la base des opérations courantes de ces banques, la source normale et principale de leur's prolits:

"Nous ne négligeons pas les affaires financières, disait le rapport du Crédit Lyonuais en 1880 ; nous les accueillons volontiers quand les circonstances nous les présentent. Mais nous ne voulons pas dépendre d'elles; nous roulons avoir notre revenu innuel assuré par les bénćlices ordinaires de banque. ")

Le rapport de 190't tenait le mème langage . "Les bases de la gestion du Crédit Lyonnais sont toujours les mèmes: eonserver aux disponibilités leur abondance atin de donner une base solide au crédit de liontreprise. n'almettre dans laatif dautre immobilisation que celle résultanl des immeubles sociaux, contrólerseverement les risques et las diviser le plus possible en ritant les pros engagenents. n

Tout au contrare, les intreventions plus nombreuses et plus hardies des banques allemandes dans l’émission des valeurs industrielles et dins la speculation a cu pour conséquence naturelle diattribuer amx ressourees propres un robeplus important. En l:to f par axemple, dans les han-

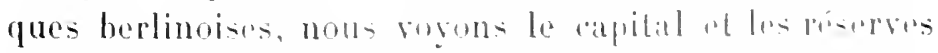

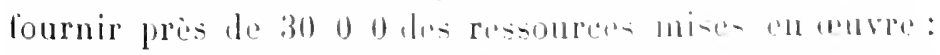
pour nos trois grandes socićlés de crédil, celle proportion 
était à peine de $180 / 0$ : dans les banques anglaises, capital et réserves représentent 8 ou 900 des moyens de crédit (1).

De plus, cette situation qui oblige les banques allemandes à rechercher les affaires à gros bénéfices, ne va pas sans des risques plus élevés auxquels échappent plus facilement les grandes banques. Quand en effet les dépôts atteignent dans une banque un chiffre considérable, les probabilités de remboursement ne s'ćcartent guère d'une certaine moyenne. Il devient alors possible de consacrer sans imprudence une partie des ressources disponibles à des opérations de plus longne haleine, moins facilement liquidables, mais à rendement plus élevé. Cet avantage des grandes banques n'a pas peu contribué à précipiter le mouvement de concentration.

b) Quand les grandes banques développèrent leurs opérations de banque proprement dites, - poussées par la nécessité qui s’imposait à elles chaque jour davantage, d'emprunter pour pouvoir prêter, -- clles purent, assurées d'autre parl de larges bénéfices, faire sentir plus âprement leur concurrence aux petils banquiers, en offrant à la clientèle des conditions spéciales, plus a vantageuses; cette nécessité les conduisit également à multiplier le nombre des caisses de dépôts ; il n'est pas douteux en effet que "le système d'exploitation par agences ne soit la condition indispensable pour développer presque à l'infini les dépôts (2) ». Entrées lans cette voie, où les poussait encore l'influence particulière de ces lois de finances et du Börsengesetz que nous amrons à étudier, elles se sont attachées

(1) V. Aupetit, Vie Financière. Revue Economique Internationale, mai-juin 1904 .

(2) Saurel, op. cit, p. 77. 


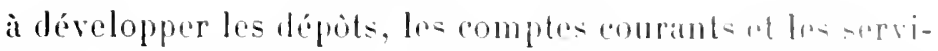
ces de Girokontos qui ont pris an Mllomagne unr planen considerable: nous narons pas besoin de lomontererom. ment ces deux dernieres catégories dialliares ne punvent prospérer que si on les pratique sur une gramde échllo: la compensation na de but que lorsqu'un grand nombre de maisons y prennent part.

C’est pour élargeir leur cercle de compensation, coneentrer les opérations de paiement, ne régler pur des soldes, réaliser ainsi de notables économies de frais généraux, timbre, commissions, que nous ropons les grandes banquesétendre leur réseáu de suceursales el de bureanx, sallier entre elles. Il faul ágalement noter daus cet ordre d’idées que l'acès direct au lieu de compensalion. cestà-dire à la Reichsbank, axige grion ymaintionne un solde de compensation (Girosaldo) trin considérable et seuls de très grands établissements peurent satisfaire à cette exigence (1).

Le mouvement d'expansion causé par le développement des affaires régulieres do banque est ainsi venu doubler celui qu’avait déjà nécessilé, pour les yrands chtablisosments, intéressés aux entreprises industrielles, to hesoin

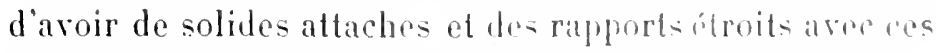
entreprises, sur les linus mimpis de four activilí. comme aussi pour pouroir plut facilement anouler alux guichets de leurs succursales on des banques allieres, les innombrables titres industriels ámis fall eux. Yous don-

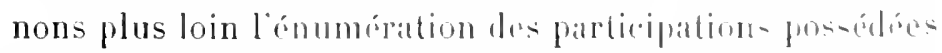

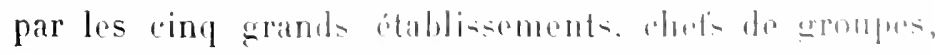

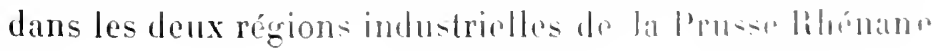

(1) Fleischhammer, art. cili. 
et de la Silécie. Jiçons seulement en passant, qu'ils y possèdent 21 participations et 70 filiales représentant un capital-actions de \$\$2.140.800 marks.

c) Enfin nous avons noté la progression remarquable suivie dans les grandes banques allemandes par les crédits d'acceptations qui l'eprésentent " un capital, que ni les actionnaires ni les déposants n’ont fourni, qui n'est renfermé dans aucune caisse ni dans aucun portefeuille (1)", capital créé de toutes pièces, peut-on dire, par les banques elles-mèmes. et qui repose uniquement sur le crédit. Or le crédit d'une lıanque est une chose essentiellement subjective, il est fait de la confiance que met en elle un plus ou moins grand nombre de personnes: " la publication des bilans qui sert aux luanques d'annonces perpétueltes» (2), l'étendue de leur's relations sans cesse affirmée, les innombrables moyens qu'elles emploient pour frapper les yeux et l'imagination du public, établissent et fomt comaitre le crédit des grandes banques. On roit comment les grands établissements berlinois savent en tirer un large parti.

Sans doute, un grand nombre de ces éléments techniques partieulierement favorables aux grands établissements et facteurs de leur supríriorité sur les petites institutions, ne sont point spéciaux aux banques allemandes. Une phus lurye compensation de risques, par l'effet de la loi des grinds nombres, une action plus étendue sur le public, grâce ì leurs agences el correspondants, gràce aussi à la puissance le crédit attachéc à leur signature, une utilisation toujours active de leurs capitaux par leur établis-

(1) Saurel, op. cit., p. 8:?

(2) IV. Bagehot, op. cil., 1. 257. 
sement sur un gromel nombre de plares atux conditions

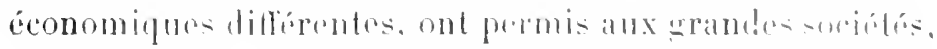

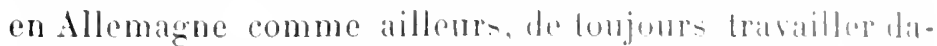
vantage, de travailler à moindres frabs ot avec plus du

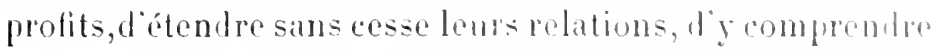
à la fois capitalistes, commereanls, industriels, communes et Etats mime, daceroitro les branclies de leur activilé et le chillre de leurs aflabres. les dillérente aspects do la supóriorité des grandes hampuessur les pretiles-canse générale de la concentration - ont étí maintes fois oxposés; aussi nous natrons point voulu en reprendrelanalyse el nous y attarder: nous aroms surtoul cherche indiquer dans qualle mesure, cofail particulier à l'Mlle-

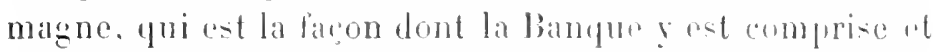
prabiquée, a favorisé lo dévoloppemont des garaldes banques et leur a assulé une nomralle supropiopile daus la lutte de la concurrence, an leur ollrant de multiples moyens dexereer lemr activité, - al somvent sur mu ter-

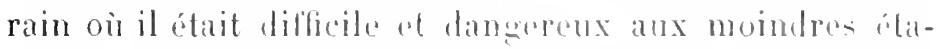

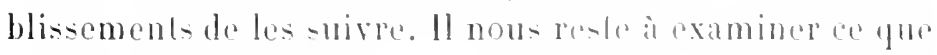
nous avons appelé las cauce icomomique du mourement de concentration.

\section{I3. - La cause ínonomique}

11 est assez évidont quo cést le dereloppement iromo.

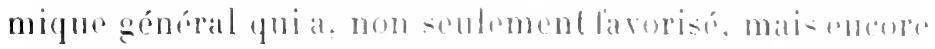

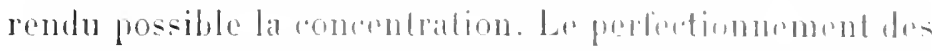

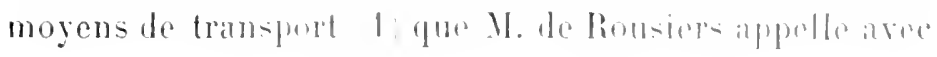

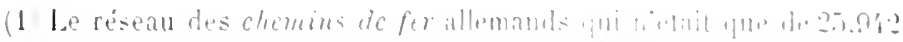

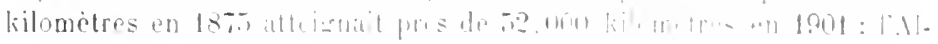


juste raison "les pères de la concentration commerciale ", l'extension des communications postales, télégraphiques et téléphoniques (1) ont permis de nouer des relations étroiles entre les clients les plus reculés de la province et les srandes banques de la capitale.

D’autre part, l'avènement des grandes sociétés industrielles modernes a nécessité la eréation d'organismes de erédit et d'échange que ne demandaient point l'industrie à domicile ou le petit commerce local. En un mot, pour que la grande banque, comme la grande exploitation, prît naissance " il fallait qu'un système de relations développées, des Etats d'étendue plus considérable et une politique commereiale et coloniale heureuse eussent entrainé la formation de grands marchís facilement accessibles, que les bornes mises à l'intérieur aux relations du commerce et à la coneurrence eussent disparu... il fallait ensuite que le commerce ent produil une bourgeoisie pleine d'audace et de confiance en soi, eùt développé un esprit débordant d"initiative et dentreprise commerciale... il fal-

lemagne possèle en outre environ 15.000 kilomètres de voies fluviales navigables.

(1) Le réseau ţlegraphique allemand représentait:

1880. . . . 70.826,540 kilomètres

$1890 . .2103 .308,910-$

1900... 128.315,000 - (non compris 37,715 kil. de lignes de chemins de fer).

Le développement en kilomètres du réseau de fils télèphoniques représentait :

$1890 . .2 .11$ 1.260 kilomètres

1900. . . . 750.291 -

1903. . . 1.632.190 - (pour la France, seulement 637.579 kil.)

(Statistiques du Bureau International des administrations télégraphiques à Berne). 


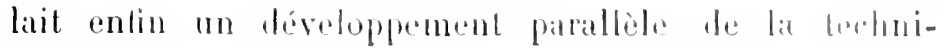
que $n(1) \ldots$

Mais ést moins de cel ensemble de condilions grimpirles que nous roulous parler ici que diunre cause phu parte ticuliere à l'Allemagne, tout a lait propore a explipurer la rapidité du monvement de concentration el sur laguelle arec me largour de wes el un sens eritique asce rares chez de purs lommes datiares, insistatent virement les binquiers que noms arons eu la bomme forlum. te pouvoir approcher et consulter.

"Prenczgarde, nous disail a Franefort. Le direwteur de la suceursale diun grand iblulissement berlinois: guand vous interrogerezdes banquires sur la concentration el sur ses causes, on rous parlera smetout de la technigue de la compensation, des lois sur le limbre el sur la bunrse. Ce ne sont pas là les raies causes de lat concentration. A mon aris, ces lois nont lait que sontenir une fundance gincrale qui se manileste dans la banque comme dams fontes las antres branches du commerer at de lindustrie : celar est pourlant bien sensible chez nous : éet la throrioldes rasers communiquanls. n

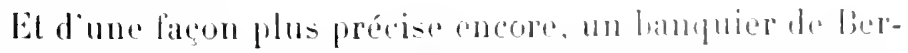

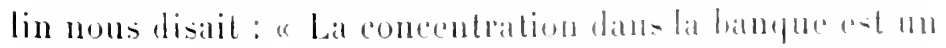

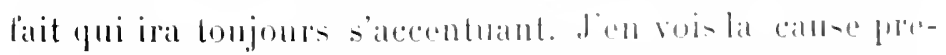

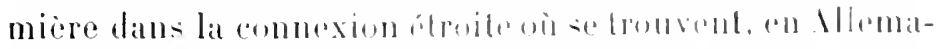

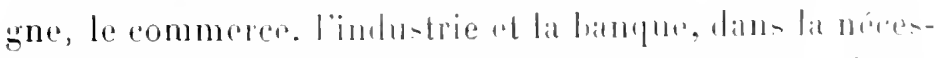

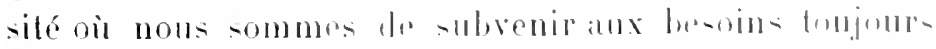

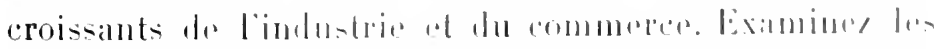

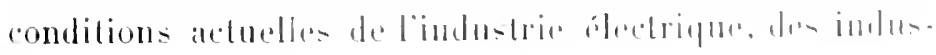

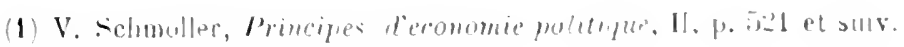


tries du for ou du charbon par exemple; il est évident que ce ne sont plus les mêmesqu'au moment où nous avons aidé ces industries à se mettre en marche; clles ont aujourd'hui des besoins de capitaux et de crédit, auxquels ne peuvent plus suffire les banques moyennes dont les ressources, mème fortifies par des dépôts, sont devenues insuffisantes: la grande industrie nécessite de grandes banques aux capitaux considérables et disponibles, "

("'est en effet, suivant nous, dans cette connexion étroite, dans cette union intime des banques allemandes et de l'industrie et du commerce, qui fait des grands établissements des sociétés linancières aulant que des intermédiaires d'échange et de crédit, qu'il faut chercher l'explication de l'intensité et de la rapidité du mouvement de concentration dans le trafic de banque.

Sans doute, d'une façon générale, "l'union de la banque et de l'industrie est imposéc par le développement économique: d'un côté, les entreprises industrielles que les capitaux accumulés individuellement ne suflisent plus ì etablir, sont dépendantes des banques pour les capitaux nécessaires ì leur fonctionnement; de l'autre côté, les banques concentrant les capitaux que n’absorbent pas les emprunts d'Elat et qui ne trouvent plus d'emploi dans la petile industrie, sont obligés pour les faire fructifier de les mettre à la disposition res grandes sociétés industrielles " 1. Et Schaeflle avait montré déjà comment " nous sommes arrivés à ce poinl du développement économique, oì la crande industrie doit en grande partic compléter son capital par la réunion des petits capitaux fournis par les organes fronomiques particuliers qui ont pour fonc-

(1) P. Lafargue, Les Trusts américains, p. 103. 
tion spéciale liamettre les actions industriclles: le driveloppenent des banyues deentrepri-es est parallele a celui de la grande industrien.

Mais ce fait présente en Allemagne un caractire particulièrement original en ce sens que "res hanques l'entreprises " sont en mème temps les banpues ordinares et quelles travaillìrent des le dibut, à la constitution des dillérentes industries; le rapport est ainsi plus étroit, l’intluence réciproque plus puissante.

"Les banques chez nous, acril M. Blondel, assurement ne refusent pas leur concours à l'industrie, mais ce qu'elles ne pratiquent pas, cest une réritalile coopération, un consortium, comme disent les Américains, entre le producteur, le commereant et le banquier. Cette entente est au contraire un des éléments de succès de nos voisins. Ce sont les banques allemandes qui ont arancé il y a vingteinq ans, les sommes nécessaires à la transformation et à la construction des usines. Depuis lors, ce sont elles qui ont permis à ces átabliesements de so soutenir. de traverser les époques do crise, le so maintenir a la hauleur du progris: clles ont contribur pour mon large prart à l'essor économique de l'Allemagne (1)."

(1) Blondel, op. cit., p. 482. "Les banqulers, dıt .l. Sayous, parlant de la transformation économique de l'dlemaene, furent plus que des intermédiaires ordinaires entre le capital te travail; ils dirigerent ou soutinrent le mourement avec une extrime fermete. Ils transformirent les possibilités et les puissances pn des réalitis et prèterent personnel. lement an commerce et il limdustrie l'appui qui leur fatait micessaire."

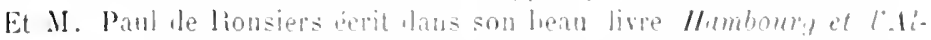
lema!ne contemporate, J. 2:31: a le comprents mienx apris latroil

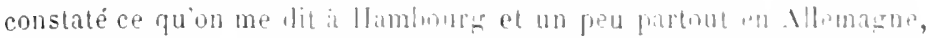
du rile très fecond des bampes tans les entreprise industriplles et commerciales : Ici l'argeut est comtiant. Les hanyue mus soutiemnent

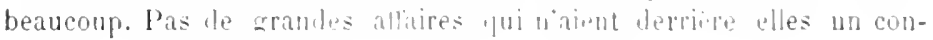


"Gràce à l'appui et au concours, que la haute finance lui a prètés, l'industrie allemande a pris en peu de temps un développement gigantesque, - écrivait la Chronique industrielle, maritime el coloniale du 6 janvier 1905. Il n'est point douteux que sans cette alliance, sans cette union des forees industrielles et des forces financières, l'Empire n'eût point réalisé ces merveilles dont nous avons été les témoins. "

Il est très visible en effet, qu'à part Berlin, qui s'est trouvée comme capitale de l'Empire dans une situation tout à fait particulière, la banque s'est, en Allemagne, beaucoup plus développée à l'ouest de l'Elbe qu'à l'Est de ce fleuve et cela d'une façon parallèle à la répartition de l'industrie ot de la richesse en capital du pays.

La solidarité qui unit les banques aux entreprises industriclles et commereiales a revêtu des formes variées: les Banques ont parfois créé de toutes pièces des usines, surtout au début du développement économique de l'Allemagne: l'A. Schaalfausens eher Bankverein fit ainsi construire pour son propre compte des fabriques dans la province rhénane. Dès 18. II (l'A. Schaatfhausens'cher a été fondé en 1848), son compte "participations dans les sociétès industrulles " atteint 434.706 thalers: if s'intéresse en ISöl à la Hörder Berswerks und Hüttenverein, en 18.92 à la llörder Bergwerlisverein, à la köhlnische Baumwolls-Spinneroi und Weberei. Dans la seule année 18506 , la Darmstädter Bank participe à la fondation de la Wolls-

sortium de banques. Dans ce pays on a fait pour ainsi dire l'éducation du capital ", tels sont les témoignages concordants que je recuejlle de toutes parts chez les armateurs, les industriels, les négociants, les consuls et les économistes; inutile d'ajouter que les banquiers n'y contredisent pas." 
manafactur de Manmheim (cappital 100.000 Morins), la la

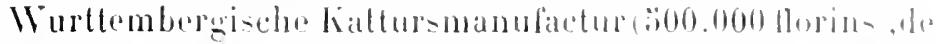

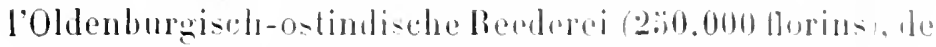
la fabrique de lainages el peignés de Markli-sa l:300 .000 1lu-

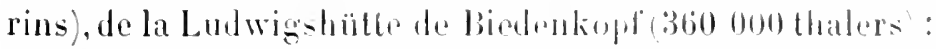
elle participe avec le concours do la Hiltedent-cho CreditBank à la transformation de la Maschinenfabrik und Eisengiesserei de Darmstadt en me sociéle pal arlions au capital de 200.000 norins, at la tramsformation de l'lleilbronner Maschinembaugerellschalt.

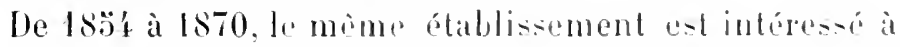
l'émission des atctions el oblimations de 33 socirtís le clurmins de fer ; la liscontoge-ellsthatt aux émissions de 8 sociétés du même getenre.

Plus souvent, en ves dernieres anmés, les banques ont commandité les élablissements imdustriels et commerciaux; elles ont transforme les entreprises privés dija existantes en sociétris par autions ou ménage les angmentations de capital de cos sociotis, sontrmant le cours des actions, rachelant souvent pour leur propre comple les titres quelles inscrivent dans lemrs bilans sous le nom dr

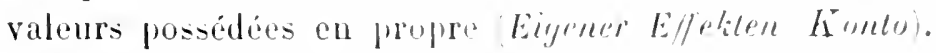

Nous ne pourous songer it donner ici l'entumbation complète et détaillée des ditrirenten ot multiplen antrepri-

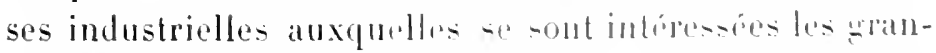
des banques (l) : si lon se reporte an tableam que mous

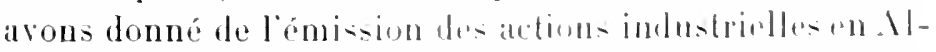
lemagne, on verra ïimportance de lartivitédrplogédens

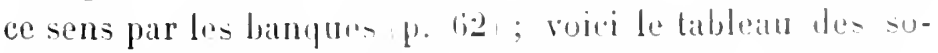
ciétés par actions erééc en . Mlemagne depuis 1870.01 ce-

I V. 20 partie, le detail du comple cunsortial des fronds chatissements. 
lui du Konsortial konto des grandes banques: mieux que tous les développements, ils montreront les participations directes prises par elles dans les sociétés industrielles. Donnons seulement, comme détail, la liste des différentes émissions ou fondations auxquelles a participé la Deutsche Bank en ces dernières années :

Actions ou obligations de chemins de fer . . . 25

Entreprises de transport et de navigation. . . . 27

Brasseries . . . . . . . . . . . 15

Mines et industries métallurgiques. . . . . . . 54

Industries textiles. . . . . . . . . . . . 24

électriques. . . . . . . . 14

chimiques. . . . . . . . . 17

Construction de machines . . . . . . . . 32

Briqueteries .. . . . . . . . . . . 5

Entreprises diverses. . . . . . . . . . . . . 40

258

On ne comptait pas moins en Allemagne, fin 1904, de \$.482 sociétés par actions représentant un capital de 815.270 millions de marks.

Il a été fondé en Allemagne de 1870 à 1904:

$\begin{array}{cccc}\text { Années } & & \text { millions de marks } \\ 1871 . & 207 \text { sociétés par actions au capital de } & 758,76 \\ 1872 . & 479 & - & 1.477,73 \\ 1873 . & 242 & - & 544,18 \\ 1874 . & 90 & - & 103,92 \\ 1875 . & 55 & - & 45,56 \\ 1876 . & 42 & - & 18,18 \\ 1877 . & 44 & - & 43,42 \\ 1878 . & 42 & - & 13,25 \\ 1879 . & 45 & - & 57,14 \\ 1880 . & 97 & - & 91,59 \\ 1881 . & 111 & - & 199,24 \\ 1882 . & 94 & - & 56,10 \\ 1883 . & 192 & - & 176,03\end{array}$




\begin{tabular}{|c|c|c|c|}
\hline Années & & & millions de 11 \\
\hline $188^{\prime}$ & $1 ; 33$ & sociétés pas actions au capital de & $111,2 \dot{2}$ \\
\hline 1885. & 70 & - & $53,+7$ \\
\hline 1886. & 113 & - & 103,94 \\
\hline 1887. & 168 & - & 128,11 \\
\hline 1888. & 181 & - & 193,68 \\
\hline 1889. & 360 & - & 402,$5 ;$ \\
\hline 1890. & 236 & - & 270,99 \\
\hline 1891. & 160 & - & 90,24 \\
\hline 1892. & 127 & - & 79,82 \\
\hline 1893. & $9 \ddot{3}$ & - & 77,26 \\
\hline $189^{\prime}$. & 92 & - & 88,26 \\
\hline 1895. & 161 & - & 250,86 \\
\hline 1896. & 182 & - & 268,58 \\
\hline 1897. & $20 \%$ & - & $3 \varsigma 0, \mathfrak{t} 7$ \\
\hline 1898. & 329 & - & 663,152 \\
\hline 1899. & $36 t$ & 一 & $5 \div \div, 39$ \\
\hline 1900. & 261 & - & $340,+6$ \\
\hline 1901. & 108 & - & $138,2 \%$ \\
\hline 1902. & 87 & - & 118,43 \\
\hline 1903. & 84 & - & $300,0 x^{\prime}$ \\
\hline $190 x$. & $10 \dot{x}$ & - & $1\{0,6$ \\
\hline & $48 \div$ & & $81 \%, 270$ \\
\hline
\end{tabular}

Or, de ce capital total, les grandes banques allemandes possèdent une part considérable comme te montre le tableau suivant:

\begin{tabular}{|c|c|c|c|}
\hline Fin 1904 & $\begin{array}{l}\text { Valeurs prossédées } \\
\text { en propre }\end{array}$ & 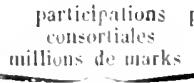 & $\begin{array}{r}\begin{array}{r}\text { participal.durah } \\
\text { commandite } \\
\end{array} \\
\end{array}$ \\
\hline Bank.. . . & 82.2 & 23.3 & 37.1 \\
\hline resellschaft. . & 73.2 & - & ii.:- \\
\hline Bank... . & $\ddot{z i} .1$ & 108.2 & - \\
\hline fThaus. Banky & $3 t .: 3$ & 33.8 & - \\
\hline ter Bank. . & :iti.9 & 34.8 & 23.1 \\
\hline & 3102.99 & 200.3 & 1118 \\
\hline
\end{tabular}

¿9i,li millions de marlis

Sans donte, de ces 7996,6 millions de marks, il fant dé- 
duire les parts prises par lés grandes banques dans l’émission des emprunts communaux ou d'Etats, ainsi que les participations prises dans les entreprises industrielles et commerciales étrangères; il reste cependant un total de capitaux ind ustriels et commerciaux allemands, concentrés dans les banques, plus que suffisant pour établir, d'une façon globale, l'étroitesse des rapports qui unissent en Allemagne l'industrie, le commerce et la banque.

Ils sont par exemple, particulièrement sensibles dans l'industrie électrique que le rapport de la chambre de commerce de Stuttgart de 1901 nous montre justement "en train de conquérir la première place sur le marché du monde ". Ces entreprises ont trouvé dans les banques leurs plus précieux auxiliaires; aux 7 principaux groupes électriques correspondent en Allemagne, 7 groupes de banques, chargées de financer l'affaire et possédant en portefeuille les actions d'entreprises anciennes achetées en vue de transformation ou celles des entreprises déjà transformées :

\section{Grompe Siemens et Halske.}
1. Deulsche Bank.
7. S. Bleichröder.
2. Darmstädter Banti.
8. Delbrïck, Leo et Cie.
3. Berliner Handelsgesellschaft.
9. Jacob S. H. Stern.
4. Discontongesellschaft. 10. L. Speyer Ellisen.
5. Drestner Barki.
11. Bergisch Märkische Bank.
6. Mitteideutsche Credit bank.

\section{Allgemeine Elelitricititsgesellschaft.}

1. Berliner Llandelsgesellschaft.

2. Deutsche Bank.

3. National Bank.

4. Delbrïck Leo et Cie.

ॐ. Hardy et Cie.
6. Sulzbach.

7. E. lleimann.

8. Rheinische Discontogesellschaft. 


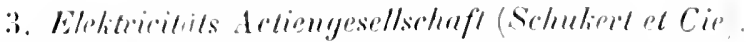

1. Sïideutsiche lisiconloy.

2. Anton kiolin.

3. Kommerz n. Diseonto $B$.

4. de lleydl-liersen.

3. E. Ladenburg.

\section{Cninn Elelituirititsgesellschaft.}

1. Discontogesellschaft.

4. A. Scharflhansens'rher Bhe.

2. Dresiner Bank.

i. S. Bleichrioler.

3. Darmstädler Bank.
6. J. Drevfus et Cie.

7. Bayerische lereinsbank.

8. Bayerische Hypoteken $1 \mathrm{We}$ chsel bank.

i. Ilelios Elelitricititsgesellschaft.

1. I L. Eltzlacher.

2. J. II. Stein.

3. Oppenheim el Cie.

4. Deutshe Gimossemschafts b.

5 . Berliner Bank.

6. Elehtriritits Actiengesellschaft (It.Lahmeyer et (ie).

1. Dresdner Bank.

2. Darmsliadter Bank.

3. Cironelius et Cie.

4. Oberrheinische li.
6. (. schlesinger el cie.

7. Meutsche Eireckten und Weclselbank.

\&. Niederrheinische Credit Anstalt.

7. A ctiengesellschaft Eletiricititswerlie (0. L. Summer "Cie).

1. Diesdner Bank.

2. Credit Anstalt für Industrie und llandel.

Ciest la meme politique quiont suivir sur méchello plus ou moins grande, les bangues allemandes vis-it-vis

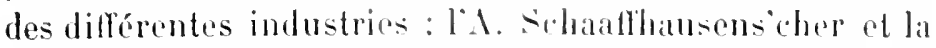
Dresdner Bank s̈intriressunt plus particuliorement aux

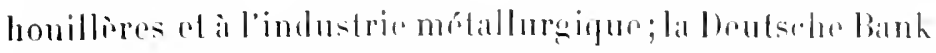
aux industries électridurs: la llisoonto aux entreprisu de transport.

Cette union siest alfirmée en cesdernicrstemps d'une 
façon plus précise; par des échanges d'administrateurs, les industriels devenus gros actionnaires des banques, sont entrés au conseil des grands établissements et ceux-ci ont délégué de leurs membres au conseil des entreprises qu'elles commanditent :

Nous trouvons ainsi (1904) :

Dans le conseil de la Darmstädter Bank . . . . . 4 industriels

$\begin{array}{lllll}\text { - } & \text { Berliner llandelsgesellschaft. } & \text { 12 } & - \\ \text { - } & \text { Deutsche Bank . . . . . . } & \text {. } & 4 & - \\ \text { - } & \text { Discontogesellschaft . . . . . } & 5 & - \\ \text { - } & \text { Dresdner Bank . . . . . . . } & \text { 5 } & -\end{array}$

Celles-ci ont, d'autre part, des administrateurs délégués dans les différentes entreprises suivantes:

\begin{tabular}{|c|c|c|c|c|c|}
\hline & & $\begin{array}{l}\text { Mines et } \\
\text { houillères }\end{array}$ & $\begin{array}{l}\text { Fabriques de } \\
\text { machines }\end{array}$ & $\begin{array}{l}\text { Industries } \\
\text { chimiques }\end{array}$ & $\begin{array}{l}\text { Industries } \\
\text { électriques }\end{array}$ \\
\hline Deutsche Bank. & . & . 9 & 4 & 2 & 14 \\
\hline Dresdner. . . & . & 7 & 4 & 1 & 6 \\
\hline Darmstädter. . & . & 8 & 7 & 3 & 8 \\
\hline Disconto.... & . & 10 & 6 & $n$ & 6 \\
\hline Berliner Handels & . & 10 & 3 & $"$ & 9 \\
\hline A. Schaaffh. Bkv. & & $2 \%$ & 9 & 2 & 3 \\
\hline
\end{tabular}

On comprend mieux maintenant ce que voulait dire le banquier allemand en parlant de la théorie de vases communiquants et comment l'action du mouvement de concentration qui se manifeste dans l'industrie et le commerce a pu, en Allemagne, inlluer plus fortement sur le mouvement de concentration dans la banque. La réciproque paraît également vraie d'ailleur's; nous ne donnerons pas ici un tableau des progrès de la concentration industrielle et commerciale; nous tenons le fait pour acquis. Voici simplement quelques chiffres propres à fixer les idées, que nous empruntons au précieux livre de M. Bourguin et qui éta- 


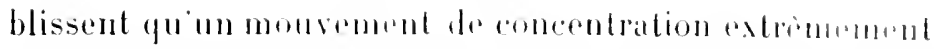

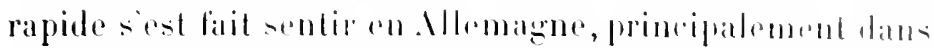
les industries lextiles. himigues, les mines. la constrnce tion des machines, la minolerie, le travail des mitamx ot lindustrie du batiment (1):

Industrie. - Nombre ales exploitations en 1852 et $189 \%$.

\begin{tabular}{|c|c|c|c|c|c|}
\hline \multirow[b]{2}{*}{ Cat gories } & \multicolumn{2}{|l|}{$1 \times 2 \geq$} & \multicolumn{2}{|c|}{$1 \times 9.6$} & \multirow{2}{*}{ 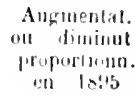 } \\
\hline & Nomlire & propirt. & Nombre & propert. & \\
\hline $\begin{array}{l}\text { Pelitesexploitations } \\
\text { (1 i : } \text { i personnes) }\end{array}$ & & & & & \\
\hline $\begin{array}{l}\text { Alleinbetriebe sans } \\
\text { moteur). } \\
\text { Gehrilfenbelriebe }\end{array}$ & 1.430 .463 & $"$ & $1.237 .3+9$ & $"$ & " \\
\hline (avec moteur. & 活.3.392 & $9 \% .8$ & 732.223 & 92.6 & 8.6 \\
\hline Tolal. . . & $2.175 .80 \%$ & & 1.989 .872 & & \\
\hline $\begin{array}{l}\text { Moyennes exploita- } \\
\text { lions (6 à } 50 \text { per- } \\
\text { somnes) }\end{array}$ & & & & & \\
\hline Gonnes exploila- & 8.3 .001 & 3.8 & 139.569 & 6.5 & $+6 t .1$ \\
\hline $\begin{array}{l}\text { lions }+ \text { de } \ddot{0} 0 \\
\text { personnes. }\end{array}$ & 9.41 & $11 . \dot{t}$ & $17.9+1$ & 0.9 & $\div 8 ! .3$ \\
\hline & $2.270 .33 !$ & 1071.11 & $2.1+10.972$ & 100.0 & \\
\hline
\end{tabular}

Commerce et transports. - Nombre des erploitutions en 1852 et 1598.

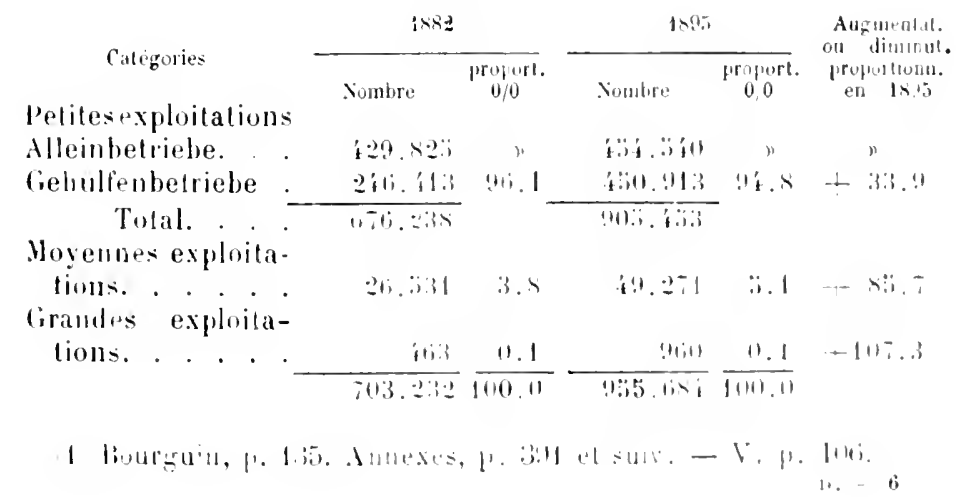


Il faut entin signaler l'influence lrès évidente qu'a exercée sur la concentration dans la banque la formation, en ces dernières amnées, des nombreux cartells allemands, influence que le rapport général de la Dresdner Bank de 1902 nous fait pour ainsi dire toucher du doigt: "Notre attention, dit ce rapport, était depuis longtemps dirigée vers linxtension de notre sphère d’intérets dans le grand district industriel du lihin el te $\mathbf{W e s}$ phalie dont ľimporliance pour l'ensemble de la vie ćconomique allemande va encore grandir par les groupements existants ol les groupements en formation..." In 1897, on comptait en Allemanne 230 à 250 cartels, 300 en 1902. L’influence en particulior du Rheinisch Westlälisches Syndicat sur la concontration de la Banque, n'est poinl niable: c'est sa formation qui décida la Deutsche Bank à s'assurer une lerme participation dans la Bergisch Märkische Bank et Ie Solılesischer Bankverein (1). La comslibution du West fälischer Kokssyndicat, du Rheinisch Irestfalisches Roheisensyndicat n'a pas peu contribué également à l'extension des grands instiluts bertinois dans colle région industrielle (2).

Lir conclusion se tire d'olle-mime: le développement de ces différentes industries. le mouvement de concentration qui s’y révòle ont nécessité des capitaux et un erédit sans cosge croissants anx aussi ; les banquos directement intéresséces à ces diflírentes entreprises ot comptant leurs principaux ahols au nombre de leurs conseillers ont dô suive le même exemple et progresser d'me façon parallelo, - el, soit far desaugmentations de eapital propre,

(1) V. P. 128 .

2) V.P. 132 . 
soit par des atliances of des contrats, tmover lo mond de faire fatce à ces nouveaux he-obus. Lat roncentration danlat banque a, par le fait mime de are rapportio directs an étroits qui lient en Allemagne. lindu-trie at le commeree a la hanque, reçu ume impulsion numbelle et la vitessed de son cours s'est trouvée singulièremunt arerue.

\section{II. - Causes particulieres.}

\section{A. - Laeliom législatiore (Losis de funaners. Irepotyesets. Birsengesetz).}

Au cours de ces dix derniipes années laction législattive est renue singuliorement penforeer la suprériorite technique des srandes banyures. loverses lois de linalues. la loi sur le dépot et surtout la loi sur les Bourses, ont, sinon dans l'esprit de leurs auteurs, du moins incontestatblement dans leurs effete, an comme conséquence diaccentuer linfériorité des petites banques vis-at-vis des grandes et de précipiter le mousement de concentration.

\section{Lois de finaners.}

Les affaires de Bourse of les ámissions ont iti sommine

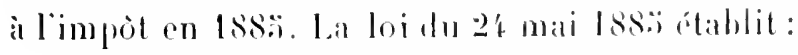

10 Un impòt de timbere sur les tilres (lieishesstempels-

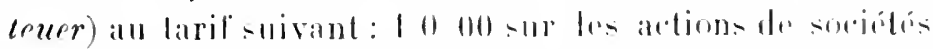

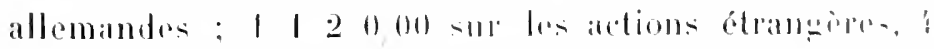

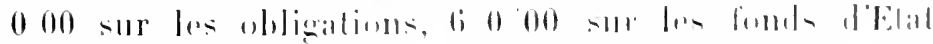

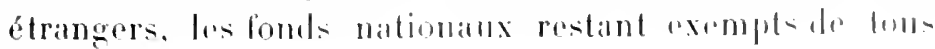
droits.

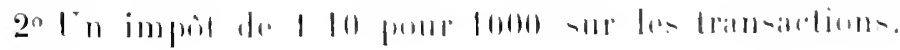


(Umsatzsteuer) qui se prélève lors de la conclusion de tout marché de bourse au moment où l'intermédiaire remet à son client une note relatant l'opération conclue (Schlussnote).

La Reichsstempelgeselz du 27 avril 1894 doubla cette dernière imposition (2/10 0/00 au lieu de 1/10 0/00), qu'elle fit de plus porter désormais sur chaque fraction de 1000 marks, n'exemptant de l’impôt que les transactions au-dessous de 600 marks.

La loi du 14 juin 1900 vint enfin porter à $3 / 100 / 00$ le Schlussnotenstempel pour les transactions sur les actions et obligations de sociétés étrangères ainsi que sur les actions de mines.

Déjà en 1884, à l'innonce de l' Umsatzsteuergesetz la Gazelte de Fruncfort prévoyait comme conséquence directe du nouvel impoot, la concentration du trafic de banque entre les mains de quelques banques puissantes $(\mathbf{1})$; à plus forte raison ces relèvements onéreux de l'impôt sur les transactions ont-ils encore accentué cette tendance et aggravé l'infériorité des banquiers de province vis-àvis des grandes banques; cela, au double point de vue suivant:

En principe, le banquier local "doit directement ou indirectement, faire supporter a ses clients trois fois les droits ordinaires: une première fois, pour l'opération passée par le banquier d'une place de bourse avec une autre persomne de cotte place: une seconde, pour celle passée par le banquier de la place de bourse avec ledit banquier local: enfin, une troisième fois, pour celle que

(1) Frunkfurter Zeitung, 26 mai 1884. 
passe lo hanquier local avereson client. Si nous supposons

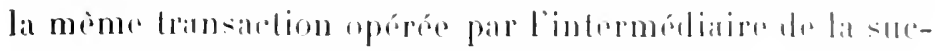

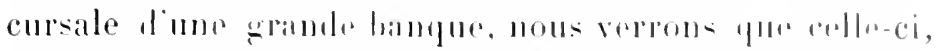
representécur bes places de bourse par le sioge contral on d'autres succursales n'aura a fare supporter au cliont

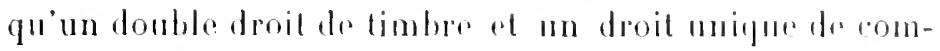
mission (1).

Aussi bien, dans beaucoup de cas, une grande banque au trafic considerable pourra compenser les ordese; ollo fera un seul bordromar a nom de deux chents, au lieu de faire deux bordereaux en son nom arecehacun de deux clients, économisant ainsi frais de comrtage et droits de timbre (2). Chaque grande banque, dit-on, devient ainsi une Bourse: " hit. qui peut dautre part presenter certains inconvénients. nin a pas moins assure and grandes banques ume suprépiorití incontestable dans la lutle de la concurrence: la clientilo a délaissó très vile les petits hanquiers, elle sest adresséc dipectement aux grandsétablissements ou à leurs suecursales qui lui laisaient de moillemres comdilions ol exigeaient ale moindres provisions.

l'antre part, lo somei l'élargir le champ de la compensation a poussé les grandes banques ir crér des succur-

1) "La loi a reconum les relations correalesflin met" qui existaient entre maisons de differentes places, cerci a permis lattemaer en partie les effets de la concurrence cribsante; el cepemlian si deux wrandes ban-

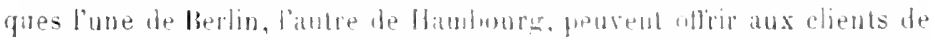

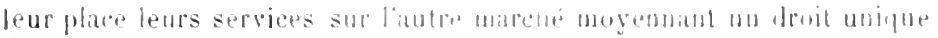

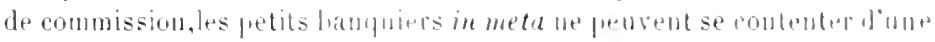
provision aussi minime. "Sitgus, Etme iconomigne el jublique sur

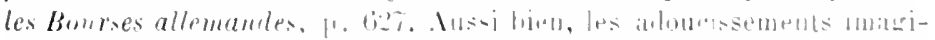

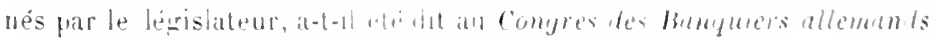

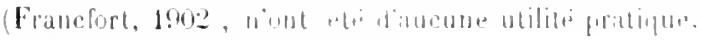

2 V.Meber, op. rit...11. lit. 
sales et des agences, à multiplier les alliances avec les banques de province qui acceptèrent d'autant plus volontiers qu'elles éprouvaient, elles, le besoin d'avoir des correspondants a Berlin et dans les autres places de Bourses. "Les raisons qui nous ont poussi à conclure un accord avec la Deulsche Bank, dit le rapport général de la Bergisch Märkische Bank (1897), se résument aiusi : cette combinaison laisse intactes notre indépendance et notre organisation; elle ne nous fait point nous détourner de notre vrai terrain d'action par la préoccupation de fonder une filiale dans la capitale."

Le coup porté anx petıls banquier's fut d'autant plus rude que par la nature de leurs allaires, ils étaient plus porlés aux affaires de Bourse: une tranche importante de leur commerce, les abbitrages, fut particulièrement atteinte (1); saus parler des nombreuses formalités qui accompagnèrent les lois de 1883 et de 1900 (droit d'inspection accordé aux agents du fisc, examen des livres, etc...), et dont ils souffrirent plus que les banques par actions. Nous allons retrouver ces mèmes effets de la législation fiscale aggravés encore par le Depotgesetz et le Börsengesetz: nous pourrous ensuite préciser ces résultats de l'action législative, au point de vue qui nous intéresse particulièrement, en montrant le recul des petites banques et les progrès des grands établissements.

(1) Devant la destruction des arbitrages, on a bien essayé des palliatifs en accordant des restitutions de taxes: mais nous voyons comment celles-ci mêmes accusent une diminution constante : elles se montaierıt à 111.316 marks en 1895 : elles ue représentent plus en 1903, que 55.000 marks. 


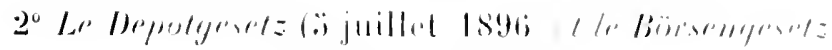

\section{(2) juin Is!);}

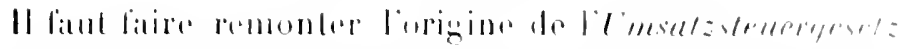

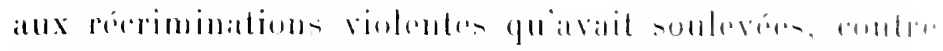

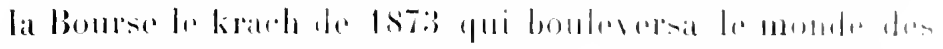

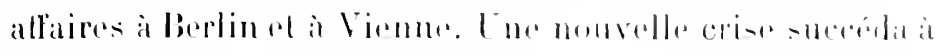

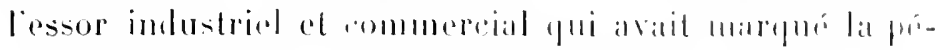

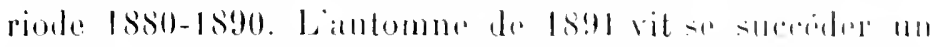

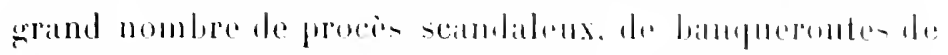

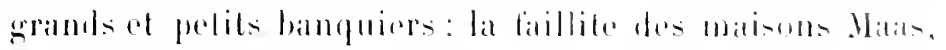
Hirschledel at Mollt, la condammation du directend de la

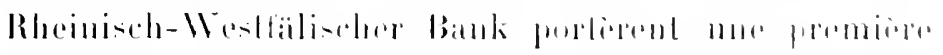

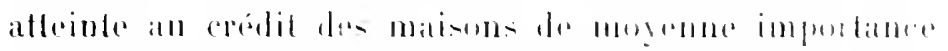

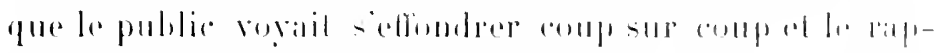

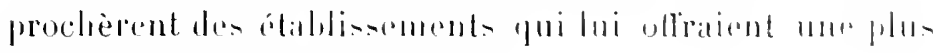
grande siecurilí apparinde.

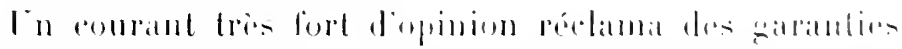

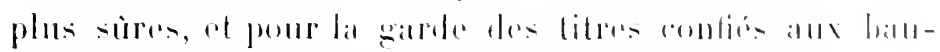

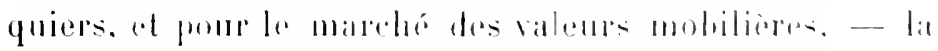

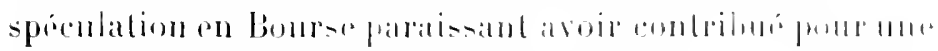

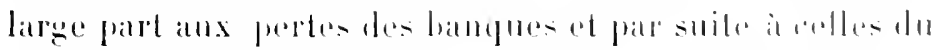

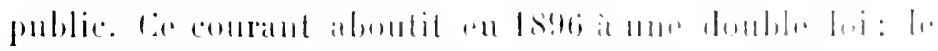

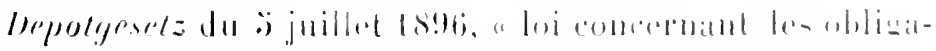

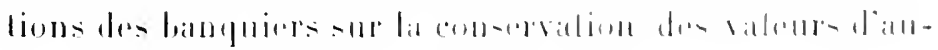

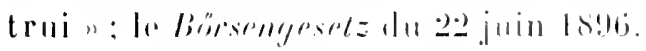

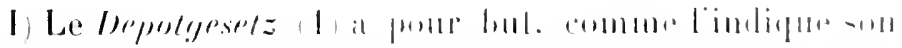

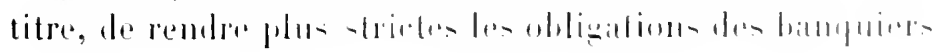

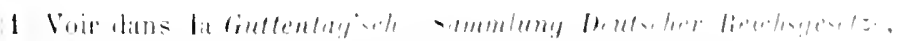

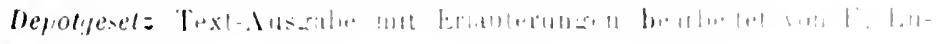
seliskin. 
au point de vue de la garde des titres, afin d'empêcher la confusion des titres achetís pour le client avec ceux qui servent aux banquiers pour leur trafie journalier et de donner aux transactions la forme juridique qui correspond à la volonté réelle tes parties. Dans ce but, la loi a établi une série de mesures spéciales: obligation pour le banquier de fournir au client, dans les trois jours, la spécilication numérique el détaillée des valeurs achetées, tenue de livres spéciaux où restent consignées ces indications, classement et séparation des dossiers par des désignations matérielles apparentes.

Pratiquement, nous arons vu, à la maison R. Varschauer de Berlin, distinguer trois sortes de lépôts:

Dépót A : sans individualisation complète des titres, couverture des opérations faites par la banque avec le client.

Inépoit B : qui comprend les elfets achetés pour le client, dont il possède les muméros el qui ne peurent servir de base de crédit dans les relations courantes.

Stücke konto qui comprend les effets disponibles

Disons dès maintenant que cette réglementation étroite a, d'une part, beaucoup gèné les maisons de petite et moyenne importance, et de l'autre poussé encore aux augmentations de capitaux daus les grandes banques: elle nécessite en effel un fonds de ronlenent plus considérable, des obligations de trésorerie plus lourdes; la spéculation exige sonvent la liaison lors du contral, de capitaux que le client n'a point toujours entièrement disponibles: la disposition de sommes plus importantes est ainsi devenue, sinon nécessaire, au moins utile et trìs fructueuse (1). Enfin, détail qui a sa valeur, les grands établissements 
bien outillés ont pu largement divelopper leurs installittions de coffres-forts gu'ils toment anx clinds disiremx d'me sécuriti plus complite entore on qui ne vimlent point payer de droits de garde. Celte combinaison a attiri aux grandes banques une notable clientible il estasos naturel en eflet, quand on a ses valeurs at ses capitaux lans une banque, de sadresser ì elle pour le paiement des coupons, les envois de fonds ot les plarements successifs.

II. L'effet centralisateur du Börsengesetz est beaucuup plus considérable encore. Nous avons dit l'émotion produite partes banqueroutes et les scandales de l'anníe ls9l; le parti agrarien prit la lète da monvement de rélorme et saisit le Reichstag de multiples propositions alynt pour but de réglementer les contrats diffrírentiels et de combattre les marchés à terme qui cachaientdesopérationsde jeu: on voulut exchure de la Bourse les joueurs el en bannir les valeurs exotiques. Une commission nommée en férrier 1892, déposa le 3 décembre 1898 une proposition de loi qui devint le 22 juin 1896 le Börsengescetz (1).

Il n'entre pas dans le calle de notre sujel d'étudier dans le détail la loi sur les Bourses. Nous neen lixerons que les traits généraux nécessaires pour comprendre l’inlluence qu'elle a exercée sur le monvement de concentration.

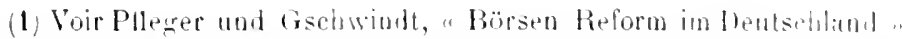

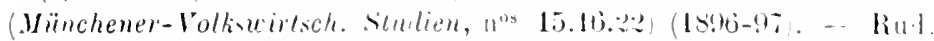

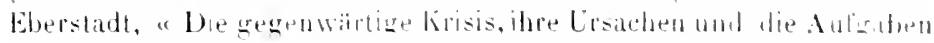

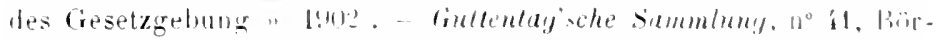

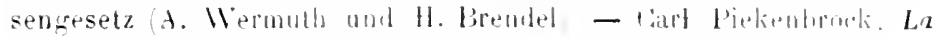
loi allemande sur les bourses el nes effrts. These. Lausanne, tonter. - All. Weber et A. E. Silyur, op. cir. 
Leux des innovations établies par la loi de 1896 nous intéressent parliculièrement: la première concerne les lormalités d'admission à la Bourse. Pour éliminer les joucurs, et d'autre part, pour donner une base solide aux marchés à terme en excluant l'exception de jou dont la jurispundence avant la toi faisait un emploi abusif dans tous les marchés différentiels, la loi a introduit l'institution du registre de bourse. Article : $\%$ : " Il sera ouvert près de chaque tribunal compétent pour la tenue du registre de commerce, un registre de bourse pour les marchandises et pour les valcurs." - Artiele $6: 30$ : "On inscrira sur le registre de bourse les noms, prénoms, professions et domiciles des personnes qui désirent faire des affaires de bourse ì lerme surles marchandises ou sur les titres (ou s'il s'agit d’ume socióté commerciale, sa raison sociale)." Liarticle 66 pose la sanction : N Nest pas reconnue comme constituant une dette valible, toute opération de bourse à terme concernant une branche d'affaires, pour layuelle les deux parties n’étaient pas inscriles sur un registre de bourse, au moment de la conclusion du marché. "

La seconde innovation est d'une gravité plus grande encore. Article 48: "Le marché à terme de bourse des parts d'entreprises minières ou industrielles est interdit." Article :0 : "Le marché à terme de bourse sur les parts d'autres sociélés ne peut être permis que si le capital de ces sociétés s'élève au moins à 20 millions de marks."

Quels furent les eflets de ces deux principales dispositions de la loi de 1896 ?

a) En ce qui concerne l'obligation de l'inscription au registre de bourse pour pouvoir opérer à lerme, "son principal résultat ful de faire disparaitre de la bourse tous ceux 
qui, de peur de diminuer leur consintéralion dans le mondr.

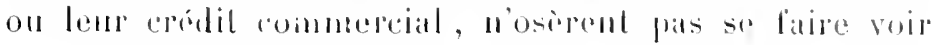

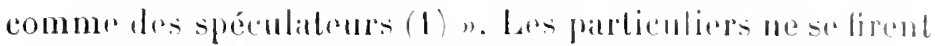
point inserire 2 el les petiles batnques al las pelits bian-

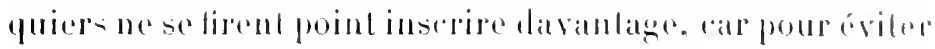
lexeeption de jen. il fant que les deux parties soinat inscrites:

"Les disposilions sur loregistre de hourse, déclatrient a l'unanimilé les banquiers allemands rómis on congres

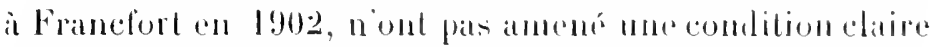
el sime de la jurisperudence; - tout an contraire. Ises banques de province natratent ancune raison de se falire inserire all registre de bourses, paree que la portion la plus

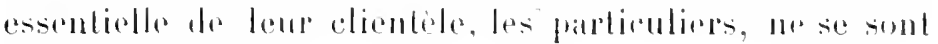
point faitinserise."

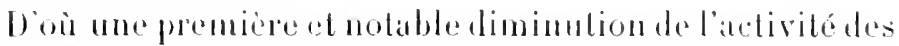
pelits banquiers, d'aulant plus sensible pour eux que pare la nature mèmu de lours opérations ils sont phus portés auxallares de homse. La non-inseription na daillenrs

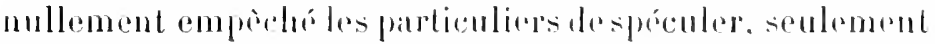
ils le firent desormatis an moven de grambes brompues, qui

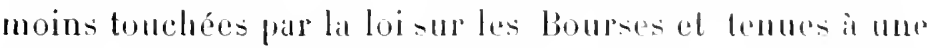

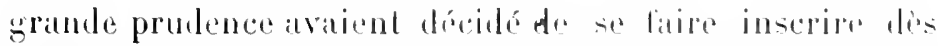
1597. Les formalités pour l'anlmission il la liomse rendues

1) Piekenbrock, op. cil., p. 131.

$\because$ Le nombre des particuliers inserits an regintro he bourse des raleurs était, pour l'ensemble des linnrses allematuder. de:

29 en 1898 ( er $^{2}$ janvier

21 en 1899

20 en 1900

36 eo 1901

30 en 190:

30 eा1 1:10:3 
trop sévères et trop compliquées n’ont donc eu comme résultat que d'accélérer le mouvement de concentration sur les grands marchés et notamment à Berlin.

६) Mais ce fut surtout l'élimination des aflaires à terme qui vint accroitre lactivité les grands établissements et accentuer l’infériorité des petits banquiers.

L'article 48 a certainement causé une diminution considérable des affaires à terme, comme on peut s'en rendre compte par les tableaux suivants (d'après Piekenbrock) :

Mouvement d'affaires de banques 't banquiers allemands pour des opérations a lerme faites dans les Bourses allemandes.

I

De 12 bunques de Berlin, Francfort ou llambourg.

$\begin{array}{ccc}\text { Années } & \text { Tutaux } & \begin{array}{c}\text { Chiffres } \\ \text { relatifs }\end{array} \\ \text { 1893. } & 5.849 .741000 & 100,0 \\ \text { 1894. } & 6.572 .109 .000 & 112,3 \\ \text { 1895. } & 9.738 .992 .000 & 166,5 \\ \text { 1896. } & 6.011 .082 .000 & 102,8 \\ \text { 1897. } & 5.489 .205 .000 & 93,8 \\ 1898 . & 5.746 .882 .000 & 98,2 \\ 1899 . & 6.881 .091 .000 & 117,6 \\ 1900 . & 3.706 .336 .000 & 63,4 \\ 1901 . & 2.066 .286 .000 & 35,3 \\ 1902 . & 2.449 .733 .000 & 41,9\end{array}$

\section{II}

De 21 banquiers de Berlin, Erancfurt ou Hambourg.

$\begin{array}{ccc}\text { Années } & \text { Totaux } & \begin{array}{c}\text { Chiffres } \\ \text { relatifs }\end{array} \\ 1893 . & 3.13+.196 .000 & 100,0 \\ 1902 . & 1.104 .836 .000 & 330,3 \\ & \text { III } & \end{array}$

De 11 banques ou banquiers de province.

1893. 441.283.000 100,0 1902. $131.111 .000 \quad 29,7$

On remarquera en premier lieu, que la diminution des affaires di lerme s'est surtout fait sentir pour Jes banquiers privés et les banques de province.

Mais cela ne veut nullement dire que les spéculateurs, les joueurs, ne spéculent plus: on constate au contraire une augmentation corrélative du marché au comptant surtout sur les valeurs pour lesquelles le marché à terme 


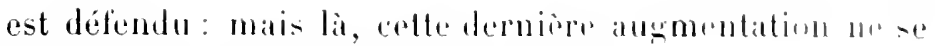
vérifie que pour les gerandes bangume:

Opérations au complant de s' banques de Brelin. Franefort oul Hambsurg.

\begin{tabular}{|c|c|c|c|c|}
\hline Années & Totaux (marks & $\begin{array}{l}\text { Chitlires } \\
\text { relutils }\end{array}$ & 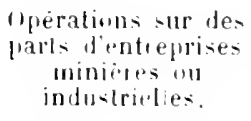 & $\begin{array}{l}\text { Chilfres } \\
\text { relatils }\end{array}$ \\
\hline 1893. & 2.170 .958 .000 & 100,11 & 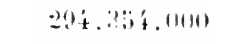 & 1110,0 \\
\hline $189 x$ & 2.734 .269 .000 & $1 \geq 6.9$ & +73.2.84.0001 & $161,: 3$ \\
\hline $189 \ddot{.}$ & 3.790 .638 .000 & 17,6 & s!ti. 209 (110!1) & 3013,8 \\
\hline 1896. & 3.335 .863 .000 & 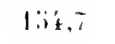 & 9162.1 .0000 & 311,3 \\
\hline 1897 & $\{.061 .02: 3.000$ & $18 \pi, 1$ & $1.2+0.1 \div 2.000$ & 221.3 \\
\hline 1898. & 4.668 .222 .01010 & $21: i, 11$ & 1.098 .218 .01000 & $3+1.8$ \\
\hline 1899 . & 3.862 .756 .000 & 2711.1 & $=620.9+3.0100$ & $\$ 90, \dot{t}$ \\
\hline 1900 & '́.98't.693.000 & 229,6 & 2.51\%.2'0.000 & 8.11 .1 \\
\hline 1901. & 3.942 .663 .000 & 181.6 & 936.826 .000 & 318.3 \\
\hline 1902. & 4.413 .262 .0000 & 203,1 & $91+9+1000$ & $310, x$ \\
\hline
\end{tabular}

De 22 banquiers de Brelin, Franctort ou Hambourg:

$\begin{array}{rrrrr}1893 . & 396.8113000 & 100,11 & 96.201 .11000 & 100,0 \\ 1902 . & 547.752 .000 & 138,0 & 258.620 .1000 & 268,9\end{array}$

De 92 banques ou banquiers de province:

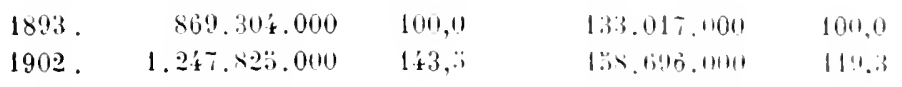

Ainsi, diminution pour les petits hanquiors du nomber

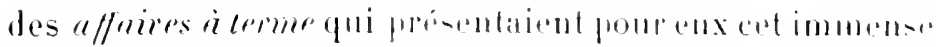

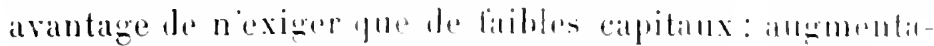

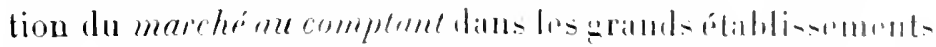

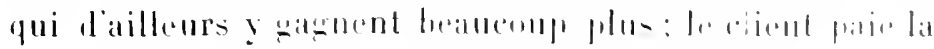

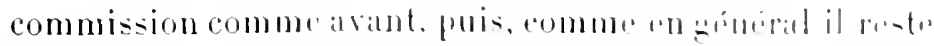

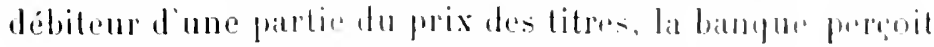

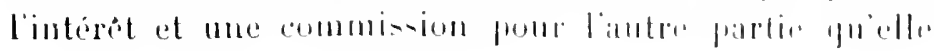


avance; enfin clle a les titres en dépòt et se fait payer un droit de giade.

Rappelons encore cet avantage des grandes banques que déjà nous avons signalé; elles exécutent par compensation la majorité des ordres et il n'y a que les soldes qui viennent en bourse.

"Le résultat le plus certain de l'interdiction du marché à terme de valeurs industrielles et minières est l'accentuation du triomphe des grandes banques sur les petites et sur les moyennes maisons : la liaison des capitaux devient souvent nécessaire, les banques de courtiers ne sont point disposées à faire grand crédit à des maisons n'offrant pas la garantie d'énormes capitaux, car la notion de confiance personnelle a sur le marché allemand une bien moindre place que sur le marché français. Peut seul etre mandataire de nombreux spéculateurs le détenteur de grands capitaux (1)."

La nécessití de se procurer ces énormes capitaux qu'exigeaient un accroissement de leur activité générale, l'augmentation du marché au comptant en particulier (2),

(1) Sayous, op. cit., p. 464.

(2) D’autres dispositions plus particulières du Börsengesetz sont également venues nécessiter une augrmentalion des ressources propres des banques; l'article 39 par exemple, qui décide que l'admission à la cote de la bourse d'actions d'une entreprise transformée en société par actions ne pourra être permise avant qu'une année ne se soit écoulée depuis l'inscription de la société sur le registre de commerce ni avant la publication du premier bilan anuel et du compte des profits et pertes. La loi a été aisément toumée ; on attend le terme exigé pour faire l'émission officielle el entre temprs on rend les titres en sons-main. Mais seules les grandes banques ont un capilal assez considérable pour en immobiliser une partie daus ces opérations, et une elientèle assez étendue pour pouvoir placer tous ess titres. Nouvelle inférinrité des petites banquars vis-it-vis des grandes. - V. Piekenbrock, op cit., p. 93 et $1: 6$. 


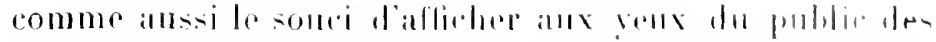

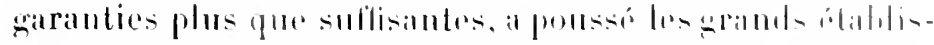

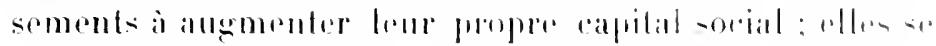

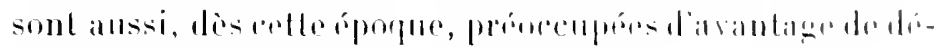

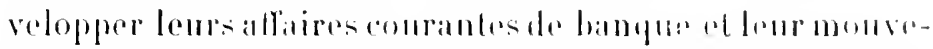

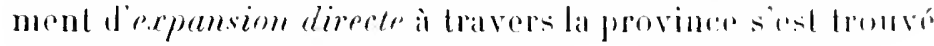
par là singulièrement accentur.

Voici le bableau des angmentations dreapital des banques allemandes cotérs a la Bourse dr brerlin avere un cat-

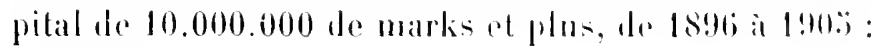

\begin{tabular}{|c|c|}
\hline & $\begin{array}{c}\text { Cappital-actions } \\
\text { entiopement } \\
\text { velsi }\end{array}$ \\
\hline Binques & fin $18,36 j$ lin $19010 \mathrm{i}$ \\
\hline
\end{tabular}

Reichsbank. . . . 12010.1100.0101) 180.0100.000

I) eutsche bank . . . 101.01010.0101)

Discontogesellsphaft. . 11:; 1600.0161$)$

180.0000 .0001

Uresther lank. .

Darmslidter Bank.

Scha a fl ha a sen s'cher

bankverein. . . .

Berliner Handelstesells. chaft.

Commerz u. Disconto bank. . . . . .

Sational bankfürl)eutschland..... . Bergisch - Mirkische Bank. . . . . . l'fiilzisilie liank. . . Barmer bankverin. Mitheldentsolue tiredil hank. . . . . . Rheinische Disontogesellswhaft. 180.0000 .11001 8.i.19014.1116) 100.00000010

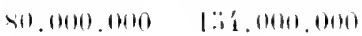
Allgmentation depuistentrie en viguente die la lol sll les bourses.

(i) 111111.11001 (i) . $14+(1), 0011$

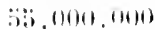
7:i. 0)(111.1110 $\because \mathrm{i} .00010011$

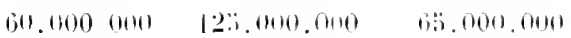
$80.1100 .000 \quad 100.0001 .0001 \quad 20.0001 .1100$ $311.000 .000 \quad 8 \% .1000 .01101 \quad 3.1 .1011 .000$

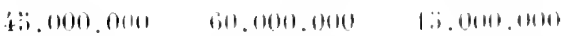

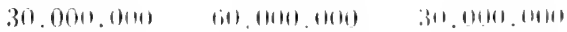

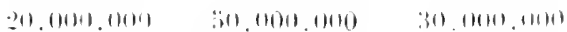

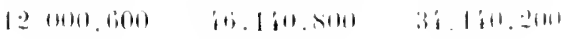

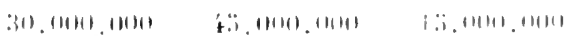

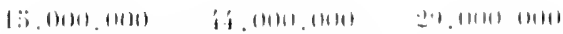

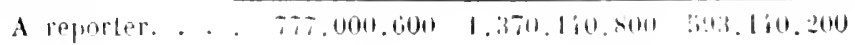


Report. 770.000 .6001 .370 .140 .800

593.140 .200

Essener Credil anstalt.

$15.000 .000 \quad 40.000 .800$

25.000 .800

Deulsche Effekten und

Wechselbank ... .

$15.000 .000 \quad 30.000 .000 \quad \mathbf{1 5 . 0 0 0 . 0 0 0}$

Schlesischer Bankverein .......

Breslauer Discontobank

Hannoversche bank. .

Wurltembergische Vereinsbank. . . .

Uresdner Rankverein.

Dentsche Nationalbank.

Breslauer Wechslerbank

Duisburg - Ruhrorter

bank. . . . . .

Ostdeutsche Bank. . .

Westfälische bank. . .

27.000 .000

30.000 .000

3.000 .000

22500.000

25.000 .000

2.500 .000

$12.000 .000 \quad 22.500000$

10.500 .000

$\begin{array}{rrc}18.000 .000 & 18.000 .000 & - \\ 8.000 .000 & 18.000 .000 & 10.000 .000 \\ 11.250 .000 & 15.000 .000 & 3.730 .000 \\ 12.000 .000 & 12.000 .000 & - \\ & & . \\ 3.000 .000 & 12.000 .000 & 9.000 .000 \\ 7.500 .000 & 10.000 .000 & 2.500 .000 \\ 6.000 .000 & 10.000 .000 & 4.000 .000\end{array}$

Norddeutsche Credit

Anstalt. . . . .

Rheinische Bank. . .

Total.

\begin{tabular}{crr}
- & 10.000 .000 & 10.000 .000 \\
- & 10.000 .000 & 10.000 .000 \\
\hline 979.250 .600 & 1.721 .641 .600 & 742.391 .000
\end{tabular}

Soit en ces huit annérs, une augmentalion totale de 742.391 .000 marks (4\%4 millions de marks pour les seuls Instituts berlinois).

Le montant des provisions payées par la clientèle pour l'exécution des ordres de Bourse a suivi dans les cinq grandes banques berlinoises la progression suivante: (Marks)

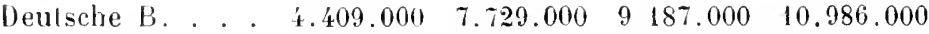

Discontogesellsch. $2.741 .000 \quad 4.262 .000 \quad 4.580 .000 \quad 5.277 .000$

Dresdner B. . . . 2.18:.000 $5.503 .000 \quad 5.104 .000 \quad 6.538 .000$

Schaalfhaus. B. . . 1.332.000 2.229.000 2.449.000 3.430 .000

Darmstidter B. . . $1.030 .000 \quad 1.506 .000 \quad 2.362 .000 \quad 3.427 .000$

De deux milliards et demi de marks en 1896, les opérations d'arbitrage sont tombées à 5450 millions de marks en 1900. 
Les dix grantes banques berlinoises qui complationt in 189:3, 21 succursiles, 32 carisses de dépouts et lï participat-

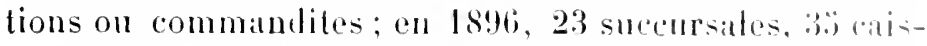
ses de dépòts et 16 participations on commanditos dim

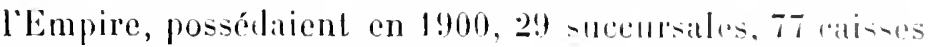
de lépòts et 24 parlicipallions on commandites: en 1902,

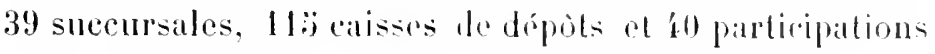
ou commandites.

D'autre part, à la lin de la période 1S911-1896 on comptait 6:3 banques et filiales de plus al :32 banques privén et liliales de moins qua commencement de celle prériode (1890).

A la fin de la périorle 1897-1902, 207 banques et liliales s'élaient fondées, 123 banquiers privés et liliales araient disparu.

Le nombre des girokontos à la Reichsbank, qui sétatit aceru dans la période 1877-1896 de:300 girokontos (banquiers privés), 11 g̈ girokontos banques et liliales', augmentait dans la période $1896-1902$, de 29 siroliontos seur lement (banquiers privés el liliales) contre 2 l' girokoutos (banques el filiales).

Le rapport de MM. Riesser, Hassek al M̈ittner all Ceretral Verband (1903) a enlin mis en lumirire diutere faits intéressants: le nombre des télégrammes axpédiés dans les bureaux de bourse accuse une diminution constanle

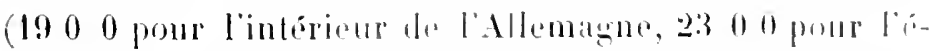

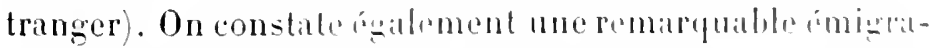
tion des alfaires de spéculation el de platentent. En ls!l:3,

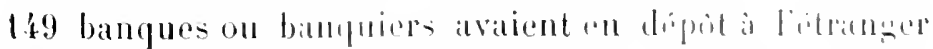

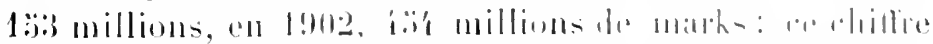
des dépots a létranger qui alteignait labi miblions pour 
18 grandes banques, atteignait en 1902, 608 millions de marks.

On voit parces différents aspects du progrès des grandes banques et du recul de l'activité et du nombre global des banquiers privés depuis 1896 , la notable accentuation du mouvement de concentration. Sans doute ce n'est point le börsengesetz seul, même secondé par les lois de finances, qui a produit ces résultats.

Hais il a précipité la concentration : "La concentration des banques, la suppression des petites et moyennes institutions et maisons de banque, la prépondérance croissante de Berlin et des établissements qui y ont leur siège ont été des conséquences indirectes da Börsengesetz: la loi a précipité les événemenls et hàté la disparition ou l'alsorption des banquiers modestes (1). " La loi a fait passer les affaires de bourse dans les bureaux des banques, clle a poussé à diminuer le nombre des intermédiaires, remplacés par les grands établissements.

Certes ce n'était point à de tels résultats que s'attendaient les auteurs de la loi; dans leur esprit le Börsengesetz avait mission de protéger les petits et moyens banquiers contre la concurrence des grands établissements; les résultats produits furent tout différents:

"Le Börsengesetz, déclarait le Congrès des banquiers réunis à Francfort en 1902, n’a nullement rempli les buts économiques auxquels il devait servir; tout au contraire, il a eu les conséquences les plus fìcheuses. Notamment cette loi a facilité l'absorption des petits et des moyens banquiers par les grandes institutions de crédit, en mème temps yu'elle affaiblissait la force économique de l'indus-

(1) Raffalovich, Marche financier, 1898-1899, p. 248. 
trie de la banque tout entiope of quiollo lui nuisait ann-

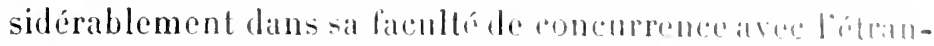
ger. "

"Lextension de la gramde expluitation ast, il e-t rati. un signe des temps, écrit le Deulsche orkomomine du 21 mai 1904, mais elle a riti accélérée par les moyens pris contre elle, moyens qui étaient desstimulants pour los grands et qu’ils supportent bien plus farcilrment qur les petits et les faibles. Les grandes bangunes sorquni-iomut immédiatement en consíquence et l'exprírience a montré

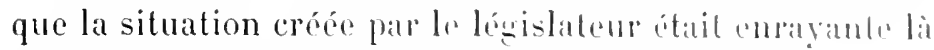
où elle aurait dù ètre stimulante et réciproquemunt."

Il convient d'ajouter qu les grands ritablisemente par raissent avoir eu des vues plus exactes sur les ranlutits futurs du Bürsengesetz: "Lal loi sur les liourses, di-ait le rapport général de la lleutsclue bank en 189:\%, n’anra pas les effets qu'en attendent ses auteurs; elle ne diminurar pas le gout de la spéculation si elle en diminue les oljets et la forme. Les grandes maisons aux capitaux importants pourront répondre aux exigences nouvelles. les moint fortes disparâ̂tront pru à peu."

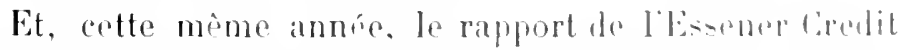

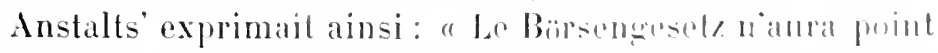

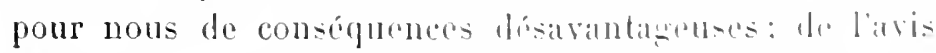
général, il nous sera plutiot farvorable carr l'entremise des opérations sur valeurs se concentrera phlus encose flu atujourd'hui auprès des grands ćtablisoments."

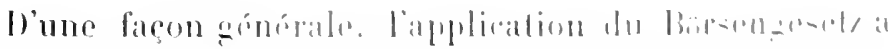

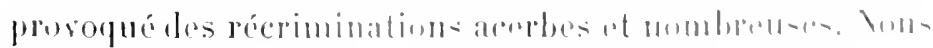

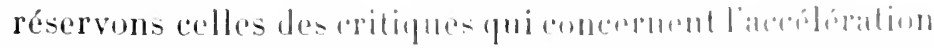
du monvement de concentration, la di-pratrition des putit 
et moyens banquiers. Ajoutons seulement que le législateur semble s'être rendu compte que sa tentative de protéger les moyennes institutions, de mieux régler les affaires de bourse et d'y rendre impossibles les opérations frauduleuses a subi un échec presque complet.Une commission de réforme nommée en février 190' a déposé dans les premiers jours de mai $190 \unlhd ٌ$ un nouveau projet de loi. Quoi qu'il en advienne, il nous semble difficile aujourd'hui de faire revenir le public sur certaines habitudes, - aussi bien, ces résultats que nous venons de constater restent toujours acquis.

\section{B. - Phénomènes internes à chaque établissement.}

Nous avons examiné sous ses deux aspects technique et économique, la cause générale du mouvement de concentration; aussi bien n'est-ce lì, avons-nous dit, que l'expression particulière que prit pour le commeree de banque en Allemagne la tendance générale à la concentration que l'on peut constater dans tout le domaine économique. Les causes particulières que nous avons indiquées n’ont fait que soutenir et précipiter cette tendance.

A plus forte raison, ne pouvons-nous considérer que comme des causes très secondaires, ou plutôt même comme de simples occasions, toute une série de phénomènes internes ì chaque établissement : la maladie, le décès d'un des chefs d'une maison particulière, le départ d'un associé désireux de réaliser sa part, voire l'ambition chez un banquier local de voir rejaillir sur luile prestige d'un grand établissement, etc... On peut sans doute, lors de l'examen de telle fusion isolée, de telle absorption de banque par une autre, de telle commandite déterminée, être ame- 


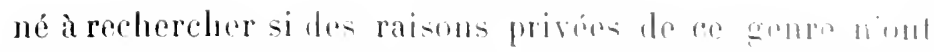

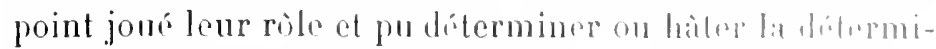

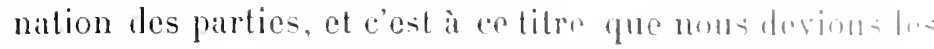
indiguer ici. Mais il est impossible de romloir aplipune. uniquement par les causes de cotto portion, un lait all-i

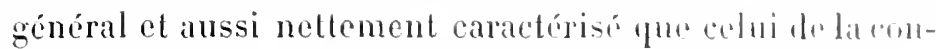
centration dans les banques allemandes.

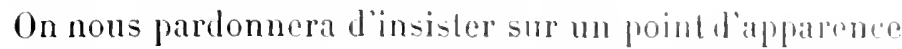
aussi simple : e'était cependant, croyons nous, mbersinire, ear d'autres sont. ou ćlaient, d’un atis dillórent. Hams des premiers et des rares articles francais iroits sur la

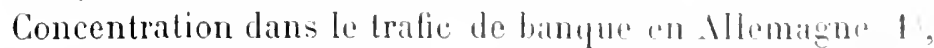
M. A. E. sayous prétendait expliquer cortains fails importants de concentration par ces causes tout a fait particulières. Ce ne sont pour lui "qüune situation de l'amille et un état desprit qui déciderent de la disparition de la maison Dreyfus " de Franclort, absorbé on t s.gs par la lommerz und Disconto Bank. - "Des motifs personnels, mais plus obseurs expliquent ógalement l’absorption do lit banque Landau par la National Bank fiïr loulscluland. "Il. Sayous explinue la commandite de la maison Ri. Varschauer par la Darmstidter Bank de lis facon suivinte:

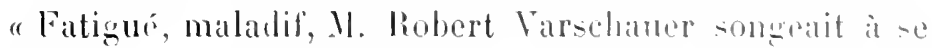
retirer... la commandite de la banque do [ram-tallt lui prermit de digager ses capitanx sims peinu ut il hom "omple w. - Et enfin: "Si leschel's do la vieillo ot solide mai-on

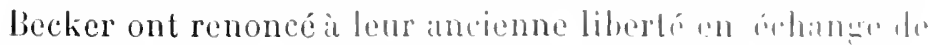

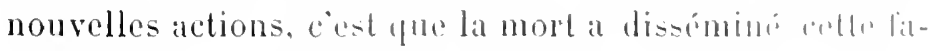

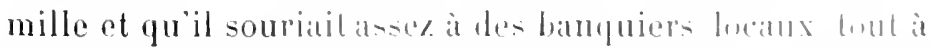

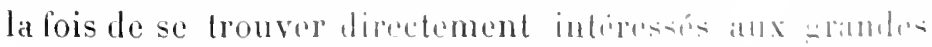

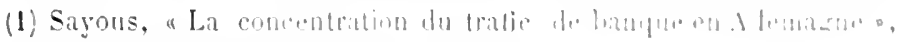
Journal des Eronomives, 15 jastier Ji!n. 
opérations financières de la Discontogesellschaft et de voir rejaillir sur eux le prestige d'un nom universellement connu ot honoré."

Nous ne nions point, tout au contraire, la valeur qu'ont pu aroir dans les événements rapportés ces raisons purement privées; mème déterminé par les causes les plus générales, l'homme ne passe à l'action que sous l'influence de motifs ou de mobiles personnels. S'ensuit-il que la cause première et générale n'existe pas ? Doit-on, à cause de sa généralité même et parce qu'elle explique tout un ensemble de faits, la qualifier "d'explication rudimentaire " et en faire bon marché ? Il est imposible, dit II. Sayous, d'expliquer ainsi (c'est-à-dire, en invoquant une cause générale), pour quels motifs plusieurs grandes banques privées ont ou disparu ou pris un caractère nouveau. "C'est exactement le contraire qu il faudrait dire : la cause générale intervient là précisément où les motifs particuliers n'existent pour ainsi dire pas.

Aussi bien, à dire vrai, les raisons données par M. Sayous n'expliquent-elles point ce qu'il faudrait expliquer, à savoir pourquoi ce sont des sociétés par actions, et toujours les mêmes, au Sud conme au Nord du pays, qui viennent ainsi prendre la place des banques particulières. Nous pouvons dire de l'Allemagne ce qu'on a dit avant nous de notre pays: "Ce n'est pas d'aujourd'hui que de petites maisons font faillite, ou cessent leurs affaires ou que leur chef vient à décéder. Mais autrefois, d'abord les petites banques mouraient moins nombreuses et ensuite quand elles mouraient, d’atres petites banques se créaient et les vides qui se produisaient élaient ainsi comblés à mesure (1). "Or, il n'en est plus ainsi. Pourquoi ? C'est ce

(1) Naurice Saurel, op. cit., p. 124. 
qu’il faut éclaircir et non énumérer, firt-on sir du les connaitre avec certitude, les rasons privés yui ont pu causer la disparition d'une banque particuliore. Inutih. croyons-nous, d'insister longuement sur ce point.

Enfin, et ceci peut nous servir de preurea contrarin, las prédictions de II. Sayous ne se sont point rúalisécs. "L." mal, disait-il, n'est pas aussi vif guon se le figure; il peut d'ailleurs s'arreter des lemain; le recul du cours dos actions de la Deutsche Bank el de la Discontogesellschaft, durant les derniers mois, semble montrer une certaine lassitude du public; quamel les capitalistes ne romdront plus guère dactions le banque, lextension de cellus-ci se tronvera arritée! L’histoire a des retours impossiblus i prévoir !. Le mal - si mal il y a véribubloment - a persisté et la plupart de ces banques privées que .l. Sayous nous montrait comme devant résister victorieusement aux grands établissements ont aujourd'hui disparu, absorbies par ces grands établissements.

L'A. Schadthausens'cher Bankverein s'est allié à la Dresuner Bank. "La riche maison Oppenheim " a été transforméc en société en commandite par actions et l'un de ses gérants est entré au conseil de la Discontogesellschaft. La maison Erlanger a été absorbée par la Drestner bank en 1904. La Discontogesellschalt a absorbé en 1902 la banque Rothschild de Franctort, on 1903 la maison sehultze Wolde de Brème ot transformé enlin en lan' la

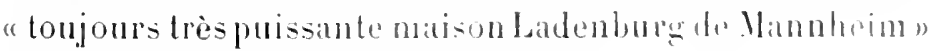
ensïddeutsche Jiscontugasellschaft.

Ces quelques exemples - que noms nowons par whoisis

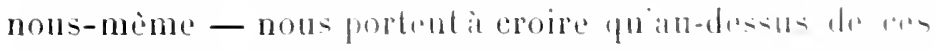

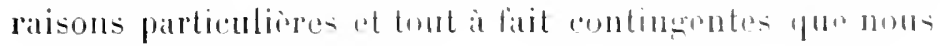


avons définjes: phénomènes intrones à chaque établissement, il est d'autres causes plus générales et plus agissantes. Nous croyons l'avoir suffisamment établi. Il nous reste maintenant à examiner certains événements particuliers qui furent également, en ces derniers temps, des causes occasionnelles du mourement de concentration.

$$
\text { C. - La crise de 1900-1901. }
$$

Ces événements particuliers ne sauraient être qualifiés à proprement parler de causes ; ils apparaissent plutôt comme le terrain où ces différentes causes que nous venons d'examiner ont exercé leur action d'une façon singulièrement précise et efficace, comme le théàtre oì s'est clairement manifestée, sous l'influence de circonstances spéciales, la supériorité des grands établissements et leur puissance d'expansion, conme aussi la difficulté qu'éprouvent les petites banques à imiter leurs grandes concurrentes dans leur activité rémunératrice. Le résultat de ces événements a ćté une notable accélération du mouvement de concentration dans le trafic de banque en Allemagne. C'est pour ces raisons que nous en avons fait une étude à part. Il s’agit de la crise économique qui a sévi avec une réelle intensité dans la période 1900-1901 (1).

W'aprìs les déclarations du premier Congrès des banquiers allemands (Central verband des Deutschen Bank

(1) Voir: Die Störungen im deutschen Wirtschaftsleben während der lahre, 1900, f. f. ; Félix Hecht, Die Katastrophe der Leipsiger Bank; loeb, Die Berliner Gro:sbantien in der Krisis; - Sehulze, Die Bank Kalastrophen in Sachsen im Iahre, 1901 ; Raffalovich, Marché financier, $1901-1902 ; 1902-1903$. 


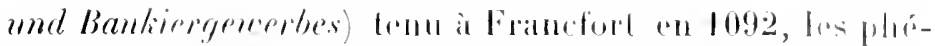

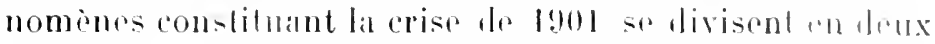
arompes: a) l’effondrement dr banques hypothroarires, d’institutions de crédit, d’untreprises industrielles, ruinón par me manvaise gestion, une administration compalste do légèrefi, en partie malhonnele, ul qui u'auratent sans doute pas échappé à leur sort en l'absence de crise grónrale. b) Le recul plus ou moins accentué de la valeur des établissements industriels, les actions el obligations iudustrielles.

"La relation entre les crises financiores et les crises commerciales et dallaires, dil Bernstein, n’a pas encore ché sulfisamment celaireie pour yue l'on ait pu dire arece certitude d"un cas concrel queleonque oì deux crises coüncidarent, que ce frit directement la crise commerciale, cest-à-dire la surproduction, qui avait causi la crise financière. Dans la plupart des cas ce ne fut visiblement pas la surproduclion proprement dite, mais la sur-spéculation, qui paralysail le marelé linancier et qui ainsi déprimait l'ensemble dos allaires (1). "

Il parait bien en awoir étí ainsi en 1900-1901. De 1896 ¿̀ $\$ 900$ il navait pas eté cré en Mllemagno moins de 13900 societés par actions; en voici le tabladu détallé ainsi que le capital quelles représentent:

\begin{tabular}{|c|c|c|c|c|c|}
\hline Années & & & & Nombre & $\begin{array}{c}\text { Capilal } \\
\text { milliers de mark }\end{array}$ \\
\hline 1896. & . . & . . . & . & $18: 2$ & $26 x .580$ \\
\hline $189 \%$ & . . & . . & . & $2: 34$ & 380.170 \\
\hline 1898. & . . & . . & . & $329 ?$ & 463.6020 \\
\hline 1899. & . & . . & . & $36 \mathrm{i}$ & $\because 4+3 ! 10$ \\
\hline 1900 & . & . . & & 261 & $3+11.960$ \\
\hline & & Tital & & 1.3991 & 1.9497 .320 \\
\hline
\end{tabular}

1) Bernstein, Sycialime theorique et sncinlademocratic pratique, p. 130 . 
C'est surtont sur les industries minières, chimiques, métallurgiques et électriques que portaient ces créations nouvelles:

\begin{tabular}{|c|c|c|c|c|c|c|c|c|c|c|}
\hline \multicolumn{3}{|c|}{$\begin{array}{c}\text { Années Mines et } \\
\text { Hauls-Fourneaux }\end{array}$} & \multicolumn{2}{|c|}{$\begin{array}{l}\text { Industries } \\
\text { chimiques }\end{array}$} & \multicolumn{2}{|c|}{$\begin{array}{l}\text { lndustries } \\
\text { métallurgiques }\end{array}$} & \multicolumn{2}{|c|}{$\begin{array}{l}\text { Industries } \\
\text { électriques }\end{array}$} & \multicolumn{2}{|c|}{ Total } \\
\hline & Nonbl. & Capilal & Nomb. & Capital & Nomb. & Capital & Nomb. & Capital & Nomb. & Capital \\
\hline 1896. & 10 & 36.038 & 16 & 20.275 & 18 & 34.662 & 4 & 13.812 & 48 & 110.287 \\
\hline 1897. & 3 & 5. 320 & 14 & 21.946 & 47 & 65.275 & 11 & 120.381 & 75 & \\
\hline $1 \times 98$. & 14 & 37.375 & 18 & 26.519 & 53 & 85.812 & 36 & วั8.182 & 121 & \\
\hline 1899. & 11 & 29.186 & 21 & 18.756 & 75 & 136.525 & 32 & 43.5 & 139 & 7.704 \\
\hline 1900. & 14 & 29.024 & 11 & 19.950 & 53 & 109.237 & 15 & 3.. 588 & 93 & 192.799 \\
\hline & 52 & & 80 & 107.446 & 246 & 431.511 & 98 & 270.200 & 476 & \\
\hline
\end{tabular}

Voici, d'autre part, le tableau des émissions d'actions de banques et d'actions industrielles émises pendant cette même période: (millions de marks).

\begin{tabular}{|c|c|c|c|c|c|c|}
\hline \multirow[t]{2}{*}{ Années } & \multirow{2}{*}{$\begin{array}{l}\text { Actions de } \\
\text { Valeur } \\
\text { nominale }\end{array}$} & \multirow{2}{*}{$\begin{array}{l}\text { Banque } \\
\text { Valeur } \\
\text { au cours }\end{array}$} & \multicolumn{2}{|c|}{ Actions industr. } & \multicolumn{2}{|c|}{ Total } \\
\hline & & & $\begin{array}{c}\text { Valeur } \\
\text { nominale }\end{array}$ & $\begin{array}{l}\text { Valeur } \\
\text { au cours }\end{array}$ & $\begin{array}{l}\text { Valeur } \\
\text { nominale }\end{array}$ & $\begin{array}{l}\text { Valeur } \\
\text { au cours }\end{array}$ \\
\hline 96. & 158 & 213 & 245 & $33 \dot{4}$ & 403 & 547 \\
\hline 97. . & 173 & 266 & 191 & 318 & 364 & 584 \\
\hline 98 . . & 273 & 372 & 310 & 521 & 583 & 893 \\
\hline 99 . . & 212 & 276 & 516 & 861 & 728 & 1.137 \\
\hline 00 . . & 138 & 155 & 298 & 461 & 436 & 6100 \\
\hline & 954 & .282 &. .560 & 2.495 & .514 & 3.777 \\
\hline
\end{tabular}

"Les cours d'émission, disait le Deutsche OEkonomist du 22 juillet 1899 , ont atteint une hauteur qu'on n'avait jumais vue. En outre c'est une rìgle constante que les titres montent bien au-dessus des cours d'émission. Nous avons déjà montré ce qu'il y a de maladif dans ces exagérations... En réalité, ce n'est pas l'idée qu'on se fait de la baute valeur du placement qui fait monter les cours, mais simplement l'idée bien arrêtée chez tous que les cours monteront encore, c’est-ì-dire la spéculation à la hausse. Le taux encore non atteint de la prime d'émission (près de $700 / 0$ ) montre que cetle spéculation a pris une extension qu'on n'avait encore jamais vue. " 
Ces chiflres nous donnent en effet une idée nettr dus

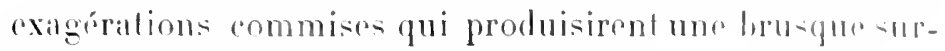
production et la crise.

Le marquis de Noailles, notre ambassadeur à Burlin. écrivait le fer mai 1901: "Le déreloppement pris depuis quelques années par l’industrie métallurgique allemande n’a pas été sans inconvénients. Tout le monde s'alcorde aujourd'hui à conslater que lon a étí trop vite. L'élívation des prix a lait naitre heaucoup d'usines nouvelles. In grand nombre danciennes entreprises ont étí developpres dans de larges proportions. Il en est rísulté mue surproduction. lin mème temps tes allitires de Chine, la guere Sud-. fricaine, renaient paralyser les transatedions de ldyemagne arec ces deux régions qui lui avaient offert depuis quelques ammées un important débouchr."

M. Galbelé, consul te france it breslau, est plus prícis encore: il écrit à la date du : juillel 1901: " La cause principale du ralentissement des alfirires est ì chercher dans l'exagération mème de la spéculation qui eut pour conséquence une hausse formilable des prix de toutes las matières ainsi que de la main-d curve, l'ayrandissement des usines existantescel la création d'établissements nouveallx.

- "Comme il était aisé de le prévoir, la production sans cesse croissante de presque toutis les industries en est arrive a dépasser singulièrement lesilemandes, lesquellas de leur còté se sont trourés singulierement ralinties parr suite de la cherté mine des produits el du minque de confiance du commerece.

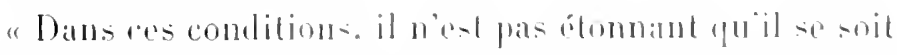

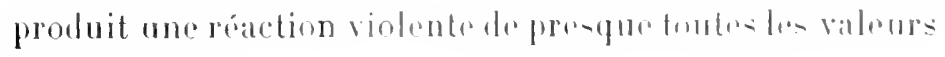
industrielles." 
Or, nous savons à quel point sont intéressées dans les entreprises industrielles les banques allemandes: un de leur's traits caractéristiques est de s'intéresser, nous l'avons vu, directement dans les entreprises de toute nature, situation qui n'est pas sans danger, car outre qu'elle immobilise les fonds fournis, si, en cas de crise par exemple, l'industriel a besoin de nouveaux fonds, la banque est obligée de les fournir pour garantir sa participation : e'est un engrenage ; une participation de 500.000 nuarks peut ainsi nécessiter l'ouverture d'un crédit de plusieurs millions. Ce sont les banques qui avaient écoulé les valeurs industrielles et facilité ainsi la création de ces innombrables affaires chimiques, métallurgiques, minières et électriques; elles avaient ouvert, souvent à découvert (ungedechit) (1) de notables crédits aux sociétés nouvellement fondées et le rapport présenté au Congrès de Francfort met au nombre des causes de la crise " ces facilités exagérées de crédit aussi bien sous la forme d'obligations assises sur des entreprises dont la capacité de rémunérer le capital engagé n'avait pas encore été mise à l'épreuve que sous

(1) Comples debiteurs en millions de marks.

\begin{tabular}{|c|c|c|c|c|c|c|c|c|}
\hline & $\begin{array}{l}\text { entsche } \mathrm{Ba} \\
\text { - à dé }\end{array}$ & & $\begin{array}{l}\text { Dresdn } \\
\text { couv. }\end{array}$ & $\begin{array}{l}\text { Bank } \\
\text { découv. }\end{array}$ & $\begin{array}{l}\text { Darnusl } \\
\text { couv. }\end{array}$ & $\begin{array}{l}\text { dter Bank } \\
\text { découv. }\end{array}$ & $\begin{array}{l}\text { Rhei } \\
\text { Gre } \\
\text { couv. }\end{array}$ & $\begin{array}{l}\text { nische } \\
\text { dit B. } \\
\text { ádécouv. }\end{array}$ \\
\hline 1894 & 102.3 & 35.4 & 68.3 & 33.9 & 66.7 & 13.2 & 11.2 & 14.9 \\
\hline 1895 & 163.0 & 52.0 & 103.3 & 44.5 & $7 \pi .2$ & 15.4 & 9.5 & 14.1 \\
\hline 1896. & 145.4 & 52.9 & 104.5 & 54.3 & 72.7 & 14.5 & 10.9 & 16.0 \\
\hline 1897. & 172.6 & 63.5 & 124.0 & 69.5 & 68.6 & 18.2 & 12.6 & 21.4 \\
\hline 1898. & 188.6 & 61.7 & 150.0 & 79.3 & 68.7 & 24.2 & 22.5 & 30.8 \\
\hline 1899 . & 219.1 & 76.6 & 163.6 & 85.1 & 69.3 & 17.0 & 28.3 & 35.3 \\
\hline 1900 & 229.8 & 70.2 & 194.2 & 94.6 & 78.2 & 24.2 & 34.7 & 44.2 \\
\hline 1901. & 239.8 & 72.4 & 141.9 & 73.9 & 79.5 & 26.1 & 39.0 & 43.3 \\
\hline
\end{tabular}


forme d'avances à courte échéance pour des inmobilisitlions d'une réalisation difficile. .

La Giazelte de Francfort, pourtant fitrorablu anx grandes banques, le constatait ainsi :

"Toute notre vie économique est basée sur le crédil, si bien qu'on a justement nommé notre époque celle du crédit. Or cette base est anjourd'hui ébranlée. La seconss. actuelle est la conséquence des lautes commises pendant la période précédente. On a acrordé durant ces dernièr's années du erédit à pleines mains sans s’enpuérir toujours suffisamment sul chait justitic. C̈est a ces rédits excessifs quïl laut attribuer l'expansion à quelques rogards malsaine de notre industrie " (1).

Il nest done pas étomnant que la crise industrielle ol commerciale se soit répercutée avec une grande intensité sur les banques elles-mèmes.

Doux banques hypothécaires, la Preussische Hypote kenbank et la Deutsche Grundsehuldbank qui, sortant du cadre d’attributions délimitées par les staluts, ivaient engagé une partie de leur actil dans des sociélés de spirculation, malhonnètement gérérs d’atlleurs, sécroulerent les premieres en novembre 1900. Puis te fut le tour de la Dresdner Credit Anstalt qui avait une grose partic de son capital engagé dans la lompagnic liummer, sociéti d'électricité dresdoise; la chute de la Lresiner Ciredit anstalt détermina celle de la Leipziger lianli, puis celle de

(1) "L'histoire économique est pleine dexemples de ce que des hintques remplies du sentiment de leur puissance ont depasse a la lisen pre les bornes qu'on leur avait postes et, en arcordant trop de cridil, nht

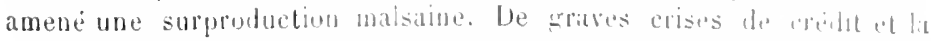

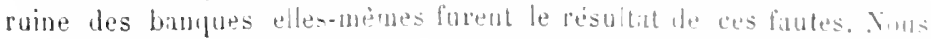

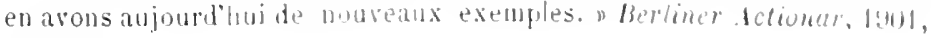
n. $16 \%$. 
la Rheinische Bank, celle de la Sächsische Handelsbank. La crise surprit également la Breslauer Discontobank qui dut abandonner sa maison de Berlin et la Berliner Bank qui avait très largement participé aux émissions et qui subit de très lourdes pertes.

La chute la plus retentissante fut celle de la Leipziger Bank. La Leipziger avait une vieille clientèle et passait pour une des plus solides banques de l'Allemagne centrale ; ses relations avec la Treber Tröclinung de Cassel (Société de séchage des Drèches) causèrent sa perte. Cette société fondée en 1889 avait acquis des brevets pour dessécher les déchets de brasserie et de sucrerie; elle avait réussi à pousser ses actions jusqu'au cours de 1000 0 0 et avait fondé des filiales un peu partout: en Russie, en France (Nantes), en Italie et en Autriche.

Cette eréation de filiales exigeait des capitaux ; en 1896 , la Leipziger Bank onvrait it la Treber Tröcknnng un crédit de 200.000 marks, bientôt porté à 500.000 . A la fin de 1898, la Leipziger Bank se trouvait avoir avancé 28 millions; lors de la faillite de la Treber Tröcknung on parlait d'une créance de 8 à 106 millions de la Leipziger Bank dont les ressources propres atteignaient à peine 50 millions de marks!

La nouvelle de la cessation des paiements à la Leipziger Bank provoqua une véritable panique; les établissements de crédit subirent tous un recul notable; la crise, déjà aggravée par la paralysie des bourses allemandes auxquelles le Börsengesetz de 1896 avait enlevé toute liberté de se mouvoir et toute ćlasticité, s'accentua. L'absence d'une spéculation capable d'agir laissa non seulement tomber les cours au-dessous de la valeur intrinsèque du titre, mais elle rendit mème douteuse la possibilité de vendre nom- 
bre de valeurs. Il en résulta un pessimisme ef mone maliande qui ébranlerent le crédit plus que dre raison dans decirconstances analogues (1). Lin grand nombre dh banpous

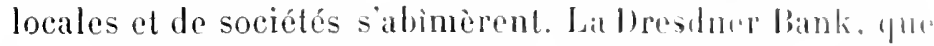
l'on supposait engagéc dans losaffaires des deux princifaux établissements sinistrés dut ru quelques jours restiluer pour 40 millions de marks de dépòts à vur.

Cependant les grands établissoments résistionent victorieusement; liun deux meme sut tirer parli de la siluation. La Deutsche Bank dont les participations industriclles étaient plus solides que colles de sesconcurrentes, profitit de l'óbranlement produit pour recuoillir nue partio de lat clientibe de la Loipziger Bank. Blargir considérablement son cercle d'action ef sélablir definitivement en Siare.

C'est le 20 juin à 11 heures du matin, que le télégraplue avait apporté a Berlin la nouvelle de la cessation des faicments de la Leipziger Bank, provoquée par l'effondrement de la Dresdner Credit Anslall. Le mime jour, l'agrower Wolff faisait paraitre l'information suivante:

"Berlin, 2: juin 1901.0n nous annoncole la Deutschu" Bank : la Dentsche Bank saisit l'oceasion de la cessation des paiements de deux banyurs imporlantes les principales villes de saxe, pour metle à exécution un projed depulis longtemps formé ; elle va far l'installation d'une suceursale a Leipzig élargir son champ d'activití en saxe. Il lium voir le motif de sa résolution dans le fait yue cotter criat-

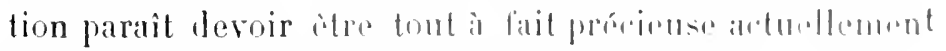

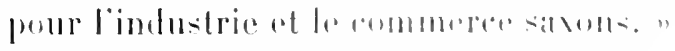

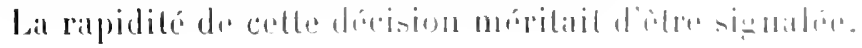

(1) Declarations in Congras des batmpluter ahemands. 
Bientôt après la Deutsche Bank transforme en succursale dresdoise la maison Menz, Blochmann et Cie et s'installe définitivement dans une contrée jusque-là réservée à ses concurrentes. L'année suivante enfin, elle augmente son capital de 10 millions de marks pour absorber la Duisburg-Ruhrorter Bank, une des plus vieilles maisons de la région Rhénane-Westphalienne que la crise avait particulièrement touchée. On voit l'avance prise sur les aulres établissements par la Deutsche Bank moins atteinte par les krachs industriels.

Mais les autres grandes banques berlinoises ne devaient point tarder à se remettre et à tirer également de celte crise mòme des avantages directs et nombreux.

Lattitude courageuse des grands établissements (1), supportant sans faiblir un recul très notable de leurs actions (2), se montrant prêts à satisfaire immédiatement aux exigences de leurs déposants (nous avons vu que la Dresdner Bank dut restituer en quelques jours 40 millions de

(1) Congrès des banquiers, "La crise a été adoucie par l'attitude sensée des grands établissements de crédit, en première ligne par la Deutsche Bank, à l'intervention énergique de laquelle on est redevable du prompt rétablissement de la confiance."

(2) Cours des actions des 6 principales banques berlinoises, au commencement des mois de :

\begin{tabular}{|c|c|c|c|c|c|c|c|c|c|c|c|c|}
\hline & \multicolumn{2}{|c|}{1899} & \multicolumn{2}{|c|}{1900} & \multicolumn{3}{|c|}{1901} & \multicolumn{2}{|c|}{1902} & \multirow{2}{*}{ 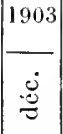 } & \multicolumn{2}{|c|}{190.4} \\
\hline & 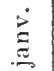 & $\Xi$ & $\stackrel{\equiv}{\equiv}$ & 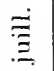 & $\stackrel{\Xi}{\Xi}$ & $\Xi \underset{\vec{u}}{\dot{v}}$ & $\dot{\ddot{\Xi}}$ & $\stackrel{\Xi}{\Xi}$ & $\stackrel{0}{0}$ & & $\Xi$ & ن. \\
\hline Deutsche Bank. . & $20 \mathrm{~s}$ & 210 & 209 & 190 & 197 & 189 & $19 \mathrm{I}$ & 208 & 212 & 224 & 217 & 235 \\
\hline Discontogesells & & & & & & & & & & & & \\
\hline chaft. . . & 198 & 197 & 192 & 174 & 178 & $16 \mathrm{~s}$ & 171 & 181 & 189 & 196 & 185 & 192 \\
\hline Dresdner Bank & 163 & $16 i$ & 164 & $14 x$ & 147 & 116 & 119 & 144 & 144 & 157 & J 4 & 158 \\
\hline Darmstädter $B$. & 155 & 152 & 145 & 134 & 134 & 111 & 114 & 137 & 13] & 146 & 136 & 342 \\
\hline Berliner Hand. & $16 \mathrm{~s}$ & 172 & 169 & $1 \div 6$ & 152 & 129 & 131 & 155 & $15 \mathrm{~s}$ & 163 & 151 & 165 \\
\hline National Bank. & $1+8$ & $1 \div 6$ & $1: 6$ & $13 i$ & 133 & 96 & $9 s$ & 1) 4 & 117 & 127 & 120 & 130 \\
\hline
\end{tabular}


marks de dépòts). la rapiditi de la reprise (les allition- (1)

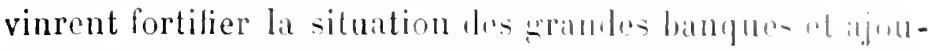

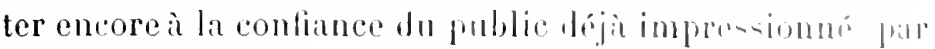
l'annonce des capitaux considérablesed des allsmontations le capital.

Dautre part la liallite de nombrenses bingues locales avait prouvé une fois de plus combien il rebit diungerens de saventurer dans les opréations financierrs. phand nest point assurée, par la diffusion mome dos alfailns. mun tros large compensation des risques. Lal epise entin arat chide laveu de tous, ageravée pal les eflets de la loi sur les bourses: les banques de province ne sétaicht point fait inserire au registre de Bourso et linsécurité qui ron résultait était apparue plus charement.

Toutes ces raisons contribuierent it rapprocher le public des grandes banques (2).

(1) Pour cent des dividendes distribués de ln99 a lon't a la

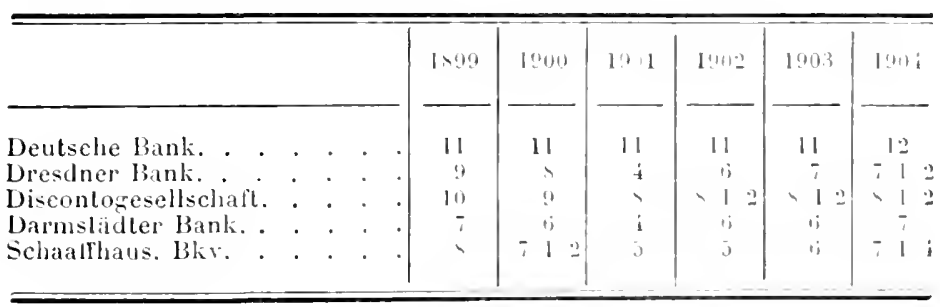

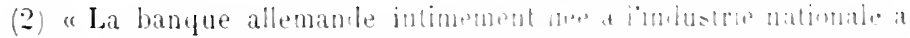

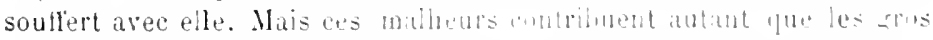

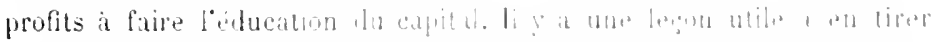

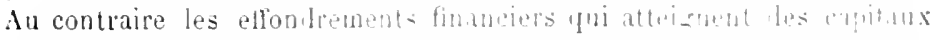

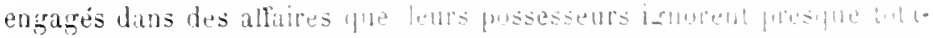

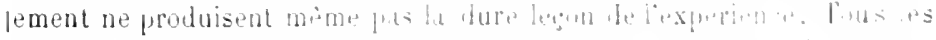

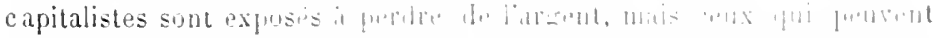

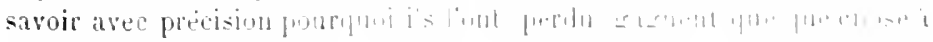

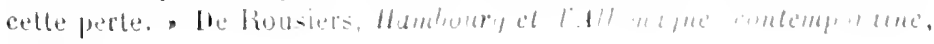
p. St. 
Mais surtout il faut noter l'élan que vint donner au mouvement de concentration l'exemple de la Dentsche Bank. Les grandes banques berlinoises ne voulurent point laisser leur grande concurrente conserver cette avance et s'efforcèrent de regagner le temps et le chemin perdus. Aussi bien, la crise venait de leur faire comprendre à quels dangers clles s'exposaient en immobilisant trop leurs ressources et la nécessité de diriger leur activité, plus que par le passé, vers les opérations ordinaires de banque.

La Discontogesellschaft qui jusque-là avait paru se désintéresser de ce mouvement d'expansion à travers les provinces et qui ne comptait encore en 1900 que 1 succursale et 3 participations on commandites, multiplie à partir de cette date ses participations et ses absorplions, notamment sur le Rhin (Rheinische Discontogesellschaft, Bank für Rheinland and Westialen, Coblenzer Bank) et en Saxe (alliance avec l'Allgemeine Deutsehe Credit Anstalt, absorption de la banque Günther et Rudolph de Dresde), les deux contrées particulièrement louchées par la erise.

La Darmstädter Bank reprend le siège berlinois de la Breslauer Discontobank fortement ébranlée par la chute de la Leipriger Bank, absorbe à Mannhein la Bank für Süddeutschlanel et pénètre dans le Hanovre par l'absorption de la banque Oppenheimer et Davisor.

La Berliner Bank ne se remettra pas de la crise; une première tentative de fusion avec la Deutsehe Bank n'aboutit pas; elle sera absorbée en janvier 1908 par la jeune et active Commerz und Disconto Bank.

"Ces fusions de grand style, écrivait le Berliner Actionür le 9 août 1902, au contraire des années précédentes, se sont faites sous l'empire de graves circoustances et ont 
prouvé quavee le temps la vie des banques provinabalos était devenue phus difficile en présence des prandes lanques. C'est ce qui explique encore la lusion tonte récentu de l'Essener Credit Anstalt avee la Roeklinghauser liank " (groupe Deutsche Bank).

La Dresdner Bank enfin, voulant dépasser d'un seul coup ses rivales. conchut avee l'A. Schatifhatusens'cher' Bankverein une formidable association d"intercits 1). Lat Deutsche Bank riposte aussitót en portant son citpital à 180 millions de marks, la Discontogerseltschalt porte le sien a 170 millions, la Darmstidter bank a lö millions. Jamais la lutte d’intluence et de capitaux n’a cté aussi vive qu'en ces dernières annóes.

Ainsi done, la crise 1900-1901 a singulièrement contribué à cetle accélération du mouvenent de concentration en poussant les grandes banques. un peu par amour-propre, nous disait-on, beancoup lar nécessité, átendre le champ de leur activité pour augmenter le courant de leurs opérations de banque proprement dites, siassurer une plus large compensation de's risques, soutenir it nouveiu les industries nationales ibrinlées et lene proenrer de nouveaux débouchés, comme aussi hien en dietachant le. public des petites banques que leur rayon d'aclion plus restreint et de moindres garantirs apparentes meltent de moins en moins à mème de reerureillir les capitaux llottants et d'en trouver l'emploi.

(1) Voir au chapitre suirant l'atule de cetle association d'interits at les nombreuses absorptions qui l'unt suivie. 


\section{CHAPITRE III}

\section{LES MOYENS TECHNIQUES .}

LES MODALITÉS DU MOUVEMENT DE CONCENTRATION

La concentration dans le trafic de banque en Allemagne s'est opérée, avons-nous dit, d'une façon particulièrement originale; elle est loin de présenter les mèmes formes qu'en France ou en Angleterre par exemple. Si le but poursuivi reste le mème, - drainer, pomper partout les capilaux isolés, trouver de nouveaux emplois, - si le moyen général, peut-on dire, ne change pas, - assurer la diffusion de l'établissement, lui procurer en quelque sorte l'ubiquité, - les voies et les méthorles diffèrent profondément.

Indiquons, dès maintenant, les principales modalités que revêt en Allemagne le mouvement de concentration dans le tralic de banque.

10 Tout d'abord les succursales proprement dites des grandes banques allemandes sont peu nombreuses et proviennent généralement de fusions ou d'absorptions totales.

$2^{\circ}$ Un second mode de concentration consiste dans la transformation, par les grands établissements, des firmes privées en socictés par actions.

$33^{\circ}$ La commandile de banques secondaires ou les participations prises chez elles parr les grands établissements constituent deux procédés très couramment employés. 
$4^{\circ}$ Entin nous nous trouvons en prósonere the A suckete

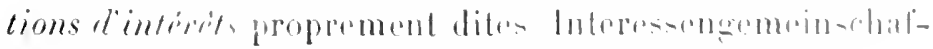
ten) préparérs par les consontiume de banques (1.

\section{$1^{\circ}$ Succursalos.}

Les hanques anglaises ol mos grandes sociétís flançations ont surtout pratigur la politique deremsiom dierese par

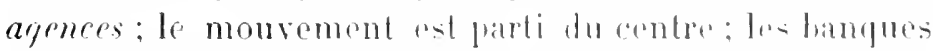

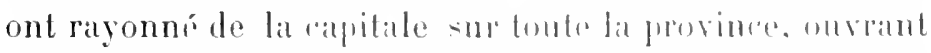
sur les differentes platers - en rommenciant par les villes les plus importantes pour linir parde moindres focalitios - des agenees. bureatux el suremrales qui vinent roncurrencer directement les bampuine locams. Les banques

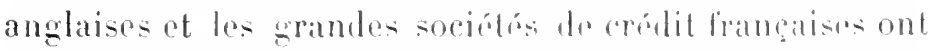

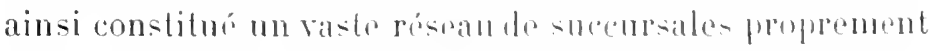
dites, dont les tableanx suivants montrent l'itemdue:

\section{sucrusales, ayences et buremur an 2 I decembie l!)11'.}

\begin{tabular}{|c|c|c|c|c|c|}
\hline Credit Lyonnais. . . . . . . & $\begin{array}{c}\text { P'aris } \\
3 ! !\end{array}$ & lant. & $\begin{array}{c}\text { Province } \\
\text { liis }\end{array}$ & $\begin{array}{l}\text { Etranzer } \\
1 \%\end{array}$ & $\begin{array}{l}\text { Tolil } \\
2: 33\end{array}$ \\
\hline Comptoir National d'liscomple. & $2 ! !$ & $!$ & 110 & 292 & 171 \\
\hline Société Générale. . . . . & $\because 3$ & 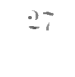 & $1: 2 \cdot 2$ & $\begin{array}{l}1 \text { agence } \\
+3 \text { tiliales }\end{array}$ & $i 3$ \\
\hline & 113 & i:i & 7010 & 43 & $9+01$ \\
\hline
\end{tabular}

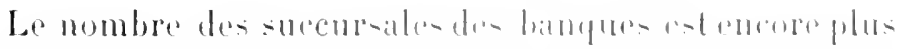

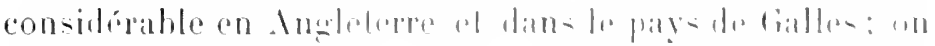

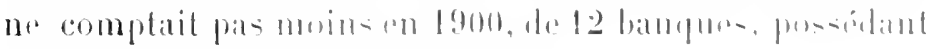

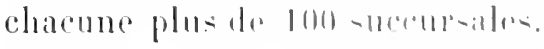

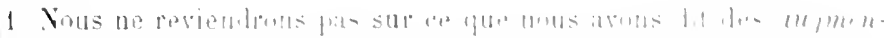

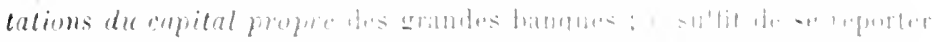

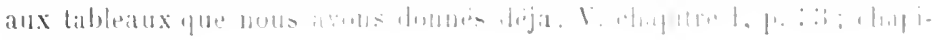
tre II, p. . . 
London City and Midland. 283

Lloyds . . . . . . 278

Capital and Counly. . . 269

Barclay and Co..... 241

National Provincial. . . 240

London and Provincial . . 189
London and County . . 185

Metropolitan. . . . . 133

Wilts and Dorset. . . . 128

London and South Western 127

Parl's Bank. . . . . 117

York City and County. . . 102

\section{Soit un total de 2.292 succursales (1).}

En Ecosse, ce nombre est encore, proportionnellement à la population, pius élevé : 11 banques et 1.043 succursales pour un peu plus de deux millions et demi d'habitants.

En Allemagne, le nombre des succursales proprement dites possédées par les grands établissements est incomparablement moins élevé et encore la fondation de la plupart de ces succursales, caisses des dépôts et aggences ne romonte guère au-delà de ces dernières années comme il est aisé de s'en rendre compte par la comparaison suivante:

Tableau des succursales, caisses de dépôts et agences au début des années:

\begin{tabular}{|c|c|c|c|c|c|c|}
\hline & \multicolumn{3}{|c|}{$189 \%$} & \multicolumn{3}{|c|}{$1900(\mathbf{2})$} \\
\hline & Succursales & $\begin{array}{l}\text { Caisses } \\
\text { de dépồts }\end{array}$ & Tolit & Succursales & $\begin{array}{l}\text { Caisses } \\
\text { de dépôts }\end{array}$ & Tolal \\
\hline Deutsche Bank. . . . & 4 & 12 & 16 & 4 & $1 \tau$ & 21 \\
\hline Dresdner Banls . . . . . & 3 & 3 & 6 & 9 & 17 & 26 \\
\hline l)iscontogesellsclraft. . & 1 & 1 & 2 & 1 & 1 & 2 \\
\hline Darmstälter . . . . . & 2 & 1 & 3 & 2 & 4 & 6 \\
\hline Schaaffhaus. Bkv . . . & 1 & - & 1 & 1 & 1 & 2 \\
\hline $\begin{array}{l}\text { National liank. } \cdot \dot{\cdot} \cdot \\
\text { Commerz und Disconto }\end{array}$ & - & 9 & 9 & - & 11 & 11 \\
\hline Bank. . . . . . & - & - & - & 2 & 2 & 4 \\
\hline Milleldeutsche Credit B. & 2 & 3 & $\ddot{3}$ & 2 & 10 & 12 \\
\hline & & 29 & 42 & 21 & 63 & \\
\hline
\end{tabular}

1. En 1901, 21 banques possédaient plus de 100 filiales chacune. An dibut de 1905, la seule London City et Widdlan Bank comptait $4 \mathbf{4 7}$ filiales. En 1905 on compte $\$ 45$ succursales de banques.

:2) A cette mème date nos 3 grandes sociélés de crédit possédaient: 
Tablean des sucenisales, raisises de dipgits al ugences an 31 derembir l!n

\begin{tabular}{|c|c|c|c|c|c|c|}
\hline & & & Cairses & de depoits & & \\
\hline & & Filiales & Berlin & Province & Etranger & Total \\
\hline Deutsche Rank... & . & . $\quad \tau$ & $2 \tau$ & 21 & 1 & :it \\
\hline Discontogesellschaft. . & & 4 & 7 & $\because$ & 1 & 1:t \\
\hline Dresdner Bank. . . . & & 1't & 18 & 10 & 1 & 13 \\
\hline A. Schaaffhaus. Bkv. . & & 10 & .3 & 10 & - & $\sim$. \\
\hline Darmstïdter li. . . . & & i & 11 & $\therefore$ & - & \\
\hline Mitteldeutsche Cred. B & & i & l11 & 6 & - & \\
\hline National li...... & & - & $1 ' t$ & - & - & \\
\hline Commerz u. Disconto B & & 2 & $1: i$ & 10 & - & \\
\hline & & $4:$ & $10 \%$ & 13.3 & 3 & \\
\hline
\end{tabular}

Deutsehe Bank, à Berlin. Filiales à Brime, Mreste. Francfort, Ilambours. Loiprig. Lomber, Bunich. Nïrembere.

Discontogespllscha/t. Filiales a Berlin. Franchort. Brime. Londres, Ilamboure (Norddeutselue liank aree 2 adisses de dépots a Altona ot Marturon.

Dreselmor Banti Filiales à Berlin, Drestle, Lomdres. Francfort, Brime, Birckebure, Chomnite, Fiontl, Ilam-

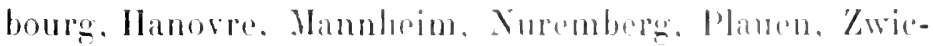
kial. Munich.

Schaaffh. Bher Filiales à Berlin, Hïscldorl. Bonn, Juis-

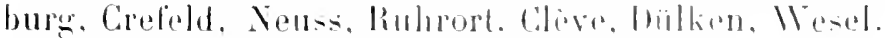

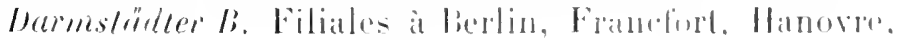
Sitrabloure.

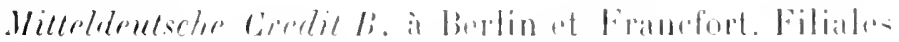
a Meiningen, Mir-batron, Fïrth. Norrombers.

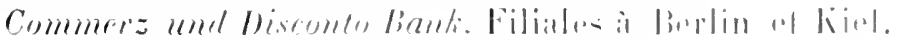

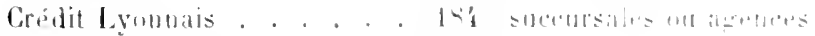

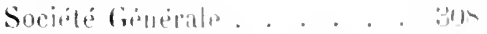

Comptuir National d'Escomptos. 1:21 
On voit d'après ce tableau, que les grands établissements berlinois ne possèdent de filiales que dans les centres tout à fait importanls. Les caisses de dépòts et les bureaux de change (Deposilenkassen, Wechselstuben) ne se rencontrent guère également que dans les grandes villes: les 48 Depositenkassen de le Deutsche Bank par exemple, se réparlissent ainsi : 27 à Berlin, ö à Dresde, 10 à Hambourg, $ّ$ à Leipzig, 1 a Wiesbaden. Files remplissent les meimes lonctions que les bureaux de quartier des sociétés de crédit françaises (dépòt et retrait des fonds, payement des coupons, garde des litres, etc.). Leur développement en ces derniòres années, dù à l'augmentation des affaires de banque proprement dites dans les grands établissements, nolfre rien qui puisse nous arrèter particulièrement.

Les filinles (succursales), elles, présentent des traits originaux qui les font différer profondément des succursales du Crédit Lyonnais on de la Société Générale, au double point de vue de leur fondation et de leur fonctionnement.

a) Elles ne sont point, en effet, lo plus souvent, créées de toutes pièces et n'apparaissent point, un beau jour, sur une place, firmes nouvelles, sans allacloes et sans passé. Elles naissent en général, de l’absorption totale d'une maison privéc dont le grand itablissement reprend tout l'actif ol lout le passif, ou de la fusion complète d'une banque par actions avee le grand établissement qui désormais en détiendra la tolalité des actions. C'est lit, la polilique d'amalgamalion, comme disent les Inglais, opposée à la politique d'extension directe par agences (1). Les grandes

(1) En France, les exemples l'absorptions ne se rencontrent guère 


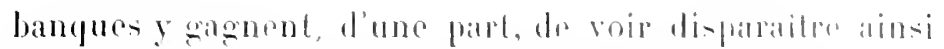

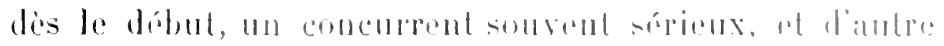
part, trourent à cette combination ant immense arantign qu'elle leur procure d'ores et dígit un noyan de clientibe tout formé. Sins compter que parforis te chef de lat banquer locale ainsi fusionnée reste it lit libte le sa maison transformée en filiale et que sil connatisillee des hommes et des besoins locaux est mu précieux gage de suceios pour l'arenir de la suceursale.

La plupart des filiales des grande banques berliuoises ont été créées de cette firgon:

Deutsche Bank: i filiates on Mllemagne: :B résultant d'alsorptions: Frandiort aborption du Frankfuricr biankverein, 1886), Leipzig reprice les alfaires de la Leipziger Bank, 1901), Dresde (absorption de la maison Menz, Blochmann et Cie, 1901).

Drestner Bank: 14 filiales: 7 absorplions: 1892, Din Anglo Deutsche Bank, Ilambours; 1898 , Bremor Bank,

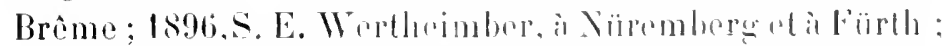
1898. Alexandre Simon, it Hanorre. W. I. Cintmann, is

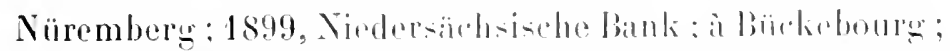

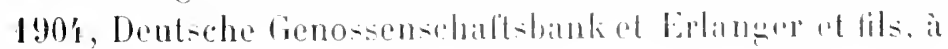
Francforl.

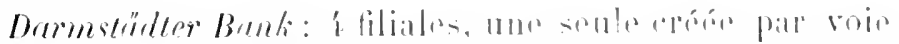

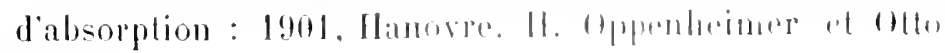
Davison.

Discontegesellselate: i liliales. trois far als-orplions:

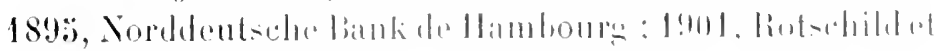

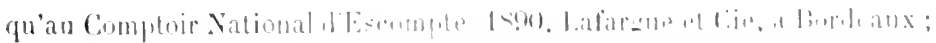

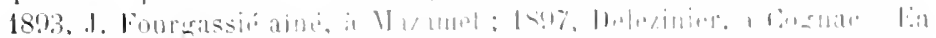

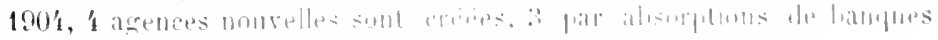

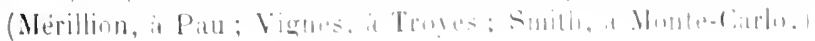


fils, à Francfort; 1904, J. Schultze et Wolde, à Brême. Voici d'ailleurs le tableau général du nombre de ban. ques privées et de banques par actions absorbées par les 4 principaux groupes (août 1905).

\begin{tabular}{|c|c|c|c|}
\hline & $\begin{array}{l}\text { Banques } \\
\text { privées }\end{array}$ & $\begin{array}{c}\text { Banques } \\
\text { par actions }\end{array}$ & Total \\
\hline Deutsche Bank . . . . & 31 & 12 & 43 \\
\hline Dresdner-Schaaffhaus. . . . & 27 & 14 & 41 \\
\hline Discontogesellschaft. . . . & 17 & 11 & 28 \\
\hline Darmstädter . . . . . & 13 & 3 & 16 \\
\hline
\end{tabular}

L'exemple le plus fameux dans cet ordre d'idées est celui de l'absorption de la Norddeutsche Bank de Hambourg par la Discontogesellschaft en 189.5 . Les relations des deux banques remontaient à 1871 ; plus tard, toutes deux s'engagèrent dans la création des chemins de fer du Vénézuela, de la Compagnie internationale d’air comprimé et d'électricité $\mathrm{Popp}_{\mathrm{p}}$; les pertes qu'éprouva simultanément de ces deux côtés la Norddeutsche Bank, déterminèrent sa fusion avec la Disconto également touchée l'ailleurs. Par décision de son assemblée générale du 16 avpil 189 ö, la Norddeutsche Bank transmit à la Discontogesellschaft son passif, son actif et sa raison sociale.

Cette dernière, par statuts du 16 avril 1895, transforma la Norddentsche Bank en une société en commandite par actions dont elle garde, à elle seule, le capital-actions, 40 millions de marks.

b) De fait la Norddeutsche Bank est devenue une simple filiale de la Disconto, mais - et ceci nous permet de bien saisir la seconde différence caractéristique des filiales des grandes banques berlinoises - elle a gardé une autonomie formelle et une très large indépendance. Il n'est pas rare de voir les filiales conserver le nom et la raison 
sociale des banques locales qu'elles ont remplacées; lin lbremer Bank, la Niedersibshische Bank sont de pures liliales de la Dresdner Bank comme la Remscheider Bank, pour emprunter quelques exemples aux hanques secondaires, est une succursale de la Bergisch-Markische Bank, la Schalker Bank, la Credit Bank dr Recklinghausen, des suceursales de l'Essener Credit Anstalt.

Sans ètre toujours aussi distinctes lu siège central que l'est la Norddeutsche Bank, les filiales allemandes ont en général une plus grante importance que les agences du Crédit Lyonnais ou du Comptoir National d'Escompte. Investies à peu près des mìmes droits que le siège central, elles sont en rapports moin - etroits aver lui, sontipessent parfois à des opérations auxquelles il ne participe point et ont souvent leurs affaires propres, suivant l’industrie ou le commerce des régions où elles sont établies, voire quand il s'agit d'emprunts de villes ou de petits Etats (1) Le directeur dime des suecursales de la Deutsche Banli nous disait à quel point les grands établissements tiennent à assurer cette indépendance et cette libertéd atction des filiales: "Les filiales ainsi comprises, disait-il, n'atmènent point une lourde sureharge de frais généraux: point de contròle incessant et máliculeus, point d'ordres de détails; nous connairons la ligne de comblute générale à suivre et me affaire nouvello une fois autorisice en principe, nous la gérons pour le mienx et suivint les né-

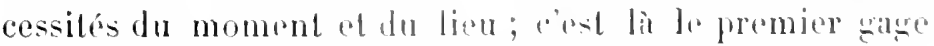
d'une bonne adaptation anx besoins de lat chientiele."

Nous nous trouvons donc, dis les filiales proprement

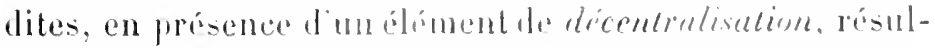

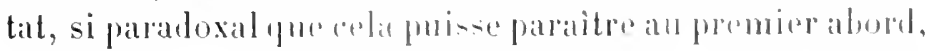

(1) V. des exemples an tableau des partivipations te la Deutsche Bank (1903-190' , Ho partie, chap. IlI. 
de la concentration elle-mème. Il vá d'ailleurs nous apparaitre, plus fortenent marqué encore, dans l'examen des autres modalités qu'a revètu le mouvement de concentration des banques en Allemagne.

Co: n'est point, en effet, le nombre des succursales proprement dites qui limile l'étendue du rayon d'action des grandes banques berlinoises: leur sphère d"influence est infiniment plus vaste. Partout où elles n'ont point établi de filiales, elles vont entrer en contact avec le public et la clientèle au moyen de correspondants plus ou moins attitrés, plus ou moins indépendants, mais qui, ayant encore plus d'initiative qu'une pure succursale, vont pouvoir seconder plus eflicacement la hardiesse de la direction centrale.

Ce second aspect du mouvement de concentration est particulièrement original et tout à fait propre à l'Allemagne; il mérite d’ètre étudié avec quelques détails.

\section{$2^{\circ}$ Commandites.}

Un premier moyen plus souple que celui de l'établissement de succursales propres, consiste, pour le grand établissement désireux de s'installer sur une place déterminée, à simplement commanditer une banque particulière de cette place. Outre que la banque commanditaire aide ainsi puisamment à l'extension de la banque commanditée en venant augmenter ses moyens d'action, elle assure ses propres intérèts sur la place par une représentation active de ses capitaux, le plus souvent aussi par l'entrée d'un de ses directeurs dans la banque secondaire; elle atteint ainsi le même but que par l'installation d'une succursale. 
L'exemple le plus important de er mode de conentration, est sans contredit celui de la commandite de la maison R. Varschauer de Berlin par la Damatüller banti pone 20 millions de marks ( 1898 , (1). Nous atrons dijit cilé cet exemple lors de la discussion des causes particulieres. Que des motifs purement prives aient pu décider M. R. Varschauer à aecepter la combinaion proposie par la Darmstädter Bank, cela nous intérese en somme, assez peu ; ce qui est plus important à nos peux, ciest le procedé employé par la Darmstälter Bank fue la concentralion eroissante des alfaires de banque à bertin obligrail a fonder une suecursalo dans la capitale, pour réaliser ce hut el, comme le dit tres bien 11 . Sayous, "faire bonne figure dans la capitale, sadapter au milieu dans la mesure du possible, sans soulever cependant lesprotestations de lancienne clientèle ot des actionnaires provinciaux. "

Les grandes banques berlinoises et les banque- provinciales elles-mèmes ont eu souvent recours à ce procédé d'amalgamation, incomplete sans dontr, malis lriesullisante pour élargir le champ de la compensation, donner ì la banque commanditaire la haute main dans les atlaires de la maison commanditée pui, de phus, preste autonome, plus indépendante, mieux appropriécax busins de la plientiole locale, tout en amenant it l'álibli-nement directeur un nouvel et important conrant dallaires ef an lui ourrant de nouveaux débouchús.

Voici le tableat des hamplues rommanditions par les

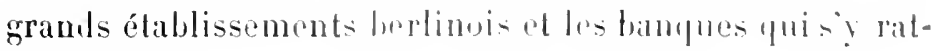
tachent.

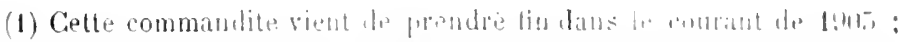

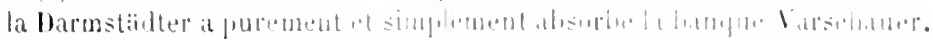

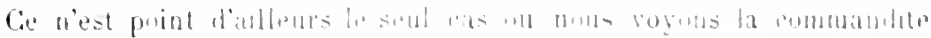
préparer, pour ainsi dire. les roles at la fustosia totale. 
Deutsche Bank.

Bergisch Märkische Bank.

Schlesischer Bankverein.

Osnabrücker Bank.

Hildesheimer Bank.

Essener Credit Anstalt.

Rheinische Credit Bank.

Disconto Gesellschaft.

Norddeutsche Bank.

Allgemeine Deutsche Credit Anstalt.

Süddeutsche Discontog.

Dresduer Bank.

Schaaffausens'cher BKv.

Darmstædter Bank.

Breslauer Disconto Bank.
Commandites .

C. E. Heydemann (Bautzen). Guillermo Vogel (Madrid). Rosenfeld et $\mathrm{C}^{\circ}$ (Vienne). Hüser et Co (Solingen). Max Gerson et Co (Hamm). D. Fleck et Scheuer (Düsseldorf). G. Fromberg el Co (Berlin). R. Vogt et $\Gamma^{\circ}$ (Frankenstein). H. C. Weiss et $\mathrm{C}^{\circ}$ (Königshülte). Quakenbrücker Bank. Marcus Ganz (Herford).

L. Peters Nachf (Braunscbweig) Benfey et $\mathrm{C}^{\circ}$ (Göttigen).

E. Osthaüs (Hagen).

C. Basse (Lüdenscheid).

J. Cahn et $\mathrm{C}^{0}$ (Pforzheim).

G. Grohé Heinrich et $\mathrm{C}^{\circ}$ (Saarbrücken).

B. Burger et $\mathrm{C}^{\circ}$ (Wolfach).

E. Tornsquist (Buenos-Ayres).

A. de Bary et C (Anvers).

E. Meyer (llanovre).

Heydemann (Löbau).

E. Ladenburg (Francfort). Bosse Keil et $\mathrm{C}^{0}$ (Berlin).

P. Elimeyer (Dresde).

11. Arnhold et $\mathrm{C}^{0}$ (Hall). Loeb el Co (Neuestadt). Rümelin et $\mathrm{C}^{\circ}$ (Heilbronn).

Schmitz Heidelberger et $\mathrm{C}^{\circ}$ (Mainz).

Wingenroth, Soherr et $\mathrm{C}^{\circ}$ (Mannheim).

A. Kuntze et Co (Dresde). Fuld et $\mathrm{C}_{0}$ (Pforzeim).

Oppenheim und Schweitze. (Breslau).

Fliesbachs Wwe (Glogau).

(Gruneberg). 
Obstbank fiur llandel u. liewerhe.

Mitteldeutsche Credit Bank.

National Bank.

Commerz und Disconto Bank
R. Uamme bantzim .

Meyer el Dis, Baden-liatan

North liammeier ef $C^{\circ} \mathrm{E}$ :-ent

Erltel, Freybers el Co lleiprigr

. Dreyfus el Co ltrantonl.

Credil und spar Bank Leipzig).

Soil un total de 41 banques commanditées prar les zrands Instituts berlinois et les banques qui iy rattuhent; on no. considérant pour le moment que les banques berhinoises nous nous trouvons en présence de 19 banques comnanditées. premier et déjia appréciable résultat t'expansion indirecte, pent-on dire.

\section{$3^{\circ}$ Participations.}

Mais beaucoup plus nombreuses sont les participations prises par les banques berlinoises dans les banques par actions provinciales, commandites partieulieres que nous voyons se réaliser par achat ou par b́change d'une partie des actions, avec ou sans conversion. alvec ou sans le consentement de la banque ainsi commanditée. Coest la le procédé caractéristique par excellence du mouvenunt de concentration dans les banques allemandes, le moven par lequel s’est réalisée la constitution de ces grompes de bunques, dont nous avons donné le tablealu.

Il nous faut maintenant domer puelyus exemples pho détaillés et préciser les arantages de ce procídé.

a) Partois le grand établi-sement concourt à la transformation premiore diune hanque privie en une soritite far actions; il détiendral la majenre partie du capilat-actions et aura de cette façon la hamte direction dans les allatires du nouvel établisiement: los deux banques dáb. 
guent mutuellement un ou plusieurs de leurs directeurs dans chacun des deux conseils d'administration pour assurer ainsi l'unité de vues. C'est ce que nous voyons réalisé par exemple dans la transformation par la Discontogesellschaft de la maison W. H. Ladenburg, de Mannheim, en Sïddeutsche Discontogesellsehaft.

b) Plus souvent, le grand établissement assure sa participation dans une banque par actions par l'acquisition ferme d'une part importante des actions. Dans cette acquisition libre, it n'est même pas besoin de demander le consentement de la banque commanditée à la participation de sa concurrente plus forte en capilaux.

C'est ainsi que la Deutsche Bank acquit pour 200.000 marks d'actions de l'Essener Credit Anstalt.

c) Plus fréquemment encore, les participations se produisent par échange réciproque des actions des deux banques; le lien est ainsi bilatéral el la communauté d'intérêts plus étroite et plus forte. Les exemples ici sont nombreux et précis; nous rapporterons un des plus caractéristiques, celui de la participalion de la Deutsche Bank dans l'activité de ses deux grandes concurrentes, l'une à l'Est, l'autre à l'Ouest de l'Allemagne, la Bergisch Märkische Bank et le Schlesischer Banliverein.

Le brillant essor de l'industrie minière qui suivit la période 1890-1895, avait reporté l'attention de la Deutsche Banksur lescentres houillers. Désireuse de participer à cette activité d'une façon plus immédiate et d'avoir dès correspondants en titre sur les lieux mêmes, clle proposa d'une part ì la Bergisch Märkische Bank, d'autre part au Schlesischer Bankverein une alliance dont le rapport gé- 
néral de la Deutsche Bank de 1897 prócise aln-1 lan motifs el les bases: "Lat concentration croissante qui diaji al amené un certain nombre d'établissements provincianx ì eréer des succursales à Berlin, nous montre qu'il eol nócessaire de resserrer nos liens avec la province. Ces pourquoi nous avons fait aux actionnaires de la Berewisch llarkische Bank et du Schlesischer Bankverein, qui poursuivent à peu près le mème genrad'alfaires que nous et ont le mème programme, la proposition d'un íchange de leurs actions contre de nouvelles actions de la Deutsche Bank à créer. Cette proposition fut acceptée par plus des 3,1 ides actionnaires. Ainsi l lindépendance des deux banques n’est atteinte en aucune façon."

En 1897, la Bergisch Märkische Bank représentait un eapital de 40 millions de marks, le Schlesischer Bankverein, un capital de 27 millions, soit au total 67 millious plus 1 ö millions ö de réserves. La Deutsche bank aug. menta son propre capital de $\ddot{0} 0$ millions et offrit aux actionnaires de la Bergisch Mạrkische et lu Schlesischer Bankverein de leur échanger, du 28 aoùt au ls septembre 1897, leurs actions contre de nouvellesations le lil Deutsche Bank dans la proportion suirante: ") actions le la liergisch Märkische contre 4 nouvelles ; 3 du Schlısischul Bankverein contre 2. Ainsi, $6.000 \mathrm{mar}$ - dietions the la Bergisch Märkische donnaient droit à h.s00 marlis de nouvelles actions de la Deutsche Bank; 1.800 marlis d'actions du Sehlesischer Bankrerein donnaicnt droit à 1.200 marks de nouvelles actions de lat Deutsche bink.

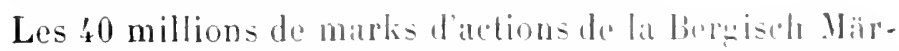

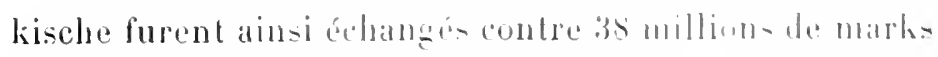
des nouvelles actions de la Deutsche biali ; Les 27 millions 
de marks d'actions du Schlesischer Bankvercin contre 12 millions de marks.

L'année suivante, le rapport général de la Deutselıe Bank s'exprimait ainsi : "Nos relations avec la Bergisch Märkische Bank et le Schlesischer Bankverein se sont confirmées par un résultat aussi favorable pour ces maisons que pour nous-mêmes. La communauté d'intérêts ainsi créée a permis d'assurer à la clientèle de ces deux établissements, de plus grands avantages grâce à l'amélioration des informations et à une facilité plus grande de combiner leurs affaires. Cela gràce à l'aide complaisante de plusieurs directions de banques qu'ils ont maintenant à leur disposition... Nous croyons devoir persister dans cette ligne de conduite. Aussi, nous avons conclu cette année de nouveaux arrangements avec la Hannoversche Bank et avec l'Oberrheinische Bank, à la suite desquels nous avons acquis un montanl d'actions de ces banques, en réalité peu élevé, mais avec l'intention de le conserver solidement pour nous créer ainsi des rapports plus durables en vuc d'aboutir ì un principe fédéral."

De son côté le rapport de la Bergisch Märkische Bank disait: "Les raisons qui nous ont poussé à conclure cet accord avec la Deutsche Bank se résument en ceci : cette combinaison laisse intactes notre indépendance et notre organisation; elle ne nous fait point nous détourner de notre vrai terrain d'action par la préoccupation de fonder une filiale dans la capitale. "

La Deutsche Bank a été moins heureuse dans sa tentative d'absorption de la Berliner Bank. Il peut se faire en effel, que les actionnaires de la banque que le grand établissement désire contrôler se refusent à souscrire aux propositions faites. En mai 1903 , le principe d'une fusion 


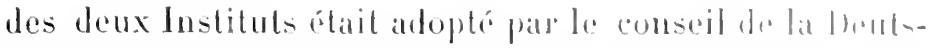
che Bank et celui de ha Berlimer Bank, sous rinerve bien

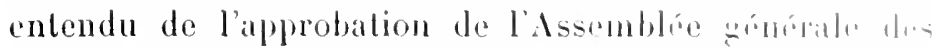
actionnaires. Lat beutsche Bank avait, dans centhe intention, annoncé une élévation de capital de 20 millions dr. matris. Mais l’assemblée des actionnares de la berliner liank. convoquée pour le 31 mai, ne put se constiture faute du quorum nécessaire; alle ne put réunir que 르, s millions

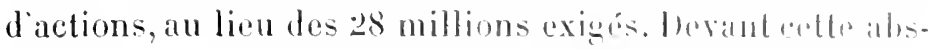
tention qui fut consideré comme une oppositim in pros. jet, la Deutsche bank retira sal pupestion, mais nom maintint pas moins son chation de capital.

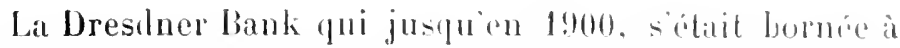
absorber purement et simplement les banquess serondiares a suivi depuis, lexemple de la lleutsole bianli el clle a allquis un certain nombre d'atetions de hanques provinciates quelle porte à son bilan sous le titre de "participations durables ". La biscontogesellschaft et la Bank fïr lland..l und lndustrie ont généralement procédé de ceette mirme fa-

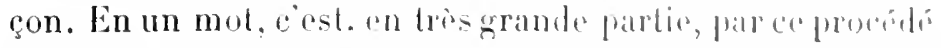
d'achat ou d'échangedes actions que se somt formós lies

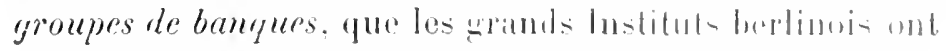

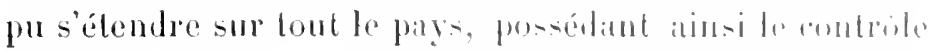
el la direction d'un nombe plus ou moino gramel de banques alliées, unies entre elle's et qui tont an combervalnt

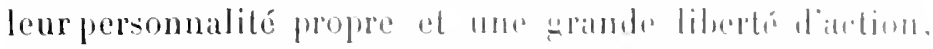

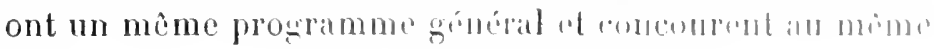
but.

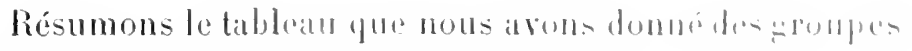
de banques:

La beutsche Benete posside l:3 participations al i partici- 
pations de seconde main, c'est-à-dire les participations propres de ces banques directement unies, rattachées à elle.

La Diesdner Bants 8 participations et 2 sous-participations.

La Disconlogesellschatt 6

La Durmstälter $B$. 4

L'A. Schaaffstaus. B. 3

$\begin{array}{lll}\gg & 9 & \\ " & 1 & \\ " & \frac{1}{18} & \end{array}$

De ces 52 participations possédées par les grands Institutsberlinois, - et ceci nous montreril mieux encore l'esprit qui guida leur politique d'extension, - 22, représentant un capital de plus de 482 millions de marks sont situées dans les districts industriels de la Silésie et de la Prusse Rhénane.

En voici le tableau :

$$
\text { 1. - Parlicipations en Silésie. }
$$

1. Deutsche Bank.

Schlesischer Bankverein. Breslau . . 30.000.000

Il. Dresdner Bank.

Oberschlesische Bank. Beuthen. . $\quad 3.000 .000$

III. Darmstædter Bank.

Breslauer Disconto Bank. Breslau . . 2000.000

\section{1. - Région Rhénane-Westphalienne.}

\section{Deutsche Bank.}

Bergisch Märkische Bank. Duisburg liuhrorter Bank.

Elberfeld. . 60.000 .000 Essener Credit Anstalt. Westfälischer Bankverein. Siegener Rank.

Duisburg. . . 12.000 .000

Essen. . . 36.000 .000

Milnster . . 6.000 .000

Essener Banliverein.

Siegen . . 4.000 .000

Essen . . $\frac{7.500 .000}{125.500 .000}$ 
I1. Discontogesellschaft.

Rheinische Discontogesellsch.

Bochumer Bank.

Eschweiler Bank.

Dïrener liank.

Oberbergische Bank.

Fupener Credit liank.

Barmer Bankverein.

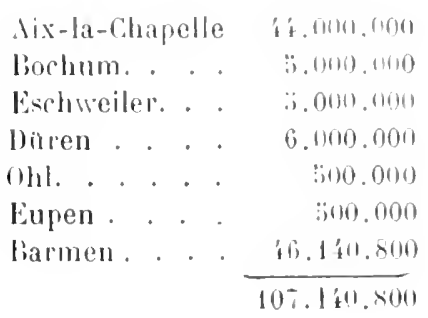

III. Dresdner-Schaaffhausens cher Bkv.

Schaaflrausens cher liankerein.

Mirkische Bank.

Cologne. . . 12:0000.1000

Rheinische Bank.

liwrlum. . . 8.000.000

Ilülheimer Bank.

Viillıeim. . . 10.000.000

Westliilische Lippische Vereinsbank. Pielefeld. . .

Wittelrheinische bank.

Coblenz. . $\frac{12.000 .000}{107.300 .001}$

$107.500 .0(10)$

Cette façon de procéder par voic d"alssorptions incomplites présente de trís grands arantages. ()utro crus que nous avons eu dejà loceasion de sigmaler (adaptalion phus fitcile an milieu, novau do clientide, eonnabsance des hommes et des hesoins locaux), whe nocessite des dreplacements de capilaux bien moins comsidralubs, mosurveillance et un contròle beaucoup moins atroils ot par conséquent moins coûtrux.

De plus, les grands Instituls burlinois sosml ainsi assurés ì bon compte, non seulemont le rombrib des birn-

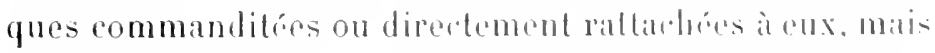

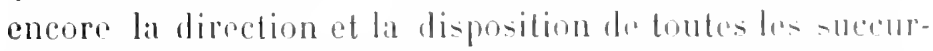

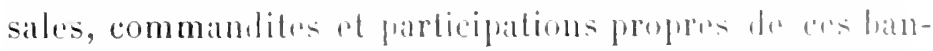

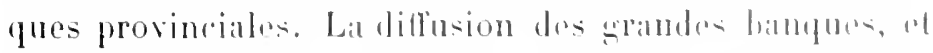
par lit lours mogens dixploitation, se tronvent andinsin-

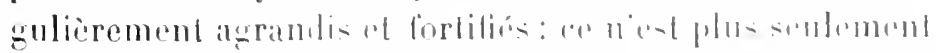

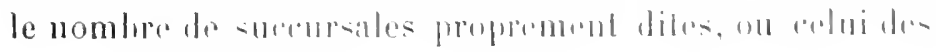


agences et des caisses de dépòts qu'il faut voir, c'est tout cet ensemble qu'il nous faut considérer: les tableaux suivants montreront la véritable physionomie de cette expansion des grandes banques:

\section{Groupe de la Deutsche Bank.}

\begin{tabular}{|c|c|c|c|c|}
\hline & $\begin{array}{l}\text { Filiales- } \\
\text { Agences }\end{array}$ & Commandites & Participalions (1) & Total \\
\hline Deutsche Bank. . . . . . & 56 & 3 & 13 & 72 \\
\hline Bergisch Märkische $\mathbf{k}$. . . & .15 & 3 & - & 13 \\
\hline Schlesischer Bankverein. . & 9 & 3 & - & 12 \\
\hline Ilannoversche B. . . . . & 6 & - & 2 & 8 \\
\hline a) Osnabrücker B . . . & - & 2 & - & 2 \\
\hline b) Hildesheimer B. . . . . & 1 & 2 & - & 3 \\
\hline Duisbura-Ruhrorter B. . . . & 2 & - & - & 2 \\
\hline Essener Credit Anstalt. . . & 10 & 2 & 1 & 13 \\
\hline a) Westäflischer Bkv . . . & 1 & - & - & 1 \\
\hline Siegener Bank. . . . . . & - & - & - & - \\
\hline S̈̈chsische Bank. . . . . . & 8 & - & - & 8 \\
\hline $\begin{array}{l}\text { Essener Bankverein. . C } \\
\text { Oldenburgische Spar u. Leih }\end{array}$ & 3 & - & - & 3 \\
\hline Bank.......... & 6 & - & - & 6 \\
\hline Emdener Bank. . . . . . & 1 & - & - & 1 \\
\hline $\begin{array}{l}\text { Prival bank zu Gotha. . } \\
\text { Mecklemb. Hypotek u. Wechs. }\end{array}$ & 3 & - & - & 3 \\
\hline Bank. . . . . . . . & 61 & - & 1 & 62 \\
\hline a) Mecklemb. Spar Bank. . & 75 & - & - & 75 \\
\hline liheinische Credit Bank . . & 13 & 3 & 1 & 17 \\
\hline (a) Süddeulsche Bank. . . & -- & - & - & - \\
\hline & 270 & 18 & 18 & 306 \\
\hline
\end{tabular}

(1) Ne sont pas comprises les participations dans les banques coloniales:

Deutsch Asiatische Bank. . . . . . 7 succursales

Deutsche Treuhandgesellschaft.

Deutsche Ueberseeische B. . . . . . 11 -

(sons le nom de Banco Aleman Transatlantico.)

Banca Commerciale Italiana. . . . . . 23 -

boutsche Ostafrikanische Bank. 
2. Ciroupe de la Discontogesellschaft.

Norddeutsche Bank. . . . . .

Allgemeine Deulsche Cred. A. 1

Rheinische Disconto $\mathrm{i}, \ldots . .11$

a) Bochumer B........ . 2

b) Eschweiler B. . . . . - -

c) Dürener B. . . . . . . -

d) Volksbank (ieilenkirchen.

e) Oberbergische R. . . .

Eupener Credit b.

Barmer liankverein.

Bayerisch. Discontogesellsch.

sïddeutsch. Discontogesellsch.

\begin{tabular}{rrrr}
2 & - & - & 2 \\
1 & 1 & - & 2 \\
\hline 62 & 7 & 15 & 84
\end{tabular}

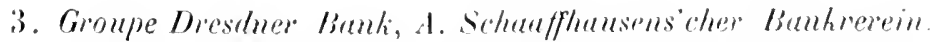

\begin{tabular}{|c|c|c|c|c|}
\hline & $\begin{array}{l}\text { Agences } \\
\text { Succursales }\end{array}$ & Conmindtles & Parlicipations & Total \\
\hline Dresdner Bank. . . . . . & . 43 & 1 & 8 & $\$ 2$ \\
\hline Schaalfh. Bkv. . . . . & 23 & 1 & 3 & 27 \\
\hline Mïkische Bank. . . . . & 8 & - & - & 8 \\
\hline Rheinische B. . . . . . & 3 & - & - & \\
\hline Oberschlesische B. . . . . & 1 & - & - & \\
\hline Wiirttembergische Landesb. & 2 & - & - & 2 \\
\hline Hecklemburgische B， . . . & 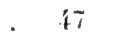 & - & 2 & 49 \\
\hline a) Ro-tocker 13..... & 11 & - & & 11 \\
\hline b) Neusorpommersche 13. & 8 & - & - & \\
\hline Landgrafl. llessische Landesb & 1 & - & - & 1 \\
\hline Schwarzburgische Landesb. & 4 & - & - & \\
\hline Ostdeutsche 13. . . . . & 2 & -- & - & \\
\hline Pfälzische B. . . . . . . & 23 & - & $\cdots$ & $2: 3$ \\
\hline Mittelrheinische $\mathrm{B}$. . . . . & 2 & - & 1 & $\dot{t}$ \\
\hline a) Mülheimer B. . . . . & 2 & - & - & 1 \\
\hline Oldenburgische landesb. . . & i!! & - & - & 89 \\
\hline & $2 \cdot 2 ! !$ & $\because 2$ & $1 \%$ & 20 \\
\hline
\end{tabular}

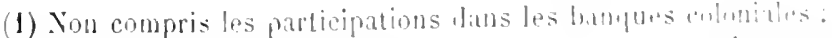

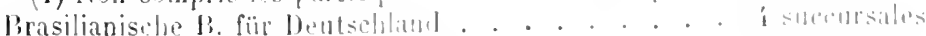
Bassa gerevale Romanal. . . . . . . . . . . i Damara und Namaena Jambelsurellechaft. . . . . : 3 Bank fur Chile und Deulsollanl . . . . . . . . 7 Cie Commerciale Breltere 
4. Groupe de la Darmstäder Banl.

\begin{tabular}{|c|c|c|c|c|c|}
\hline & & $\begin{array}{l}\text { Agences } \\
\text { Succursales }\end{array}$ & Commandites (1) & Parlicipations & Total \\
\hline Darmslïdter Bank. . & & 20 & 7 & 4 & $3 \mathfrak{t}$ \\
\hline Breslauer Discontobank & & 7 & 3 & - & 10 \\
\hline Ostbank für H.u. G. . & . & 8 & 1 & - & 9 \\
\hline a) Bromberger B. . & . & - & - & - & - \\
\hline Nordwesldeutsche B. . & . & $\breve{5}$ & - & - & 8 \\
\hline \multirow[t]{2}{*}{ Vereinsbank . . . } & & 60 & - & - & 60 \\
\hline & & 100 & 11 & 4 & 115 \\
\hline \multirow{4}{*}{$\begin{array}{l}\text { Mitteldeutsche Credit B } \\
\text { Nationalbank . - . } \\
\text { Commerz u. Disconto B }\end{array}$} & & 20 & 2 & - & 22 \\
\hline & & 14 & 1 & 1 & 16 \\
\hline & & 27 & 2 & - & 29 \\
\hline & & 63 & 5 & 1 & 67 \\
\hline
\end{tabular}

Total gènéral:

Deutsche Bank. . . . . 306 établissements et succursales.

Dresdner Bank . . . . . 24.3 》 ”

Discontogesellschaft . . 8 8; "

Darmstiidter Bank. . . 115 " "

Mitteldeutsche Credit Bank. 22 " "

National Rank. . . . 16 " "

Commerz u. Disconto liank. 29 "

817

4" Associations d'Intéréts.

(Interessengemeinschaften).

Aussi bien, trouvons-nous encore dans l'histoire de la concentration des banques allemandes de ces toutes dernières années, un dernier et plus puissant encore moyen de concentration. Il s'agit des Interessengemeinschaften, (communautés, associations d'intérèts), alliances proprement dites, sanctionnées par de véritables contrats, conclus

(1) Non compris la Wechselstuben Actien Gesellschalt "Mercur " à Vienne (i succursales). 
pour une période déterminér entre drux étiblisiemunts de foree sensiblement égale. Chacune des deux bangues va conserver sa direction et sa personmalití propeses: it n'y a ni échange d'actions, ni rémion de l'aroir des deux sociétés ainsi associées; mais les aflaires seront conduites de concert et les bénélices nets partagrís proporlionnelle. ment au capital et aux réserves des deux établissements; on a dit, et lexpression parait juste, que cétait lì un cartel de banque.

Nous eroyons pouvoir donner comme précédents immé. diats et comme préparation de ces communautés d'intérits, ce qu'on appelle les consorliums de banques, éestit-dire ces accords momentanós. sociótós oceasionnelles Golegenheitsgesellschaften) (1), gronpemonte temporaires de banques, conclus pour la conduite d'une affaire déterminée importante, une émission d'emprunt publie par exemple. La direction et la comptabilití sont alors réunies entre les mains d'une seule maison: ha toute puissance des banques d'émissions résile dans eete organisation unitaire et dans la centralisation du capital entre les mains de l'établissement directeur.

A vrai dire, les consortiums ne sont point particulirs aux banques allemandes: nos gramdes socifités franciatioes ont pris également l'habitude de se grouper pour la conduite des grosses alfaires: mais on . Iltemalgne, fes ronsortioms sont une habitude couranto (2) et la multiplicilé

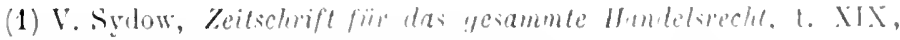
p. 427 ; E. Goldschmilt ; The Binlitgruppen.

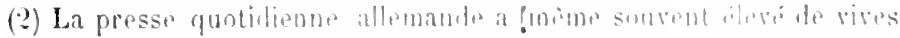

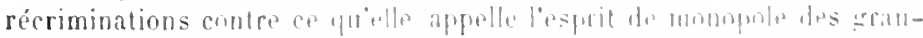
des banques; il siurit surtout daus ces furiutes, hes empronts commu- 
des affaires consortiales, inscrites au Konsortial Konto de chaque établissement (1) leur donne un caractère permanent en quelque sorte, virtuellement du moins; mais la communauté d'action est toujours bornée à la conduite des affaires désignées.

Nous donnons ici la liste et la composition des grands consortiums.

\section{Consortium de Prusse.}

Reichsbank.

l'reussische Seehandlung.

rreussische Centralgenossenschafstkasse.

Bank für Handel und Industrie. Berliner Bank.

Berliner Hundelsgesellschaft.

s.' Bleichröder.

Commerz und Disconto Bank.

Delbrück Leo et Cie.

Deutsche Bank.
Deutsche Genossensehaftsbank. Discontogesellschaft.

Dresdner Bank.

F. W. Krause et Cie.

Mendelssohn et Cie.

Mitteldeutsche Credit Bank.

National Bank.

Schauffhausens'cher Bankverein.

Schickler frères.

R. Varschauer et Cie.

\section{Groupe Rolhschild.}

Discontogesellsehaft.

()sterreichische Ciredit Anstalt

fiir llandel und liewerbe.

II. Wodianer.

S. M. von Rothschild (Viemne).

Ungarische Allgemeine Credit Bank (Pest).

s. Bleichröder.
N. M. Rothschild et fils (Londres).

Allgemeine Osterreichische Boden Credit Bank.

Bank für IIandel und Industrie. Sal. Oppenheim (Cologne).

Mendelssohn et Cie.

Osterr. Postsparkasse.

Rothschild frères (Jaris).

naux conduits par les consortiums qui contiendraient un grave danger pour les villes en les livrant sans défense aux mains des syndicats fuanciers. Mais il semble bien difficile, étant donné le volume des emprunts publics nodernes, dont l'importance dépasse aisément la capacité d'une banque particulière, d'éviter la formation de ces consortiums.

(1) V. daus la 2 partie l'étude du Konsortial Konto de chacun des grands établissements berlinois. 
Consorlium des emprunts linsses.

Mendelssoln et lie.

S. Bleichröder.

Discontoyesellschaft.

R. Varschater et lie.

Volgakama Commerzbank. letersburger Internat-llandelsbauk.

Riüssische bank lïr auswirligen llandel.

Petershurger l)iscontobank.

Lippmann, Rosenthal et lie (Amsterdam).

Syndicat des affaires asiatipues.

Dentsch-.tsiatische liank.

Discontuyesellschaft.

Deutsche Bank.

S. Bleichröder.

Berlines Handelsyesellschaft.

Bank fïr Handel u. Industrie.

Jacob II. S. Stern.

Norddeutsche Pank.

Sal. Oppenheim.
Preussische Seehandlung.

Bayerische Hypotheken und

IVechisel bank.

Dresdner Banli.

schanffhamens'cher bilie.

born el liusse.

Vitional Bami.

L. Hehrens et fils.

Dans chacun de ees consortiums nous voyons réunis les grands établissements berlinoi: : nul doute que l'habitullo ainsi prise de mener le concert un groupe d'alfiares n'ait conduit a ces alliances plus átroites - permanentos et non plus monentanése, ambrassint lonsomble des opórations et non plus senlement quelques affinies díterminées - que nous voyons réalision par les communantés d'intérèts contractuelles.

Ces communautís d'intírits sont enere latros: nous en arons relevé quatre:

Les Interessengemeinsehalton de la Demesche lianti aree

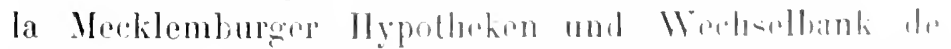

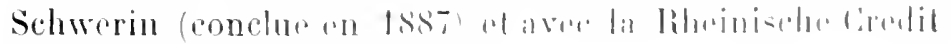
Bank, conclue en lan't.

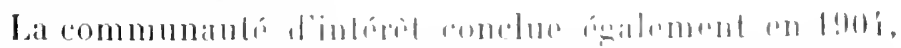

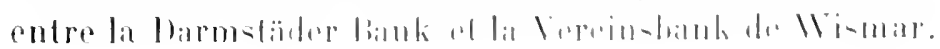


La communauté d'intérèts Dresdner Bank-Schaaffhausens'cher Bankevein : c'est la plus importante et la plus complète ; elle réunit des capitaux formidables : 349 millions de marks. Nous allons l'étudicr avec quelques détails.

Nous avons vu déjà, en examinant au point de vue de la concentration la crise 1900-1901, l'élan que vint donner au mouvement d'expansion des grandes banques, l'exemple - et les rapides progrès - de la Deutsche Bank, et comment l'accord intervenu entre la Dresdner Bank et I'A. Schaaflhausens'cher Bkv. (décembre 1903) en avait été une des principales conséquences. D'autres circonstances vinrent faciliter cet accord. La Dresdner Bank avait besoin de développer ses affaires de banque pure; or, celles de l'A. Schaafhausens'eher lui donnaient depuis longtemps des résultats excellents. D'autre part,cette dernière ayant besoin d'améliorer et de dévolopper ses participations industrielles était sùre de trouver un appui certain auprès de la Dresdner Bank.

Enfin, et c'est là sans doute l'essentiel, les établissements propres des deux Sociétés, leurs filiales et leurs participations étaient situés géographiquement de façon telle, qu ils se complétaient à merveille et allaient donner à la communauté d'intérêts une puissance égale à celle qu'aurait procuréc une fusion.

Ces motifs et les bases de l'entente ont été ainsi exposés dans l'assemblée générale des actionnaires de la Dresdner Bank (1903):

"Depuis longtemps notre attention était dirigée vers l'extension de notre sphère d'intérèts dans le grand district industriel du Rhin et de Westphalie, dont l'importance pour l'ensemble de la vie économique allemande va 
encore grandir far les proupements existants et fug qunpements en formation. Xous avions axaminr phasieurs combinaisons dins cet orlde d'idées sans cependant nous lier à une forme détermináe. Récrmment l'occasion sest offerte de nous rapprocher la plus ancien ot du plus puissant établissement rhénan, le Schathliausens'cher Bankverein qui cultive depuis plus d'un demi-sicele les relations les plus préeicuses avec l’industrie: nous arons saisi avee empressement cette oecasion de réaliser d'un coup notre projet. Nous eroyons, en effet, qu'i preine il existe deux institutions qui se completent aussi heurensement, le Schadfhatusens'cher bankverein avec son excellente clientèle de l'Allemagne Gecidentale el la Dresdner bank qui en dehol's de ses relations industrielles an Saxe et dans d'autres districts entretient un résanu de succursales à travers toute l'Allemagne, el de Hambourg, Brène et Londres, cultive avec un suceès grandissant, les alfaires transatlantiques."

"La forme choisis de communaute d'imtripts se distin. gue des fusions at antres combinations analognes en ce qu'elle évite de diminuer le divilende des actionnaires par des frais inutiles de timbre, par une double taxation, par de doubles tantiemes. Les propositions summises a nos aclionnaires ont été adoptées à l'unamimité, parles direce. teurs et les membres du Conseil.

"L'entente a été conclue, it dator du lor janvier lon't, pour trente ans. Chacune des deur sucirbés conserve ealle-

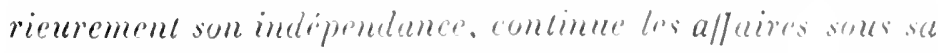

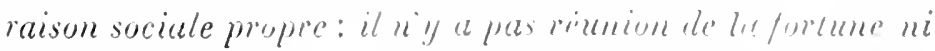
de la personnalité suciale, mais les affaires sond combites en commun, les bentices somb rímis et distribués an prorala 
du capital social et de la réserve de chaque société. Ainsi nous eroyons obtenir d'une façon approximative les mèmes avantages économiques que par une fusion et éviter d'autre part les nombreux inconvénients, les dépenses improductives de timbre (I), la double taxation par l'Etat et par les communes.

"La réunion du bénéfice annuel aura lieu de la manière suivante: chaque Société établira un compte de profits et pertes sans tenir compte de la communauté, comme si celle-ci n'existait pas. Du bénéfice brut, après soustraction de tous les frais généraux, de la dotation des réserves, il sera déduit les tantièmes des administrateurs, directeurs, employés, les gratifications, les amortissements d'immeubles. Le solde qui reste (bénéfice net) va dans la mème masse et il est partagé d'après le mode indiqué ".

"Chaque sociélé établit, après que sa part de bénéfice net aura été calculée, son bilan définitil et son compte de prolits et pertes, sans être dépendante pour l'emploi de sa part de bénélices nets, des organes de l'autre Société. La part, qu'après l'établissement du décompte une Société devra payer ì l'autre, figurera comme perte ou profit dans le compte des profits et pertes, diminue le bénéfice de celui qui paie, augmente le gain de celui qui reçoit. Le calcul des tantièmes du Conseil el de la Dircetion doit être établi sur le résultat définitif. Chaque Société a le droit d'employer à sa gruise le bénéfice net, c'est-ì-dire de créer éventuellement des réserves. La communauté d'intérêts portesur les bénéfices annuels, non sur les pertes. Si une Société a subi une perte clle doit couvrir cette perte, en tant que le bénéfice provenint de l'autre sociétés ne la

(1) On a calcule que les frais d'enregistrement seuls auratent atteint 5 millions de marks. 
compense pas, en prenant sur son fomb de réserve an alltrement, de façon que te bénéfice anmuel do l'annón suivante ne soit pas diminue par un rósidu de perte. Eu r.e qui concerne le coeflicient de la répartition, en dehor-du fonds de réserve légale, il n'y a à truir comple que des fonds de réserve figurant dune façon permanentean bilan el qui ne constituent pas seulement des réserves contre des pertes spéciales."

"Afin d'assurer une commmnate de direction, il rst prévu que chaque société fouruira doux directours dol trois administrateurs yui seront complés aux orwanes de l’autre: le Conseil dauministration de chaque sociétí, pour la préparation desaffaires importantes. éliradalus son sein un comiti auguel prendront part doux membreste l'autre société, en règlo générale. les directents : los comilés réunis formeront un Consuilde délegation. Celui-ei devra décider en cas de divergence d'opinions, en ex qui concerne lapprobation des bilans provisoires. des comples de prolits et pertes, la répartition entre les deux Suciólós, les amortissements; il aura une influnce particulicre en ce qui touche les augmentations de capital : me ausmontation de capital hite sans son approbation ne sera pats prise en compte lors de la répartilion du bínéfice al donns droit a l'autre socirté de se rolirele le la commumantí. 'Toute modilication dans les arrangements derra itre approuvée par les deux tiers des administrateurs. "

En vue de cotterntente, chacun desdenx établicsement-

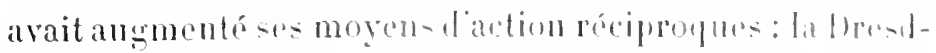
ner Bank portint som capital d, l:30 it leil) millioms de

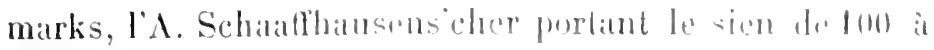
$12: 3$ millions de matks. 
L'activité du nouveau groupe se manifeste tout d'abord par une série d'absorptions; il aequiert un siège à Francfort par l'absorption (Dresdner Bank) de la vieille maison Erlanger et fils fondée en 1859 et intéressée elle-même dans les entreprises suivantes:

$1^{\circ}$ Oldenburgische Landesbank.

$2^{\circ}$ Mecklemburgische Bank et ses deux participations:

a) Rostocker Bank.

b) Neuvorpommersche Spar. Creditbank

$3^{\circ}$ Landgräflich Hessische Landesbank.

$4^{\circ}$ Eisenbahn Rentenbank.

כ0 Eisenbahn Bank.

$6^{\circ}$ Frankfurter Hypothekenverein.

$7^{\circ}$ Schwarzburger Hypothekenbank.

La Dresdner Bank absorbe encore la succursale de la Deutschegenossenschaftsbank à Francfort, lui cédant 20 millions de ses nouvelies actions et lui payant en espèces 3.750 .000 marks.

De son côté l'A. Schaaffhausens'cher Bankverein absorbe :

10 la Westdeutsche Bank;

$2 \circ$ la Niederrheinische Gredit Anstalt;

Au début de 190כ̃ enfin, la " communauté d'intérêts" absorbait la Treuhand Vereinigung Actiengesellschaft de Berlin.

On voit, en dehors du résultat brut de la réunion des capitaux et réserves des deux établissements, l'essor donné au mouvement de concentration par la conclusion de cette communauté d'intérèts. Il serait intéressant d'en suivre le fonctionnement et l'évolution. Elle est encore trop rapprochée de nous pour que nous puissions en juger les résultats avec certitude; citons cependant ces ex- 
traits des rapports gínéraux (190') des deux ítablisements :

"Notre activilé pour celte annéx, dit le rapport dr la Drestner Bank, était consacréce en premierr ligne a liarganisation de notre "Interessengemeinschalt nalrec l' I. Schaalfhasens eher Bankverein. Les mesures prises se sont monlrées excellentes: lientente a été parfate et lat marchedes alfaires na point íté rendue phos difficile par le fonctionnement dun appareil plus compliqué. "

- "Lannéequi vient de sécouler,dit de son còté le rapport de l'A. Sehatafhausens'cher bankrerein, a vu sachever lorganisation de notre communatós d’intriets avec la Drestner Bank. Las résultats obtenus témoignent de la puissance te lidéc qui presida à eet accord: il en est résulté pour les drux banques mne angmentalion d influenec. un élargissement de leur activité nous espórons voir augmenter encore par la suite le nombre des atliares. "

Tels sont les princifaux moyens quemployerent les grands Instituts berlinois pour rúaliser la concentration. Nous arons ru rn quoi ils ditherent protondément des méthodes généralement emplopées en Angletere al en France; ils nous apparaissent plus nuancés, plus souples, plus économiques aussi, tout en assurant une larga diflusion et de fait. devant les pésultat- aujourd'hui acpuis. on

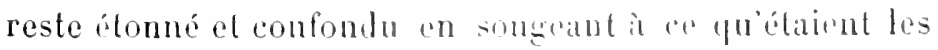
banques allemandes il y a seulement ringt ans.

11 nous reste à montrop comment les gramblis ítabliste-

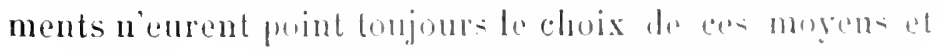

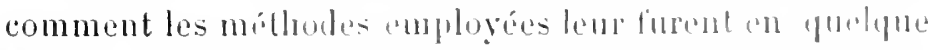
sorte imposés par le développentent historigue de l’. Illemagne elle-mente. 
Nous avons vu comment ces banques qui plus tard, vers 1890, sont devenues les grands élablissements berlinois avaient commencé pour la plupart, par être de simples banques provinciales; elles prirent la tête da mouvement industricl et commercial et, devenues plus puissantes, vinrent s'élablir à Berlin.

Mais les autres banques provinciales fondées de $18 \% 3$ à $187 \%$, pour n'avoir point suivi ees quelques banques, plus favorisées ou mieux conduites, dans leur magnifique essor, n'en avaient pas moins travaillé et prospéré ; établies dans les centres manufacturiers importants, dans le bassin Rhénan par exemple, ou dans les villes commerçantes du Nord et du Centre de l'Allemagne, elles avaient, elles aussi, grìceà la décentralisation économique fortifiée par les vestiges d'un particularisme provincial encore très vivace, lurgement contribué pour leur part à l'essor du commerce cl de l'industrie et fondé, dans leurs environs immédiats, un réseau d'agenees qu'alimentait une solide clientèle.

A vrai dire, ce ne sont point des banques locales mais lien plutòt des banques régionales que trouvèrent devant eux les grands átablissements berlinois que la concentration eroissante du tralic de banque à Berlin obligeait à s'étendre, à rayonner à travers les provinces. L'activilé industrielle et commerciale de nombreuses régions permit à ces banques régionales de conserver un champ d'opérations assez fructueux et elles résistèrent an début avec un certain suceès aux tentatives d'expansion des grandes banques. Le premier essai de la biseontogesellschaft de fondre un certain nombre de banques provinciales en une Société par actions, la Provinsial Discontoyesellschaft, échoua presqu'aussitôl: la Provinzial Wechslerbank, création de la Berliner Wechslerbank n'eut pas plus de succès. 
Si, vis-it-vis des banques priveres, les banques forlinuises - puissamment secondées d’aillenrs par l'action l, ans eauses particulières que nous avous examinres et qui, dr

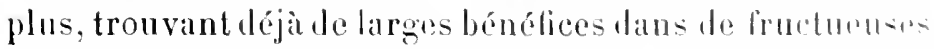
opérations financières lirent, pour gagner la clicntible, de notables concessions dans le tratie rigulier de banqunpurent agir par voie d'absorptions et de fusions complielis, elles durent user à l'égand des banques provinciales du moyens plus souples et de méthodes moins brulales.

Aussi bien comprirent-ollesque si elles devaionts'ítemdre pour assurer l'aceroissement ide leurs aflaires combutes de banque, nécessité par le développement des imalustries anxquelles elles sontéressident. comme coalement pour fortilier leur action sur les lieux mimes vì are industries sexerçaient, point nétait besoin pour cola de fusionner completement avec les hanques provinciales; plutòt peut-être que les avantages que devait présenter une politique d'unions et d'alliances, elles surent roir les inconvénients d'une centralisation trop compliete et trop absolue. Ce quilles cherchorent arant tout, le palsiage du rapport de la Dresdner Bank yue nous arous rite le dit explicitement, c'est un compliment de lours moyens d'action et ce complément elles l'obtimpent plus sìnenunt en laissant ì leurs collaborateurs, it leurs allies, ol phos d’indépendanee et plus d’iniliative.

Le mourement a done abouti à celle concentracion de forces que nous arons analysee, non à la própromelírame

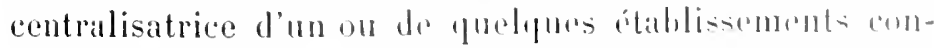

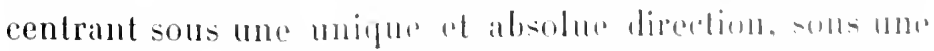

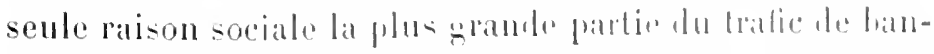

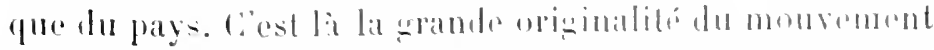
de concentration dans les liampues atlembandes. Lal jubli- 
cieuse observation de M. Blondel: "les Allemands semblent aujourd'hui convaincus que c'est dans une sorte d'équilibre entre la concurrence individualiste et l'association qu'il faut chercher le véritable régime économique du monde moderne " (1), trouve ici une entière application.

(1) Blondel, op. cit., p. 280 . 


\section{DELXIEAL PARTIE \\ ÉTUDE ANALYTIQUE DU MOUVEMENT DE CONCENTRATION}





\section{DELXIEME PARTIE}

\section{ÉTUDE ANALYTIQUE DU MOUVEMENT DE CONCENTRATION}

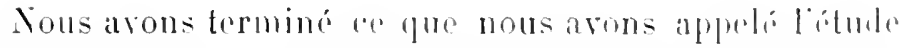
sỵnthétique du mourement de concentration dans les lainques allemandes. On sait maintenant quelles en furent les causes générales et particulieres; on connait les móblundes employées el l’importance des rúsultats obtenus an as quinze dernières anmés. Mais ee niest lit, arone-mous dit, quiun des aspects de l'ítude du mourement de concentration.

Il nous reste it lenvisager it un point de vur imterne. cest-ì-dire à déterniner la part spéciale que vint premder

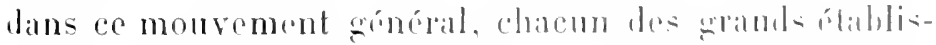
soments chefs de groupe, sun rite pathiulier, ses tendances propres, le résultal particulior yu’il olulint.

Les grands institut berlinotis nout point tous atppertí.

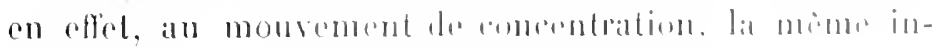

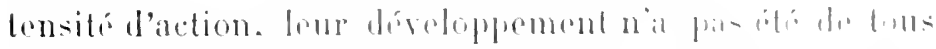

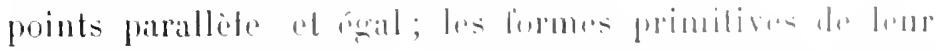

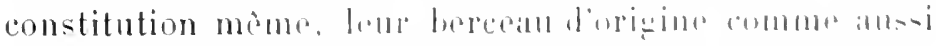

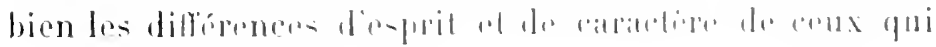


furent placés à leur tète, ont développé des influences qui déterminèrent le sens de leur activité et le choix des moyens qưils utilisèrent pour s'agrandir et s'étendre. Nousétudierons done pour achever de donner une physionomie exacte du mouvement de concentration, chacun des cinq grands établissements conducteurs, Discontogesellschaft, Bank für Handel und Industrie, Deutsche Bank, Dresdner Bank, A. Schaaffhausens'cher Bankverein, nous plaçant pour chacun successivement au triple point de vue suivant:

$1^{\circ}$ De son développement propre. - L'examen des bilans généraux que nous avons résumés jusqu'en 1894 et donnés plus en détail pour ces dix dernières années montrera les progrès de chaque banque comme aussi ses périodes d'arrêl, vite dépassées d'ailleurs; une activité plus grande de la banque a jusqu'ici toujours suivi les reculs provoqués par les crises économiques (1873-1891-1900-1901). La rapidité avec laquelle les grandes banques allemandes ont, en particulier, réparé les effets de la dernière crise, diminue singulièrement la portée des accusations ou seulement des jugements trop sévères qui ont été parfois portés sur elles (1).

Nous avons aussi donné d'une façon plus analytique l'origine des bénéfices, bénéfices provenant des affaires courantes de banque et bénéfices provenant des titres et participations. Ce qui a été dit au chapitre II (Cause générale technique) montre l'intérêt de cette distinction comme

(1) Sayous, Banques de depôt et de crédit... p. 292, 293, "une panique grave, même pas trés grave forcerait la presque totalité des banques allemandes à suspendre leurs paiements ", etc... Voir également "Les banques allemandes en cas de crise ou de guerre " Revue d'économie politique, février 1899 . 


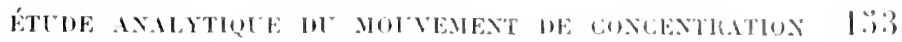

aussi bien olle sert ì caractériser l'activité parliculiore d. charfue banque.

Les indications qui aceompagnent ees bilans sont formiment courtes et per nombreuses; nous nous sommes platcé uniquement au point de vue de la concentration. La rémion de ces documents qui n’a jamais encore itri faitu en France et quen Mllemagne. on trouve soit ícourtés ut disséminés dans des onvrages de dilférentes éporpes. soit lrès condensés et prósentés sous lesdeux seulos rubripure: Ensemble des banques allemandes. - Banques beplinoises, - pourra faciliter d'autres itudes partirulieres sur les banques allemandes.

$2^{\circ}$ De la convitution et du dreploppement du youner quil contrifle. - Nous avons réuni pour clatpue liangue, lus finits de concentration que nous avons pu relever. en les exposant suivant leur ordre chrouologique et non plus sulpment suivant leur ordre logique comme dans la premiere partie de cette étude. La méthode synoptinue adoptér, permettra de bien saisir le déreloppement de chaque groupe ainsi que les procidés amplogés de prélérenes par telle ou telle banque et reproduits par les banques régionales affiliées; le processus de concentration appatraîtra ainsi à ses diflérents degrés.

Nous narons pu toujours donner le chillprexat des participations prisespar les grandiciablisioments diun- les banques secondaires. les rapportínéméraux no contentult

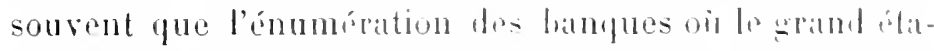
blissement est intípessi salns mentionner lo chillpo due

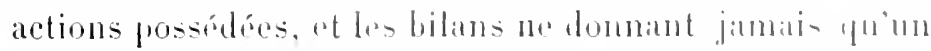

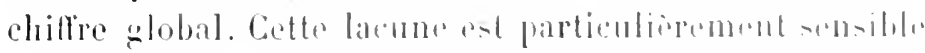

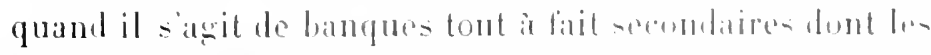

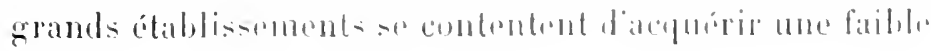


partie des actions (à moins qu'ils n'en possèdent au contraire la totalité) et c'est surtout dans ce cas un appui moral, que, croyons-nous, ils apportent.

Ces tableaux devront ètre complétés par ceux des pages 4l-45 qui donnent la lotalisation des capitaux concentrés dans chaque groupe de banques.

$3^{\circ}$ De son compte consortial et des participations prises dans les entreprises industriclles et commerciales. - A côté de la concentration des valeurs de banque en banque nous avons donné les principaux résultats de la concentration des valeurs inclustrielles en banque, conséquence de l'union étroite où se trouvent en Allemagne la banque, l'industrie et le commerce; la liste des sociétés industrielles et commerciales dans le conseil d'administration desquelles les grandes banques délèguent un de leurs membres complátera ce que les tableaux des comple consortial el compte des titres possédés en propre présentent forcément d'incomplet: les grands établissements - comme les Sociétés industrielles - se souciant assez peu de donner la nomenclature détaillée et le montant de leurs participations. - Différentes listes des principales participations prises dans les récentes émissions (fonds d'Etat, emprunts communaux, émissions d'actions de banque ou de Sociétés industrielles) montreront enfin l'activité des grands établissements berlinois, sur ce terrain particulier.

Cette seconde partie présente, on le voit, un intérèt surtout documentaire. Puisse cet intérèt être assez grand pour faire pardonner l'aridité des chapitres qui vont suivre. 


\section{CHAPITRE PREMIER}

\section{LA DISCONTOGESELLSCHAFT}

\section{I. - Le developpement propre de la Disoontogesellsehaft.}

La Discontogesellschaft fut fondée en 18.31 ì berlin prar le grand financier Davial Hansemann, ancien ministre des finances de Prusse; il aurait roulu créer ('n Allemagne, a l'exemple du Crérlit mutuel de Bruxelles 18ss. une Société du crédit mutuel à capital rariable, limitant le risque au montant souscrit par chaque membre qui verserait $100 / 0$ et auquel il serait ourert un crédit d'escompte pour le montant de ce capital souscrit.

Mais le gouvernement prussien opposa mille difficultés, et la Société dut se constituer sous forme de Société en commandite arec gérant responsable el des associcio i mise variable. Le but de la sociéli e-t nettement dilimiti: escomptes, avances, dépits produisant un intipit ant.1!-21 des statuts): tontes transictions antres sont interdites

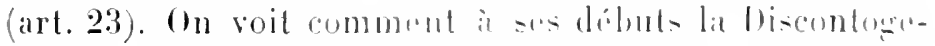

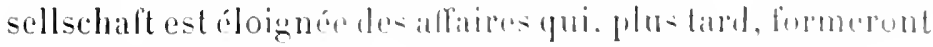

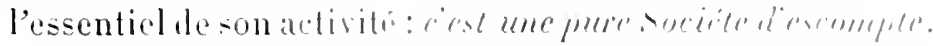

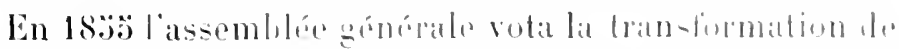

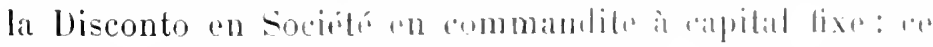

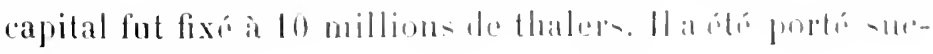
cessivement ì : 
40 millions de marks en . . . . . . . . 1871

$60 "$ " $" . . . . . .1872$

750 "

115 "

$130 \quad " \quad$ " $\quad \ldots . . . . . .1898$

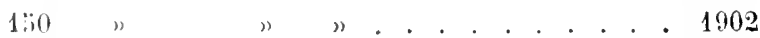

$170 "$ " $" . . . . . .1904$

Ces élévations du capital social ont coïncidé avec un accroissement général et régulier de l'activité de la banque. Si l'on excepte l'élévation de 18900 nécessitée par Yabsorption de la Norddeutsche Banli de Hambourg, celle de 1904 nécessitée par la création d'importantes filiales, les autres augmentations nont point été la conséquence d'absorptions on de participations prises dans les banques secondaires. La forme juridique particulière de la Discontogesellschaft, non moins que sa situation prépondérante dans le consortium Rothschild lui ont toujours imposé de ce còté, de grandes réserves. Ce n’est qu'en ces toutes dernières années qu'un nouvel et important développement de ses affaires de banque pure est venu nécessiter une diffusion plus grande de son activité et un élargissement de son rayon d'action (voir $\$ 1$ )

L'examen des bilans de la Discontogesellschaft nous permet de constater les principaux faits suivants :

$1^{\circ}$ L'activité de la banque a toujours été en progressant d'une façon régulière; prudemment gérée, elle ne paraît pas avoir trop souffert de la grande crise de $18 \% 7$ et celle de 1900-1904 l'a touchée beaucoup moins que certaines autres de ses grandes concurrentes; mais on remarquera en sens contraire, l'arrèt des années 1870̈-1876 et 1891-1892.

$2^{\circ}$ La progression des bénélices est moins régulière et non toujours proportionnelle au volume des affaires, sauf en ces dernières années; c’est l'année 1872 qui nous 
donne le bencilice brut maximum ol,tenu, soit $2.3,8$ millions de marks: le hénéfice brut de l'année 190't n’atio que de 23,7 millions de marks.

$3{ }^{\circ}$ La distinction des bénéfices en bénéfiees provenant des alfares de banque pure et en bénéfices provenant des titres et des affaires en participation montre que la banque a inégalement développí ces deux branches du son activité.

a) Jusque vers 1866 , les atfines réguliores de banque tiennent la première place dans cettr activité; le portefeuille conmercial s'est aceru d'une firon régulière; Ic chapitre des valeurs propres restr a peu près stationnaire. Mais a partir de cette date, le portefenille commercial et les dépòts montrent une augmentation proportionnellement moins forte que celle des valeurs propres et des affaires en participation et aussi moins réguliere.

La Dicontogesellschaft est devenue arant tout une Socirté financière, intéresséte à presque toutes les grandes aflaires internationales; elle a négocié des emprunts pour le Chili, l'Autriche, la Russie, la Roumanie, sans parler des opérations faites avee l'Empire. les Etats particuliers et les villes allemandes; elle a participé il la fundation des grandes entreprises industrielles et commerciales el si de ce còté, dans la période $1866-1890$ par exemple, elle al trouvé des bénéfices considérables le plus somrent égatux ou supérieurs (1878,1879.1880, $1881,1883,188$ ) aur bénifices provenant du reguliere Geveliajl, clle a partois aussi éprouvé des pertes sérienses, témoins les énormen cipi-

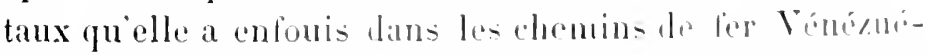

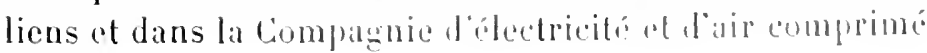
Popp.

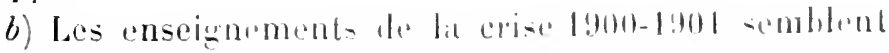
avoir eu une grande intluence sur la biscuntogesellochaltt. 
Elle a de nouveau orienté son activité vers les affaires régulières de banque; on constate en effet un accroissement remarquable des chapitres suivants : portefeuille commercial $(80,2$ millions do marks en $1899,141,3$ millions en 1904), reports et lombards (40,6 millions en $\mathbf{1 8 9 9 ,}$ 73 millions en 1903), dépôts (";2,1 millions en 1899, 100,4. millions en 1901), etc. Aussi bien ce sont les bénéfices provenant de ces affaires régulières qui lui ont pernis de relever son dividende dès 1902 ( 8 1/2). 11 faut noter cependant que la branche des titres et participations a donné en 1904, en bénéfices, la somme non encore atteinte de $8,600.000$ marks.

La Discontogesellschaft nous semble done allier, pour l'heure présente, dans une juste et heureuse mesure, " ees deux ordres de travaux que le président d'un grand établissement de crédit français déclarait naguère inconciliables: la conservation de disponibilités suffisantes et l'im. pulsion donnée aux entreprises industrielles ». Si son développement propre fut, à ses débuts, moins rapide que celui de cerlaines concurrentes plus jeunes, cela tient sans doute aux conditions économiques générales de l'Allemagne et dans une certaine mesure aussi à la forme même de la Société; la responsabilité solidaire des gérants réclamant peut-ìtre plus de prudence dans la conduite des affaires; mais elle a su toujours constituer de grandes réserves, son prestige est considérable; ses dernières élévations de capital, l'intensité de son tout récent mouvement d'expansion prouvent assez que la Discontogesellschaft, la plus vieille arec le Schaaffhausens cher Bankverein, des grandes banques allemandes, ne s'est pas recueiltie dans une majesté un peu sommolente et qu'elle entend participer d'une façon très active au mouvement de concentration. 
Discontogesellschaft.

15.7.it-1s!is

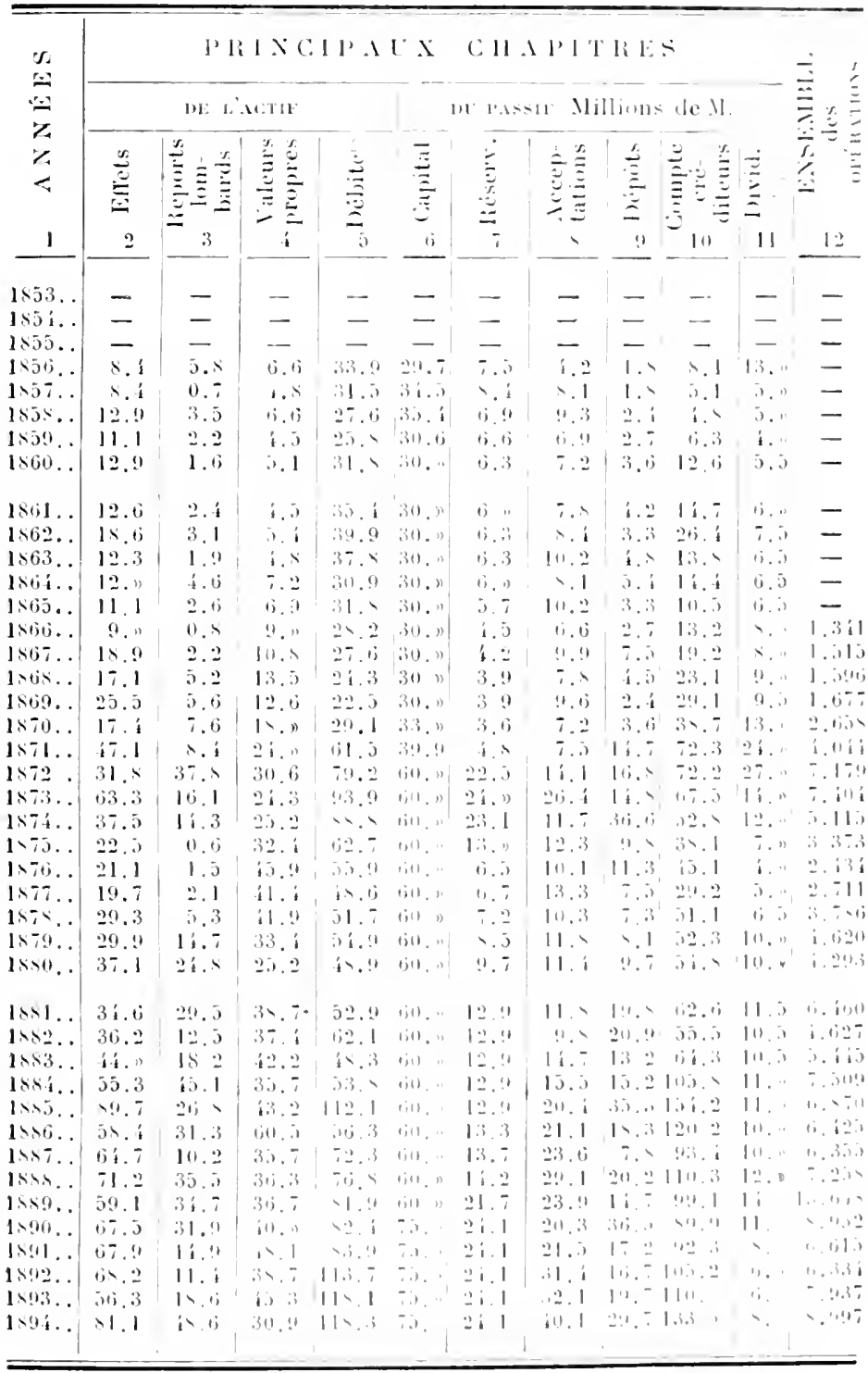




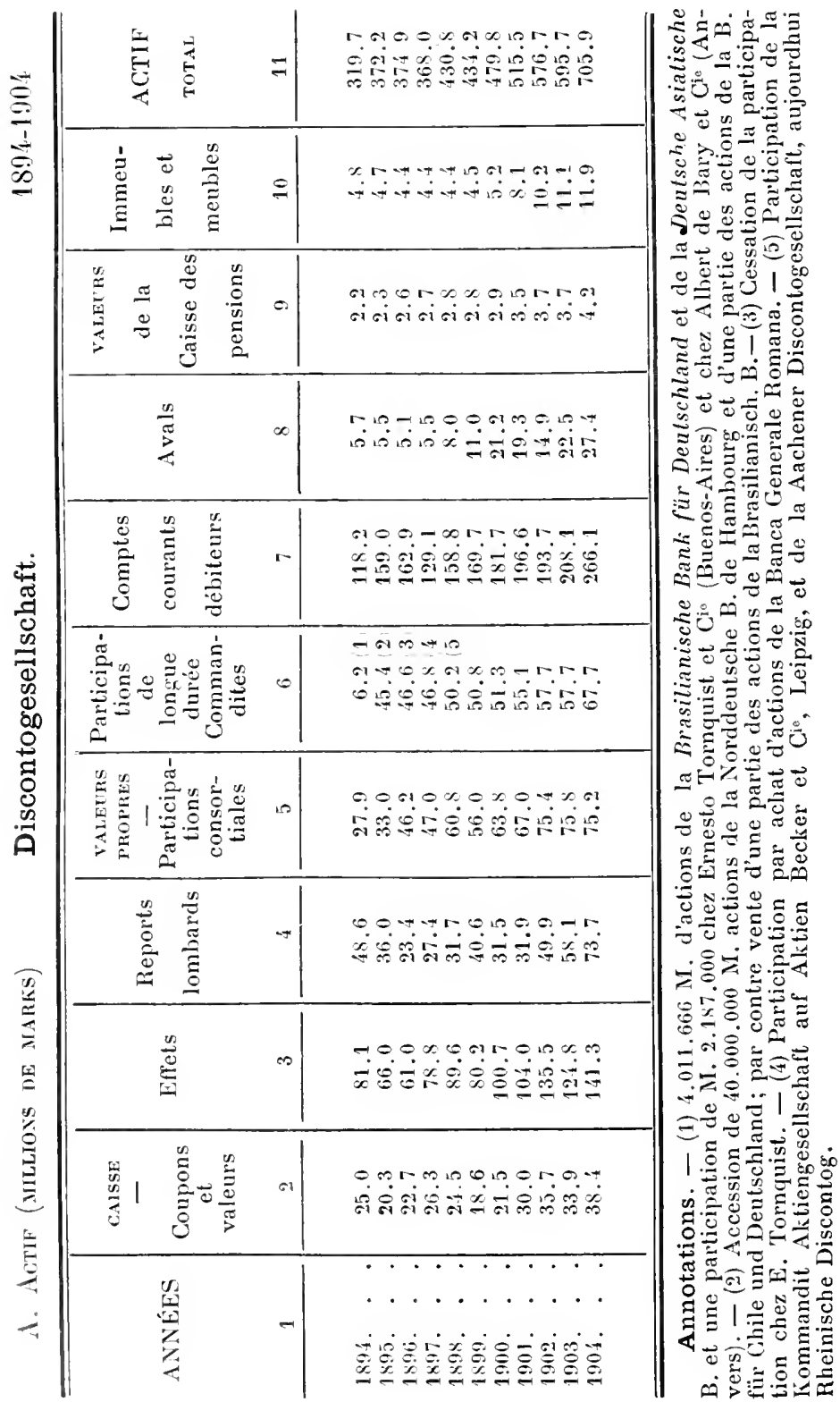




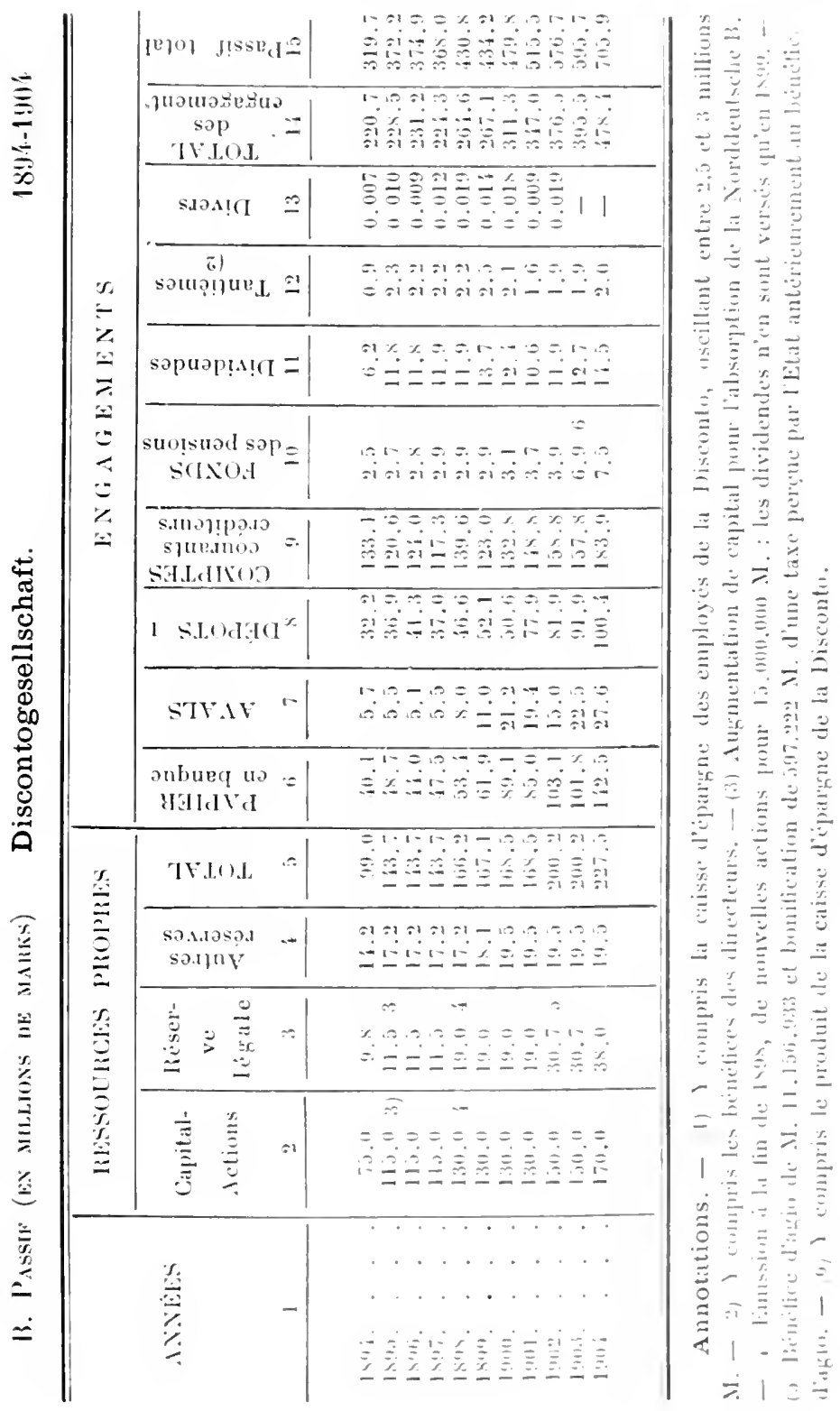




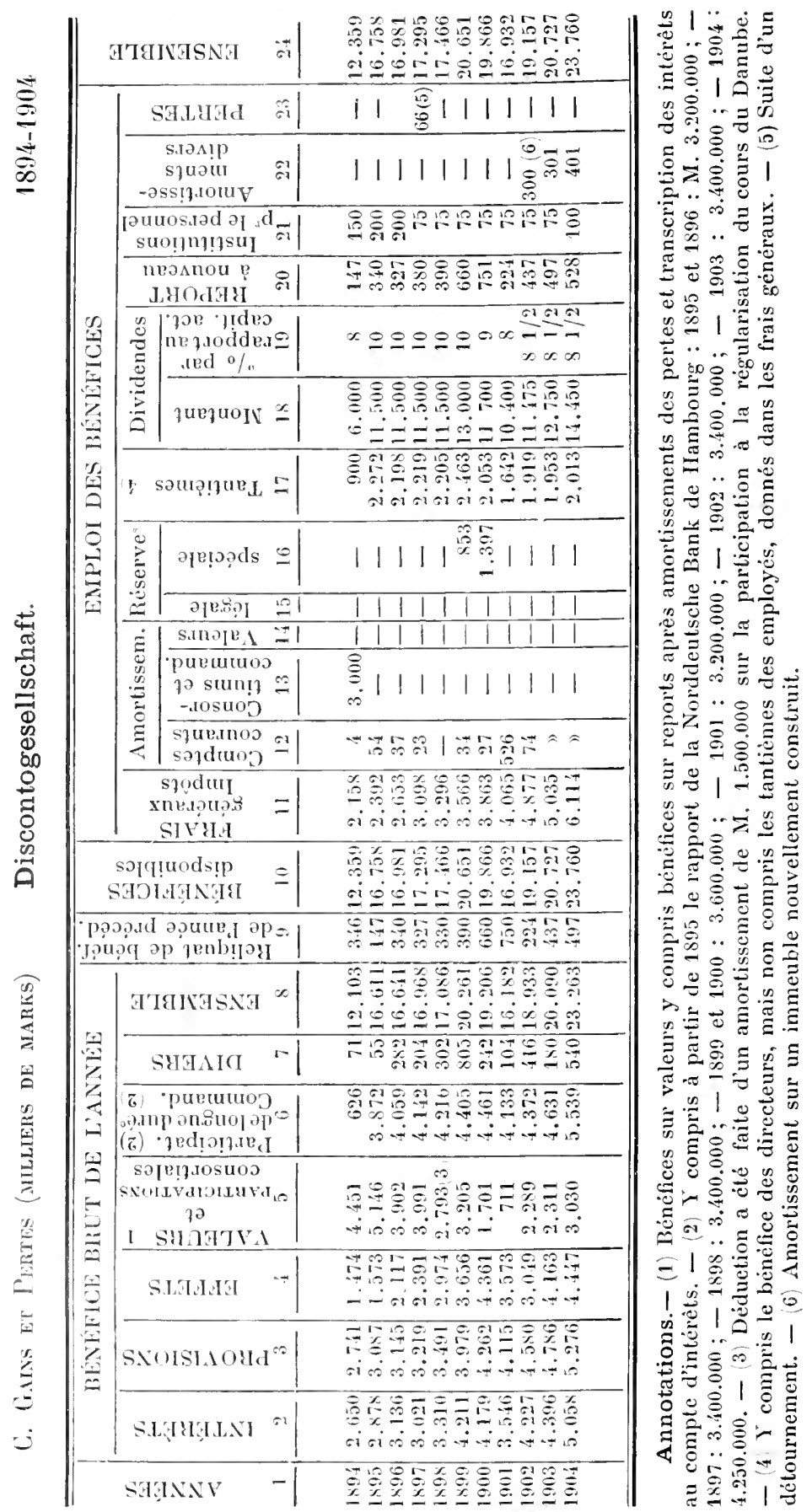


Discontoyesellschaft.

Bénéfices (millions de markis).

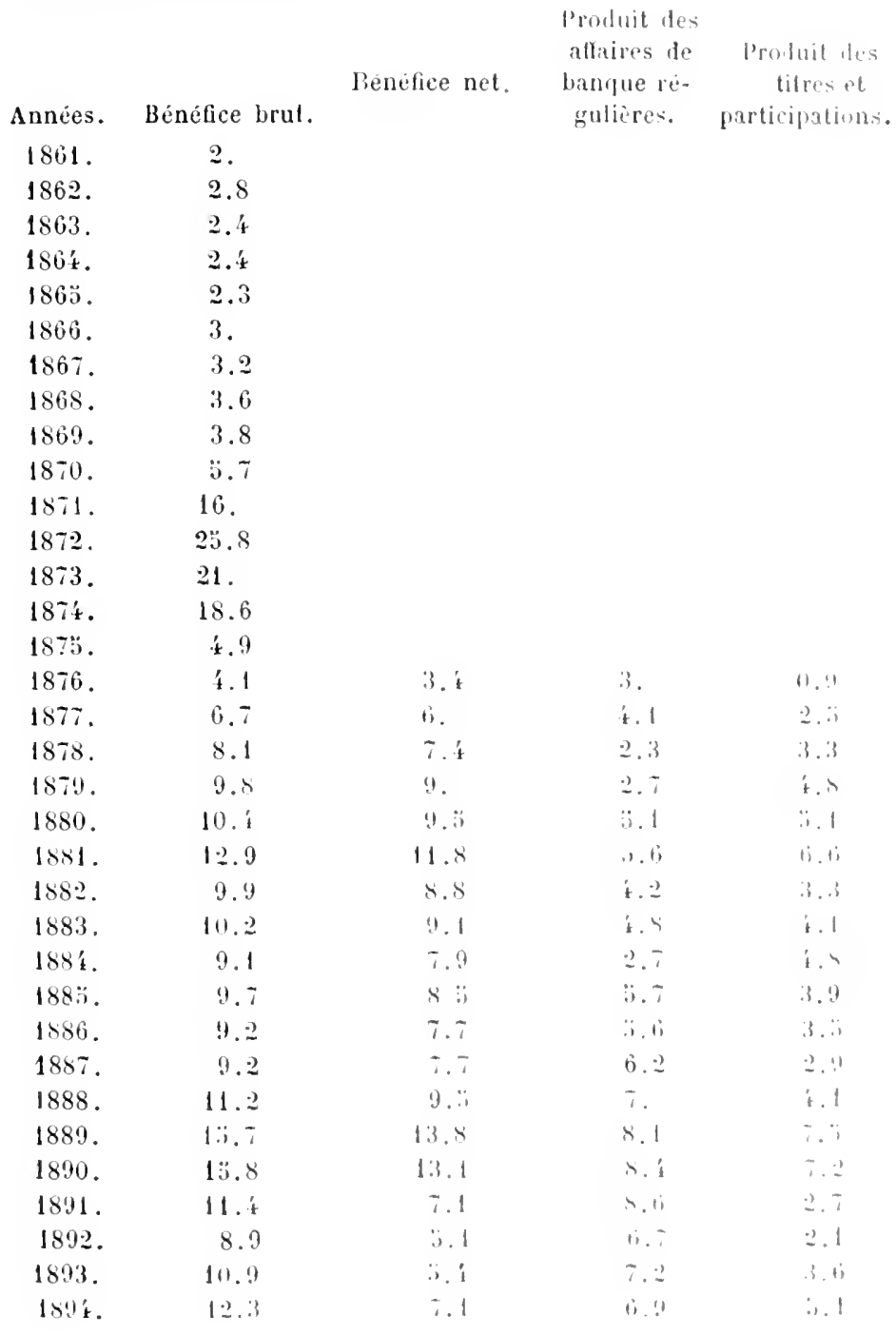


Discontogesellschaft (Suite).

Bénéfices (millions de marks).

$\begin{array}{ccccc}\text { Années } & \text { Bénéfice brut } & \text { Bénéfice net } & \begin{array}{c}\text { affaires de } \\ \text { banque ré- } \\ \text { gulières }\end{array} & \begin{array}{c}\text { Produit des } \\ \text { titres et } \\ \text { participations }\end{array} \\ 1895 . & 16.7 & 14.3 & 7.5 & 9 . \\ 1896 . & 16.9 & 14.2 & 8.3 & 7.9 \\ 1897 . & 17.2 & 14.1 & 8.6 & 8.1 \\ 1898 . & 17.4 & 14.1 & 9.7 & 7 . \\ 1899 . & 20.6 & 17 . & 11.7 & 7.6 \\ 1900 . & 19.8 & 15.9 & 12.7 & 6.2 \\ 1901 . & 16.9 & 12.3 & 11.2 & 4.8 \\ 1902 . & 19.1 & 14.2 & 11.8 & 6.6 \\ 1903 . & 20.7 & 15.2 & 13.1 & 6.9 \\ 1904 . & 23.7 & 17.0 & 14.7 & 8.6\end{array}$

II. - La formation du grompe de la Discontogesellschaft.

La Discontogesellschaft possède anjourd'hui (fin 1904) 3 filiales (non compris la Norddeutsche Bank de Hambourg) en Allemagne: Berlin. Francfort (1900), Brême (1904), par absorption de la maison J. Schultze et Wolde; 1 à Londres depuis 1900.

Elle possède 7 caisses de dépôt à Berlin.

$$
2 \text { - - à Charlottemburg }
$$

et commandite la maison E. Tornquist et Cie à BuenosAyres depuis 1889, et la maison A. de Bary et Cie à Anvers, pour un montant de $2.187 .000 \mathrm{M}$.

La Discontogesellschaft a fondé ou concouru à la fondation des socirtés filiales suivantes:

1880. La Deulsche Handeis und Planlagengesellschaft der Südseeinselı à Hambourg, au capital de 2.7500.000 M. 
1\$87. La Brasilianische Bank fïr Dentschlamel it Hambourg, au capital le $10.000 .000 \mathrm{M}$.

t filiales au Brésil.

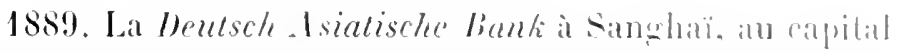
de 8.000 .000 tads, avec le comenurs des aulres mrambor banques allemandes.

7 liliales.

1894. La Bancu Commerciale Italinua ì Yailand, an catpital de 80.000 .000 lires.

2:3 filiales en thalic.

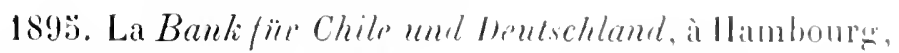
all capital de $10.000 .000 \mathrm{M}$.

7 liliales, Valparaiso, Santiago, Concepeion, Temuer, Antofagasta, La Paz, Oruro.

1s97. La Bunca Generale Romenme, a linkitrest:

1. liliales.

1898. La Banune Intemutionale de Timurelles.

1904. La Deutsch Ostuprikanische Bunk, au capilal de $2.000 .000 \mathrm{M}$.

190:3. La Bayerische Disconto und Wechsel Bank te Nïremberg (alpital 12.000.000 M.) par la fusion des mai-

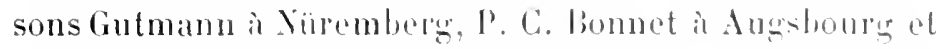

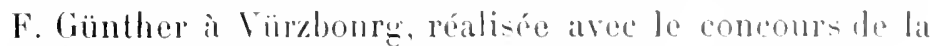
Bayerische Ilypoteken und Wechsol Bient de Miinich.

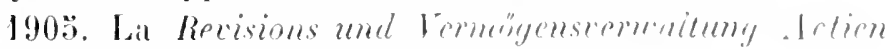
gesellscherfe à Berlin, ald caprital do 1.000.0000 M.

Constitution du groupe de la Discontogesellschaft.

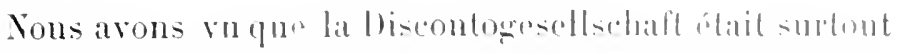

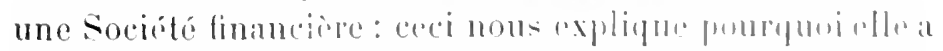
conmencési tard son mouvement dexpinsion et de dil- 
fusion à travers les provinces; une banque d'affaires qui doit conduire la majorité de ses opérations avec ses propres capitaux tend en effet plutôt à la centralisation; si l'on excepte la Norddeutsche Bank de Hambourg dont l'absorption fut, comme nous l'avons vu, due à des causes un peu spéciales, la Iiscontogesellschaft ne possédait encore en 1900 ni une filiale ni une caisse de dépôts. Mais depuis cette époque, elle a changé de politique et rapidement regagné le terrain perdu en assurant sa participation, par possession d'actions, dans quelques banques régionales, peu nombreuses sans doute, mais fort importantes et nettement décentralisćes, - comme aussi en poussant à la fusion de banques privées entre elles et à leur transformation en Sociétés par actions placées sous son contròle.

A) La Discontogesellschaft a absorbé les maisons : en 1900, Rothschild et fils à Franefort; en 1903, Schulze et Walde à Brême, tranformées en filiales.

B) Participations par possession d'actions.

a) En 1895, la Discontogesellschaft porte son capital de 75 à 115 millions de marks et échange les 10 millions de marks d'actions de la Norddeutsche Bank de Hambourg contre ses 40 millions de marks de nouvelles actions qu'elle garde d'ailleurs en totalité par devers elle. La Norddeutsehe Bank est devenue en fait une pure filiale de la Disconto: son capital a été porté en 1904 à $50.000 .000 \mathrm{M}$. Réserves en 190วั: 10 millions M. environ.

La Norddeutsche Bank possède :

1 filiale, une caisse de dépòt, 1 commandite et a absorbé en 1904 la maison W. S. Warburg, à Altona.

b) La Discontogesellschaft possède depuis $1901, \check{5} .540 .000$ 
marlis d'actions w. l'Allgemeine Deutsche Credit Ans-

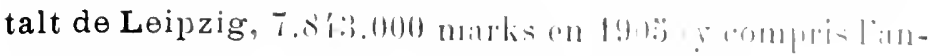

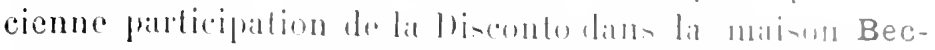
ker et Cie fusionnin (n) 1!)! avo: l'Allgemeine Deutsche Credit Anstalt.

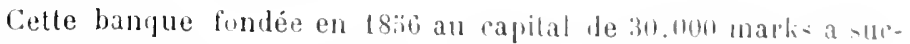
cessivement porté son capital.

en 1891 it 42.000 .0010 matris.

en 1897 à

en 1899 à $60.00 m .0041$

en 1901 à 7 ro.000.040

en $190 \%$ a 75.500 .000

(plas 30.700.0100 " de réserve-

Elle possède \& liliales.

11 caisses de déprits.

1 commandite.

Elle a successivement absorbé les maisons:

1901, Becker et Cie, il leiprig.

1900, C. Blaufuss, di liéria.

1903, Güntlier et Rodolph, ì llecile.

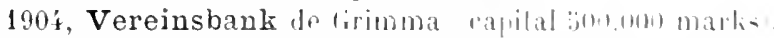

Elle possède elle-mème par possession dactions, des parti. cipations dans

la maison Borne Busse et Cie. I linlin. apital b. $1001.11+10$ marlis.

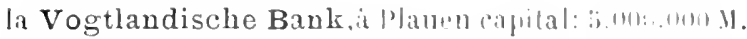

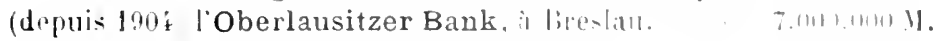

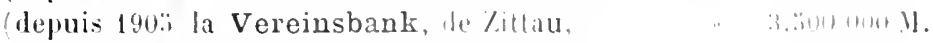

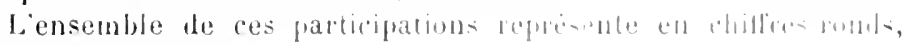
13.000 .000 marks.

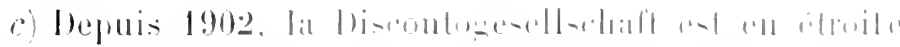

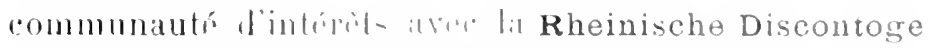

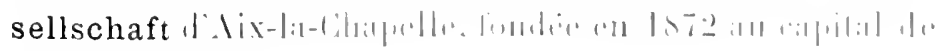
60.0000 mirkis.

1883 capilal.

(111).11111)

las: -

$1 ! 11111.111111 \cdot 10$ 
En 1894 à 13.500 .000 marks.

En 1898 à 18.500 .000 "

En 1902 à 35.000 .000 "

En 1905 à 44.600.000 " dont 3 millions de marks d'actions environ possédés par la Discontogesellschaft.

La Rheinische Disconto possède:

6 filiales.

5 caisses de dépôt.

2 commandites.

Elle a successivement absorbé :

1901. La Bank für Rheinland und Westfalen, à Cologne

(capital 8.000.000 marks).

1901. La Coblenzer Bank (capital 2.000.000 marks).

1902. La maison Groethuysen et Linxweiler, à Viersen.

1904. La Kolnische Wechsler und Commissionsbank

(capilal 12.000.000 marks).

1 commandite.

1 filiale.

Elle est intéressée depuis ces dernières années, par possession d'actions :

à la Bochumer Bank (capital 5.000.000 marks) qui absorba elle-ınème en 1897 la maison Hüttemann Korte.

2 filiales.

à l'Eschweiler Bank (capital 5.000.000 marks).

à la Dürener Bank (capital 6.000.000 marks) qui a ellemème absorbé l'Euskirchener Volksbank.

à la Volksbank Geilenkirchen (capital 315.000 marks).

à l'Oberbergische Bank à Ohl (capital ว00.000 marks).

à l'Eupener Creditbank (capital 500.000 marks).

au Neuwieder Bankverein (capital 200.000 marks).

d) La Discontogesellschaft possède enfin depuis 1904 , 2 participations: la première dans le Barmer Bankverein, Hinsberg Fischer et Cie à Barmen fondé en 1867.

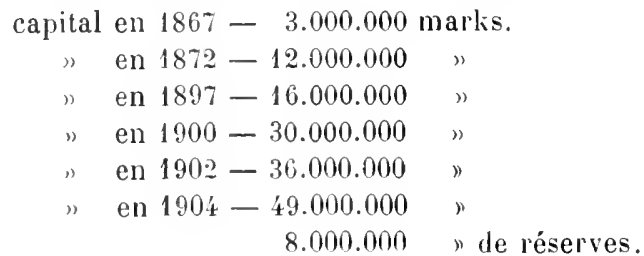


Cette banque possède 12 filiales el caisses de dépits. lille a successivement absorbé :

en 1898 la maison Quark et Cie, à M. liladbach.

en 1900 Leffmann Stern, à llagen.

en 1902 le Düsseldorfer Bankverein aucapitalileg(n). (um I).

en 190' le Dortmunder Bankverein déjà intéres-é lal. mème à la maison Balck et lie.

$2^{\circ}$ La seconde dans la Süddeutsche Discontogesellschaft. née de la fusion des maisons E. Latrubures, il Francfort et W. E. Ladenburge tils, a Jannlreim, ot de lour transformalion en Société par actions au capital de 20.(1010.1100) marks,

1 filiale.

1 commandite.

L'ensemble des participations de la lliscontugasellschaft dans les banques secondaires représentait en 1901 . ૧ŏ.210.000 marks; elle délìgue un administrateur all conseil de ces différentes banques rinuméries: colles ci sont représentées is leur tour au conseil de direction de la Discontogesellschaft.

Le tableau suivant, des diridendes distribués par les principales banques aftiliées au groupe dir la llisonnto. gesellschaft montrera les bónóbices que cethe derniere a pu retirer de ses participations.

Norddeutsche 1895-1890 1897-1843 $1899.1900 \quad 1901 \quad 1902 \quad 1943 \quad 1901$

Bank.

Allgemeine Deutsch

Credit . . . .

Mheinische Dis-

contog...

Barmel Bankve-

rein....
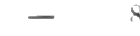

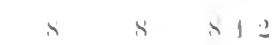

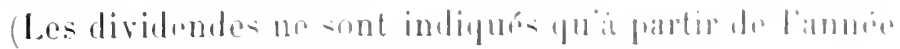

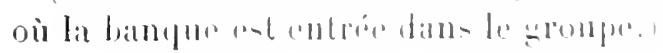


III. - Les participalions industriplles et commereiales de la Discontogesellschaft. - Les affaires consortiales.

Malgré toutes les critiques que lui a maintes fois adressées à ce sujet la presse financière (1), la Discontogesellschaft n’a jamais consenti à distinguer dans ses bilans et rapports le chapitre de ses opérations consortiales de celui des valeurs qu'elle possède en propre, pas plus qu'à donner le moindre renseignement sur la composition de son portefenille-titres. Nous devrons donc, forcément, nous en tenir aux généralités.

La Disconto a surtout consacré son activité aux grandes affaires internationales (entreprises industrielles ou chemins de fer). Elle a réorganisé en ces dernières années la Dortmunder Union et participé à la fondation de la $A s$ cherslebener Maschinenbau Actien Gesellschaft, de la Niles Werkzeugmuschinenfahrik, de la Maschinon fubrik Luther, à Brunswick (1898) - comme aussi aux fondations de la société des Chemins de fer Vénézuéliens et de la Compagnie parisienne d'air comprimé et d'électricité, de la Compagnie coloniale (1899), de la Rumanische Petroleum Actiengesellschaft (1904).

Nous donnons la liste des sociétés dans le conseil d'administration desquelles la Disconto délègue soit un directeur, soit un de ses gérants:

10 Mines et charbonnages.

Union Actiengesellschaft für Eisen-Bergithan und Stahlindustrie, à Dortmund.

(1) Störungen..., p. 147. 
Bochïmer Bergwerks Actiongesellschlaft, it Bochum.

Gelsenkirehen Bergwerks Actirngesellechaft, it reloenkirchen.

Otavi-Minen med Eisenbalnu, Cesellschaft.

Schantung Bergbau Gesellschaft at Tsingtatu.
"
1) à Berlin.

Actien Gesellschaft Schalker Gruben und Ilättenverein, a Gelsenkirchen.

Vereinigte Kö̈nigs mul Laura Ilïtten Actiengesellschaft fïr Bergbau und IIïttenbetrich, is Berlin.

Actiengesellschalt für Berghan Eisin und Stahlfalmikation.

A. G. Centrale für Bereweren Francfort.

Phönix 1. G. für Beroban mal Hätlenbetrieb (Saar).

Rheinische Stahlwerke Meiderich).

$2^{\circ}$ Entreprises de mansport.

IIamburg Amerikanischelack etfaht Actiengesellschalt.

Osteuropäische Telegraphen Gesellschaft.

Dortmund-Gronan-Enseheder Eisenbahn Gesellschalt.

Allgemeine Berlinen omnibus Action libsellidhaft.

Grossen Leiprigar Strasenbalan.

Warschau Wiener Eisenbahn Gesellschallt.

\section{Irabriques de machines.}

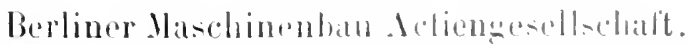

Ascherslebener Naschimenban Actiongeroll-chath.

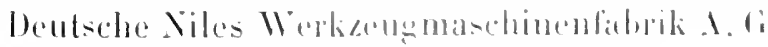

Masehinenfalmik und lian-Anstalt L,ullur.

\section{to Entreplises direrses.}

Guinea Lompany, a burlin.

Compagnie Internationate derint. 
Ohlendorffsche Guanowerke, Hambourg.

Tuchersche Brauerei A. G., Nuremberg.

$5^{\circ}$ Industries électriques.

Allgemeine Elektricitätsgesellschaft, Berlin.

Deutsche Ueberseeische Elektricitäsgesellschaft.

Elektrische Strassenbahn, Valparaiso.

Gesellschaft für elektrische Unternehmungen, Berlin, Internationale Elektricitätsgesellschaft.

Nous avons dit que la Discontogesellschaft, membre influent du consortium Rotschild, du consortium de Prusse et de celui des emprunts russes avait pris part à presque toutes les émissions d'emprunt de l'Autriclıe, de la Hongrie, de la Russie, de la Roumanie, de l'Argentine et du Chili. On trouvera (p. 164) la liste des entreprises transatlantiques et asiatiques à la fondation desquelles elle a participé. Le tableau suivant extrait du rapport général de 1902, montrera l'activité de la Disconto sur le terrain des émissions.

Elle a pendant cette année 1902 parlicipé aux négociations des émissions suivantes:

Emprunt 40/0 de la ville d'Elberfeld.

" $30 / 0$ de l'Empire allemand.

" $3120 / 0$ consolidé de l'Etat prussien.

" $31 / 20 / 0$ des chemins de fer bavarois

" $31 / 20 / 0$ de l'Etat Bavarois.

" $31 / 20 / 0$ de la ville de Stettin.

" russe $40 / 0$.

" $31 / 20 / 0 \mathrm{du}$ Pfälzische Ludwigs und Nordbahn.

Emission des nouvelles actions de la Gelsenkirchener Bergwerks Actiengesellschaft. 
Emission des nouvelles actions de la Bank lïr llandol und ludustrie.

Emission des nouvelles actions de la kieler bank.

Emprunt \& 00 du Lreisgemoinde Vittelfranken

" 400 de la ville de IIalle.

" 31200 du Wurttembere.

" $\quad 4200$ (obligations) du Maphta Productions Gesellschalt Nobel frères. à Sint-Póterboure.

Emprunt 31200 du Graml Duche de Barde.

"1 0 , 0 conversion du royame de llongrie.

" $\quad 31200 \mathrm{de} \mathrm{la} \mathrm{ville} \mathrm{de} \mathrm{lioniesterer.}$

Emission des nowelles actions du Yorkleutscha Lloyd, à Brìme.

Emission des nouvelles actions du llambure-Amerikitnische Packetfahrt Gesellichaft, it llamboure.

Emprunt $30 / 0$ de l'Etat Saxon.

41200 (obligations fonciores de la BasaltActien Giesellschaft it Linz-sur-le-Rhin.

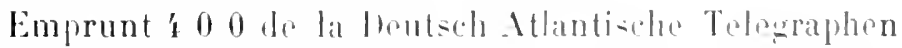
Actiengesellschaft, à Cologne.

Emprunt to 0 obligations de la Nordentsche Lloyd, à Brême.

Emprunt 31200 (obligations) du grand ducal Landes Credit Anstalt, is frotha.

Emprunt \& 1200 obligation-dela Lamblbak a berlin.

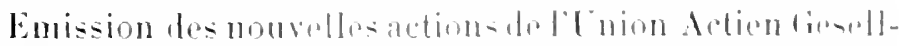

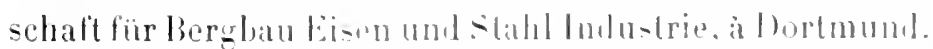

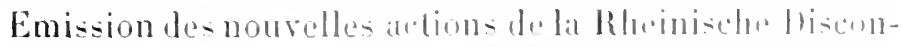
togesellschalt.

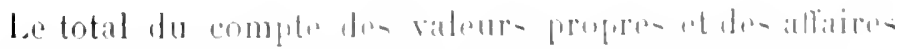

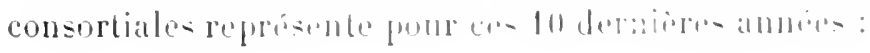


17'. DEUXIÈME PARTIE. - ChAPITRE PREMIER

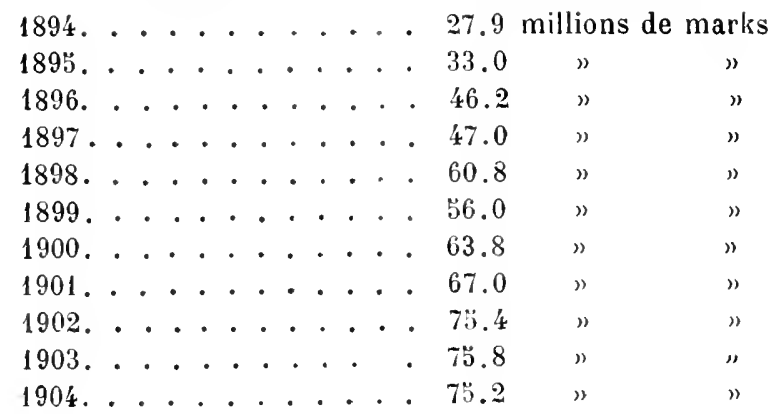

Soit pour 1904, plus de $100 / 0$ du total de l'actif et plus de $300 / 0$ des ressources propres de la banque. On voit l'importance qu'ont prise ces chapitres du bilan dans l'activité de la Discontogesellschaft et l'influence de cette dernière dans la vie économique générale. 


\section{CHAPITRE II}

LA BANK FÜR HANDEL UND INDUSTRIE.

1. - Le developpement propre de la Bank lïr Ilandel und Induslrie.

La Bank für Handel und Industrie - on plus brievement la Darmslädter Bank - fut foudce en $15: 303$ a Darmstadt au capital de 203 nullions de llorins. Cótait, at-t-on dit, "lessai d'ateclimatation on Allemagne d'une plante liançaise s (1), autrement dit, les fondateurs s'inspirèrent du Crédit Mobilier des Perpire et roulurent créer und banque, que nécessitait le déroloppenent áconomique du pays. oì

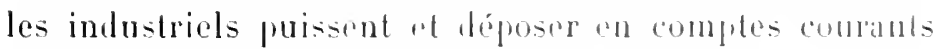
portant intérêts leurs capitanx disponibles at trouver les sommes nécessaires a lextension de leur activite.

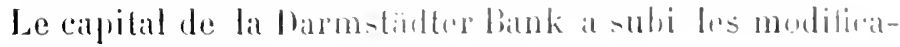
tions suirantes:

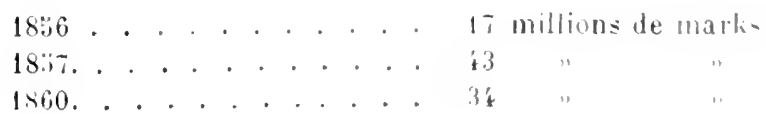

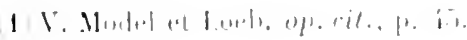




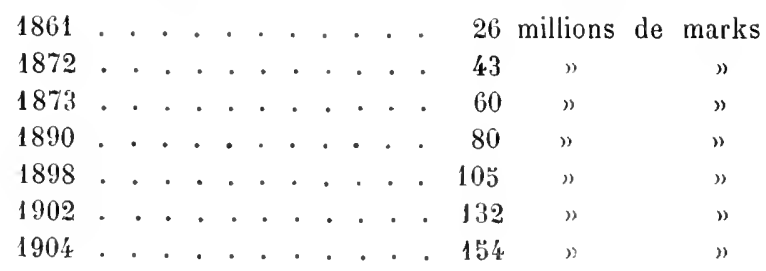

L'examen des bilans de la Bank für IIandel und Industrie nous permet de contrôler les principaux faits suivants :

$11^{\circ}$ La progresssion de l'activité générale de la banque ne s'affirme guère qu'à partir des années 1870-1871; l'ensemble des opérations a diminué durant la période qui précède ces années et la banque a dû consentir à une réduction de son capital-actions.

$2^{\circ}$ A partir de 1870 , nous pouvons constater avec un accroissement de l'activité générale de la banque un certain développement du reguläre Geschäft,coïncidant généralement avec une période de crise ou d'inactivité industrielle (1867-1873). Le développement de cette branche d'affaires est surtout remarquable à partir de 1901 ; il a été singulièrement favorisé par l'absorption de la Bank für Süddeutschland et l'alliance avec la Breslauer Discontobank.

$3^{\circ}$ La distinction des bénéfices en bénéfices provenant des affaires régulières de banque et des bénéfices provenant des titres et des affaires en participation non moins que le chiffre élevé de ces dernières (col. 9 à 11 de l'actif) montre que la Darmstïdter Bank est surtout une banque d'affaires. Rien d'étonnant par conséquent à ce qu'elle ait plus souffert que certaines de ses concurrentes des crises économiques. On remarquera l'irrégularité des bénéfices cl les dépressions des années 1857, 1891, 1901. 


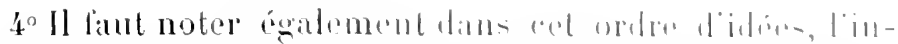
suflisance des chitfres inserits allx cumerlisserments al. 1.2

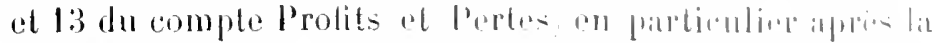

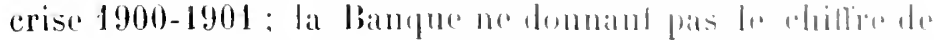
ses engagements dans les dillípentus alfarireson! pant donter que les pertes éprouvées par elle sojont complibument amorties.

$5^{\circ}$ Remarquons enfin que les angmentations sulderives

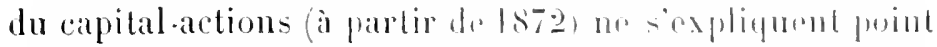
par une alogmentation parallidedes diprits ret comples cone-

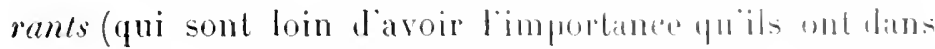
nos grands établissements de crédit on mime it la Denlsche Bank; ces chapitres sont composés dans mor proportion à peu pres égale de dépots à rue de de comples a

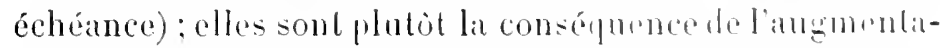
tion dans les crédits aceordés - junr une larere part is découvert - dansles participations ot les aftaires en (unt) sortium comme aussi dans le chifle des commandites de

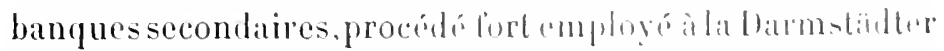

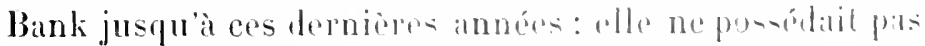

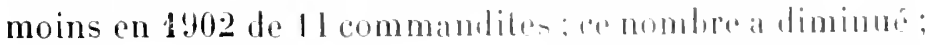
la banque use aujourd hui du procéde diextension frat achat ou échange des actions \$ 11 .

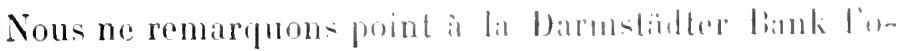

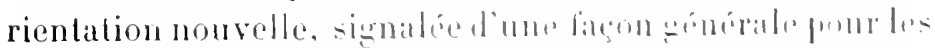

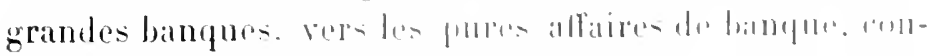

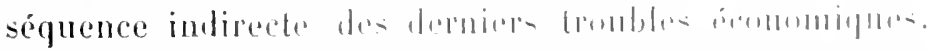

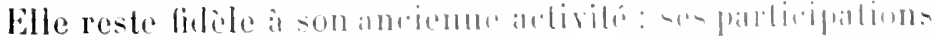

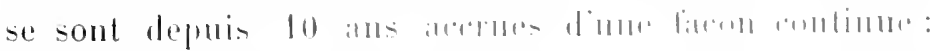

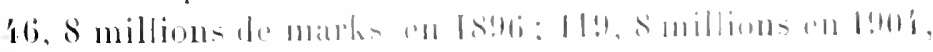

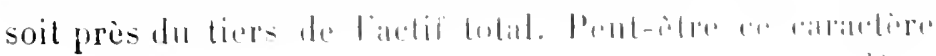


particulier vient-il nuire à la diffusion mème de la banque; les banques d'affaires ont peu à gagner d'une extrême décentralisation, et de fait nous la voyons tenir le quatrième rang dans le concert des groupes de banques; de jeunes et ardentes rivales comme la Commerz und Disconto Bank de Hambourg qui vient de fusionner avec la Berliner Bank la dépasseront bientôt peut-ètre par le nombre de leurs succursales ou de leurs participations. Mais à d'autres points de vue, l'industrie allemande toute entière doit se féliciter de posséder un tel établissement qui mit toujours à sa disposition le crédit sous ses formes le plus variées. On a pu dire de la Darmstädter Bank qu'elle avait parfaitement mérité son nom de "Banque pour le Commerce et l'Industrie." 
Darmstædter Bank Millim-d, malk IS., In!:

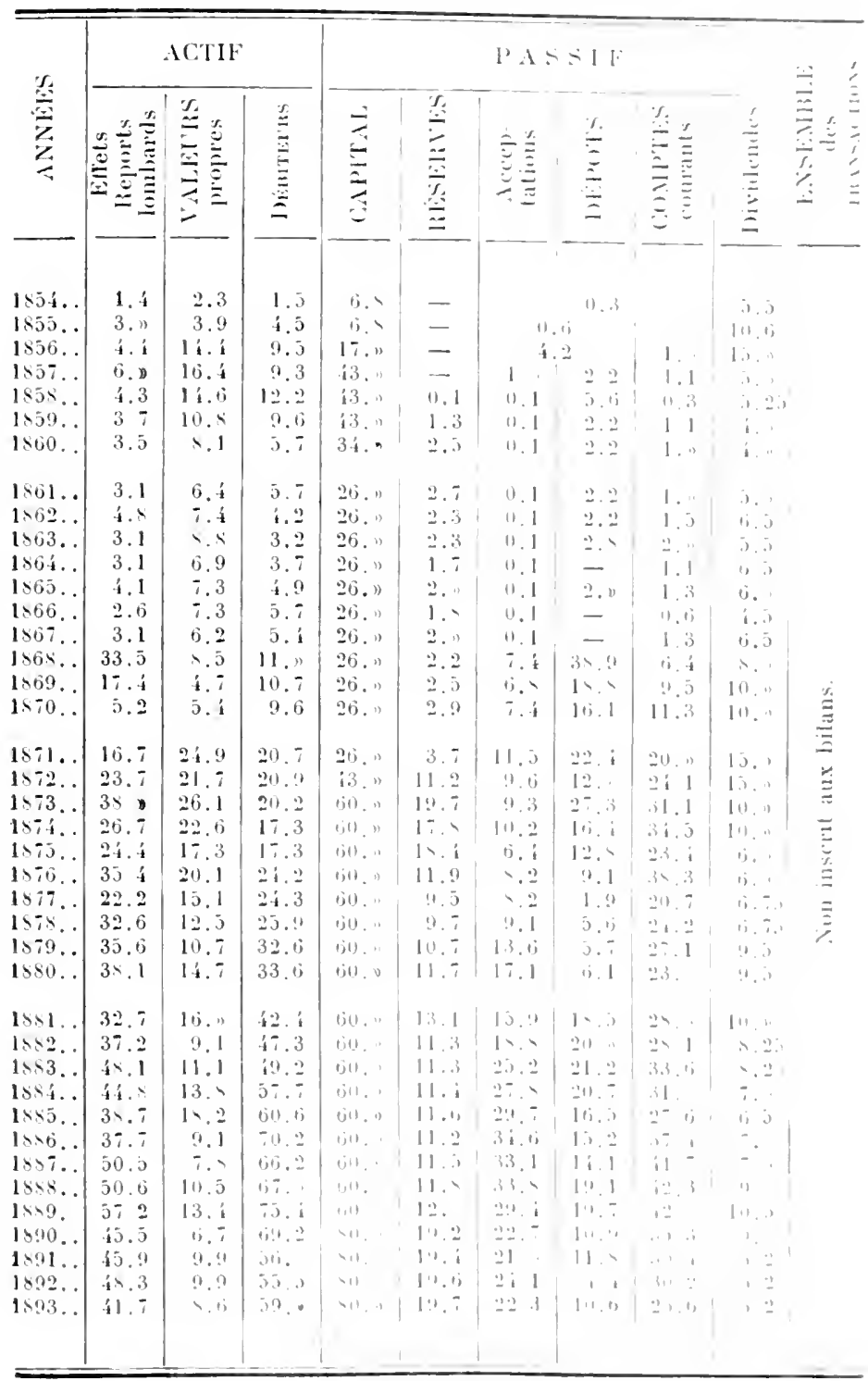




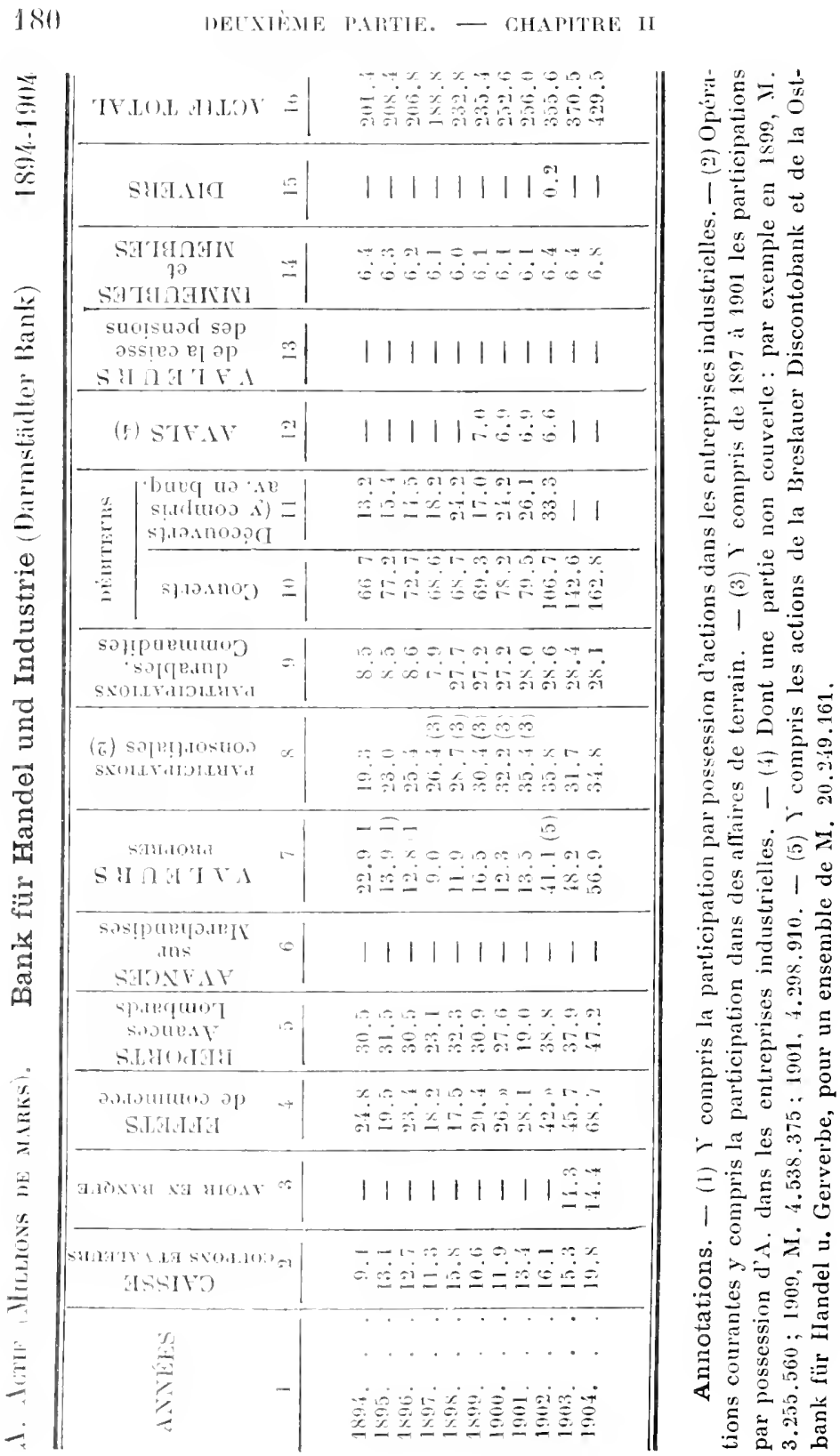




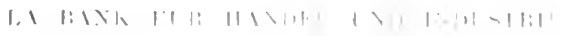

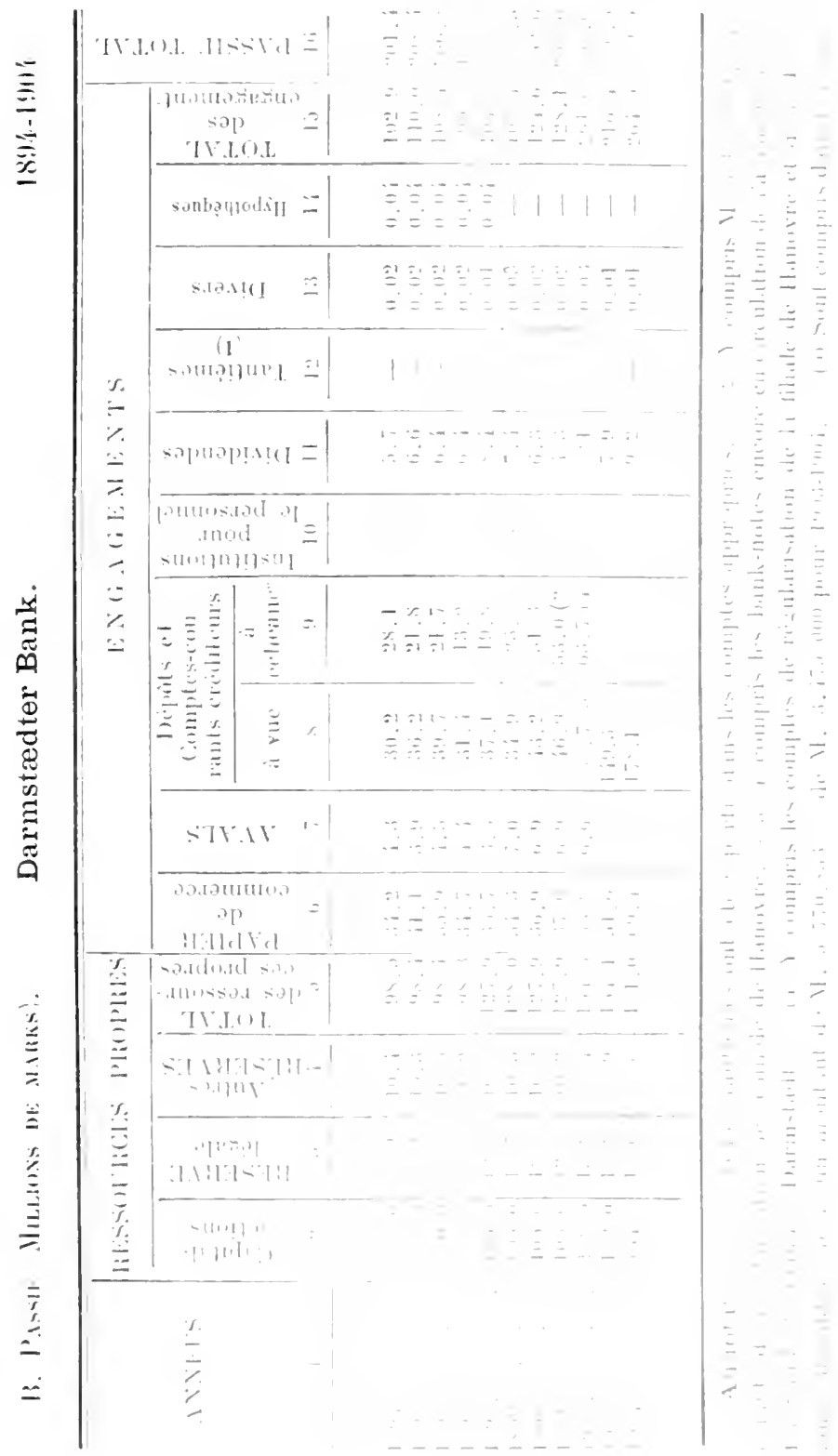




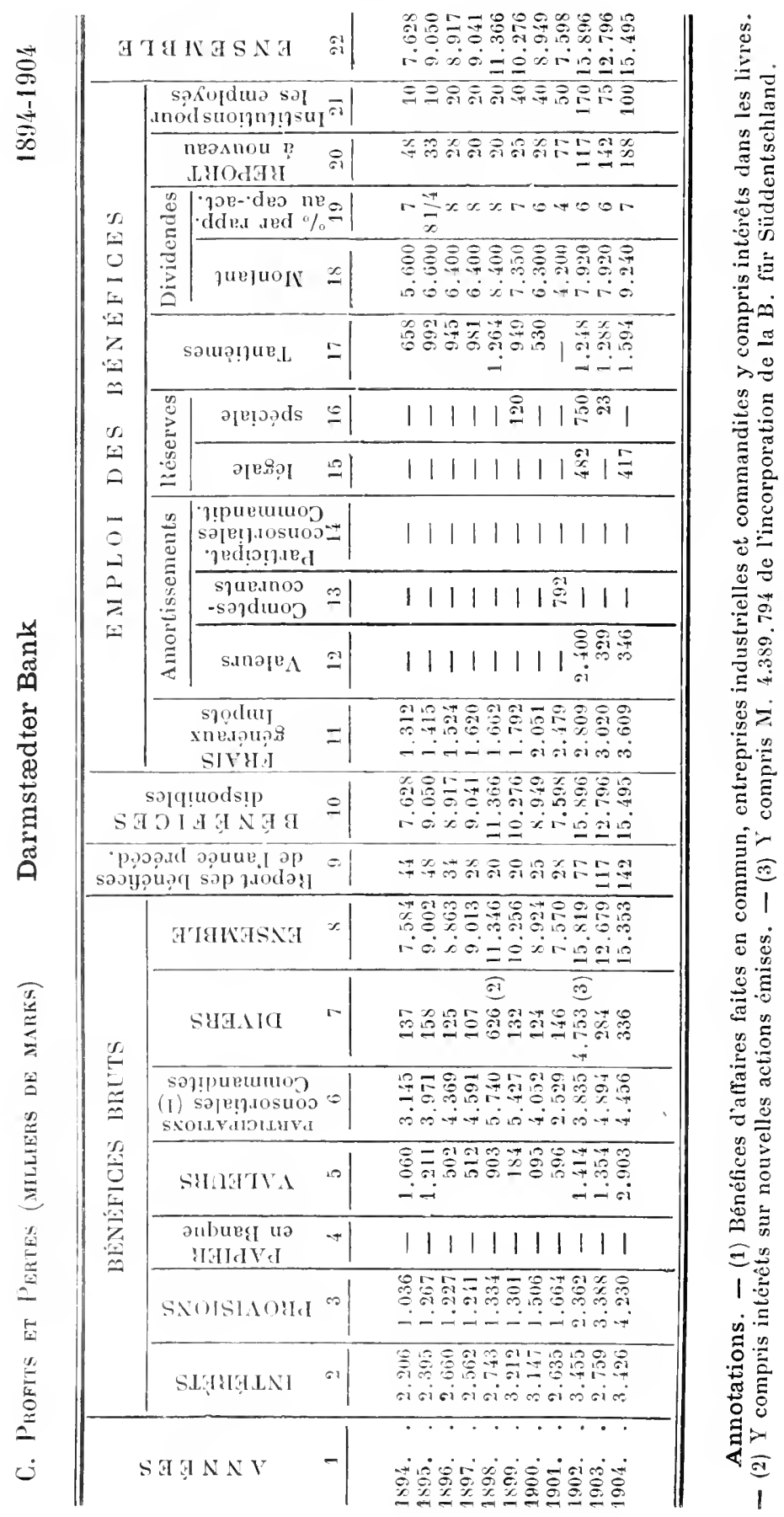




\section{Darmstaller Bant.}

Bénéfices (millions de marks).

\begin{tabular}{|c|c|c|c|c|}
\hline Annees & Bénefice brut & Bénefice net & $\begin{array}{l}\text { Produit des } \\
\text { atlaires de } \\
\text { hanque ré. } \\
\text { grulieres }\end{array}$ & $\begin{array}{c}\text { Proiduit des } \\
\text { titres el } \\
\text { fanticipation }\end{array}$ \\
\hline 1854. & 0.8 & 0.3 & & \\
\hline $18: 3$. & 1.5 & 1.3 & & \\
\hline 1856. & 3.9 & 3.6 & 2.2 & 1.7 \\
\hline $18: \%$ & 2.8 & 2.1 & 2.3 & 0.3 \\
\hline 18308. & 2.8 & 2.3 & $2 . .3$ & 0.3 \\
\hline $180 \ddot{9}$. & - & - & & \\
\hline 1860 & 4.2 & 1.8 & 1.7 & 2.3 \\
\hline 1861 & 2.5 & 1.3 & 1.5 & 1. \\
\hline 1862. & 3.3 & 2.1 & 1.8 & 1.5 \\
\hline 1863. & 2. & $1 . \tilde{z}$ & $1 . i$ & 0.3 \\
\hline 1864 & 2.3 & 1.8 & 1.8 & $0 .$. \\
\hline $186 \ddot{.}$ & 2.3 & 1.9 & 1.8 & $11 . ;$ \\
\hline 1866. & 2.2 & 1.4 & $\therefore$ & 0.2 \\
\hline $186 \pi$ & 2.6 & 2.1 & 1.8 & 0.8 \\
\hline 1868. & $3 . t$ & 2.9 & 2.4 & 1. \\
\hline 1869 & 4. & 3.2 & 2.9 & 1.1 \\
\hline 1870. & 3.8 & 3.3 & $2 . \tau$ & 1.1 \\
\hline 1871. & 8.6 & $\tau .2$ & 3.7 & +.9 \\
\hline 1872. & 8.7 & 8. & $\vdots .1$ & 36 \\
\hline 1873. & b. & 4.7 & i. 9 & 1.1 \\
\hline 1874. & 6.4 & $\therefore$. & 3.3 & 3.1 \\
\hline 187.3. & 4.3 & 11. & 3.9 & $11 . \dot{t}$ \\
\hline 1876. & 3.8 & 0.1 & 3.7 & 0.1 \\
\hline $187 \%$ & $\ddot{5 . t}$ & $\therefore$ & 3.7 & 1.9 \\
\hline 1878. & 3.8 & $i .:$ & .3 .9 & $1 . ! 1$ \\
\hline 1879. & $8 .: i$ & 6.5 & i. i & 4.1 \\
\hline 1880. & $7 .$. & $\therefore . . i$ & $i$. & 2.7 \\
\hline 1881. & 7.6 & i. $\mathrm{h}$ & $\therefore 1$ & 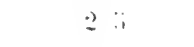 \\
\hline 1882 & 6.3 & :. : & $i \cdot i$ & 1.7 \\
\hline 1883. & 6.7 & $\therefore .:$ & $i: i$ & $\because:$ \\
\hline 1881 & 6.2 & i. i & $i:$ & $\therefore$ \\
\hline $188 \%$. & $\ddot{7.7}$ & $i . t i$ & i. i & 1 \\
\hline 1886 & ii. 1 & .1 & i. i & 1.7 \\
\hline $188 \%$ & 6.1 & 3.1 & i. & $1 . ;$ \\
\hline
\end{tabular}


Darmstïdter Banli (Suite).

\begin{tabular}{|c|c|c|c|c|}
\hline Années & Bénéfice brut & Bénélice net & $\begin{array}{l}\text { Produit des } \\
\text { affaires de } \\
\text { banque ré- } \\
\text { gulières }\end{array}$ & $\begin{array}{l}\text { Produit des } \\
\text { titres et } \\
\text { participations }\end{array}$ \\
\hline 1888. & 7.7 & 6.7 & 4.9 & 2.7 \\
\hline 1889 . & 9.9 & 8.6 & 6.3 & 3.5 \\
\hline 1890. & 9.8 & 8.4 & 6.6 & 3.1 \\
\hline 1891. & 5.9 & 4.6 & 5.4 & 0.4 \\
\hline 1892. & 6. & 4.5 & 4.9 & 1. \\
\hline $18: 3$. & 5.9 & $4 . \%$ & 5.1 & 0.7 \\
\hline 1894. & 7.6 & 6.3 & s. & 2.5 \\
\hline $189 \%$. & 10.7 & 7.6 & 6.8 & 3.5 \\
\hline 1896. & 10.6 & 7.3 & 7. & 3.2 \\
\hline 1807. & 10.4 & 7.4 & 6.7 & 3.5 \\
\hline 1898. & 13.0 & 9.6 & 8.2 & 4.5 \\
\hline 1899. & $12 .:$ & 8.3 & 9.3 & 2.9 \\
\hline 1900 . & 11.8 & 6.8 & 10.2 & 1.4 \\
\hline 1901. & 7.5 & 4.2 & 4.2 & 2.9 \\
\hline 1902. & 13.8 & 9.2 & $\ddot{3} .8$ & $9.2(1)$. \\
\hline 1903. & 12.7 & 9.3 & 6 & 6.4 \\
\hline 1904. & $1: i .4$ & 11 & 7.6 & 7.6 \\
\hline
\end{tabular}

\section{H. - La formation du groupe de la Bank fï̈r I Iandel und Industrie.}

La Bank für Handel und Industrie a successivement fondélestiliales (4) smivantes: Franclort 1864, Berlin 1871. Ilanove, 1900 (par absorption de la banque II. Oppenheimer), Strasbentg, 1901.

Elle possède ì Berlin, 11 caicses de dópôts.

$\begin{array}{lll}\text { à Stellin, } & 1 & - \\ \text { à Leifrig, } & 1 & - \\ \text { à Olfenbach, } & 1 & - \\ \text { à Giessen, } & 1 & - \\ \text { à Francfort, } & 1 & -\end{array}$

1 l' compris 4.390.00 minlss de bénéfices rẻalisés lors de l'absorp. tion de la Bank fïr Süddeutschland. 


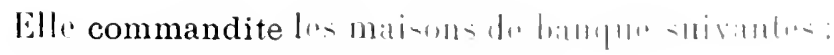

llerm. Armold el cie, it llallw.

Ed. Lab el Cir, is Nourstalt.

Rëmrelin ed Cir, à Heilbroun.

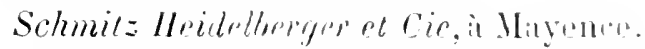

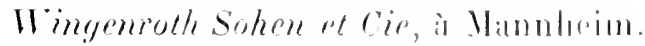

A. Kuntae ef rie a loredu

190.̈. Fuld el Cir, ì Porteim.

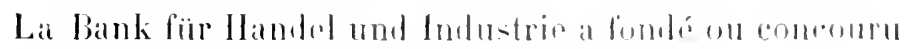
à la fondation des sociéléa filiales smivanter:

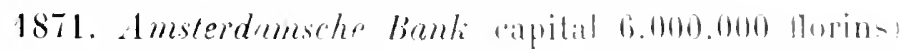
dont le conseil datministration comprend trois difions de la Darmstiadter Bank.

1851. Sïddentsche Boden Credithanti, à Mïnich.

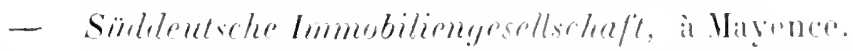

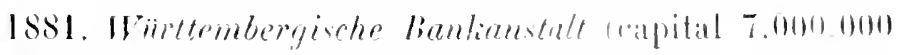

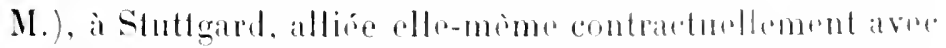
le Württembergischer Vereinsbank de Stuttgard "iphital 21.000.000 11., 6 liliales on ammanditer.

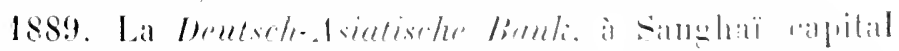

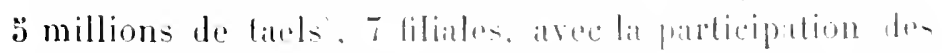

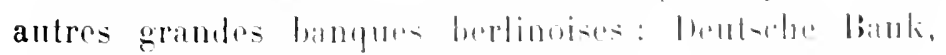

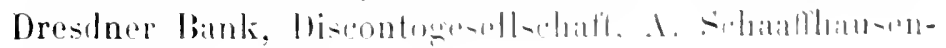
s'cher bankverin.

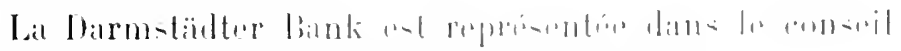

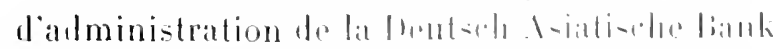

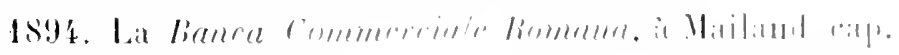

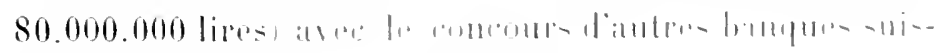

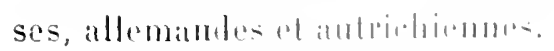

$2: 3$ liliales on llatir.

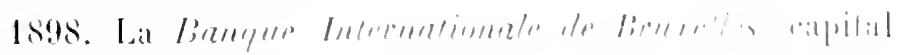

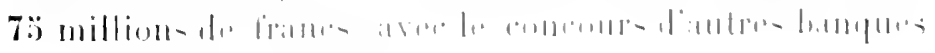

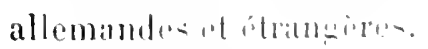


1899. La Bank für Brauindustrie, à Berlin (capital 7.000.000 M.)

La Darmstädter Bank est représentée dans le conseil d'administration de ces trois sociétés.

1900. Le Bankers Trading Syndicate, à Londres (capital 100.000 L. St.).

1904. La Banca Marmorosch Blank et Cie, à Bukarest (ancienne maison commanditée par la Bank für flandel und Industrie).

\section{Constitution du Groupe de la Darmstædter Bank.}

La Darmstädter Bank a longtemps pratiqué de préférence la politique de commandite des banques secondaires. Ce n'est qu'en ces dernières années qu'elle a procédé par voie d'absorptions et de participations par achat ou échange d'actions; cette méthode nouvelle d'extension a coüncidé avec le développement des affaires de banque proprement dites.

A) Absorptions de la Darmstüdter Banks.

1900. Gustav. Maier et Cie, à Francfort.

- H. Oppenheimer, à Hanovre.

1901. Otto Davisson, à Hanovre.

- B. Lillenthal, à Güstrow.

1902. Le siège berlinois de la Breslaner Discontobank.

- Bank für Süddeutschland, à Darmstadt (capital 14.000.000 marks). La Darmstädter Bank donna aux actionnaires 13.060.000 marks de ses propres actions.

1903. R. Varschauer de Berlin, qu'elle commanditait depuis 1898 . 
B) Participations par possesvem de teliums:

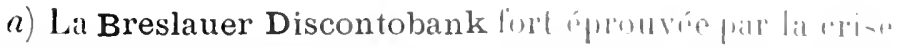

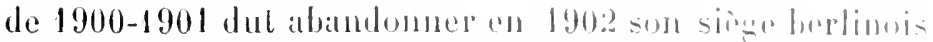

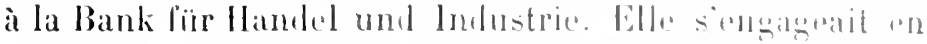

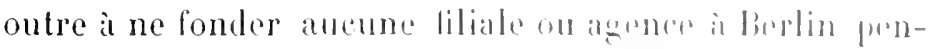
dant 10 ans, comme aussi à ne sintépesseld, directement

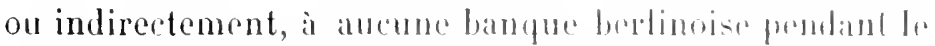
mème laps de temps; ses intérèts à licrlin devalent ètre uniquement traités par la Darmstiidler liank.

Le capital de la breslauer Discontohank qui arait sucessivement été porté à :

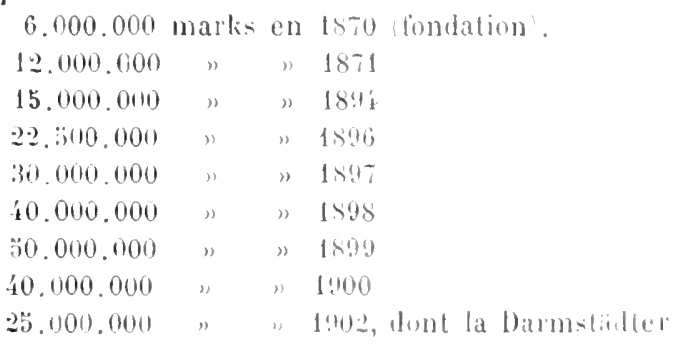

fut réduit à 40.000 .000 " " $1 ! 100$

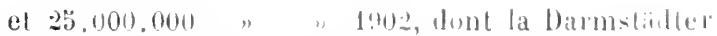

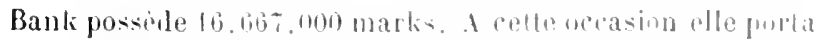
son propre capital de 10 ; i 132.0100 . (0) mark mas.

La breslauer loisconto lianli possède:

6 tiliales.

1 caisse de dépiil

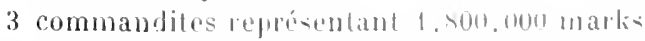

lille a successirement absorbé les maicons suivantes

1896. H. C. Plaut, a Lerlin.

1897. Friedlænder Froymark et Cie, il lierlin

1898. Ed. Calé, i linlin.

1902. Landsberger et Cie, it Katfuwitz.

1903. L. Reymann, it Ifpelı.

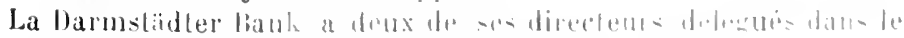

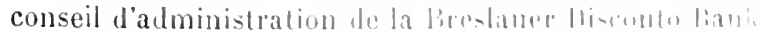

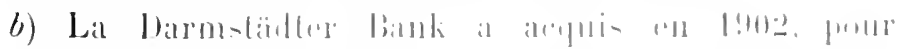

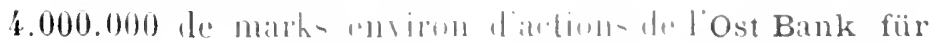


Handel und Gewerbe, de losen, fonder an 18:31 (capital 1904: $\$ .000 .000$ de marks\%。

Celle banque possède 2 liliales,

1 commandite,

6 caisses de dépots.

Elle a absorbé les maisons:

1898. Heimann Saul, à Posen.

1899. C. W. Quilitz, it Landsberg.

Elle est intéressée par la possession de 1.000.000 de marks d'actions à la Bromberger Bank für Handel und Gewerbe (capital 2.000.000 de marlis) qui a elle mème absorbé en 1901 la maison F. Lietz, à lnowrazlaw.

L'assemblée générale d॥ 12 anût 1905 a décidé de porter le capilal de l'Ostbank de Posen à 18.000 .000 de marks pour assurer l'absorption de l'ostdeustche Bank de Königsberg (ancienne maison J. Simon et fils) au capital de 10.000 .000 de markis.

c) En 1903 la Darmstäilter Bank acequiert pour 15.000 .000 de marks dactions de la Nordwestdeutsche Bank de Brême, londée en 1898 par la transformalion de la maison B. Hincke, Buthe et Meininghaus. Le capital de la Nordwestdentsche Bank a étŕ porté en 1905 de 5 a 7 millions de marks.

Celte banque possède :" liliales.

2 caisses de déprits.

Elle a absorbé en 1901 la maison Georg Meck et Cie, de Bremerhaven,

Et possède pour 120.000 marks daclions de la Lelier Bank (capital 300.1100 marlis).

C) La Durmstädter Bantic cst enfin unie contractuellement (communautés dintérìts propremont dites):

(1) Depuis 1902 it la Wechselstuben Actiengesellschaft Mercur à Vienne.

7 filiales. 


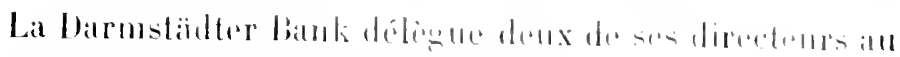
conseil dialministration de exthe hampar.

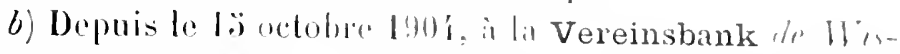

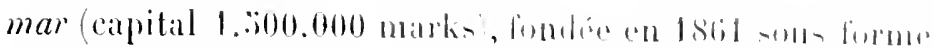
de socirté en commamblitr par atelions at tran-furmán an 1902 en Société par actions.

$\$ 9$ agences.

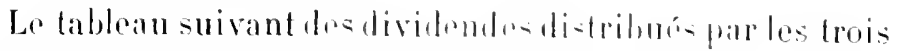
banques aftiliées à la larmstïdter liank montrora les hri-

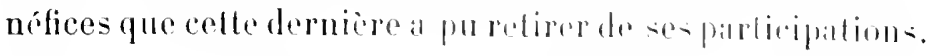

\begin{tabular}{|c|c|c|c|}
\hline & 1902 & $1: 0::$ & 1901 \\
\hline Breslauer Disconto liank : . . & & $\vdots$ & 6 \\
\hline Ostbank fur llandel und liewerbe. & i) & 6 & ii $1:$ \\
\hline
\end{tabular}

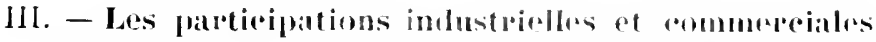

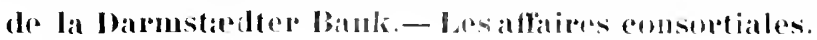

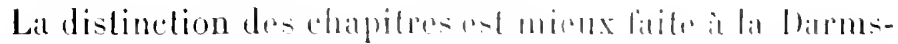

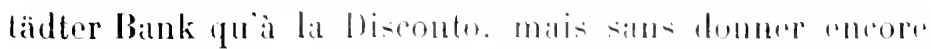

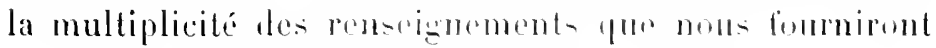

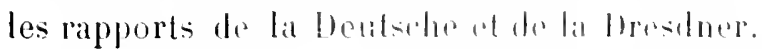

La Darmstidller, momber important du Comsortiun de

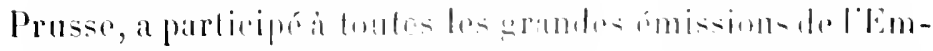

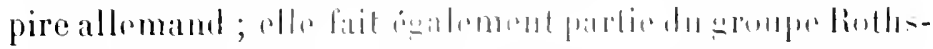

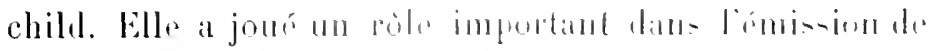

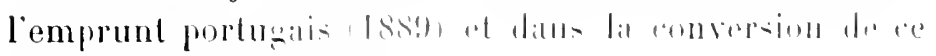
mème emprout

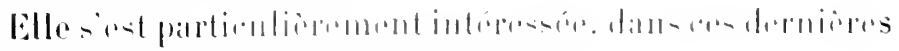

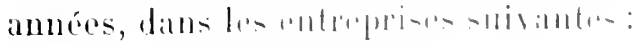

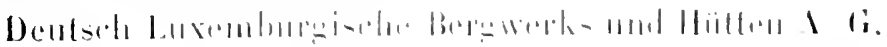


Darmstädter Immobiliengeschäft Blumenthal et Cie. Strasburger Terrain Gesellschaft.

Neue Boden A. G.

Westlische Boden A. G.

Schokoladen fabrik Gebrïder Stollwerk.

Elle délègue un de ses directeurs dans les entreprises suivantes:

$1^{\circ}$ Mines et charbonnages:

A. G. Friellicher (Linden).

Ce'ntrale für Bergwesen (Francfort).

Deutsch Luxemburgische Bergwerks und Hütten A. G. Donnersmarkhütte.

Gewerkschalt Gefrath und Sibyllagrube (Frechen). Harpener Bergbau A. G. (Dortmund). Phönix A. G. für Bergbau und Hüttenbetrieb. Riebeck'sche Montanwerke A. G. (Halle).

$2^{\circ}$ Fabriques de machnes:

A. G. Neptun Schiffswerft (Rostock). Allg. Gesellschaft für Dieselmotoren (Augsbourg). Ascherslebener Maschinenbau A. G. Ludwig Loewe et Co (Berlin). Lutz Maschinen fabrik (Darmstadt). Maschinenfabrik Ventzki (Graudenz).

$3^{\circ}$ Industries électriques:

Allg. Elektricitätsgesellschaft (Berlin).

A. G. für elektrische und Verkersunternehmungen (Buda Pest).

Elektricitäts A. G. Lahmeyer (Francfort). Gesellschaft für Elektrische Unternehmungen (Berlin). 
Leed Llektricitiolswerhe Ang-houry .

Licht mud Kraftanlagen . A. R. (Berlin. lix und Genest (Berlin).

$4^{\circ}$ Industries chimiques.

Chem. Fabrik (iriesheim. Ekektron Frantort).

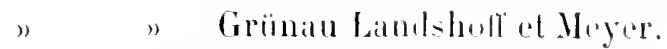

Chem. Werke H, et E. Allert Biebrich. 


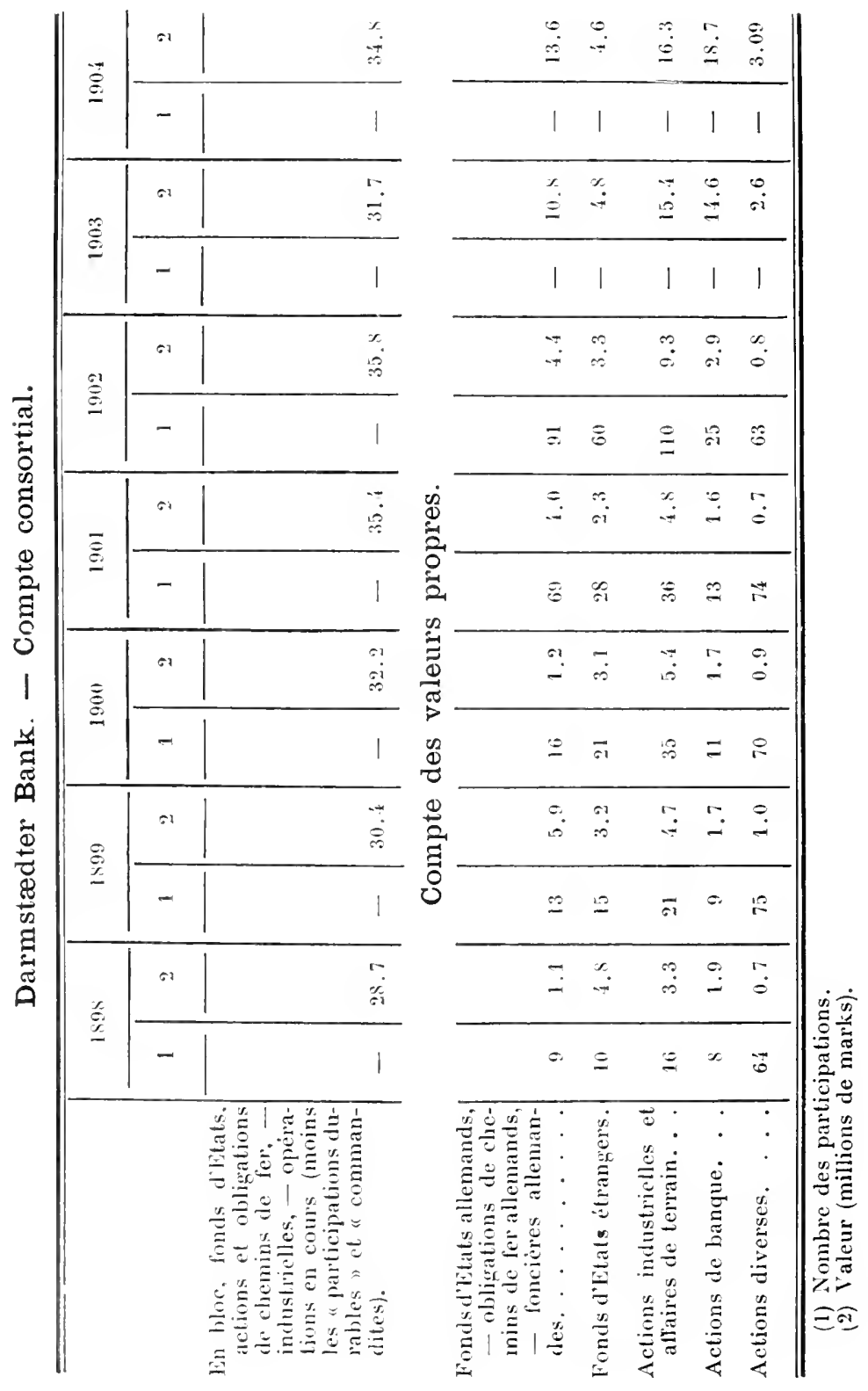




\section{CIIAPITIE III}

\section{LA DEUTSCHE BANK}

\section{I. - Le developpement propre de la Deutsche Bauk.}

La Diutsche Bank fut fondée en mars 1\$70: we fut la dernière grande banque par actions fondée sous l'ancien régime de l'autorisation gouvernementale préalable. sion but (art. 2 des statuts) est l'exploitation des alliaires de banque de toutes sortus alle doit riser particulierment au développement des relations commerciales de l. llemagne avec les pays étrangers et les marcliés d'outremer.

Son capital primitif, 13 millions de marlis, a clé poltí successivement ì

30 millions de marks. . . . en décembre 1571.

$430 "$ ".... novembre lxig.

$60 "$ " .... " 1481.

"3" $"$ mars liss.

100 " . .... novembre 1 .

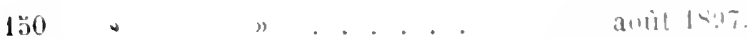

$160 "$.... derembre 1 .

$180 "$ " .....

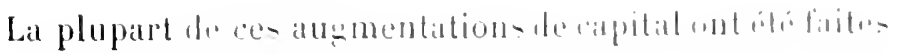


pour acquérir des participations dans les banques secondaires: nous les signalerons lors de l'examen du groupe de la Deutsche Bank.

Les tableaux suivants montreront le développement de l'activité de la Deutsche Bank : ils permettront de contrôler ces principaux faits:

$1^{\circ}$ L'activité de la Deutsche Bank a été sans cesse en augnentant et d'une manière très sensible: on remarquera l'arrêt provoqué par la crise de 1891 et celle de 1900-1901.

$2^{\circ}$ La progression des bénétices est parallèle au volume des affaires jusqu'en 1899 et les dividendes se sont élevés alors de 10 à $110 \%$; ce dernier chiffre a été maintenu pendant tonte la durée de la crise. En 1904, le dividende non encore atteint de $120 / 0$ correspond à une augmentation sensible des bénéfices bruts et nets déjà constatée pour 1903, mais la Banque maintint alors son dividende à $110 / 0$ pour créer une réserve extraordinaire de 2 millions de marks que les événements politiques (gnuerre russojaponaise) lui paraissaient rendre nécessaire.

$3^{\circ}$ La division des bénéfices en bénéfices provenant du commerce régulier de banque (intérèts, portefeuille commercial et commissions) et en bénéfices provenant des titres, des affaires en consortium et en participations durables montrera que la Deutsche Bank a inégalement développé ces deux branches de son activité.

Les affaires de banque, particulièrement les dépôts et comptes courants (col. 8 et 9 du passif) ont vivement progressé, surtout à partir de 1896 - entrée en vigueur de la loi sur les Bourses. - Les opérations comportant des immobilisations (col. 7 et 8 de l'actif) ont à peine augmenté depuis cette mène date. La Deutsche Bank semble être 


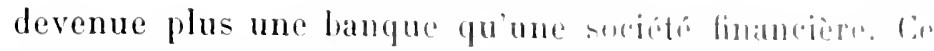
sont les bénéfices provenant des opérations do haldipll. pure qui lui ont permis de distribuer ces dividendes flevés.

$4^{\circ}$ La progression régulière des bénéfices ol,temus par lia Deutsche Bank dans ses participations montre ful alle al $\leqslant 11$ les choisir très soigneusement; clle ne paraìt point avoir subi de pertes de ce còté, lors de la crise 1!900-1901; les crises de 1873 et de 1891 nont point davantige cutravé longtemps la marche de cette branche d'exploitation.

$8^{\circ}$ Signalons la diminution progressive dans l'importance des comples débitenrs, des crédits accordís it déronvert (eol. 11 de l'actif); de mème le tolal des alceptations qui suit jusquen 1890 une marche très progressive reste à peu près stationnaire depuis 1896 ; le dórelopjement du Regulüre Bankgeschï/t semble avoir causé un souci plus grand de prudence et une plus stricte observalion des méthodes rigoureuses de banque.

$6^{\circ}$ Remarquons enfin comment la Jentsche biank a su concilier l'extension de ses progrès en Allemagne arer le but spécial quielle s’était proposé à saroir he liveloppement du conmerce d'outre-mer; ses participations dians les banques transatlantiques et asiatiques anl pris continuellement une importance plus considérable. Lianse de la Deutsche Bank fournit it cect buard "lat dímonstrittion des services que laudministration vigoureas al intel-

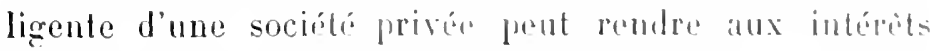
généraux du pays, tout en gagranat des dividendes mémunérateursponr ses aldionnaires 1."

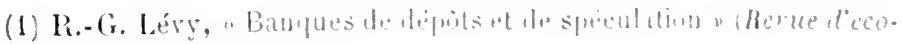
nomie politique, mai 1903 ). 
Millions de marks. Deutsche Bank

1870-1894

\begin{tabular}{|c|c|c|c|c|c|c|c|c|c|c|c|c|}
\hline \multirow{3}{*}{$\begin{array}{l}c \\
y \\
z=1 \\
z \\
y \\
4\end{array}$} & \multicolumn{5}{|c|}{ A C T I F } & \multicolumn{6}{|c|}{ PASSIF } & \multirow{3}{*}{ 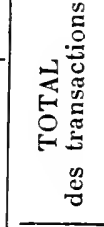 } \\
\hline & \multirow{2}{*}{\multicolumn{2}{|c|}{ 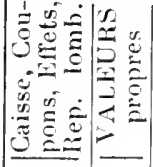 }} & \multirow{2}{*}{ 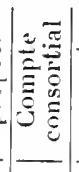 } & \multicolumn{2}{|c|}{ Débiteurs } & \multirow[b]{2}{*}{$\stackrel{\vec{s}}{\stackrel{\overrightarrow{\mid}}{\vec{U}}}$} & \multirow[b]{2}{*}{ 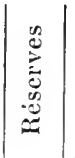 } & \multirow[b]{2}{*}{$\begin{array}{l}\dot{\vec{J}} \\
\stackrel{0}{0} \\
\dot{U} \\
\dot{U}\end{array}$} & \multirow[b]{2}{*}{$\frac{n}{a}$} & \multirow{2}{*}{ 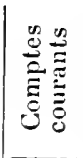 } & \multirow{2}{*}{ 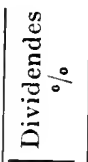 } & \\
\hline & & & & $\vec{\Xi}$ & $\mid \begin{array}{ll}1 & \infty \\
0 & 0 \\
0 & 0 \\
0 & 0 \\
2 & 0 \\
\end{array}$ & & & & & & & \\
\hline in : & 4.9 & 0.7 & - & 3.2 & 2.1 & 15 & 0.03 & 2.4 & 0.1 & 2.3 & 5 & 2390 \\
\hline $1871 \ldots$ & 21.9 & 1.1 & 0.8 & 11.7 & 7.8 & 30 & $0.16 \mid$ & 7.6 & 8.4 & 14.5 & 8 & 9514 \\
\hline $1872 \ldots$ & 37.9 & 3.2 & 1.7 & 27.8 & 18.2 & - & 0.7 & 23.5 & 8.9 & 29.8 & 8 & 2.8912 \\
\hline 1873. & 69.2 & 3.6 & 1.8 & 25.1 & 12.4 & 45 & 1.3 & 30.2 & 7.1 & 43.6 & 4 & 3.7651 \\
\hline 1874. & 76.5 & 4.9 & 1.0 & 17.5 & 17.4 & - & 2.3 & 37.6 & 10.9 & 46.1 & 5 & 5.5091 \\
\hline 1875. & 62.1 & $10 .$, & 2.4 & 24.5 & 17.0 & - & 3.4 & 42.4 & $12 .$, & 31.5 & 3 & 5,5125 \\
\hline $1876 \ldots$ & 99.0 & 11.3 & 1.7 & 35.3 & 16.3 & - & 4.4 & 41.0 & 13.5 & 82.9 & 6 & 7.1324 \\
\hline $1877 .$. & 60.7 & 4.4 & 1.2 & 41.3 & 13.4 & - & 4.8 & 38.8 & 10.1 & 31.4 & 6 & 7.3252 \\
\hline 1878. & 67.4 & 6.1 & 3.7 & 42.7 & 13.1 & - & 5.4 & 44.0 & 9.3 & 39.2 & $\begin{array}{ll}6 & 61 / 2\end{array}$ & 7.1298 \\
\hline $1879 \ldots$ & 88.1 & 4.5 & 2.9 & 56.0 & 14.1 & - & 6.6 & 48.2 & 12.1 & 56.3 & 10 & 8.8347 \\
\hline 1880. & 79.7 & 6.1 & 6.9 & 49.4 & 16.3 & - & 7.7 & 45.8 & 12.8 & 51.2 & $101 / 2$ & 10.484 \\
\hline $1881 \ldots$ & 91.7 & 19.2 & 14.3 & 64.2 & 21.2 & 一 & 9.3 & 54.2 & 14.4 & 78.1 & 10 & 12.8989 \\
\hline $1852 \ldots$ & 97.0 & 9.2 & 14.7 & 66.6 & 19.1 & 75 & 13.8 & 46.1 & 17.6 & 67.2 & 9 & 12.0 .54 \\
\hline $1883 .$. & 117,6 & 11.6 & 16.1 & 80.0 & $2 \times .0$ & - & 14.3 & 69.0 & 22.4 & 85.2 & 9 & 13.205 \\
\hline 1884. & 130.3 & $19.6 \mid$ & 11.3 & $\times 5.7:$ & 36.5 & - & 15.3 & 83.6 & 27.4 & 94.9 & 9 & 15.6509 \\
\hline 1885. & $14 \div .6$ & 19.9 & 8.7 & $91.5:$ & 27.8 & - & 15.7 & 80.93 & 31.5 & 100.9 & 9 & 15.1479 \\
\hline $1 \times 86$. & 144.3 & 15.2 : & 20.8 & 91.5 & 26.8 & - & 16.2 & 82.73 & 30.4 & 107.4 & 9 & 16.1806 \\
\hline 1887. & 200.1 & 8.3 & 23.5 & 95.6 & 30.1 & - & 16.6 & 88.83 & 37.9 & 121.1 & 9 & 18.0628 \\
\hline 1888. & $20 \div .8$ & 12.8 & 21.4 & 106.6 & 42.5 & - & 23.1 & 93.9 & 46.5 & 139.4 & 10 & 23.3817 \\
\hline 1889. & 210.5 & 7.1 & 29.7 & 139.0 & 40.6 & 100 & 23.8 & 105.8 & 46.6 & 170.8 & 10 & 28.1252 \\
\hline $1890 .$. & 223.3 & 11.4 & 29.7 & 115.13 & 34.0 & 150 & $2 \dot{i} .6$ & 101.05 & 51.6 & 151.7 & 9 & 38.3041 \\
\hline $1 \$ 91 \ldots$ & 241.5 & $7.3 \%$ & 26.9 & 86.92 & 28.0 & - & 25.1 & 85.05 & 54.3 & 142.0 & 8 & 25.559 \\
\hline $1 \times 92 .$. & 235.3 & $17.2:$ & 20.7 & $103.3:$ & 29.8 & - & 25.5 & 96.06 & 61.8 & 144.0 & 9 & 25.3312 \\
\hline $893 .$. & 227.9 & $19.8:$ & 21.7 & 105.73 & 36.6 & - & $|26.0|$ & 96.3 & 69.3 & 145.0 & 10 & 29.1526 \\
\hline
\end{tabular}




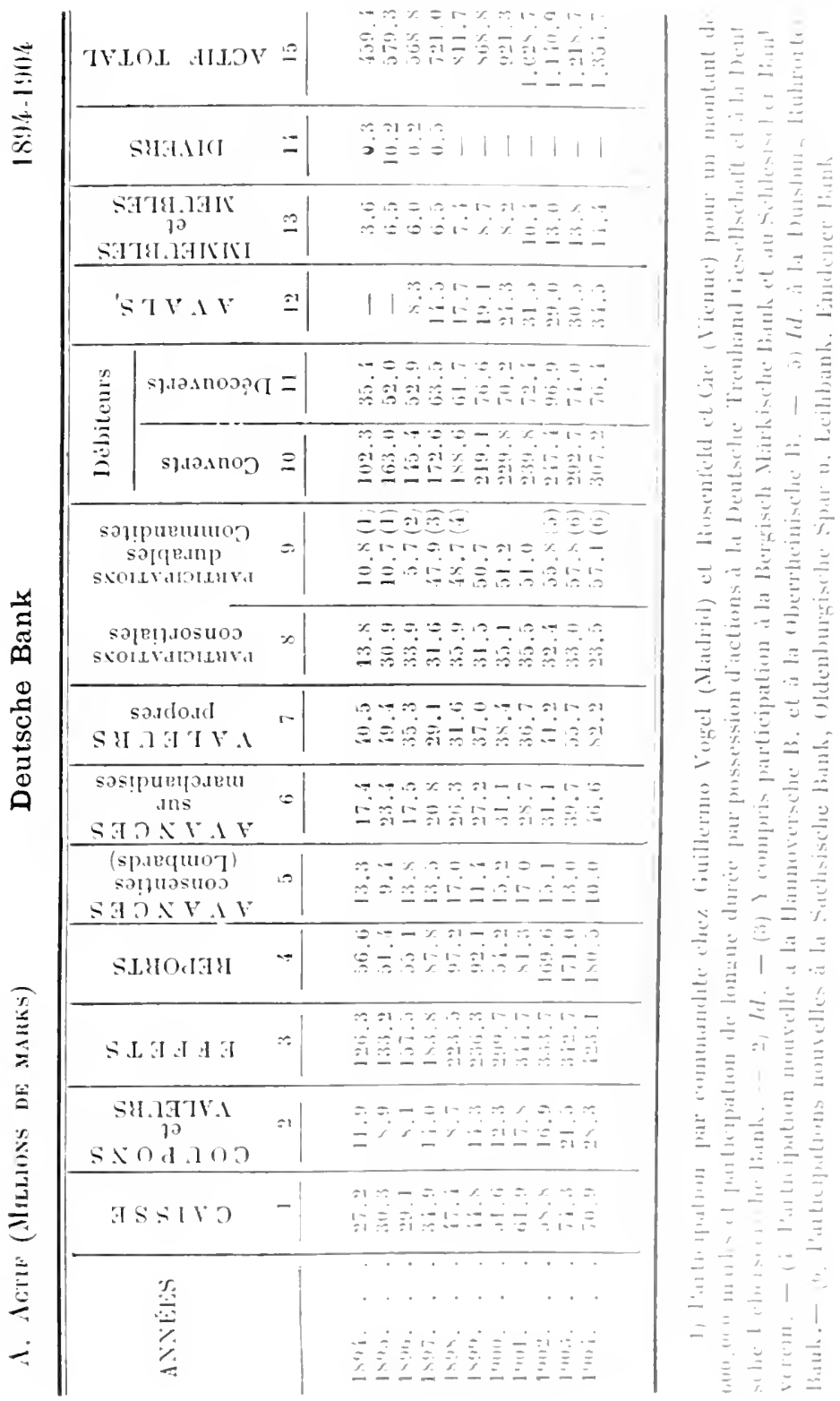




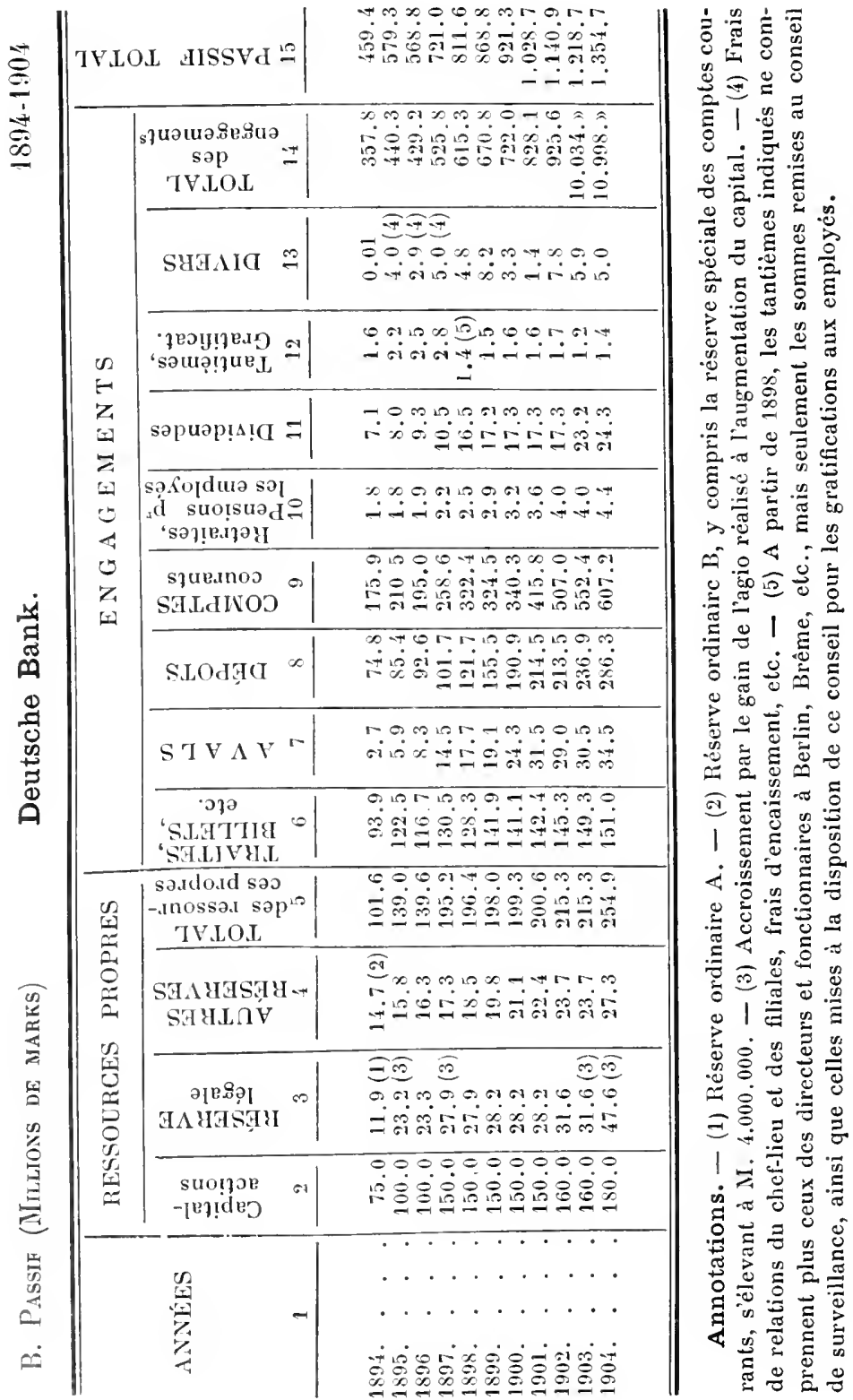




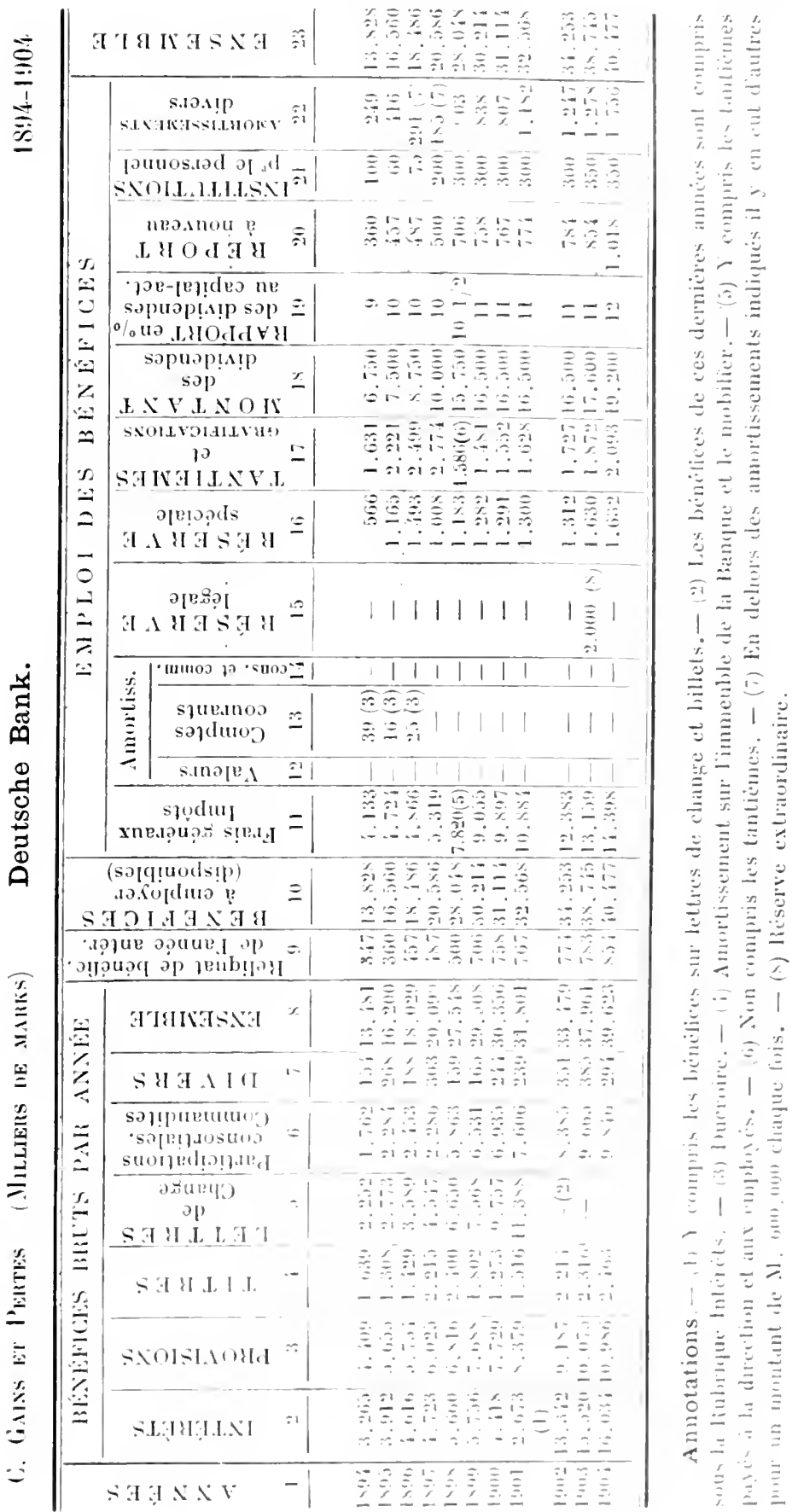


Deutsche Bank.

Bénéfices (millions de marks).

\begin{tabular}{|c|c|c|c|c|}
\hline Années & Bénéfice brut & Bénéfice net & gulières & participations \\
\hline 1870. & 0.4 & 0.3 & 0.3 & 0.1 \\
\hline 1871. & 1.4 & 1.2 & 0.9 & 0.5 \\
\hline $18 \% 2$. & 3.9 & 3.2 & 2. & 1.8 \\
\hline 1873. & 4.1 & 2.9 & 3.7 & 0.4 \\
\hline 1874. & 5. & $3 .: 3$ & 4.1 & 0.9 \\
\hline 1875. & 4.4 & 2.6 & 3.8 & 0.6 \\
\hline 1876. & 6.2 & 4.1 & 5.6 & 0.6 \\
\hline 1877. & 3.1 & 3.1 & 4.8 & 0.2 \\
\hline 1878. & 6. & 3.4 & 5.1 & 0.8 \\
\hline 1879. & 7.8 & 5.2 & 5.9 & 1.8 \\
\hline 1880 . & 8.6 & 6. & 6.9 & 1.6 \\
\hline 1881. & 9.2 & 7.3 & 7. & 2.1 \\
\hline 1882. & 9.1 & 6.6 & 6.9 & 2.1 \\
\hline 1883. & 8.7 & 6.8 & 7.3 & 1.2 \\
\hline $188^{\prime}$. & 10.3 & 7.4 & 7.9 & 2.3 \\
\hline 1885. & 10.3 & 7.4 & 7.5 & 2.7 \\
\hline 1886. & 10.3 & 7.6 & 6.9 & 3.1 \\
\hline 1887. & $10 .:$ & 7.5 & 7.4 & 2.6 \\
\hline 1888. & 11.5 & 8.3 & 8.5 & 2.6 \\
\hline 1889. & 14.8 & 11.2 & 10.8 & 3.5 \\
\hline 1890. & 15. & 11.2 & 11.6 & 2.9 \\
\hline 1891. & 13.7 & 9.4 & 10.7 & 2.3 \\
\hline 1892. & 12.2 & 8.1 & 9.3 & 2.5 \\
\hline 1893. & 12.2 & 8.1 & 10.7 & 1.1 \\
\hline $189 \dot{x}$. & 13.8 & 9.4 & 10 & 3.4 \\
\hline 1893. & 16.5 & 11.4 & 12.3 & 3.5 \\
\hline 1836. & 18.4 & 13.3 & 14 & 3.8 \\
\hline 1897. & 20.5 & 15.0 & 13.3 & 4.5 \\
\hline 1898. & 28.0 & 19.3 & 19.1 & 8.2 \\
\hline 1899 & 30.2 & 20.3 & 21.0 & 8.3 \\
\hline 1900. & 31.1 & 20.4 & $2 \mathcal{2} .0$ & 8.2 \\
\hline 1901. & 32.5 & 20.5 & 22.4 & 9.1 \\
\hline 1902. & 34.2 & 20.6 & 22.5 & 10.5 \\
\hline 1903. & 38.7 & 21.3 & 25.5 & 11.9 \\
\hline 1904 . & 40.4 & 26.0 & 27.0 & 12.3 \\
\hline
\end{tabular}

Produit des affaires de banque réProduit des titres et ulières 0.1 0.5 0.4 0.9 0.6 1.6 2.1 2.3 2.7 2.9 2.3 3.5 3.8 4.5 8.2 8.2 9.1 11.9 12.3 
II. - La formation du grompe de la boutsede Ibank.

l.a Deutsche Bank a sulecesiriment fondi los filinles (S suivantes: Brome (1871. Hamboure 1872. Lombres (1S73). Francfort $18 s 6$ par la repriec de liactif et lu passif du Frankfurter Bankverein. Munich 1sst. Leipzig. Dresde 1900 par absorption du Uroslnur Creulit Intalt et la reprise de la clientèle de la Luiprizer Bank: Nuremberg (mars 190\%).

Elle possède:

A Berlin et aux environs 27 Caisses de députs.

$\begin{array}{llrl}\text { A Dresde } & - & \dddot{3} & - \\ \text { A Hambourg } & - & 10 & - \\ \text { A Leipzig } & - & \ddot{3} & - \\ \text { A Wiesbaden } & - & 1 & -\end{array}$

Elle commandite :

Guillermo Vogel et Cie, à Madrid 1s9' 1 .

Rosenfeld et Cie. à Vienne 1s9; 1 .

G. E. Heydemann. à Bautzen. aree filiales à Lïban it Zittau 1903 .

La Deutsche Bank a fondé ou participé à la fondation des societés filiales suivantes:

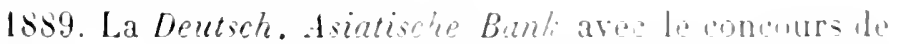
la Bank fïr Handel und Industrie, le la Dre-dner liant. de la Discontogesellschaft et le l'A. Seharbhau-en-illel Bankiv.

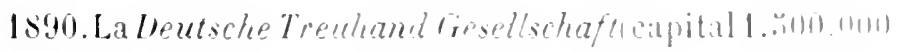
marks.

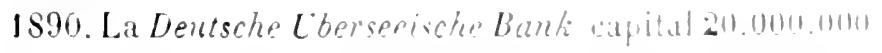

(1) Col. a de l'acrif. 
Filiales à Santiago, Valparaiso, Antofagasta, Concepcion, Iquique, Buenos-Ayres, Valdivia, Bahia-Blanca, Mexico, Lima et Barcelone.

1895. La Banque d'entreprises électriques (Zurich), capital 33.000.000 de francs.

1896. La Banque des chemins de fer orientaux (Zurich), capital 50.000 .000 dont 15.000 .000 de francs versés.

1899. La Banca Commerciale Italiona (Mailand), capital 80.000 .000 de lires, avec le concours d'autres banques suisses, allemandes et autrichiennes.

23 filiales en Italie.

1904. La Deutsche Ostafrikanische Bank (capital: 2.000.000 de marks), avec le concours des autres grandes banques allemandes.

Constitution du groupe de la Deutsche Bank : la caractéristique de la formation do ce groupe réside dans le nombre extrèmement peu élevé des absorptions pleines et entières de banques locales réalisées par la Deutsche Bank elle-même : elle a généralement procédé par acquisition d'un nombre d'actions suffisant pour assurer conformément à ses vues, la direction de l'établissement secon. daire.

\section{$1^{\circ}$ Absorptions de la Deutsche Bank.}

1886. Frankfurter Bankverein.

1901. Menz, Blochmann et Cie, Dresde.

1905. Chr. Schmidt, à Ilambourg.

$2^{\circ}$ Participations par possession d'actions.

a) 1897. La Deutsche Bank augmente son capital de 50 


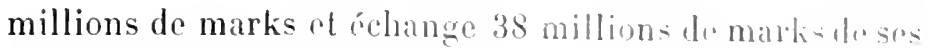
nouvelles actions contre fl millions do mark d'allions de la Bergisch Mærkische Bank d'lillurfuhl.

La liergisch Mirkische liank fondée en 1871 au rapital te 13.500.000 marks - ramené en 1881 i 10.800 .1160 marks, - a depuis 1897 ojéré les augmentations de capital suivantes:

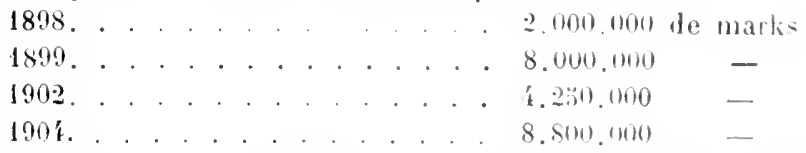

Elle représente en lin 190's un capilal de 60.01119).1110) de marks plus 15.400.000 marks de ré:erres.

la Rergisch . Viarkische Bank possède:

13 tiliales.

2 caisses de dépòts.

3 commandites.

Elle a successivement absorbé les maisons suivantes:

1895. R. Jacobi Cologne).

- J. H. Brinck et Cie (Elberfeld).

- Salomon Philipp (Ruhrort).

1898. La Remscheider Bank (Kuhrort: au capital de 2.000 .000 de marks qui est deventre sum celle raison so. ciale une filiale de la Berg. Mark. li.

1901. A. et C. Lohmann (irefeld.

- Goldschmidt et Cie (Bonn).

- Beckeratl et Heilmann Cirefeld.

- A. Suermondt et Cie lix la-(hapelle).

1902. La Barmer Handelsbank.

190;. Le Padersteinscher Bankverein rapital : 8000.1110 de marks), l'adprorn.

- Lazard Brach et Cie, i saint-Jean-silarbuck.

- La Trierer Bank capital 2. m14t.100 de mitris.

- A. Molenaar et Cie (capital 1.2611.1149 markis). i Cireleh.

La Deutsche Bank et la liersisch Mardische liank out phames deux de leurs directeurs décenés au consil d'administation de l'autre banque. 
b) 1897. Les 12 millions de marks des nouvelles actions de la Deutsche Bank qui lui restaient après l'échange opéré avec la Bergisch Märkische Bank étaient destinés à acquérir - après conversion - les 27 millions de marks d'actions représentant le capital du Schlesischer Bankverein de Breslau.

Le Schlesischer Bankverein fondé en 18:16 au capital de 6.900 .000 marlis a successivement augmenté son capital :

$\begin{array}{lccc}1870 . & \text { capital } & 15.000 .000 & \text { de marks. } \\ 1871 . & \prime & 18.000 .000 & " \\ 1876 . & " & 20.000 .000 & " \\ 1889 . & " & 22.500 .000 & " \\ 1896 . & " & 27.000 .000 & " \\ 1904 . & " & 30.000 .000 & " \\ & \text { réserves } & 9.000 .000 & "\end{array}$

Le Schlesischer Bankverein possède:

9 filiales.

3 commandites.

Il a absorbé :

1905. La succursale d'Hirschberg de la maison Abraham

\section{Schlesinger.}

Il délègue un directeur au conseil d'administration de la Deutsche Bank.

La Deutsche Bank dé!ègue deux directeurs au conseil du Schlesischer Bankverein.

c)1897. La Deutsche Bankacquiert pour 4.000 .500 marks d'actions de la Hannoversche Bank; en 1899 elle resserre encore ces liens par l'acquisition de 2.499.000 marks de nouvelles actions de la Hann. B.

Le capital primitif de la Hannoversche B. 16.260.000 marks (1856), ramené à 12 millions en 1870, atteint en 1904, 22.500.000 marks ; les réserves sont de 3.300.000 marks en chiffres ronds. Elle possède 6 filiales.

La Deutsche Bank délègue un de ses directeurs au conseil de la Hannoversche Bank. 
d) 1898. (Yuand en 1897, l'Oberrheinische Bank portasun

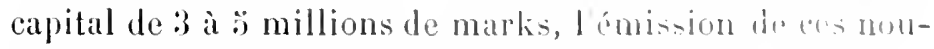
velles actions fut garantie par la Deutsche Bank. Mais la Deatsche Bank n’a jamais donné le chilfre ractules autions qu'elle avait aequises; le chilfre porti anx "valeute fropres n'augmente en 1898 que de deux millions sur l'anmón précédente; d’autre part, cette mème année la beutsho Bank diminue sa participation it la Deutsche Clyerserischu Bank.

L'Oberrheinische liank qui fusionna en 190' avec la Rheinische Credit Bank représentait un capital de 20.000 .000 de marks.

Elle possédait : 9 tiliales,

$$
\begin{aligned}
& 1 \text { caisse de dépòts, } \\
& 2 \text { commandiles, }
\end{aligned}
$$

et avait successivement absorbè les maisons:

1883. Kœster et Cie, a Mannheim.

- C. Schwarzmann, à Strasbourg.

- C. Mez, à Frihourg.

1903. Ed. Koelle, à Karlsruhe.

- R. Nicolaï et Cie, à Baden-haden.

- J. S. Meyer, à Baden-Baden.

Elle était intéressée à la Süddeutsche Bank de Yannheim.

e) En 1902, la Deutsche Bank porte son capital de 1.40 it 160 millions pour reprendre la suite des allatires de lat Duisburg Rurhorter Bankde Duishenre, calpital 12.00(1)0.0100 de marks), fondée en 187 't all capital de 2 millions.

La Duisburg Ruhrorter Bank posiédait deux tiliales dont une a Dússeldorf sous la raison sociale, Niederrheinische Bank.

f) En 1902, la Deubcho Bank Belange puatre millinns

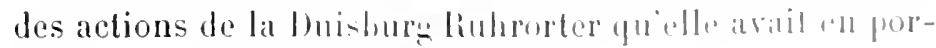
tefeuille contre $: 0.001 .000$ d'action- d. l'Essener Credit

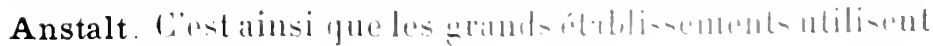


parfois les actions de banques secondaires qu'elles possèdent pour se créer de nouvelles relations. La Deutsche Bank possédait déjà en propre 200.000 marks d'actions de l'Essener Credit Anstalt.

L'Essener Credit Anstalt fondé en 1872 au capital de 9.000.000 de marks l'a successivement augmenté comme suit:

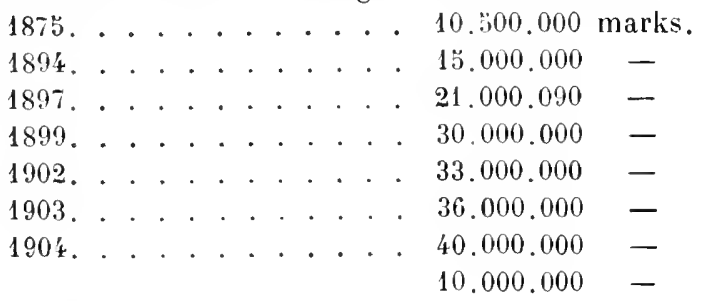

Il possède: 10 filiales ou agences,

$$
2 \text { commandites, }
$$

et a successivement absorbé les maisons :

1895. L. Hirschland, à Essen.

1902. La Credit Bank, à Recklinghausen.

1903. S. Hanf, à Witten.

Il est intéressé, comme nous l'avons vu, à la Duisburg-Ruhrorter Bank.

Et depuis 1904 au Westfælischer Bankverein, de Münster (1 filiale), capital 7.000.000 de marks dontil possède 1.000.000 de marks d'actions.

g) En 1904, la Deulsche Bank reprend la suite des affaires de la Siegener Bank für Handel und Gewerbe (capital 4.000 .000 de marks).

h) En 1904 elle acquiert, toujours par achat ou échange d'actions, des participations dans les banques suivantes:

Sæchsische Bank, à Dresde(capital 30.000.000 de marks) 8 filiales.

Essener Bankverein, ì Essen (capital 7.5000.000 marks) qui absorba en 1899 la maison Rebling et Rehn.

3 filiales. 


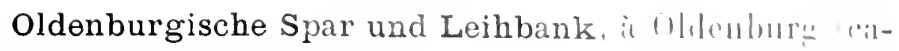
pital 4.000.000 de markis.

6 liliales.

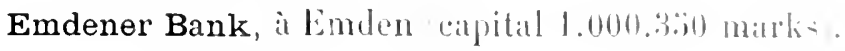

1 filiale.

Privatbank zu Gotha (apilal 7.200.000 marks).

3 filiales.

30 Entin la Deutsche Bink se trouve alliée contractuellement (communautés dinterrits proprement dite) illece:

$1^{\circ}$ La Mecklemburger Hypoteken und Wechsel Bank de Schwerin, depuis 1887.

Celte banque fondée en 1871 au capital de 9.0100.000 de marlis possède: 1 liliale.

60 agences.

Elle participe par possession d'aclions a la Mecklemburgische Sparbank (capital 3.000.000 de marks. 7.; asences.

La lJeutsche Bank a un délézué au conseil de la Merklembnrmel Bank; celle-ci, un délégué au conseil de la Deutsche liank.

$2^{\circ}$ La Rheinische Credit Bank de Minnheim, depuis 190 '.

Cette banque fondée en tri: au capital de 2.010. (1106) de llatlers a successivement porté son eapilal:
at 15.000 .000 marks en 185.9 .

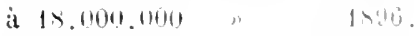
a 20.100 .000 " " $1 \times 9 \%$.
à 30.000 .1000 " 1 1>95.
a $\$ 0.000 .800$ n 149.
à 46.000 .8000 " " 19111 .
à $70.000 .800 n$ n 18018 .

plus 10.000.11n0 réserves.

Elle possède 11 liliales,

2 agences.

3 commandites. 
Elle a absorbé les maisons suivantes :

1897. G. Müller et Cie, à Carlsruhe.

- F. Funck, à Baden-Baden .

1898. Kaiserlauterer Bank.

1899. Ostenauer Creditbank, à Offenbourg.

- Kauffmann Engelhorn et Cie, à Strasbourg.

- Ed. Strohmeyer, à Baden-Baden.

- Lahrer Creditbank, liarl Bader et Cie.

1901. Mannheimer Bank (capital 6.000.000 de marks).

1904. L'Oberrheinische Bank (capital 20.000.000 de marks).

- Kapperer et Cie, à Fribourg.

- Credit und Depositen Bank, à Zweibrücken (capitalactions 4.000 .000 de marks).

Elle est intéressée par possession d'actions :

1901. à la nouvelle Mannheimer Bank (capital 1.000.000 m.).

1904. à la Süddeutsche Bank de Mannheim (capital 10.000.000 de marks) dont elle possède pour 2.000 .000 de marks d'actions.

La Deutsche Bank et la Rheinische Gredit Bank délèguent chacune deux directeurs dans le conseil d'administration de l'une et de l'autre banque.

Nous avons vu que la tentative d'absorption (1904) de la Berliner Bank par la Deutsche Bank qui avait à cette intention porté son capital à 180.000 .000 de marks, n'a pas abouti.

Le tableau suivant des dividendes distribués par les principales banques affiliées au groupe de la Deutsche Bank montrera les bénéfices que cette dernière a pu retirer de ses participations:

\begin{tabular}{|c|c|c|c|c|c|c|c|c|}
\hline \multirow{2}{*}{\multicolumn{9}{|c|}{$\begin{array}{l}\text { Pergis ch } \\
\text { Märische }\end{array}$}} \\
\hline & & & & & & & & \\
\hline Bank. . & $71 / 2$ & $71 / 2$ & 8 & $81 / 2$ & $81 / 2$ & $81 / 2$ & 8 & 8 \\
\hline Schlesischer & & & & & & & & \\
\hline $\begin{array}{l}\text { Bankve- } \\
\text { rein.. }\end{array}$ & 7 & 7 & 7 & $71 / 2$ & 7 & $61 / 2$ & $61 / 2$ & 7 \\
\hline $\begin{array}{l}\text { Hannovers- } \\
\text { che Bank. }\end{array}$ & $54 / 5$ & 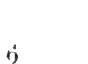 & 6 & 6 & 6 & 4 & 6 & \\
\hline Crte Dank. & & & 0 & & & $\mathbf{x}$ & 0 & $b$ \\
\hline
\end{tabular}


Oberrhein i s c h e liank. . DuisburgRuhrorter B. . EssenerCre. dit Anstalt ... siegener Bank. . Sächsische Bank. . Es sener Bankverein... - $\quad$ - - -

Mecklemburger Hyp. B. R hein ische C r e d i t Bank. . - - - - -

III. - Les partieipations industrielles et commeroiales de la Deutsche Bank. - Les affinires comsortiales.

La Deutsche Bank note sous le titre "Konsortial Kont" " les entreprises qu'elle a créces ou a la fondation de-ffunder

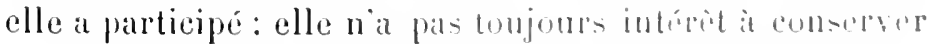
par devers elle la totalité on la matjorité des atelions. wlle les garde seulement jusqu'it ce quelles solunt. puor aliu-i dire, mùres pour l'émission.

sous ce titre rentrent épalement les participalion- prises par la banque dans les emprunts communand it lis emprunts d'litats.

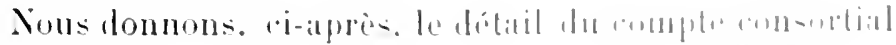


des deux exercices 1903-1904. Rappelons auparavant les principales participations de la Deutsche Bank depuis sa fondation jusqu'en 1902 :

$1^{\circ}$ Entreprises de transport et de navigation, au nombre de 27, parmi lesquelles:

Tramways berlinois.

Hamburg Amerilia (Packetfahrt gesellschaft). . .

Norddeutscher Lloyd.

Gesellsch. für elektrische Hoch und Untergrundbahnen.

2 Banques, banques hypothécaires et Compagnies d'assurances: au nombre de 92.

$3^{\circ}$ Entreprises industrielles:

a) Brasseries au nombre de 25.

b) Aciéries et Charbonnages (\$4) dont voici les plus importants :

Actiengesellschaft Schalker Gruben und Hüttenverein.

Bergwerksgesellschaft Centrum.

Gewerkschaft Mont-Cenis.

Gewerkschaft Hohenzollern.

Rombacher llüttenwerke.

Rheinische Stahlwerke.

c) Industries textiles (2't).

d) Industries chimiques et raffineries (17).

c) Industries électriques (14), entre autres les deux sociétés qui ont donné les meilleurs résultats financiers :

Siemens et llalske;

Allgemeine Elektricitätsgesellschaft.

f) Constructions de machines et industries métallurgiques (32);

Frederich Krupp (Essen). 
Lokomotivfabrik kians ol lio.

g) Briqueteries $(\ddot{i})$.

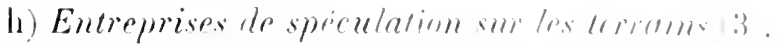

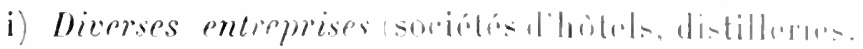

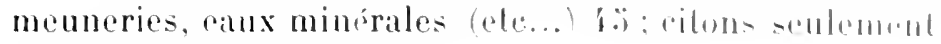
Le Nobol Dymamit-Trust.

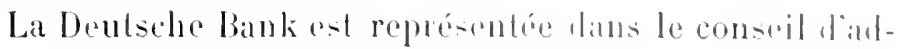
ministration de 28 de cessocialés:

9 Mines el Charbonnanes.

a Constructions do machines.

1:3 Entreprises électripues.

2 Entreprises chiminues.

$4^{0}$ Eulin, la part que la Dentsche bank a prise tans l'émission de tous les fomels deEtat allemands on chantrgrers ainsi que dans los emprumts des grandes villus at considérable.

Elle ne comple pas moins, toujours jusquen 19t12. 10: participations amx emprouts d'ktals allemands. 13

\begin{tabular}{|c|c|c|}
\hline & $"$ & de provintes allem. \\
\hline & ) & de villes n \\
\hline & $n$ & Elals citrangers \\
\hline & $"$ & villes rillongeres \\
\hline
\end{tabular}

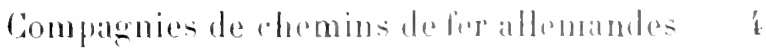

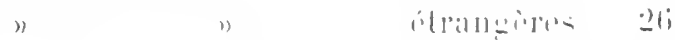

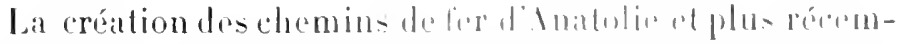

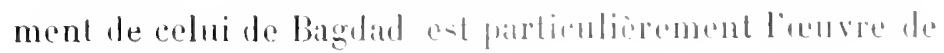
la Deulsche Bank.

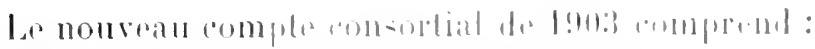

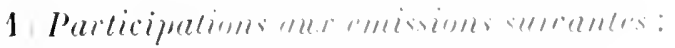

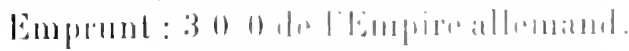

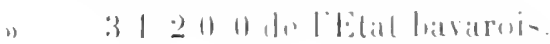


Emprunt: $31 / 20 / 0$ du Wurtemberg.

$31 / 20 / 0$ du Grand ducal Landes Credit Anstalt de Gotha.

$41 / 30 / 0$ de l'Etat autrichien (conversion).

$41 / 200$ obligations du Trésor mexicaines.

$31,200 "$ " " finlandaises.

$31 / 200$ des villes de Dresde, Düsseldorf, Elbing, Essen, Harburg, Herne, Mülheim, Munich, Posen et Rostock.

40,0 de la ville d'EIberfeld.

$33,40,0$ (obligations foncières) de la ville de Dresde, de la ville de Leipzig (conduit par la Leipziger Filiale).

40,0 de la ville de Drammen (conduit par la Hamburger Filiale).

4120,0 de la ville de Warschau.

400 (obligations foncières) de la Necklemburg-Hypoteken und Wechsel Bank et du Preussisch. Boden Credit Anstalt.

$41 / 200$ (obligations) de la Magdeburger Bau und Credit Bank.

$4120 / 0$ (obligations) du Stockolms Interknings Garanti Aktiebolag.

$31 / 200$ (obligations) de l'llaberstadt Blankenburgen Eisenbahn Gesellschaft et de la Böhmischer Nordbahn Gesellschaft.

o) $0 / 0$ (actions de priorité) de l'Osterreischer Nordwestbahn.

$40 / 0$ Prior Lien Bonds du Northen Pacific Railway.

4 0,0 First Mortgage Bonds du Central Pacific Railway. 


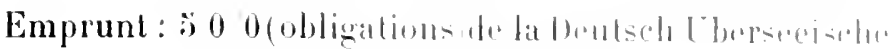
Elektricitiatsesentlschalt.

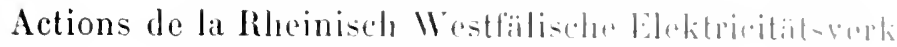
A. G.

Actions de l'Lnionshrauerei Schiilein et Cis $\Lambda$. C. (Bayerische Filial").

Emprunt 400 du Schalliep Guben umbl Itittenserein, du Rheinische Anthracit-liohlenwertin, de la Bremer Tauwerfabrik Bremer filialo. de la Vereinsbierbrauerei de Leipeige ot da la Leipziger Nalzfabrik Leipziger filialo.

)

$41 / 200$ du Tratikakticbotaged de liringesberg-( )xelösund.

Actions de la Banque d'entreprises électriques, ì Zurirh. 20 Emessions conduites par la Deutschr Bunli sulo:

Emprunt 3120,0 de la ville de Cöthen.

"de la ville de Kiel.

" $31 / 2$ et 40 0 obligations foncieres de lillypotekenbank de llambonres. de l'Ilannoversche Bodeneredilhank at du Wistentsche Boden Credit Anstill.

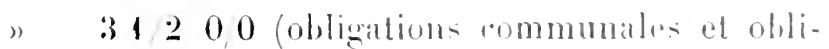
gations foncieres de la llusicche landes Iypotekeubank.

Actions de La Bergmann Elektricitiat-- Verker A. 1i, du Deutseher Wagron-Leihanstalt ad de la Roivehelt Metallschrauben A. G.

$3^{\circ}$ Fondations.

Schöneberg. West 1. (i. Riir Gerund-turkisarwertung.

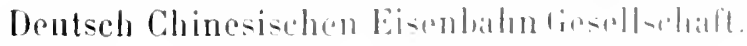

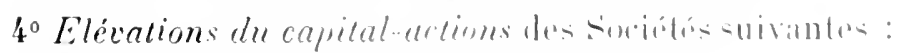

Schlesischer Boden Credil Mktien tiank. 
Hypotekenbank, ì Hambourg.

Essener Cireditanstalt.

Deutsche Dampschiffahrts Gesellschaft IIansa.

Banca Commerciale Italiana.

Le nouveau compte consortial de 1904 se décompose ainsi :

$1{ }^{\circ}$ Participation aux émissions.

Emprunt $30 / 0$ de l'Etat bavarois (Münchener Filiale).

" $31 / 20 / 0$ du Duché de Bade.

" $30 / 0$ du Duché de Hesse.

" $31,20 / 0$ de la ville du Lubeck.

" $31 / 20 / 0$ du district de la Haute-Bavière (Münchener Filiale).

" $50 / 0$ de la République de Cuba .

" $4120 / 0$ bons du Trésor mexicains.

" $31 / 20 / 0$ des fivilles de Barmen, Duisbourg, Düsseldorf, Ilamm et Mannheim.

" $\quad 400$ de la ville de Nuremberg.

" $\quad 33 / 4,0,0$ (obligations foncières) de la ville de Dresle.

$3120 / 0$ de la ville de Francfort (Frankfurter Filiale $)$.

$40 / 0$ (obligations foncières) de la Preussisch Hypoteken Aktien Bank.

" 400 (obligations foncières) de la Preussisch Boden Credit Aktein Bank.

4 $0 / 0$ (obligations foncières) de la Mecklemburg. Hypoteken und Wechsel Bank.

4 0 0 I. Refunding Mortgage Bonds du Central Pacific Railway Company. 
Emprunt: 400 Kotes du Xew-York, Xew Hatren ut Hartford Riallia! Cimplany.

)

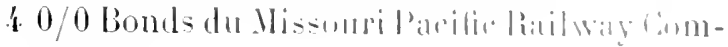
pany.

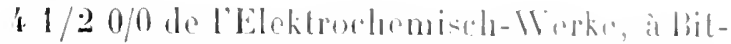

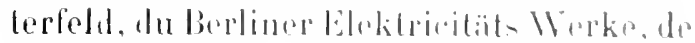

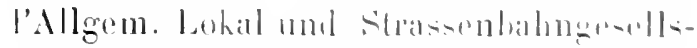
chaft, de l'Ekhilrisch. Licht mol kiraftanlagen $A$. G .

\$0 0 bobligalions of actions du seblesich. Elektricitits und hats. A. G.

41200 (obligations) de la lentich l'bersecische Eloktricititstacellshbilt.

$" \quad 1200$ de la maison 1. Bursiz.

" 1200 de la Falkensteine hitrdinenurobed und Bleicherei et de la maison li. Teichert (I)reselner Filiale).

$" \quad 600$ de la Weser 1. (i. Lliremer Filiale).

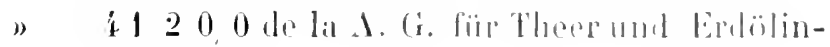
dustrie.

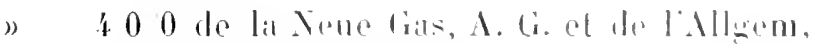
Gias. 1. li.

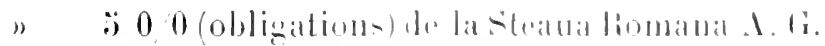
fïr Petrolimm Industrie.

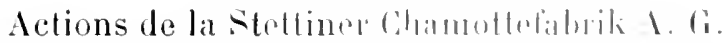

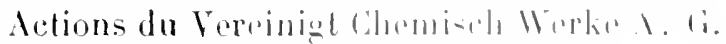

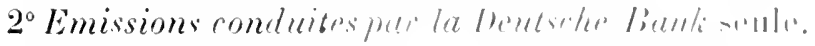

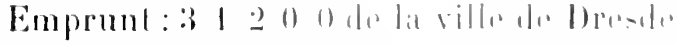

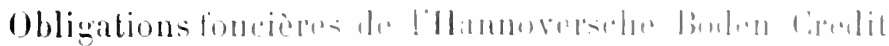

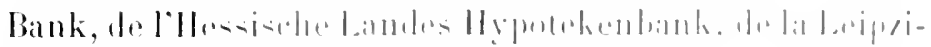

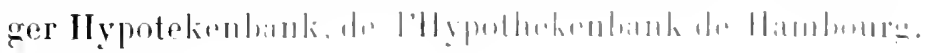


Emprunt : $40 / 0$ (obligations) du Creditverein à Copenhague.

$3^{\circ}$ Conversions.

Emprunt de la ville de Rome.

Obligations foncières de la Schwedische Reichs-Hypotekenbank.

$4^{\circ}$ Elévations du capital-actions des Sociétés suivantes: Deutsch Asiatische Bank.

Deutsch Ueberseeische Elektricitäts Gesellschaft. "Königsborn " A. K. lür Bergbau, Salinen und Soolbadbetrieb.

Nïhmaschinenfabrik und Eisengiesserei A. G.

Farbenfabrik Fried. Bayer et Cie.

" Meister Lucius et Brüning.

Harpener Bergbau A. G.

Bergisch Märkische Industrie Gesellschaft.

Georgs-Marien-Bergwerk und Hüttenverein.

Schubert et Salzer A. G. (Dresdner Filiale).

G. Secbeck A. G. (Bremer Filiale). 
I.A DEETSCHE B.INK

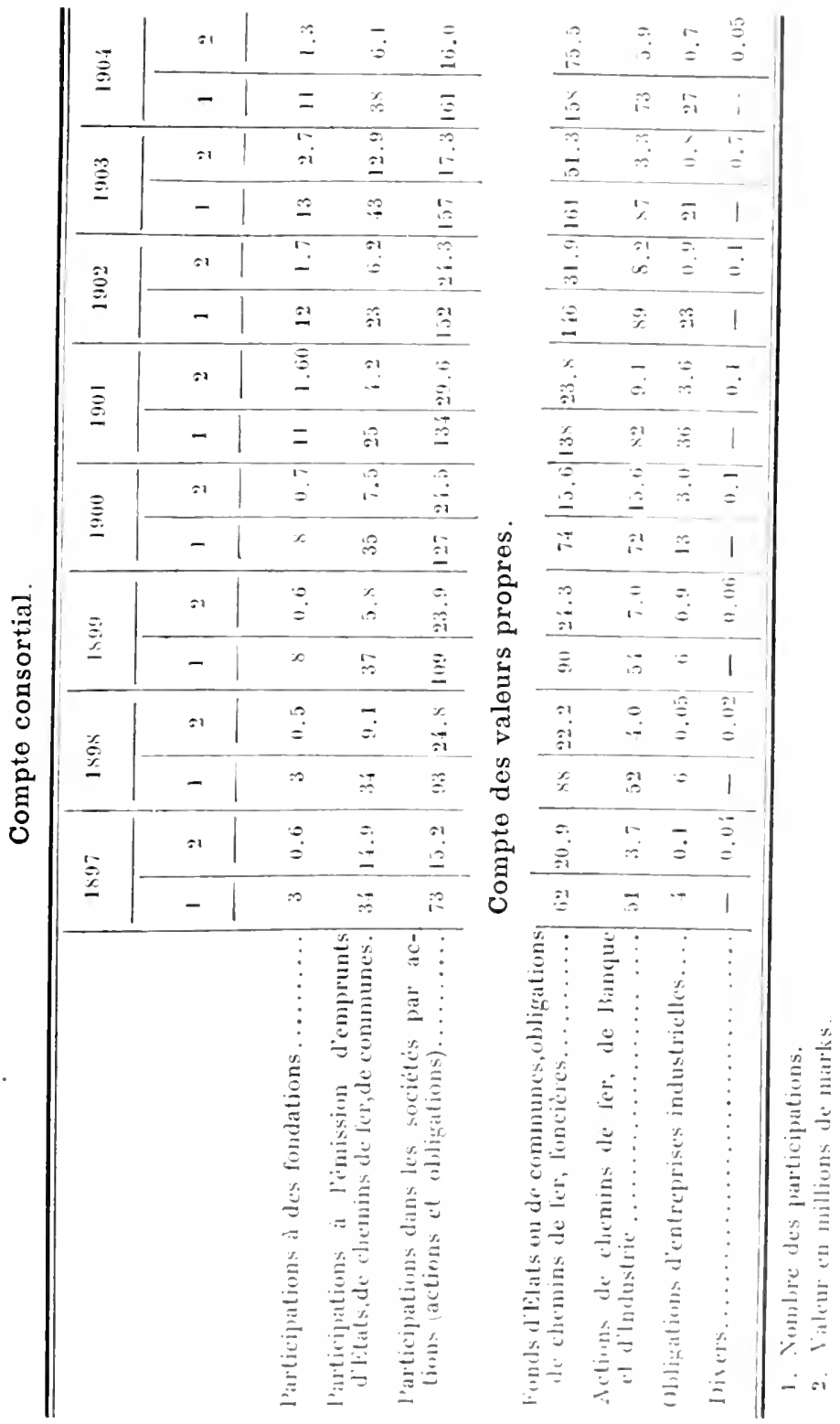




\section{CHAPITRE IV}

\section{LA DRESDNER BANK ET LA. SCHAAFFHAUSENS'CHER BANKVEREIN.}

1. - Le développenent propre de la Dresdner Bank et de l'A. Schaalfhausens'cher Bankverein.

A. - La Dresdner Bank fut fondée en 1872 à Dresde par un consortium de banques, - Berliner Handelsgesellschaft, Leipziger Credit Anstalt, Deutsche Vereinsbank, Anglo Deutsche Bank - qui ne prévoyaient point la concurrence victorieuse que leur jeune création allait bientòt leur faire.

Le capital originaire de la Dresdner Bank (22 millions de marks dont 400 0 0 versés) a subi les modifications suivantes:

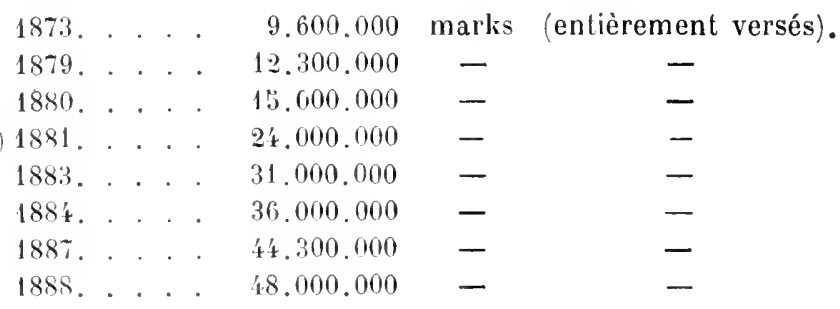

(1) A l'occasion de la création de la filiale berlinoise. 


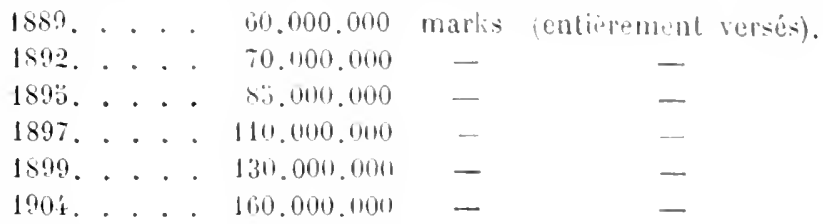

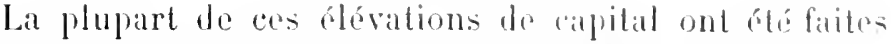
pour absorber des bintues secondalires Voirs II.

Le siege social de ha hanciue est tonjours a lmesde, mais son principal établissmont est à borlin.

Lexamen des bibans de la lpestner bank nous permet de constater:

10 Laictivité de la banque a toujours b́tín progeressiunt

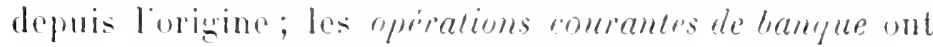
suivi un développement très régulier arece un líger recul de trois années lors de chacune des crises 1591 et 1900 , saceroissant à mesure que lis relations de la bandue sétendaiont davantage.

20 Les bénélices provenant des opératime finameiripes témoignent an contratire d’une grande irrégulatrití: lat

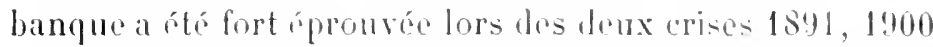

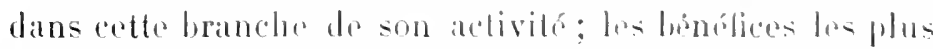

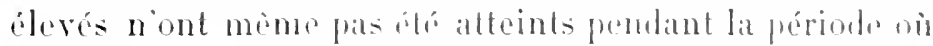

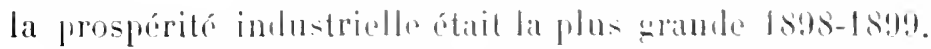

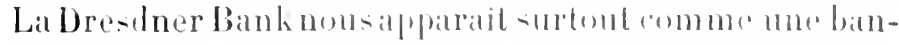

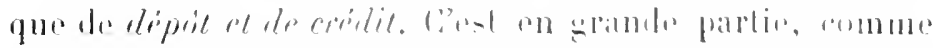

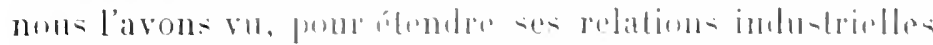

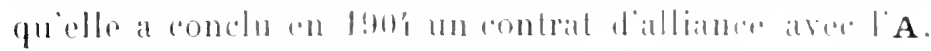
Schaaffhausens'cher Bankverein de Cologne

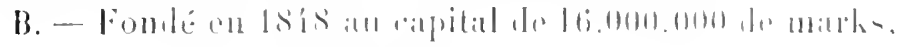

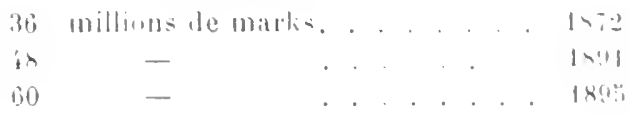


75 millions de marks. . . . . . . 1897

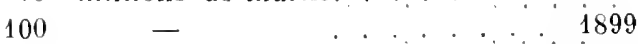

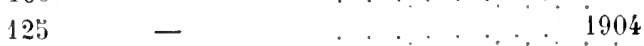

l'A. Schaaffhausens'cher Bankverein montre, jusqu'en 1900, un accroissement des principaux chapitres de son bilan; les opérations de banque pure se sont accrues régulièrement mais dans une moins forte proportion que le chapitre titres et participations, ses opérations comportant en majorité des engagements à longue échéance, il a subi des pertes assez fortes lors de la crise 1900-1901; c'est gràce aux bénéfices plus considérables réalisés sur les opérations régulières de banque en 1903, 1904, qu'il a pu augmenter son dividende ces deux années.

Cette augmentation d'une part, et de l'autre celle dı chapitre titres et participations à la Dresdner Bank, montrent l'influence que peuvent exercer l'un sur l'autre deux établissements, l'un ayant son siège dans une région industrielle et très directement intéressé dans un grand nombre d'entreprises, l'autre ayant au contraire son activité plutôt dirigée vers les opérations de dépôt et de crédit. Ils se complètent et se fortifient; les résultats de l'exercice 1904, encore qu'ils ne permettent pas de porter un jugement définitif, montrent les premiers bons effets de cette entente. 
Millions de Marks Dresdner Bank.

1572-15:1?

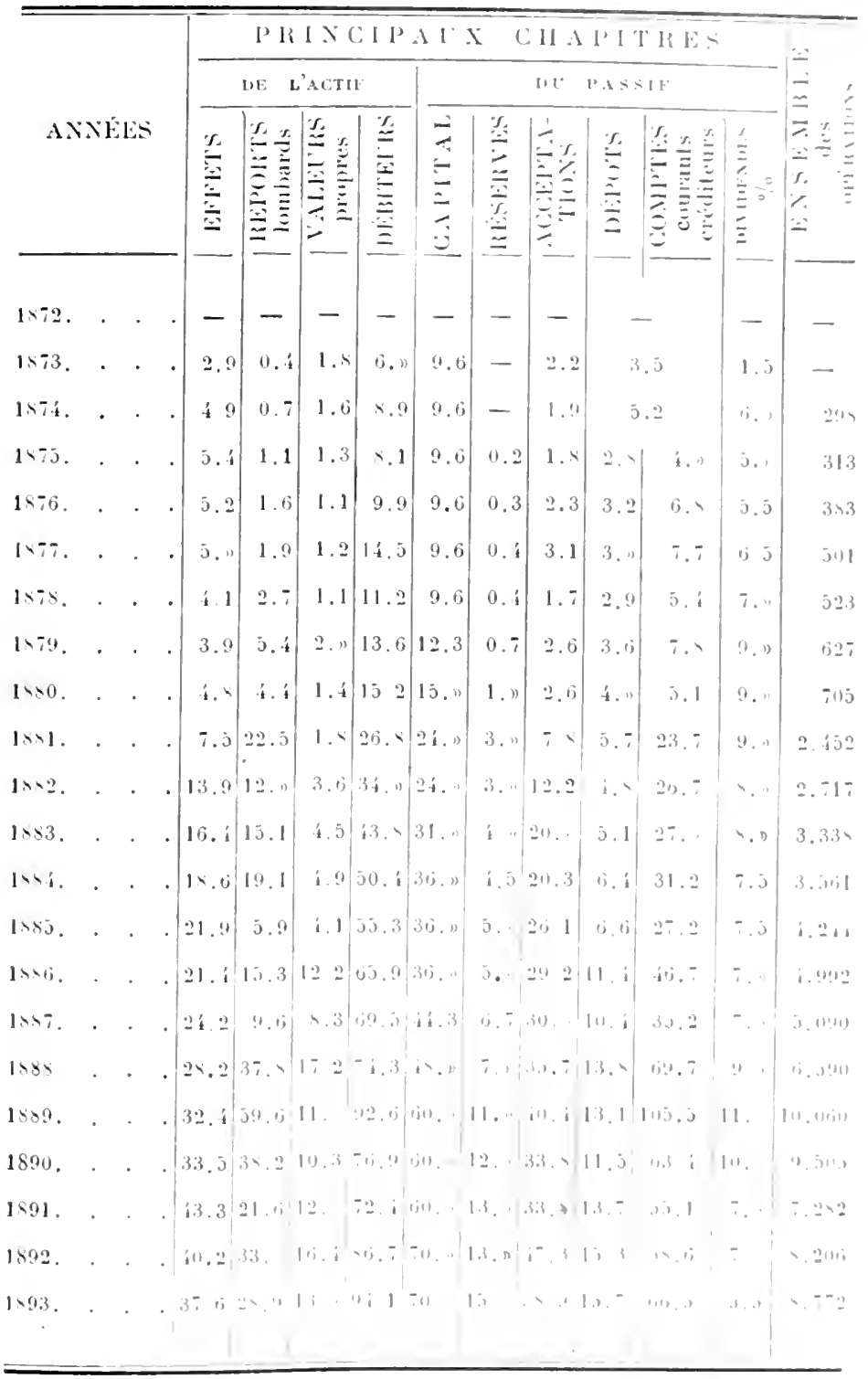


DELXIEME PARTIF. - CHAPITRE IV

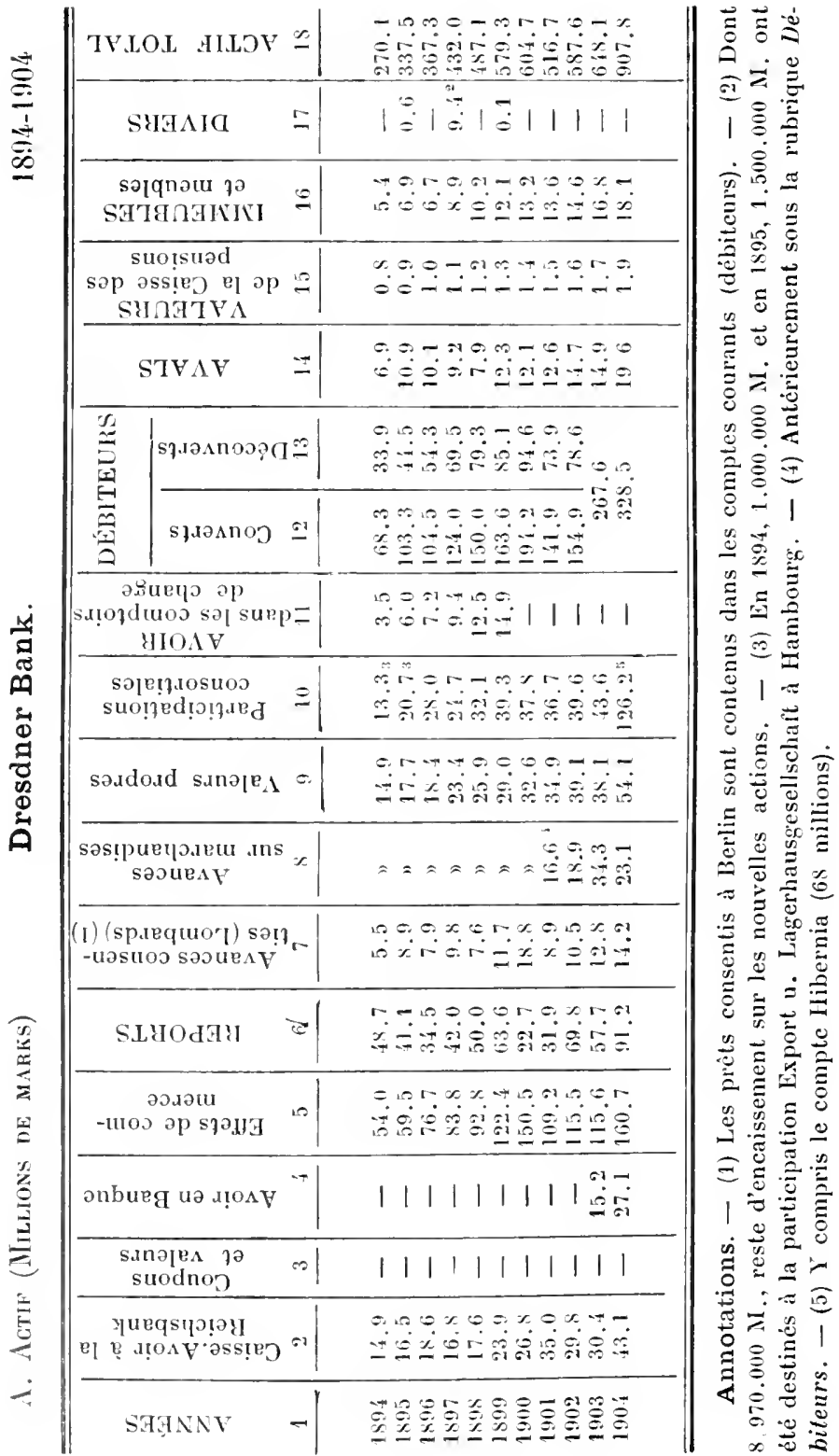




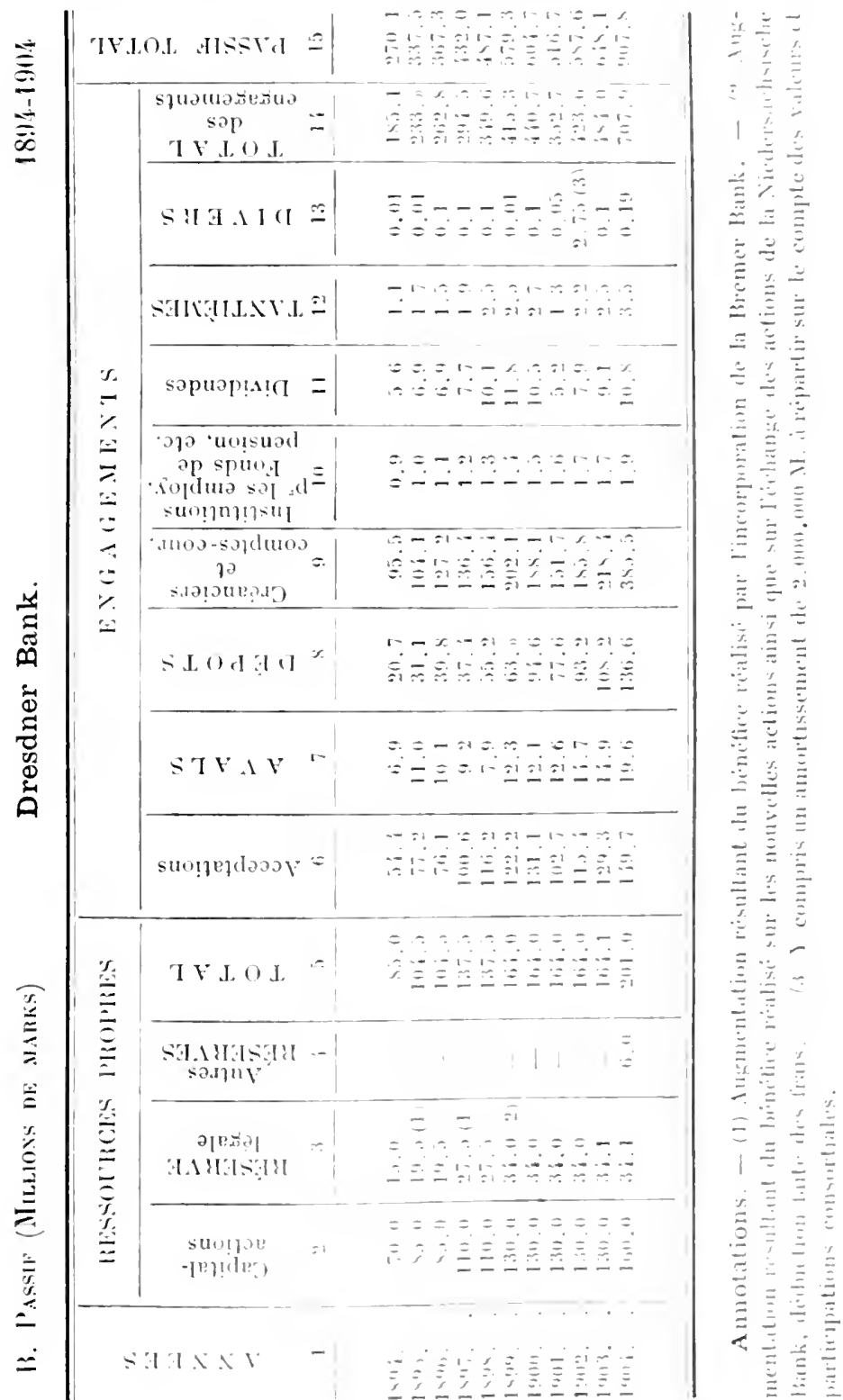




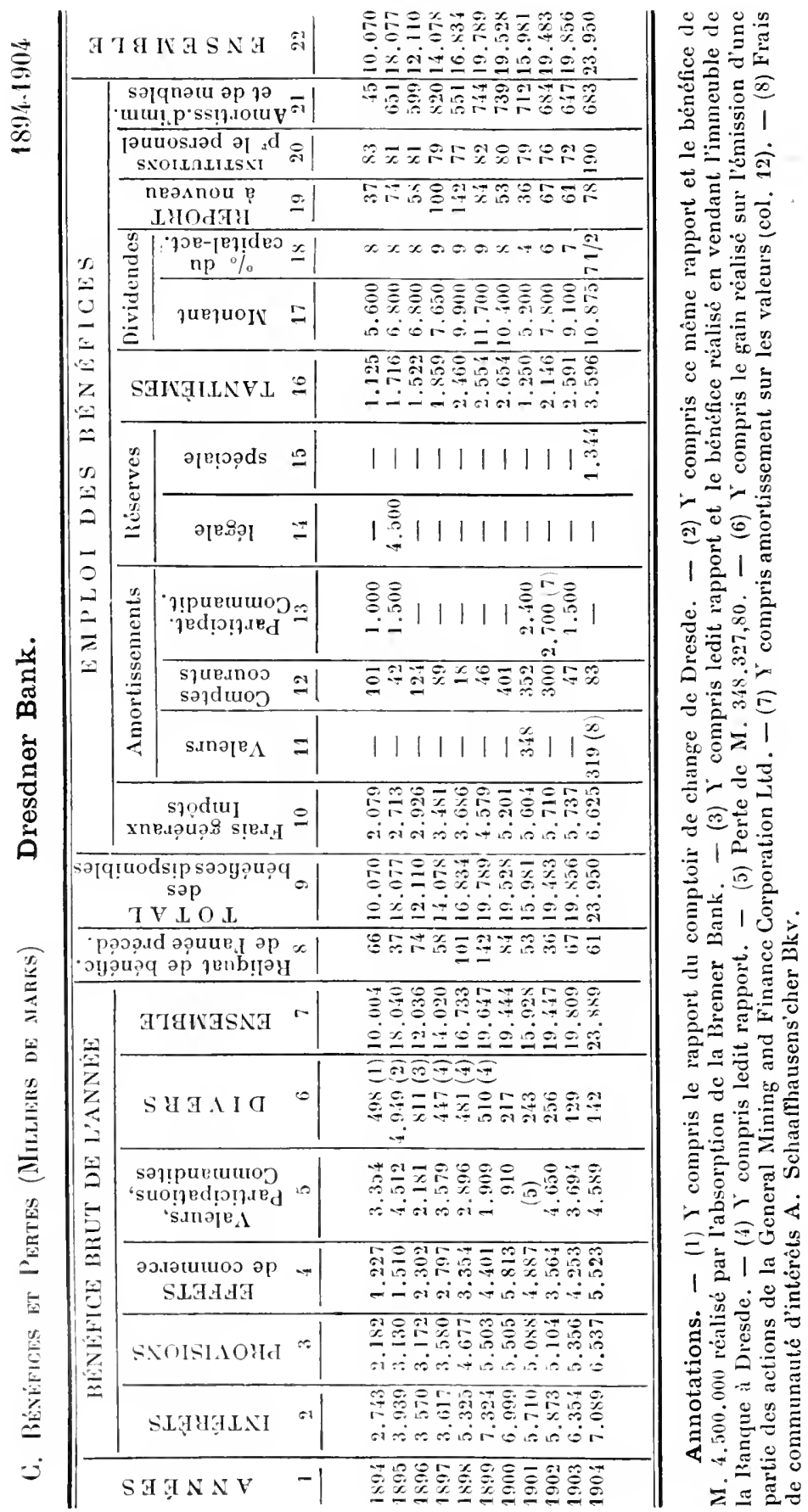




\section{Dresture Banti.}

Bénéfices (millions de marks).

\begin{tabular}{|c|c|c|c|c|}
\hline Annèes & Bénéfice brut & Benefice net & $\begin{array}{l}\text { banque ré- } \\
\text { gulieres }\end{array}$ & $\begin{array}{c}\text { titres el } \\
\text { parlicipations }\end{array}$ \\
\hline $18 \% 3$ & 0.6 & 11.3 & $11 . ;$ & 0.1 \\
\hline $18 \% \mathrm{i}$ & 1.1 & 10 & 0.6 & $11 . i$ \\
\hline $18 \% \ddot{3}$ & 0.9 & 0.6 & 0.7 & 0.1 \\
\hline 1876. & 0.9 & 11.7 & 0.5 & $n$ \\
\hline $187 \pi$ & 1.4 & 1.1 & 1.0 & 11.3 \\
\hline 187. & 1.1 & 1.0 & 1.1 & 0.2 \\
\hline 1879 & 1.9 & 1. & 1.11 & 11.8 \\
\hline 1880 & 2.0 & 1.7 & $1 . i$ & 11.2 \\
\hline 1881 & 3.3 & 2.6 & 2.7 & 0. \\
\hline 1882 & 3.2 & $2 .:$ & $2 . x$ & $(1,2$ \\
\hline $18 \times 3$. & 3.7 & 2.6 & 3.11 & $0 . \ddot{z}$ \\
\hline $18 \times 1$. & 4.2 & 3.4 & 3.4 & 19.7 \\
\hline $188 \%$ & 4.4 & 3.3 & 3.8 & $11 .:$ \\
\hline 1886. & 4.1 & 3.1 & 3.7 & 11.3 \\
\hline $188 \%$ & 4.3 & 3.1 & 3.8 & $11 . .1$ \\
\hline 1888 & 7.2 & $\vdots .6$ & $\because .2$ & 1.9 \\
\hline 1889 & 11.7 & 9.5 & $\therefore 1$ & $i . i$ \\
\hline 1890 & 10.5 & 8.7 & 8.2 & 2.1 \\
\hline 1891. & 6.9 & $\because i .1$ & 6.0 & 0.1 \\
\hline 1892. & 10.0 & .8 & 6.8 & 3.1 \\
\hline 1893. & 8.3 & $i .6$ & 7.11 & 1.2 \\
\hline 1894. & 10.1 & b.x & 6.6 & 3.1 \\
\hline $18: \%$ & 18.0 & 13.6 & 8.7 & i. \\
\hline 1890. & 12.1 & 8.9 & $\because .2$ & $\because 2$ \\
\hline 1897. & 14.0 & 111.1 & 4.2 & $3 . \ddot{i}$ \\
\hline 1898. & 16.8 & $1: 3.11$ & $13 . \%$ & $2 . !$ \\
\hline 1897 & 19.7 & 14.4 & $17 . t$ & $\because 11$ \\
\hline 1900 & 19.5 & $1 i .11$ & 14.2 & 11.! \\
\hline 1901 & 13.9 & 9.8 & $1 \because i n$ & $-11 .$. \\
\hline $1+11) 2$ & 13.4 & 13.6 & $1 \mathrm{it}$ & i. li \\
\hline 1903. & 13.8 & 13.9 & $1 \therefore .7$ & 3.11 \\
\hline $190 \dot{t}$ & 23.9 & 16.6 & $1 ! 1.1$ & $i .$. \\
\hline
\end{tabular}

Produit des alfaires de banque réProduit les titres el artian- 
DEUXIENE PARTIE. - CHAPITRE IV

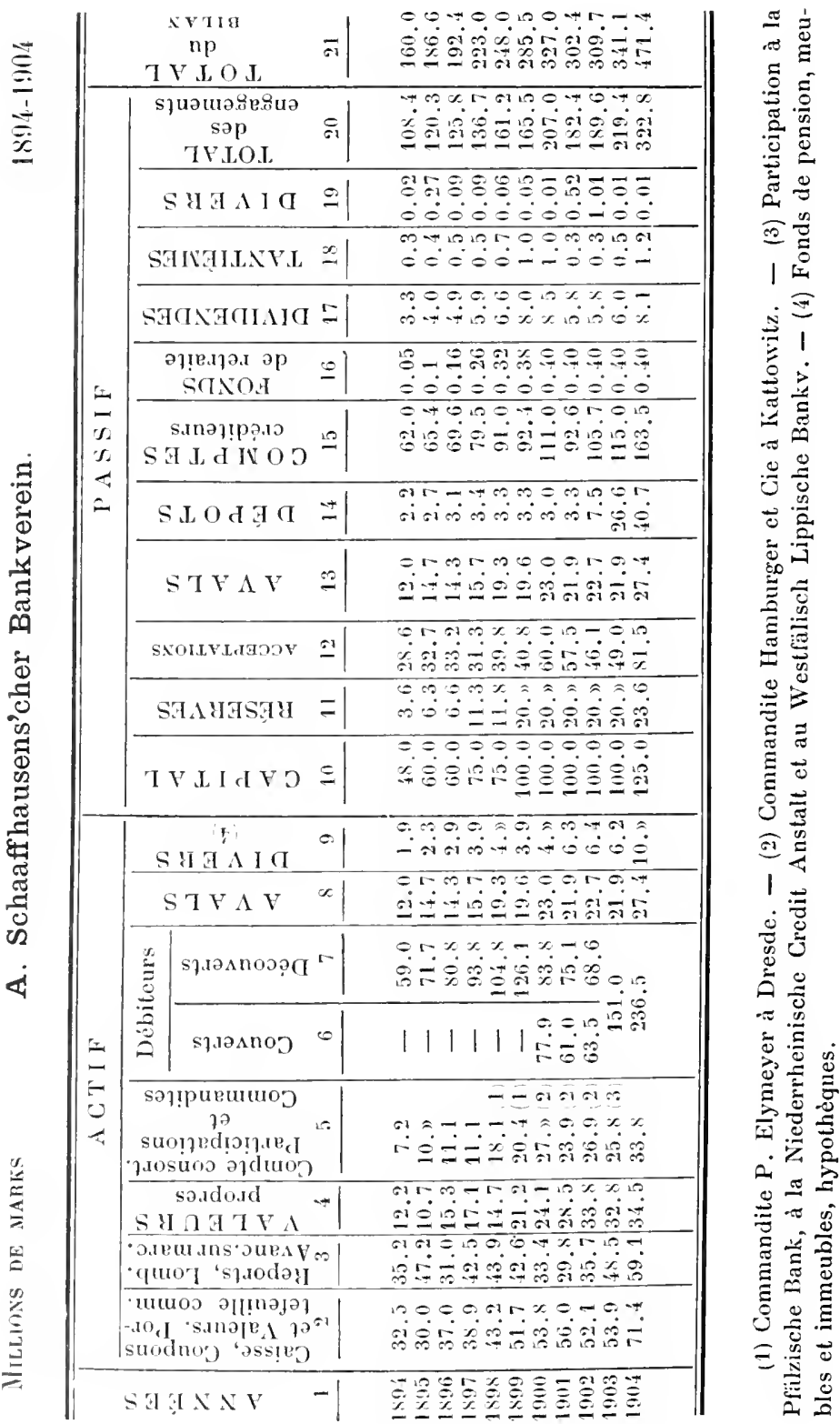




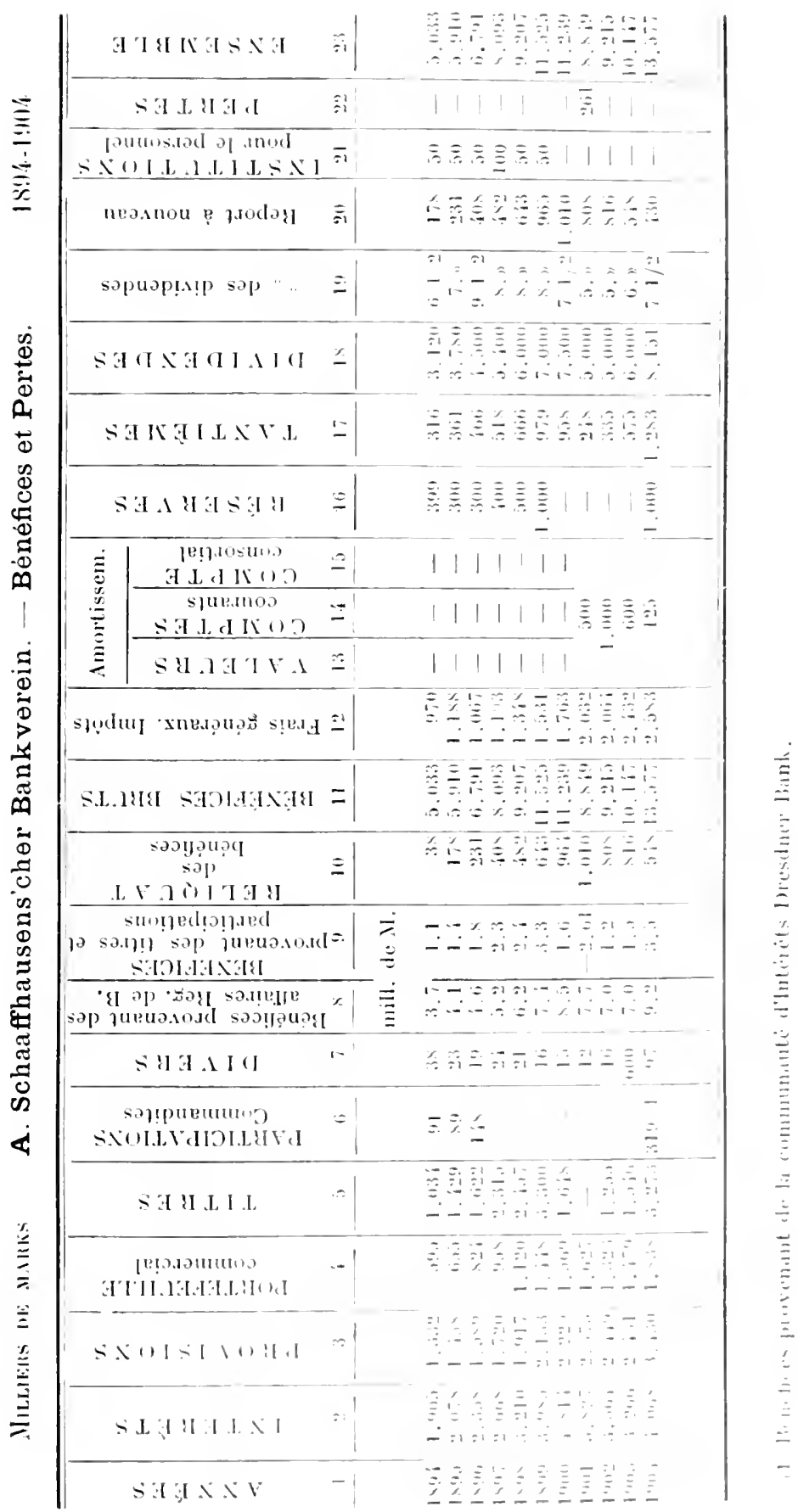




\section{II. - La formation du Groupe Drestner Bank -} A. Schatfhausens'eher Bankverein.

A. - La Dresdner Bank a successivement fondé les succursales snivanles: Berlin (1881); Ilamboury (1892), par absorption de l'Anglo-Deulsche Bank, capital 12.300.000 marks ; Brêne (1895) par absorption dי la Bremer Bank, capital 20.000.000 de marlis; Nuremberg(1896), par absorption de la maison S. E. Wertheimber; Fürth (1896); Hanovre (1898), par absorption le la maison Alexandre Simon ; Mannheim (1899); Chemniz (1899) ; Bückeburg (I899), par absorption de la Niedersächsischr Bank; Lü. beck (1900) ; Altona (1900) ; Londres (1901) ; Detmold (1901); Zwickau (1901); Francfort (1904), par absorption de la maison Erlanger et fils; Munich (190:i).

Elle possède 18 caisses de dépòts à Berlin et aux environs,

3 caisses de dépòts à llambourg

1 - dans les villes suivantes: Altona, Detmold, Dreste, Emden, Francfort, Hanovre, Lïbeck. Elle commandite la maison Bosse, Keil et Cie, à Berlin.

La Dresdner Bank a participé à la fondation des Sociétés filiales suivantes :

1889. Deutsch-Asiatische Bank.

1894. Banca Commerciale Italiana.

1904. Deutsch. Westafrikanische Bank.

- Actiengesellschaft von Speyr et $C^{0}$, à Bàle (capital $1 \% .000 .000$ de francs).

1908. Tremhand Ierrinignung Actiengesellschaft, à Berlin (avec l'unique concours de l'A. Schaaffhausens'cher Banl:verein), capital 1.000 .000 de marks. 
Constitution du groupe de la Dresdner Bank.

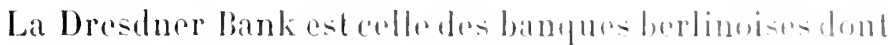

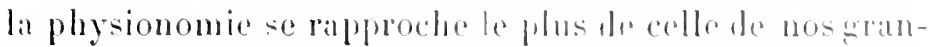

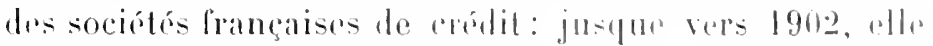
s'est bornée à absorber purrment ed simplement bes ban.

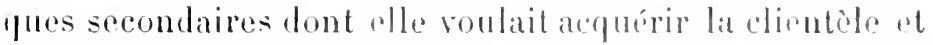

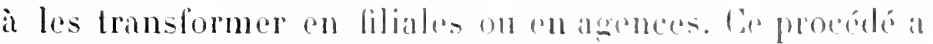
nécessite des angumentations de cilpital comsiderables.

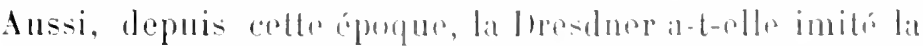

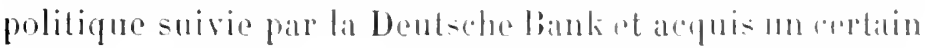
nombre deaclions dans las institutions quiolle derirat

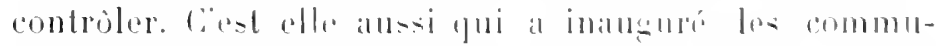

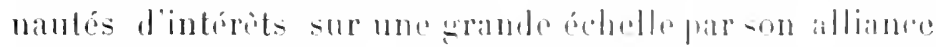
contracturlle are. l'A. Schaaffhausens'cher Bankverein.

1) Absorptions de la lirestuer lirmli.

1872. Michacl liaskel, à lleste.

1S73. Sächsischer bankveroin.

1877. Siichsisolue Crodithank.

1s!1!. R. Thodret tiv.

1892. Anglo Deutsche Bank, to Ilamlonro. La [lrealner

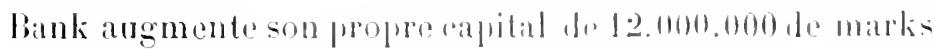

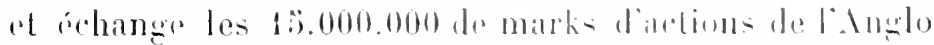

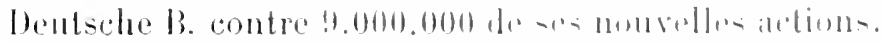

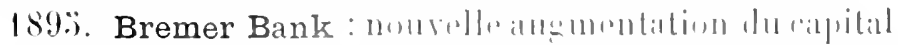

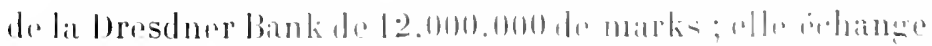

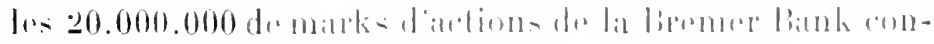

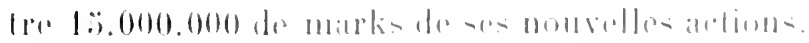

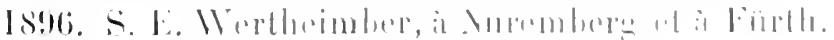

1

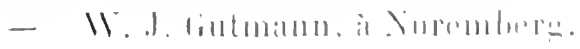


La Dresdner Bank ćlève son capital de $2 \ddot{3}$ millions, fin 1896 , pour réaliser ces absorptions.

1899. Nouvelle augmentation de 20 millions de marks; 4.500.000 marks de ces nouvelles actions sont échangées contre les $\mathbf{6 . 0 0 0 . 0 0 0 ~ d e ~ m a r k s ~ d ' a c t i o n s ~ d e ~ l a ~ N i e d e r s i i c h - ~}$ sische Bank de Bückeburg.

1902. Eduard Rocksch.

1904. La Dresdner Bank augmente son capital de 30 millions de marks et s'incorpore:

a) Ia maison Sorgel Parisius et Cie, Deutschegenossenschaftsbanli, à Francfort et Berlin, dont elle acquiert les 30 millions de marks de capital moyennant 20 millions de marks de ses propres actions et 3.730 .000 marks payés en espèces.

La Deutschegenossenschaftsbank avait absorbé : 1899. La maison Breunig et Fischer, à Stuttgart. 1900 "A. Schmidts, à Heilbronn.

Elle possédait 1.000.000 de marks d'actions environ de la Wiirttembergische Landesbank (Stuttgart), capital 6.000 .000 de marks.

b) La maison Erlanger et fils de Francfort (avec le concours de l'A. Schaaffhausens'cher Bankverein).

Cette maison était elle-mème intéressée, par possession d'actions dans:

$1^{\circ}$ L'Oldenburgische Landesbank (capital 3.000.000 de marlis).

$\because$ filiales.

43 agences.

$2^{\circ} \quad$ La Mecklemburgische Bank, à Schwerin (capital 5.000 .000 de marks).

5 filiales.

43 agences. 
Elle-mème en communauté dintérèts avec la Rostocker Gowerbebank (capital :2.060.000 de marks.

11 agences.

et la Neuvorpommersche Spar und Creditbank, it ilrill. sund capital 1.000 .000 de marks, sougences.

La Dresdner Bank el li. Schaaflhausenscher likv. sont representées dans le conseit de la Meclilemburgische $\mathrm{b}$.

30 La Landgraflich Hessische Landesbank à $110 m b u r g$ (capital 1.000.000 de marks).

1 filiale.

to La Schwarzburgische Landesbank, a sondershancen capital 2.800 .000 marks).

50 Eisenbahn Renten Bank capital b.230.000 mark-

$6^{\circ}$ Eisenbahn Bank capital $\mathbf{4} 000.000$ de marlis.

7o Frankfurter Hypotekenverein capital 10..;100.41\%) .1. .

$8^{\circ}$ Schwarzburger Hypotekenbank (capital 2.7.69.090 . . .

Toutes cesbanques font maintenant partiodu concret de la Dresiner Bank.

B) Participations par possession dactions.

a) La Dresdnor Bank a alequis on 1903, 1.000.000 de marks d'actions de la Mærkische Bank dr. Bochun dont le capital a été porté en 190\% de 6.000 .000 dr marks a 8.000 .000 .

Cette banque ne date que de 1898 : elle possède 8 aqrences et a absorbé :

1898. La maison A. Lauffs, it Bochum.

1899. La Herner Bank cápilal 1.000.001) de marki-

b) Elle a adde en l90:3, il la reconstitution do la Rheinis-

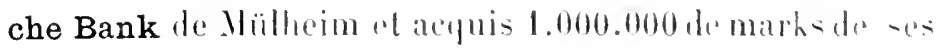
aclions.

Le capilal de la liheinische liank a éle porté en lono: de lo a

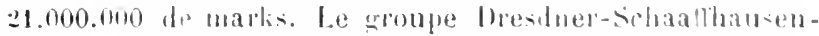

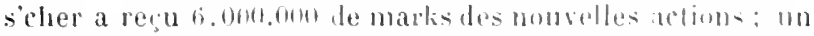
échange doudministrateurs a eu lims entre les dear huques. 
La Rheinische Bank possède 2 filiales.

Elle a absorbé en 1897 la maison G. Hanaı à Mülheım.

Le siège de la banque a été transféré en 190 à à Essen.

c) Enfin en 1904, la Dresdner a reçu 1.000.000 de marks de nouvelles actions de l'Oberschlesische Bank de Beuthen.

fondée en 1895 et dont le capital primitif 1.500 .000 marks a été porté à 2.000.000 de marks en 1901,

3.000 .000 de marks en 1904 .

Cette banque possède 1 filiale à Konigshïlte.

C) La Dresdner Bank est alliée contractuellement (communauté d'intéréts proprement ditej) à dater du 1 er janvier 1904 àl'A. Schaafthausens'cher Bankverein, à Cologne et Berlin.

B. - Nous avons donné plus haut les bases de cet ac. cord. Montrons la constitution du groupe A. Schaaffhausens'cher Bankverein.

L A. Schaaffhausens'cher a successivement fondé des filiales à Berlin (1891); Essen (1901); Düsseldorf (1902); Bonn ; Crefeld, Neuss, Rheylt, Ruhrort et Viersen (1904).

Il possède 14 caisses de dépôts.

Il commandite depuis 1898 la maison Philipp Elimeyer, à Dresde.

L'A.Schaaffleausens'cher Bankverein a fondé ou participé à la fondation des Sociétés filiales suivantes:

1889. La Deutsch Asiatische Bank, avec le concours des autres grandes banques berlinoises.

1898. La Banque Internutionale de Bruxelles (id.).

1900. La Westfælisch Lippische Vereinsbank de Bielefeld réalisée par la fusion des maisons Katzensteine fils, à 
Bielefeld, Sickmann frepes, it llerford, Salomon et llprenheimer, à Lemgo ot Delmold.

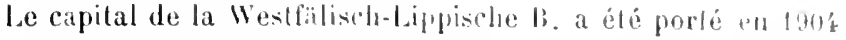
de 3 it 7 millions de marks.

1904. La Société par actions com Speyret Cie, il billo. avec l'unique concours de la llrosdnor Bauk.

190:3. Et entin avec er mome conconrs, la Tientemd Vereinigumg-leliemyesallswaft, il lierlin.

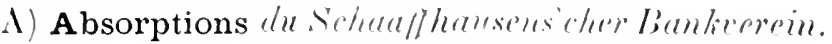

1903. La banque I ot L. Camphansen, à Cologne.

190'. La Westdeutsche Bank (anciommenaison J. Lalın), a Bonn, au capital des.000.000 de matks, qui avait absorbó clle-mème la maison solmile et Cahen en 1 s98. Le capilal de la Westreutsche Bank était We 8.600 .000 marks; la totalite de ces actions lut reprise prar l'A. Schaalfhams. qui donna en ichange anx aclionnaipes 3.733 .000 marks de ses nouvelles actions.

1904. La Niederrheinische Credit Anstalt, i Crofeld, dont les 21.000 .000 de markis d'actions furent échangés contre 1\%.000.000 de marks de nourelles acetions du schadfhans.

Gette banque possindatit 12 filiales.

1904. La maison Erlanger et fils dr. Francfort, aree lo concours de la breadner biank.

B) Participations par possession d'actions.

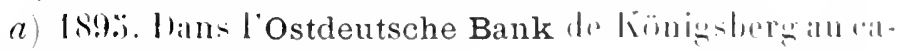
pilal de 10.0100 .000 (10 millis.

Elle possède :2 lilialís

et a deja absorbé la mation J. Simon ot fils, at lïnigsterg el Friedlander il lirumlir.t.

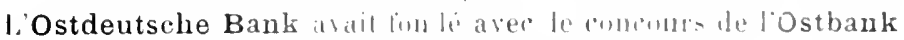

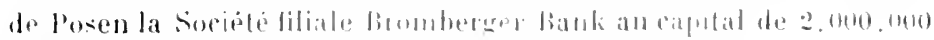

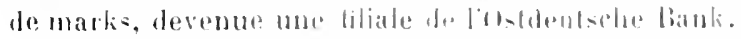

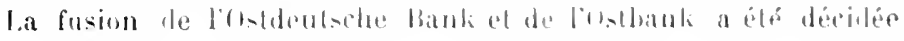


en août 1903 ( Koir Groupe de la Bank für Handel und Industrie, p. 187).

b)L'A.Schaaffausens'cher possède depuis 1901,2.600.000 marks d'actions de la Pfælzische Bank de Ludwigshafen. fondée en 1883 au capital de 600.0060 marks.

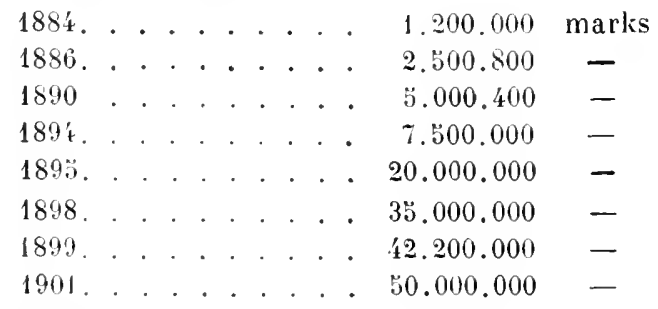

Ces élérations de capital ont été faites pour favoriser les absorptions suivantes:

1886. La Volksbank, de Ludwigshafen.

1894. Joh. Franck, à Worms.

- Louis Dacqué, à Neuestadt.

1895. Deutsche Union Bank, de Mannheim qui avait ellemème absorbé en 1891 la maison Sonneberg frères de Francforl.

1896. J. F. Haid, à Spire.

1897. Muller et Weyland, à Landau.

- Hermann Meuner, à Landau.

1898. Bloch et Cie, à Nunich et Nuremberg.

- Pichlerssel, ì Munich.

1899. Le Vorschussverein, de Bamberg.

- Le Vorschussverein, d'Alzey.

1900. M. Levy, à Worms.

La Pfälzische Bank possède 16 filiales,

† agences et caisses de dépôts.

c) L'A. Schartfhausens'cher Bankverein possède depuis $1903,3.000 .000$ de marks d'actions de la Mittelrheinische Bank, fondée en 1873 à Coblenz: capilal 9.000.000 de marks porté en 1903 à 12.000 .000 de marks

Cette banque est intéressée elle-mème à la Mülheimer Bank (capital 7.500.000 marks, qui a deux agences. 
La Hiltelrheinische Bank possède 2 liliales. Elle envoie un llirpeteur an conseil dadministration du sichatihasencicher Bankwein qui de son côté a un délézué au conseil de la Vlittelmeinisclue lank.

Le tableau suirant montre les bínélices que la Dresilur Bank et l’A. Schathausens'cher ont pu retirer de lomp participations (dividendes distribués par les banques alliliées).

Mirkische Bank.

Oberschlesische liank.

Wurttembergische

Lan-desbank. .

Oldenburgische. . .

I ecklembiurg ische

Bank. . . . . .

Landgräflich Hessische

B........ .

Schwarzbùrgische Landesb. . . . . .

Ostdeutsche Bank. .

Pfilzische Bauli. . . . $\begin{array}{llllll}1893-1896 & 1897-1900 & 1901 & 1902 & 19913 & 190: 3\end{array}$

Mittlelrheinische Bank.

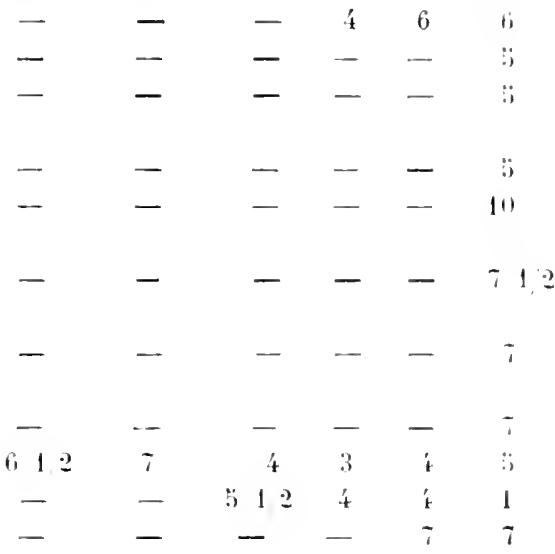

III. - Les participations industrielles et rommerciales dre

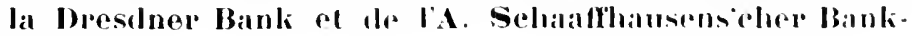
verein.

\section{A. - Dresdner Bank.}

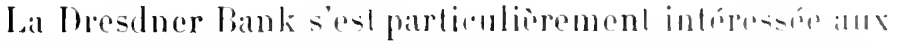
affaires électriques. mais alle possedre igalomont do mom-

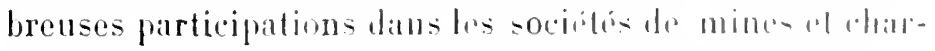
bonnages ef dans les suciétés die comstruetion de maehines.

Elle se trowvat en 190's avoir un le ses membres diroe- 
teur dans le conseil d'administration des entreprises suivantes (nous soulignons celles des entreprises auxquelles la Dresdner Bank s'est plus spécialement intéressée [fondation ou transformation]):

$1{ }^{\circ}$ Mines el Charbomages:

A. G. Lauchhąmmer (Riesa).

Deutsch Osterrheinische Bergwerksgesellschaft (Dresde).

Sun mal Mosel Berguerksy. (Spittelkarlingen).

Schlesisch. A. G. für Borgbau und Zinkhütten betrieb (Lipine).

Steintiohlen bergererti Nortstern (Wssen).

Vereinigte Königs und Laura Hülte A. G. für Bergbau und Hüttenbetrieb (Berlin).

$2^{\circ}$ Construction de machines:

Ascherslebener Maschinenbau A. G.

Deutsche Niles Werkzeugmaschinenfabrik (Berlin)

Ludwig Lew et Co (Berlin).

$3^{0}$ Iudustries électriques:

Allg. Elektricitätsgesellschaft.

Deutsch. Ueberseeische Elektricitäts Gesellschaft.

Gesellschaft für elcktrische Unternehmungen.

Leipsiger Elektricitätsueve (Leipzig).

Mexiran Elertric ITorks (London).

Merican Light et Pourer Co Limited (Mlontreal).

4. Industries chimiques:

Chem. Fabrik Heyden (Dresde).

Elle est en outre intéressée aux Sociétés suivantes:

Deulsche Wassen und Munitionen Fabrik.

The Ioynamit Trust Co.

Berlinische Bodengesellschuft (fondation 1898). 


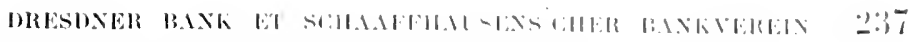

Bodengesellschaft liurfiurslondamm.

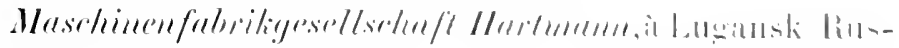
sie) (Pondation 1896 ).

Fetd und Kleinbahnmaterial A. R. (Berlin).

Maschinenfabrik Gobrïder bihlos (iv.

Continental Eisenbahn Bau und betriobsgesellschall.

Anabolisehen Eisenbahn (ave la participalion de hat Deutsche Bank).

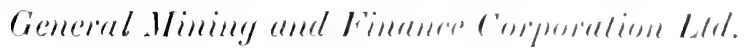

\section{B. - A. Schaaffhausens cher Bankverein.}

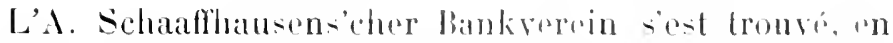

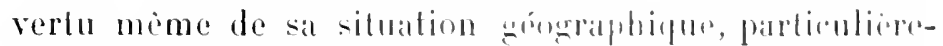
ment intéressé allx mines. charbonnases ot aciories d. la

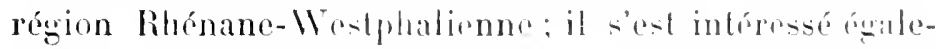
ment aux alfaires blectriques, atux fabriques de textiles el de produits chimigues; ce sunt ees deux dernieres industries surtout qui lui ont causé les grosses pertes ha lexereice 1901 .

Il a un administrateur délioné dans chacune des entreprises suivantes:

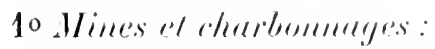

Bergball A. G. (Massen, Niedernassen).

Berowerksgesellschatt Trier).

Bergwerksveroin Frielerich Wilhelms llütle Milloim. Eschweilor Köln Eisenwrik.

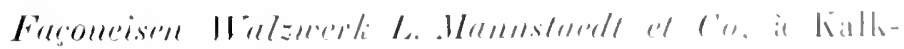
Colosne (transformation a X. 1i.).

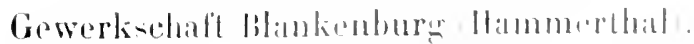

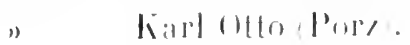

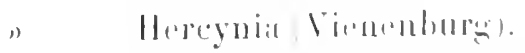

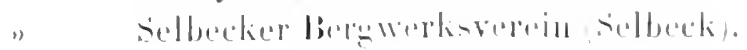


IIarpener Bergbau A. G. Dortmund.

Ilauts fourneaux lorrains-Aumetz "La Paix »

II"̈rder Bergwerls und IÏ̈ttenverein.

Internationale Berggesellschaft (Erkelenz).

Jekaterinoslawer Röhren und Eisenwalzwerk.

Lö̈ner Bergwerksverein (Altenessen).

Oberschlesische Eisenindustrie (Gleiwitz).

Phönix A. G. für Bergbau und Hüttenbelrieb (Laar).

Schalker Gruben und Ilüttenverein.

Schwelmer Eisenwerk Muller et Co A. G.

Sieg-Rheinische IIïtten A. G. (fondation).

Stahlwerk Krieger (Düsseldori).

Ver. Stahlwerke Zypen und Wissener Eisenhütten (Ciologne-Deutz).

Zechau Kriebitzscher Kohlenwerke (Glückauf).

$2^{\circ}$ Construction de machines:

Benrather Maschinenfabrik.

Berlin. Anhaltische Maschinenbau A. G.

Giasmotorenfabrik (Langen et Wolf).

Kialker Werkzeugmaschinenfabrik (Breuer).

Ludwig Loewe et Co (Berlin).

Maschinenbauanstalt Humboldt (Kalk).

Maschinenfabrik Deutschland (Dortmund).

" (Grevenbroich).

Werkzeugmaschinenfabrik Gildemeister et Co (Bielefeild).

$3^{\circ}$ Industries électriques .

Allg. Elektricitätsgesellschaft.

Gesellschaft für elektrische Unternehmungen.

1. Industries chimiques:

A. G. für Chemische Industrie (Schalke).

Chem. Fabrik Hönningen (Feld). 


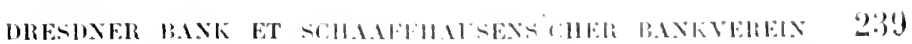

L'A. Schatrhausens'cher likv. s'est en oulre particulicrement intéressé aux entreplises suirantes:

Girande socićté de T'ramways (Berlin).

Société de navigation de Broslau.

Bahler A. G.

British Thomson-Houston.

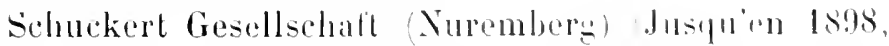
aujourd'hui Loewe et $\mathrm{C}^{\circ}{ }^{\circ}$.

Syndicats Kontors des A. Scleauffle. Blice. fondation $18: 19$. capital 1.000.000 de marks).

Dïlkener Baumeollspinnerei, -_ Rheinische Wehsulelfabrik (fondations 1597).

Sï̈deutsche Juteimlustrip (Mannheim), fondation 4.000 .000 de marlis, capilal.

Lammgarnspimerei und WVeberi Eitorf (transformation en A. G. 1901, capital 2.3̈00.000 marlis).

La Dresdner Bank et l' 1 . Schathluasens'cher Bankrelein font lous deux partic du Consorlium de l'russe: c'est dire quils ont pris une part actire a toutes les ćmissions d'emprunts d'Etats allemands el étrangarers.

Les tableaux suivants, empruntés aux rapports généraux de 190', montreront l'activité des deux banques sur ce terrain des émissions:

\section{Dresther Banli:}

Emprunt 31200 de l'Empire allemand et de l'Elat prussien.

31200 le l'Etat batrous.

$: 1200$ lusinode de Berlin.

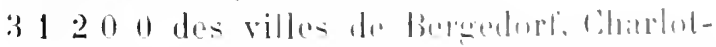

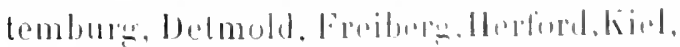

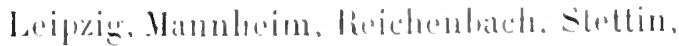
l'anin. 
Emprunt $33 / 40 / 0$ de la ville de Linden.

" $31 / 20 / 0$ (obligations) du Braunschweig, Landes Eisenbahn Gesellschaft.

" $\quad 40 / 0$ (obligations foncières) de la Deutsch. Grund Credit Bank.

$33 / 4$ et 400 (obligations foncières) du Frankfurter Iypoteken Credit Verein. des Creditkasse.

)

400 (obligations foncières) de la Preussische Hypoteken Actienbank.

" $\quad 40,0$ (obligations foncières) de la Rheinisch Westfälische Boden Credit Bank.

" $\quad 33 / 400$ (obligations foncières) du Sächsische Boden Credit Anstalt.

" 40/0 (obligations) de la Deutsch. Niederländische Telegraphengesellschaft.

4 1/20/0 (obligations) du . Nülheimer Bergwerks-Vereins.

วั 0 , 0 de la Tebuanteper National Eisenbahngesellschaft.

Actions de la Banque d'Entreprises Electriques, à Zurich.

Berlinische Boden Credit Aktien Gesells chaft.

Nouvelles actions de la Deutsch Asiatische Bank.

"

)

) de la Deutsch Westafrikanische Bank. du Sächsische Boden Credit Anstalt. de l'Allgemeine Elektricitäts Gesellschaft.

de la Neue Maschinen Fabrik Kyffhaiiserhütte (maison P. Reuss), à Artern. 
Actions de la Compania explotantora lo canchu moxicano.

Actions de la Lhemische Fabrik ron llevelun .1. li.

Participation nouvelle dans la Deutich Wratrihani-che Handelsgesellschaft.

Actions de la Deutsch Niederbindische Telendalen Gesellschaft.

Nomvelles actions de la Deutsch-Lbersecische Eloktricitäts Gesellschalt.

Nouvelles actions do la Gelsenkirchener Burgwerks Gesellschaft.

Nourelles actions des grands tramways berlinois.

Nouvelles actions de la Ilannoversche Aktien Gummi Waarenfabrik.

Actions de la maison kärting frères A. G.

Actions de la Kammgarnspinnerei-schedewitz A. r.

Nouvelles actions de la IIarpener Berghau Gesellschalt.

Actions de la Rïtgerswerlie A. G.

Actions de la Petrolem 1. G. Campina-Moreni.

" Societa Italiana per la liabricasione dull' $\mathrm{Al}$ luminio ed altri prodalli delleleltrometalluryia.

„Terraingesellschaft du Ceatral-Miehlof.

Nouvelles actions de la Winderer-Faluradwerke-Winklohfer et Janicke A. G.

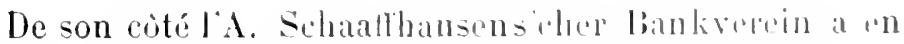
1904. participé aux émissions suranter:

Emprunt 312011 de l'Empire allemand on de listat prusinds.

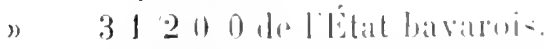

" 3120 (t) la provincm dis libin. 


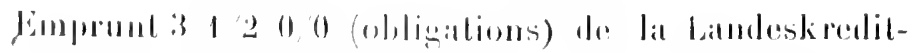

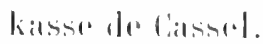

":31,20,0 des villes de Berlin (Synode), Chatrlollembury, Dortmund, liel, Leiprig, strllin.

4. 11 0) (ubligations) de la Preussische Ilypoteken Actienlank.

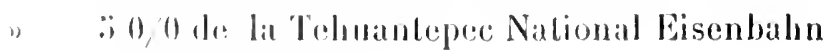
riesellschtialt.

" $\quad 1,200$ (obligitions de la " Aller " Deuts-

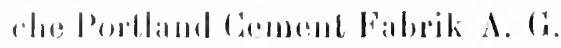

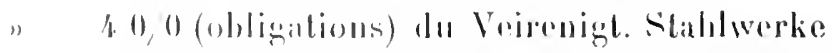
vitn der zypen und Wissener lisenhïtlen, A. 1i.

"40 (obligations) de lat Deutsehe Dbersecis(lar litentricitits $\Lambda$. R.

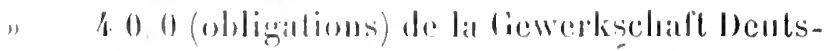
rher Kistiser.

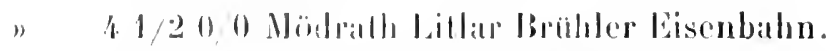

" 1,200 (obligitlons) du Miilleeimer Bergwerksvererins.

" 4. (1) (obligations) du Schalker Gruben und Iiillen Vereins.

" $41 / 20 / 4$ (obligations) du Zechau Kricbitzscher kohlenwerke "Gilichatul" ".

Nonvellos andions do l'Allgeneine Elektricititsgesellschallt.

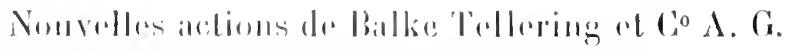

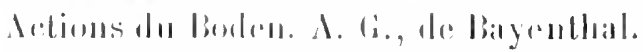

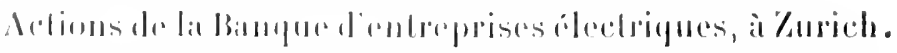

Nonvollas actions da la biank liïr llandel and Industrie.

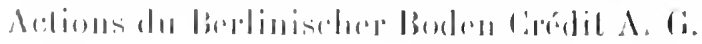




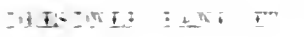

$-1$

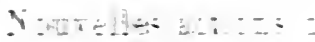

$\because=-11 \div-\therefore-\therefore$.

t.

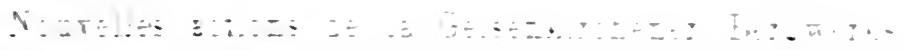

$\therefore i$

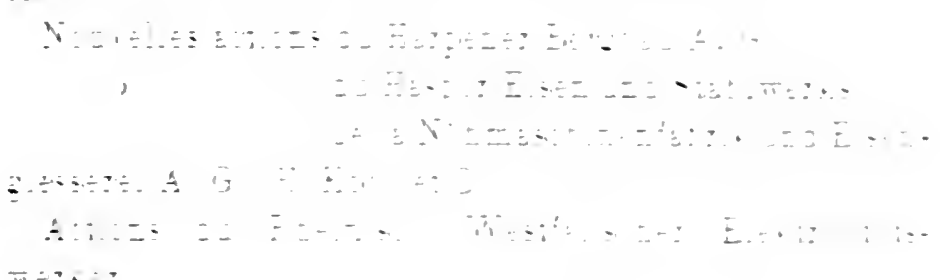

$\overline{10} \div 2 \div$

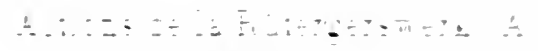

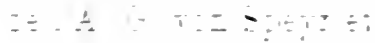

$\Sigma \because \because \div$

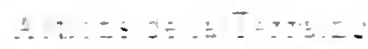

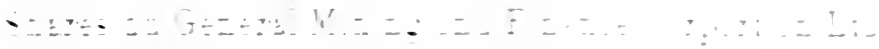

L

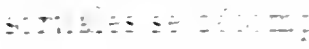

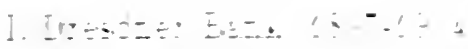

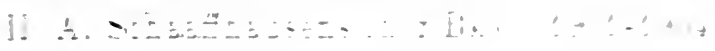


DEUXièle PARTIE. - ChAPITRE IV

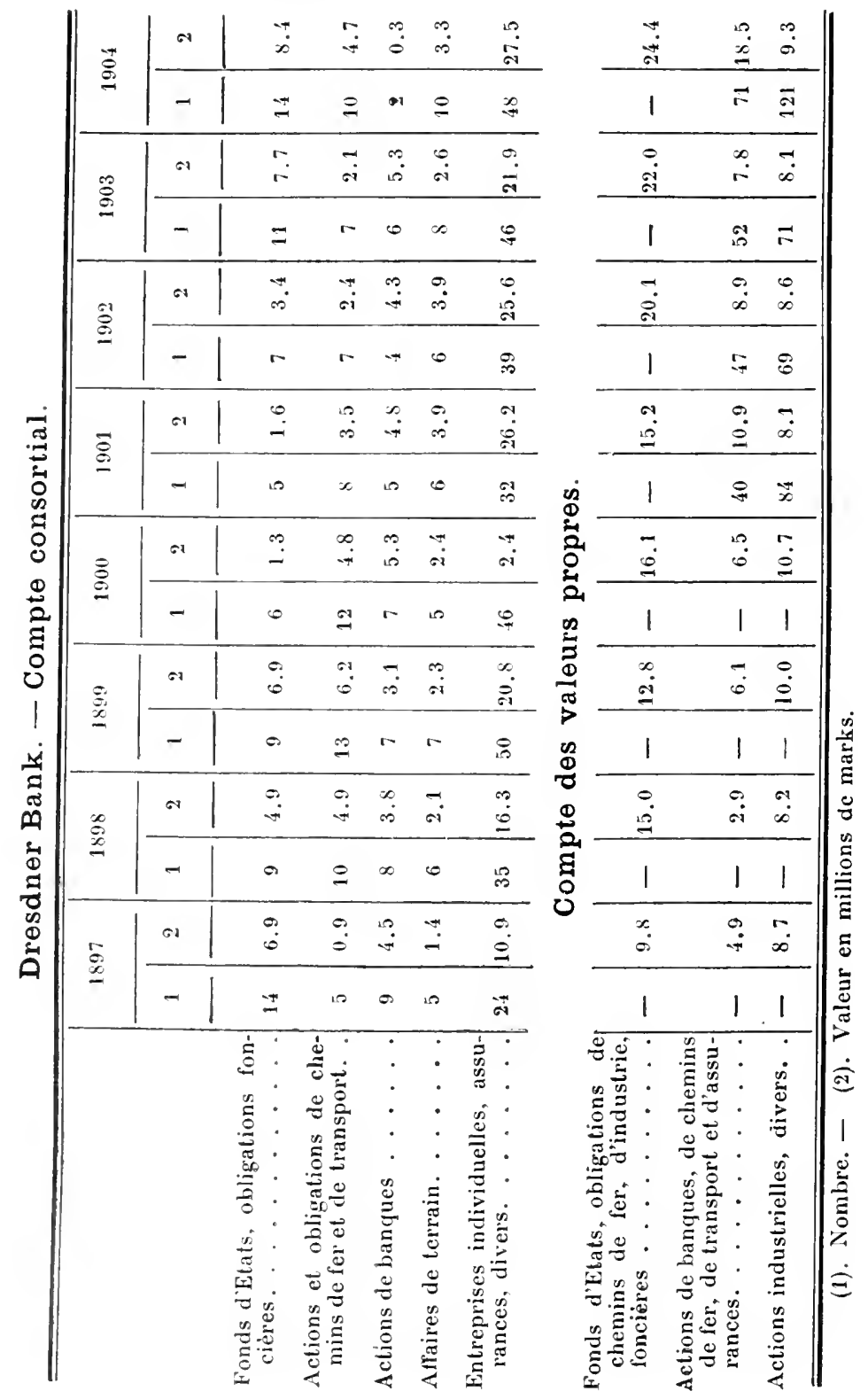




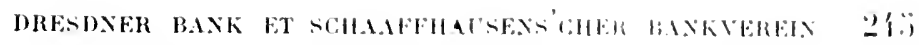

1. Schaaffhausensicher Banliverein.

Comple consmilial.

$$
1901 \text { millions de marks } 19019
$$

Actions de banque et de Compagnies

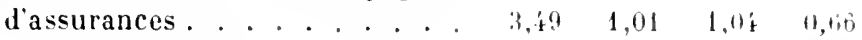

Actions et obligations d'entreprises

de transport......... 4, 4, $4: \$, 99 \quad 6,26 \quad 7,80$

Actions et obligations d'entreprises

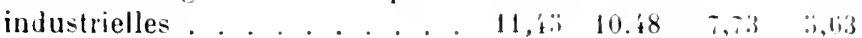

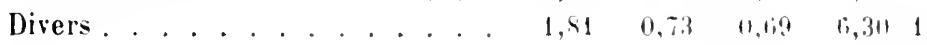

Comple des caleurs propres.

Fonds detat. M!nizations foncires

et industrielles. . . . . . . . . T T,12 9,71 \$, it 9,19

Valeurs cotées en bourse. . . . 13,10 10,29 10,63 1:2,311

Valeurs non cotés en Bourse. . . 8,2 13,30 1:, 1:2 13,22

(1) Dont '́, isi,000 marks de fonds d'Etats allemands et chblizillions foncieres. 



\section{CONCLISION}

The lexamen de tous ores chillese ut de tous ces faits ressortent bien nettrment les idres principaldes qua mons nous sommes ellowe de metlre en lumiere dans lis premiere partie de ce travail.

Mieux que des résultals l'ensemble ou gun l'exposition

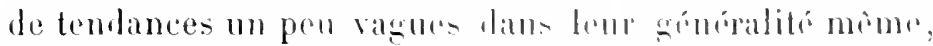
les bilans particuliers de charme de grandes hanques nous ont révélí l’intime conlusion an sein des lanques allemandes de ces différentes fonetions que partout ailleurs nous trouvons solghensement listingures, - fonc-

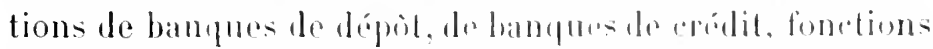
de banques draffaires. - Sims donte. les grands élalulis-

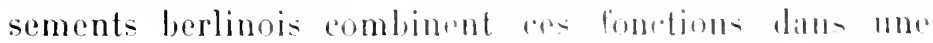

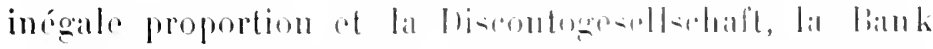

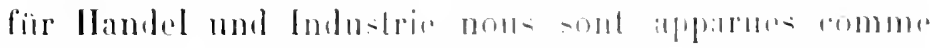

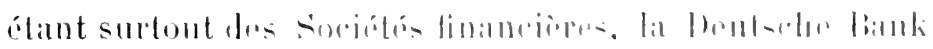

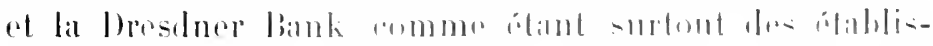
sements de crélit. Matis on a vall

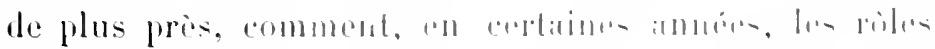

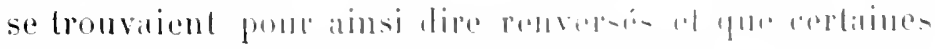

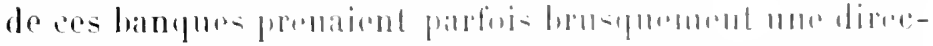

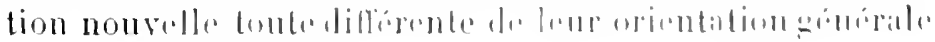


ct accoutumée; et ceci nous montre à quel point sont étroits les rapports de ces grands Instituts berlinois et des conditions économiques du pays tout entier. On a dit que de tous les problèmes que soulève l'organisation éco. nomique d'un pays, il n'en était guère de plus importants que ceux relatifs au régime de ses banques. Nous voyons comment en Allemagne, celles-ci indiquent les moindres variations du marché national, comment elles s'efforcent de les prévoir ou de s'y adapter, redoublant leur activité pendant les périodes prospères, au contraire se repliant sur elles-mêmes pour laisser passer l'orage. C'est done à juste titre que nous avons pu ranger au nombre des causes générales du mouvement de concentration et cette façon mème de comprendre et de pratiquer la banque, - et cette union intime de la hanque et des autres manifestations de l'aclivité économique que l'examen détaillé des participations industriclles et commerciales des grands ćtablissements est venu singulièrement éclairer.

lci, les conditions locales, les relations particulières des hommes placés ì la tête des grands établissements ont sans donte décidé en partic des sortes particulières d'industries auxquelles allaient s’intéresser les banques. La Dentsche Bank s'est plus spéeialement altachée au développement des affaires électriques et du commerce transallantique, la Discontogesellschaft s'est intéressée aux entreprises de transport, la Dresdner Bank aux industries textiles etchimiques, l'A. Scharfhausens'cher Bankverein aux mines et aux houillères : la Darmstädter Bank seule ne paraît point s'ètre spécialisée. Mais l'ensemble des participations industrielles et commerciales des grands établissements embrasso toutes les industries fondamentales de l'économir nationale; nous en arons vu la somme - qui 
pour 1904 ne reprósente pas moins de $891 \%$, 6 millions le

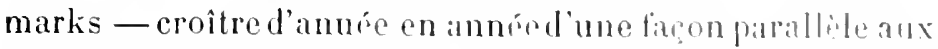
progrès de lindustrie et du commerce allemandi. Il sitwit donc bien là d'un fait général dont mons lovions drugener les conséquences logiques.

Laction des causes particulieres gue nons avoms signalées nest pas moins manifeste danschacun do ees átablicsements: on a pu noter la nourelle vigueur qua priar id pariir de 1896 - loi sur le Drpot ot sur les lourses - Io reguläre geschïft et limportant aceroisoment de la plu. part deschapitres. Laction de la crise 1900-1901 n e-t pats moins évidente à cet égard. Les enseignomonts qui sen sont digagés, danger d’immobilisations trop ensidéril bles, nécessité pour divelopper los opérations do bamqua ordinaires de s'étendre el de rayouner, ont imprimó a la [iscontogesellschaft, voire a la Darmstädter Bank uredirection nouvelle comme aussi bien ils ont fortifir la leut-che Bank et la Dresdner bank dans une ligne lo conduito que déjà elles prétendaient suivro.

D'autre part nous avons retrourí plus nu moins utilisés par chacun des grands ótablissementi din- lo processus de concentration res moyms lont nous arome

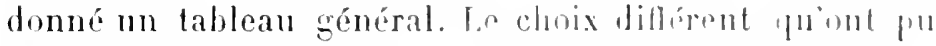
faire de ces méthodes les Instituts bolinois - ixplinur ici encore par des considerations particuliepes fur nou-

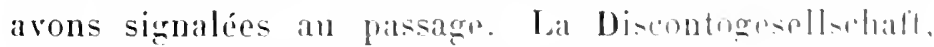

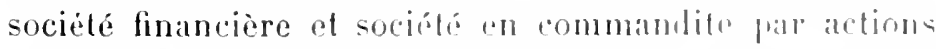
n’a décidé de difluser son aretiviti par rasonnement is travers les provinces gur du jour oì los merentés de la concurrence et les conditions économiques de l' Allemagne 
l'ont forcée à développer ses opérations de banque pure. Longtemps la Darmstädter Bank a cru suffisant le procédé des commandites; la Dresdner Bank a longtemps pratiqué le mode des absorptions pures et simples. Mais il faut remarquer la tendance qui paraìt animer en ces toutes deruières années les grands établissements et les pousse à employer de préférence le mode des participations par achat ou échange d'actions à l'exemple de la Deutsche Bank; nous connaissons les avantages techniques de ce procédé et cette politique n'a sans doute pas peu contribué à assurer la supériorité et la prépondérance actuclles de la Deutsche Bank.

Peut-être aussi verrons-nous se généraliser les communautés d'intérêts contractuelles dont l'alliance Dresdner Bank-A. Schaaffhausens'cher Bankierein nous fournit un exemple si précis. Ce que paraissent en effet chercher avant tout les grandes banques allemandes cest une diffusion de leur action, un complément de forces qui n'entraìne point de grandes immobilisations et qui leur laisse, comme aussi bien aux banques alliées, une large liberté d'action nécessitée par les conditions économiques mèmes de l'Allemagne, une grande faculté d'adaptation aux besoins régionaux Les Interessengemeinschaften paraissent répondre à tous ces desiderata.

Enfin, l'unité des causes dı mouvement de concentration dans les banques allemandes qui se révèle dans la multiplicité de ces faits de concentration que nous avons relevés et dont le nombre et l'importance s'accroissent d'année en année - non moins que dans les augmentations du capital propre, que nous avons tenu à délailler, tant pour les grands Instituts que pour les banques secondaires, prouve assez qu'il s'agit bien d'un 
mouvement génélal, manifestation de liun des grand couranti qui agrisent dans les suciétés modernes - h. capitalisme " 1 - et qu'on ne saurait trop simplement ramener à laction de causes toutes individuelle-et contingentes.

1 Bourguin, op. sil p. 130. 



\section{BIBLIOGRAPIILE}

\section{Bibliographie grinibale.}

Bernstein Edouard). - Socialisme thiorique et social-lémocmatie pratique. L'raduction Mexandre Ciohen. Paris, 1900.

Blondel Georges: - Liessor industriel et commercial du peuple allemand, 3 édition. Paris, 1900.

Bourguin (Maurice. - Les systimes socialistes et lévolution économique. Paris, 1901.

Caurès Paul). - Cours déconomie politique, 3e édition. Paris, 1893.

Courcelle-Seneuil. - Les opérations de banyue, ge eitition, revue par André Liesse. Paris, 190.).

Lexis. - Arlicle Banti dans te go Supplement 1897, do Hamherirterluch der studsucissenswaften (Comad.

Marx Karl. - l.e capital, 1. I : traduction thoy Paris, 1st3: 1. Il. III, IV. Paris, 1900-1902. Trad. I. Borehardl et II. Vanderyed.

- Minère de la Philosophie. Paris, 1s!hi.

Schaeffle. - Das zesellschafliche System der mensohliseben Wirts. chaft. Tübingen, 1873.

Scharling (W.). - Baukpolitili. Lima. 1900.

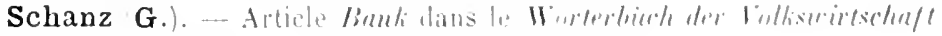
Elster/. 18gs.

Schmoller G. - Princifes d'économie politinge. Trantuction Platon. Paris, 1905.

Sombart (W.) - Ler moderne liapitalismus. Leiprife, 1912.

- Die Deutsche Volkswirtschaft im 1!r Mahrumbert. Merlin. 19013.

Sorel G.). - Introduction a l'économie muderne. Paris, 1!20. 


\section{2) Bibliographie spéciale.}

a) Ouvrages spéciaux, articles et monographies.

Arnauné (A.). - La Monnaie, le crédit et le change, 2e édition. Paris, 1902.

Arnold. - Voir Stärungen.

Aupotit. - La vie financiere, (Revue économique internationale, maijuin 190's, aoùt-septembre, 1905).

Bagehot (W.). - Lombard Street ou le Marché financier en Angleterre. Paris, 1874.

Conant (C. A.). - The Evolution of moderne Banking (Political Science Quaterly, décembre 1899, p. 569).

Dolléans Edouard).- De l'acraparement. Thèse, Paris, 1902.

Eberstadt (Rud.).- Die gegenwärtige Krisis ; ihre Ursachen und die Aufgaben der Gesetzgebung. Berlin, 1902.

Fleischhammer (H.).-- Centralisation im Bankwesen in Deutschland (in Schmollers Ichrbiachern, Iahrgang $25(1901)$, Heft 2, p. 241-270).

François G.). -... Les banques anglaises (Revue d'économie politique, juillet 190:).

Goldschmidt (Ernst). - Die Bankgruppen. Ein Blick in die Concentrationstendenzen der Kapitalistischen Wirtschaftsepoche. Mayence, 1901 .

Hackert (L. B.). - Zur deutschen Bankpolitik (Gegenvart, t. 36, 1899, p. 49).

Hecht (F.). - Die Mannheimer Banken, 1870 bis. 1900. Beitrage zur pratischen Baukpolitik, Leipzis, 1901.

Helfferich. - Voir Stömungen.

Heinemann. - Voir Stijemugen

Jaffé (Edgar). - Das Euglische Bankwesen (iu Schmollers u. Serings Staats v. Socialwiss Forschungen. Bd XXIIl, Heft 4), Leipzig, 190.5.

Lafargue (P.). - Les Trusts Américains. Paris, 1903.

Lévy (R.G.). - Les marchés financiers de l'Allemagne (Revue des Jeux-Hondes, 15 novembre 18:97\%.

- La spéculation et la banque Revue des Ieux-1/ondes, 15 février 1895).

- Ranques de dépòts et banques de spéculation (Revue d'économie politique, mai 1903). 
Lewinger. - Voir Strimulen.

Loeb. - Voir P. Moilel. Stmmun'n.

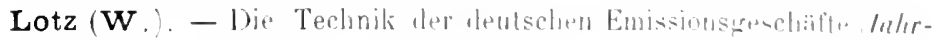

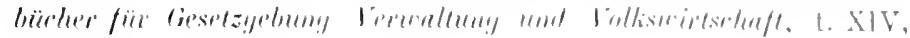
p. 393).

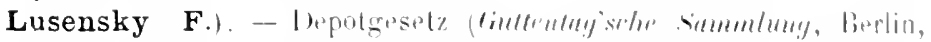
1896).

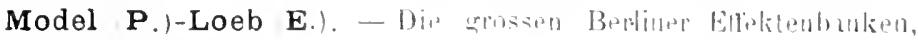
Iena, 1890.

Piekenbrock Carl). - La loi allemime sum les bonures du ?2. juin 1890 el ses ellels. Thise, Lausaume, 16105.

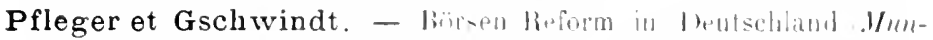

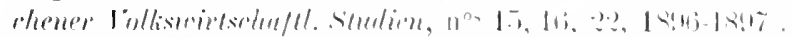

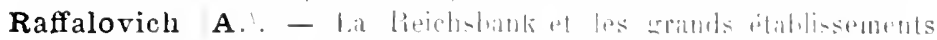

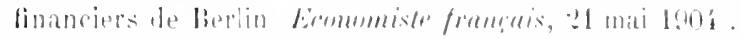

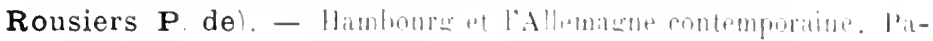
ris, lint?.

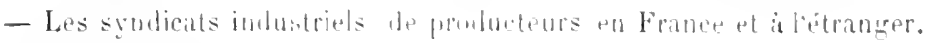
Pitris, 1!n!.

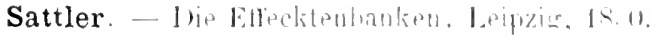

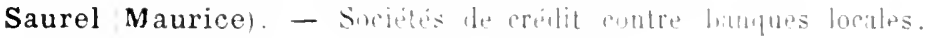
These. Paris, 1901.

Sayous (A.-E.). - les Burses atlemantes de valeur et le commerce

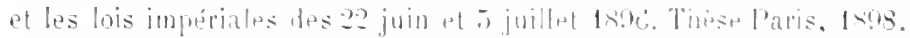

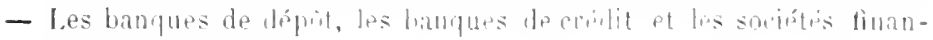
ciries. Paris, 1901.

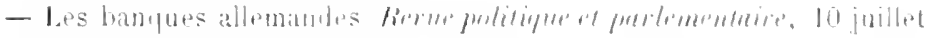
1 1.?

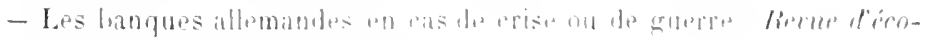

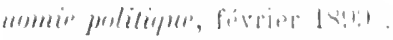

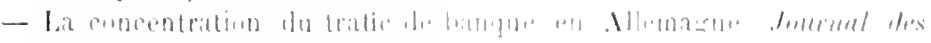

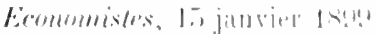

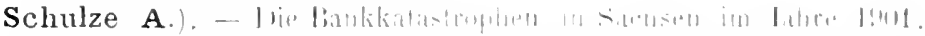

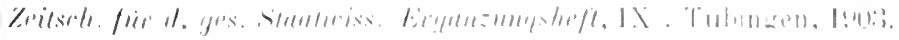

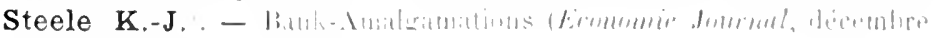
Initis.

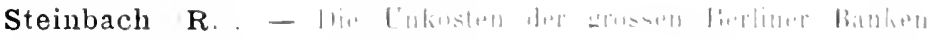

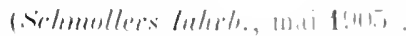

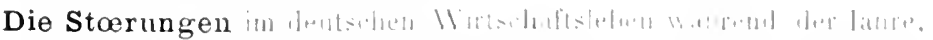

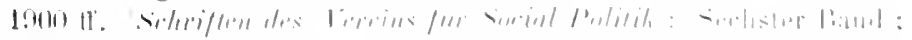

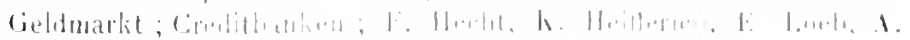

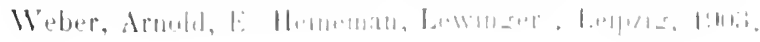


Sydow. - Ueber Gonsortien (Zeitsch. fïr das gesammle Handelsrecht t. XIX, p. 427).

Varschauer (Otto). - Physiologie der deutschen Banken. Berlin, 1903.

Weber (Adolf). - Depositenbanken und Spekulationsbanken. Leip zig, 1902 .

- Voir Störmingen...

Wermuth (A.) et Brendel (H.). - Börsengeselz (Gultentag'sche Summlung, no 41). Berlin, 1896.

Wirth (Max). - Handbuch des Bankwesens. Cologne, 1870.

\section{B. - Revues et périodiques.}

En dehors des revues et périodiques qui contiennent les articles déjà signalés, nous donnons l'énnmération des principaux journaux économiques et financiers qui tiennent d'une façon générale le public au courant des faits de concentration et qui leur ont consacré en ces dernières années un grand nombre de courts articles et de notices.

Der Deutsche OEkonomist, Berlin.

Der Berliner Actionär.

Bank Archiv. Berlin.

Die Frantifurter Zeitury.

L'Economiste Européen, Paris.

L'Economiste frumçais, Parıs.

Le marchi finantier (A. Raffalovich). Barss.

L'Information.

The Banliers Magazine, Londres.

7 he Economist.

Saling's Bürsen labrburh. Leipzig. Verlay. für Börsen nud Finanatitteratur.

Handbuch der deutschen Alitiengesellschaften (Iahrbuch der Deutschen Börsen), Leipzig. 


\section{TABLE DES MATIERES}

INTRODUCTION. - Le mouvement de concentration dans le commerce de banyue : France, Augleterre, Etats-l'nis. - Cause fórale. - Formes. - Ltude particuliere du mouvement de concentration dans les banques allemandes; division du sujet; méthode. . . . . . . . . . . . 1-23

\section{PREMIÈRE PARTIE \\ ÉTUDE SYNTHETIQUE DU MOUVEMENT DE CONCENTRATION}

CHAPITRE PREMIER. - Les banques allemandes de 1850 à 1904. - Le mouvement de concentration: tableau génèral. - Résultats densemble . . . . . . . . .

Le développement des banques allemandes (1550-1570). - Les grands établissements berlinois (1870-18\%). - La politique des grands établissements berlinois vis-i-ris des banques privées. - des banques par actions (1890-190'). - Les groupes de banques . . . . . . . . . . 25

CHAPITRE H. - Les Causes. ............ . it

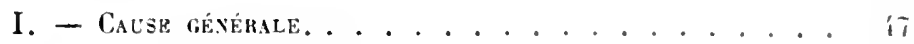

1. - La cause techmique. . . . . . . . . . . . 49

La facon mème dont les Allemands compreunent et pratiquent le commerce de banque. - Les hampues alleman. des sontà la fois des banques de dépits, des hampues de

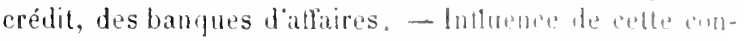
ception sur le déveloprement des hanques al le mousement de concentration . . . . . . . . . 
B. - La cause économique.

Influence du développement économique gévéral. - L'union intime des banques allemandes avec l'industrie et le commerce nationaux. - Faits et chiffres. - Les échanges d'administrateurs. - La concentration industrielle et commerciale. - Les cartells ....... .

II. - Causes particulières. . . . . . . . . . 83

A. - L'action législative. - Son etlet centralisateur . 83

$1^{\circ}$ Lois de finances. . . . . . . . . 83

20 Depotgesetz............ 87

$3^{\circ}$ Börsengesetz . . . . . . . . . 89

B. - Phénomirnes internes ì chaque établissement. . . 100

C. - La crise te 1901)-1901 . . . . . . . . 104

Ses causes. - Les grandes banques pendaut la crise. -

L'accélération du mouvement de concentration. ... 104

CHAPITRE III. - Les moyens techniques. . . . . . 116

1. Succusales. - Nombre : Caractères particuliers : $a$ ) création; b) fonctionnement. - Comparaison avec les succursates des banques anglaises et françaises. - Discontogesellschafl "l Wradentsche Bank. . . . . . .

2") Commandites. - Avantages de ce procédé d'expansiun . . . . . . . . . . . . .

Tableau des commandites des grandes banques berli. noises................. 120

$3^{\circ}$ Participations. - Avantages el modalités de ce procédé :

a) Transformation des banjues privées en sociétés par actions.............. 127

b) Acquisition ferme d'une part des actions. . . . 128

- c) Echange réciproque d'une part des actions.La Deulsche Bank et la Beryisch Marlische Bank et le Schlesischer Banlicerem. . . . . . . . .

La véritable physionomie de l'expansion des grandes banques berlinoises. - Tableaux généraux. . . . . . . 132

(l) Associations d'intérèts (futeressenyemeinschaften). Caractères particuliers . . . . . . . . . . 136 Les Consortiums de Banques. . . . . . . . 137 


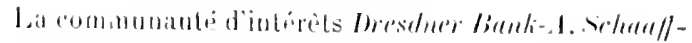

hausensirher Bantiverein . . . . . . . . 160

Influence des conditions historiques de Rillemagne sur ir choix des moyens techniques. . . . . . . . 1 hi

\section{DEUXIEME PARTIE}

\section{ETUDE ANALYTIQUE DU MOUVEMENT DE CONCENTRATION}

Genémaltís. - Divisions. . . . . . . . . . . 151

CH.MPITRE PREMHER. - La Discontogesellschaft . . . 155

io Le hévelopiemput propre de la Discontogesellschaft. -

Tableanx des bilans. - Origrine des benéfees. . . . 15i

20 La formation da groupe de la Disconto. . . . . . 16't

$3^{\circ}$ Les participalions industrielles et commerciales de la

Disconto. - Les alfaires consortiales ...... 170

CllAPJTRE II. - La Bank für Handel und Industrie . 175

1. Le développement propre de la Bank iür IIandel und Industrie. - Tableaux des bilans. - Origrine les hénéfices ................ . . 175

$2 \circ$ La formation du groupe te la Bank für Handel und Judustrie.............. 184

30 Les participations industrielles et commerciales de la Bank für tlandel und Industrie. - Les allaires consortiales................... 189

CHAPITRE III - La Deutsche Bank . . . . . . . 193

10 Le cléveloppement propre de la Deutsche Bank. - Tableaux des bilans. - Ongine des bénéfeés. . . . . 193

2n La formation du troupe de la l)eutsehe Bank. . . . . 201

3o Les particifations industrielles et enmmerciales le ta Deulsche biak. - les allidires consortiales. . . . . 20?

(:HAP'Tlil I'. - La Dresduer Bank et l'A. Schaaffhausens'cher Bankverein. . . . . . . . . . . . . ㄴ. 
I. - LE DÉvELOPPEMENT PROPRL:

A. De la Dresdner Bank . . . . . . . . 218

B. Du Schaaffhausens'cher Bkv. . . . . . . 219

Tableaux des bilans. - Origrine des bénéfices. . . . 221

II. - La formation du Grovpe Dresdner-Bank: A. Schaaffhau-

sens'cher Bkv. . . . . . . . . . . . 229

III. - Les participations industrielles et commerciales:

A. De la Dresdner Bank . . . . . . . 235

B. Du Schaaffhausens'cher Bkv . . . . . . 237

Les affaires consor tiales:

A. De la Dresdner Bank . . . . . . . . 239

B. Du Schaaffhausensch Bkv ....... 241

CONCLUSION. . . . . . . . . . . . 247

BIBLIOGRAPHIE............... 252 





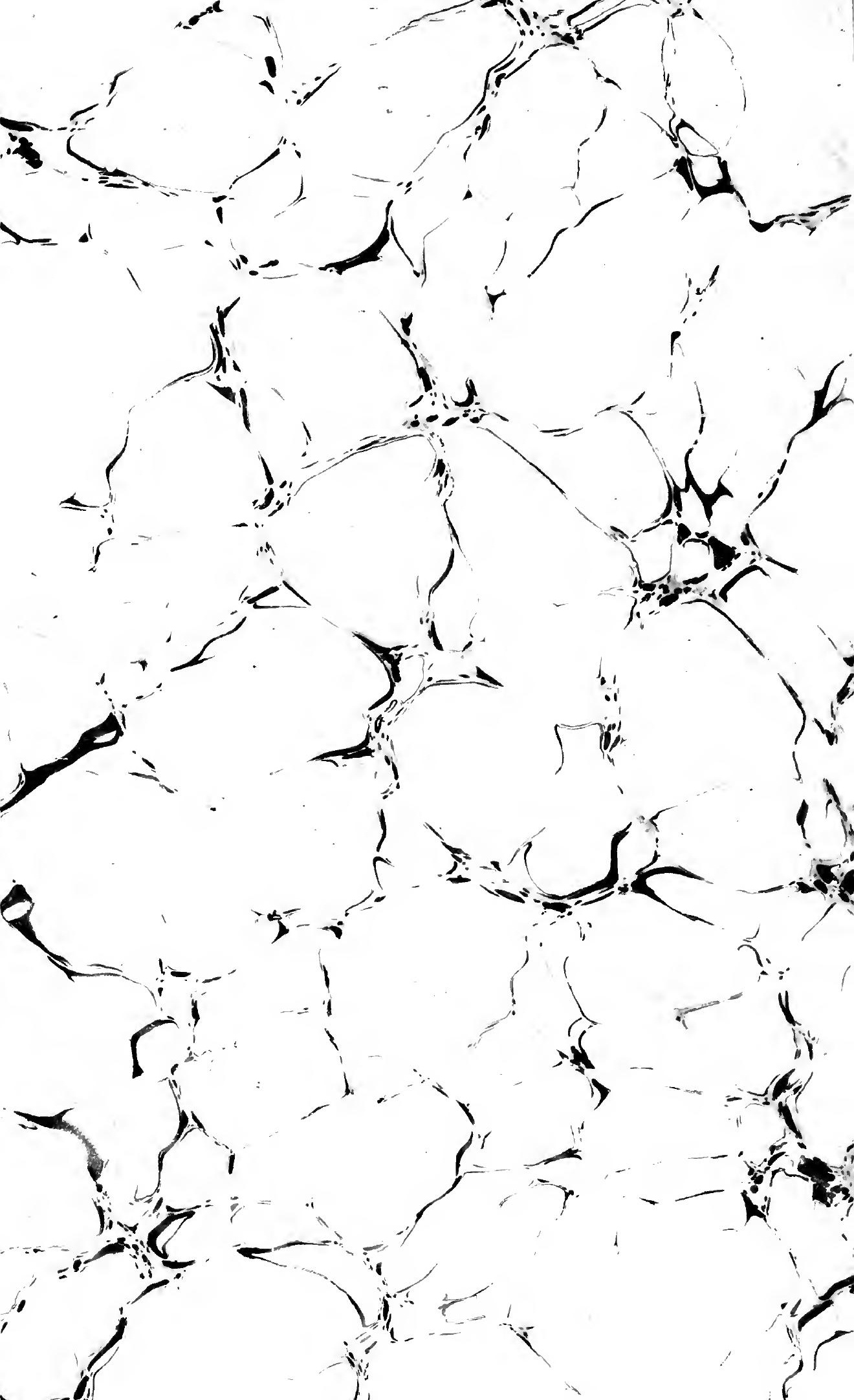




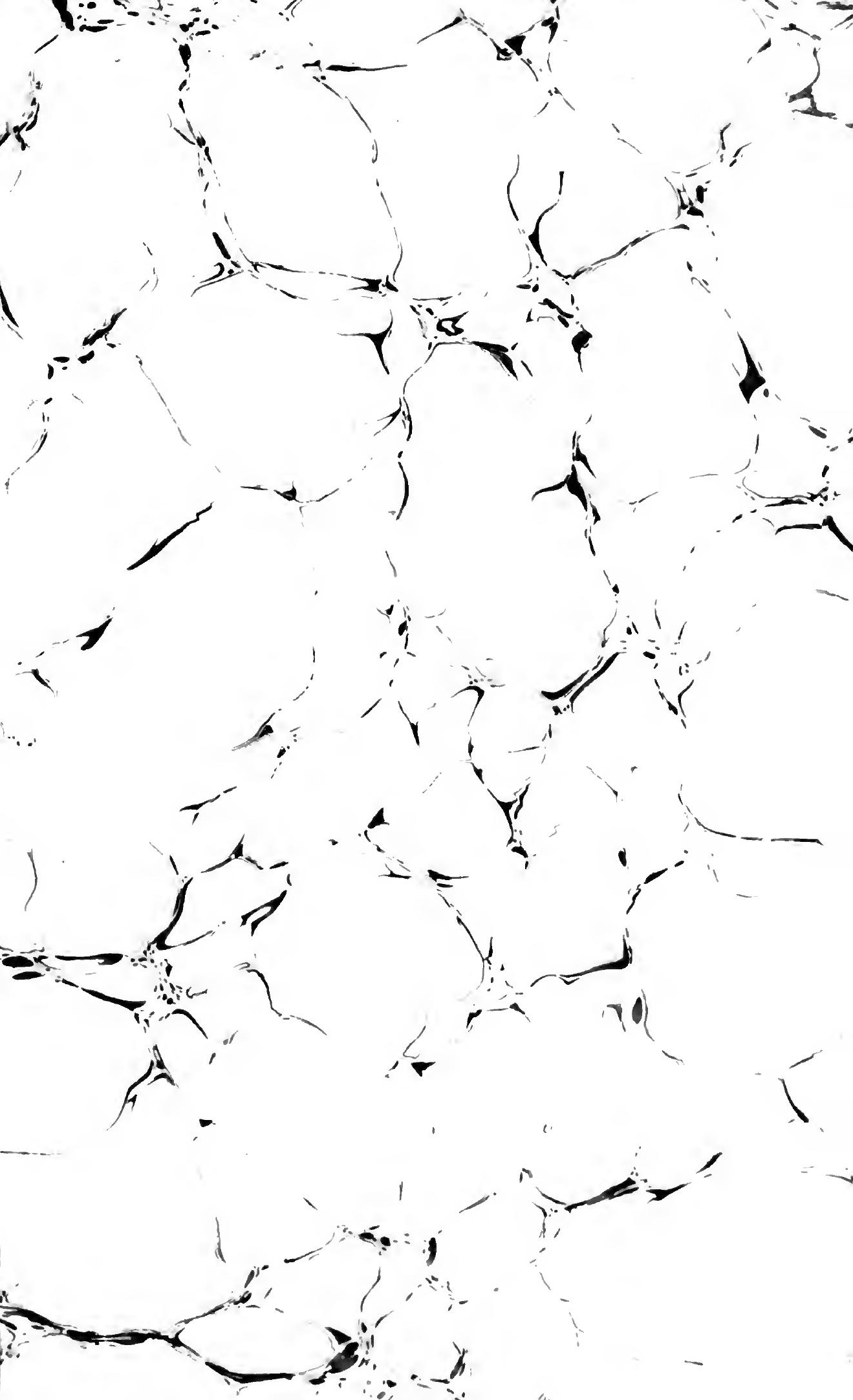


